\title{
Molecular role of PMP22 in CMT1A
}

\author{
Dissertation \\ for the award of the degree \\ “Doctor rerum naturalium" (Dr. rer. nat.) \\ of the Georg-August-Universität Göttingen \\ within in the doctoral program Biology \\ of the Georg-August University School of Science (GAUSS) \\ Submitted by \\ Doris Krauter
}

born in Vechta, Germany, 16.05.1992

Göttingen, December 2020 



\section{Examination board}

\section{Thesis committee:}

Prof. Dr. Michael W. Sereda ( $1^{\text {st }}$ Reviewer $)$

Molecular and Translational Neurology

Department of Neurogenetics

Max Planck Institute of Experimental Medicine, Göttingen

Department of Clinical Neurophysiology and Neurology

University Medical Center, Göttingen

Prof. Dr. Martin Göpfert (2 ${ }^{\text {nd }}$ Reviewer)

Department of Cellular Neurobiology

Schwann-Schleiden Research Center, Göttingen

Prof. Dr. Blanche Schwappach

Department of Molecular Biology

University Medical Center, Göttingen

Further members of the Examination Board:

Prof. Dr. Klaus-Armin Nave

Department of Neurogenetics

Max Planck Institute of Experimental Medicine, Göttingen

Prof. Dr. Dr. Hannelore Ehrenreich

Clinical Neuroscience

Max Planck Institute of Experimental Medicine, Göttingen

Prof. Dr. Christine Stadelmann-Nessler

Department of Neuropathology

University Medical Center, Göttingen

Date of the oral examination: 04.02.2021 



\section{Declaration}

I hereby declare that the PhD thesis entitled "Molecular role of PMP22 in CMT1A" has been written independently and with no other sources and aids than quoted.

Göttingen, 30.12.2020

Doris Krauter 



\section{Acknowledgements}

I want to thank Prof. Dr. Michael Sereda and Prof. Dr. Klaus-Armin Nave for welcoming me in their research group and department, respectively. Thank you for providing an independent working atmosphere and for intensive scientific discussions.

Moreover, I want to thank Prof. Dr. Martin Göpfert and Prof. Dr. Blanche Schwappach for their support as members of my thesis committee and Prof. Dr. Dr. Hannelore Ehrenreich and Prof. Dr. Christine Stadelmann-Nessler for participating as members of the examination board.

\section{Ein großes Danke an...}

... David für seine Betreuung im Labor, die zahlreichen Diskussionen und das Korrekturlesen der Thesis!

...Lisa für die schöne PhD Zeit zusammen, die wissenschaftliche Unterstützung, aber auch die schöne Zeit und die Hilfe außerhalb des Labors, ohne den Unterschlupf in deiner Wohnung wären das sehr schwierige Monate geworden!

...Stephanie and Theresa, dass ihr mir so viele Dinge im Labor beigebracht habt und natürlich auch für die schönen Momente außerhalb des Labors!

...Timon, der ein toller Lab Rotation und Master Student war und in seiner Zeit viel zum PTEN Projekt beigetragen hat!

...Sandra für die Bereitstellung und Diskussion der HNPP Daten!

...Ruth und Robert in Leipzig für anregende Diskussionen und Ideen zur Projektentwicklung!

...Beate, Christian, Michael und Lorenz für ihre exzellente technische Hilfe im Labor!

...unsere Tierpflegerinnen Tanja und Sarah, die sich hervorragend um die Mäuse kümmern!

...Michaela am MPI und Marion Kurze and der UMG für die administrative Unterstützung!

...die PhD Community der Abteilung für Neurogenetik für anregende Diskussionen, leckere Mittagessen und das ganze Drumherum!

...Ulli und Kathrin für schöne Donnerstags-Jogging-Runden vor der Arbeit!

...der ganzen Abteilung Neurogenetik für das gute Arbeitsklima und den wissenschaftlichen Austausch!

Außerdem möchte ich meinen Eltern und Geschwistern, sowie meinen Life Science Mädels Pia, Ina und Sabrina danken, die mich das ganze Studium durch begleitet und unterstützt haben. Der größte Dank gilt Arthur, du bist der beste Motivator und Unterstützer den ich mir vorstellen kann, danke! 



\section{Content}

LIST OF FIGURES I

LIST OF TABLES II

ABBREVIATIONS III

$\begin{array}{ll}\text { ABSTRACT } & 1\end{array}$

1 INTRODUCTION 2

1.1 Neuroglia in the central and peripheral nervous system 2

1.2 Schwann cell myelination 3

1.2.1 Schwann cell lineage and development 3

1.2.2 Myelinating Schwann cell architecture $\quad 4$

1.2.3 Regulation of Schwann cell myelination 6

1.3 The peripheral myelin protein of $22 \mathrm{kDa}$ (PMP22) 8

1.3.1 PMP22-associated peripheral neuropathies 9

1.3.2 Molecular function of PMP22 11

1.4 Aim of the study $\quad 12$

2 MATERIALS 14

2.1 Chemicals and Consumables $\quad 14$

$2.2 \quad$ Primer and Antibodies $\quad 16$

2.3 Plasmids and cell lines $\quad 18$

2.4 Machines and Software $\quad 19$

$\begin{array}{lll}2.5 & \text { Solutions and Buffers } & 20\end{array}$

3 METHODS 26

3.1 Animals and Behavior $\quad 26$

3.1.1 Genotyping 27

3.1.2 Rapamycin treatment 29

3.1.3 Behavior 29

3.1.4 Electrophysiology 30

3.1.5 Tissue preparation 31

$3.2 \quad$ Cell culture 31

3.2.1 Schwann cell-DRG co-culture 31

3.2.2 Primary Schwann cells 32

3.2.3 Transfection of HEK293T cells 33

3.2.4 Scratch Assay 33

3.3 Molecular biology 34

3.3.1 RNA isolation and qRT-PCR 34

3.3.2 Protein lysate extraction and quantification 35

3.3.3 Myelin purification 35

3.3.4 SDS-PAGE and Western Blot 36

$3.4 \quad$ Histology 37

3.4.1 Tissue embedding 37

3.4.2 Fluorescent staining 38

$\begin{array}{lll}\text { 3.4.3 Gallays and Blue staining } & 40\end{array}$ 
$\begin{array}{rlr}3.4 .4 & \text { Microscopy } & 40 \\ 3.5 \quad \text { Data analysis } & 41 \\ \text { 3.5.1 } & \text { Image Analysis } & 41 \\ \text { 3.5.2 } & \text { Statistical analysis } & 42\end{array}$

$4 \quad$ RESULTS $\quad 43$

4.1 ER stress as a disease mechanism in CMT1A?

4.1.1 CMT1A rats do not display ER stress. 43

4.1.2 Pharmacological prolongation of the unfolded protein response in CMT1A SC-DRG cocultures does not improve myelination in vitro.

4.2 Modulating PI3K/Akt/mTOR dependent growth signaling in PMP22 gene-dosage related

$\begin{array}{ll}\text { diseases. } & 47\end{array}$

4.2.1 PMP22 dosage perturbs the homeostasis of the major growth inhibitor PTEN in rodent disease models of CMT1A and HNPP. 47

4.2.2 Counteracting dysregulated PI3K/Akt/mTOR signaling is beneficial in an animal model of HNPP. $\quad 50$

4.2.3 Pharmacologically increasing PI3K/Akt/mTOR pathway activity in CMT1A improves myelination in vitro. 53

4.2.4 Genetic reduction of PTEN in CMT1A increases myelination in vitro and early in vivo. $\quad 54$

4.2.5 PTEN reduction in CMT1A does not alter the phenotype in aged animals. 60

4.3 Vinculin as a linker between PMP22 and growth signaling? 66

4.3.1 Vinculin localizes to focal adhesions in primary Schwanns cell in vitro and paranodal loops $\begin{array}{ll}\text { as well as Cajal bands in vivo. } & 66\end{array}$

4.3.2 CMT1A Schwann cells are less polarized and migrate slower. 67

4.3.3 CMT1A peripheral nerves display enlarged and disrupted Cajal bands. $\quad 69$

4.3.4 Vinculin depletion in Schwann cells does not lead to a PTEN-associated phenotype. $\quad 70$

4.4 Modulating Schmidt-Lanterman incisure number in CMT1A.

4.4.1 Vinculin conditional knockout mice display reduced numbers of Schmidt-Lanterman $\begin{array}{ll}\text { incisures. } & 75\end{array}$

$\begin{array}{ll}\text { 4.4.2 Depletion of Vinculin impairs the CMT1A phenotype. } & 78\end{array}$

5 DISCUSSION $\quad 83$

5.1 The primary disease mechanism of CMT1A does not involve endoplasmic reticulum stress.

5.2 Does PMP22 control the timing of myelin growth? 85

$5.3 \quad$ Molecular role of PMP22. $\quad 89$

5.4 A role for Schmidt-Lanterman incisures in sustaining Schwann cell function in CharcotMarie Tooth disease 1A. $\quad 91$

$\begin{array}{lll}5.5 & \text { Conclusions } & 94\end{array}$

6 REFERENCES $\quad 96$ 


\section{List of Figures}

Figure I: Schematic of Schwann cell development in the peripheral nervous system.............. 3

Figure II: Myelin proteins in the compact myelin sheath........................................................... 4

Figure III: The myelinating Schwann cell architecture. ............................................................. 5

Figure IV: PI3K signaling at the adaxonal and abaxonal membrane.......................................... 7

Figure V: Topology of the Peripheral myelin protein of $22 \mathrm{kDa}$ (PMP22)................................ 9

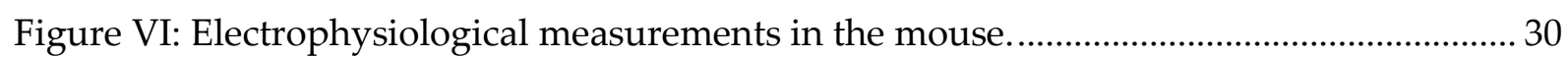

Figure VII: G-ratio analysis in whole femoral nerves using FIJI. .............................................. 41

Figure 1: ER stress markers are unaltered in CMT1A rats. ..................................................... 44

Figure 2: IFB-088 treatment in CMT1A SC-DRG co-cultures does not improve myelination. 45

Figure 3: Increase of PTEN on protein and mRNA level in a rat model of CMT1A................. 48

Figure 4: PTEN is decreased on protein and mRNA level in a mouse model of HNPP.......... 49

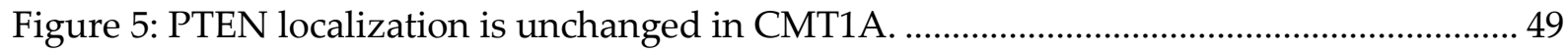

Figure 6: Inhibition of mTOR using Rapamycin decreases the number of myelin outfoldings in

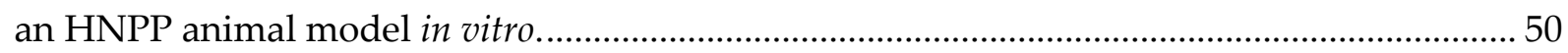

Figure 7: Inhibiting mTOR in an animal model of HNPP improves the phenotype in vivo.... 52

Figure 8: PTEN protein levels are restored in HNPP mice after Rapamycin treatment. ......... 53

Figure 9: Inhibiting PTEN increases myelination in CMT1A SC-DRG co-cultures in vitro.... 54

Figure 10: Genetic reduction of PTEN levels in CMT1A in vivo............................................... 55

Figure 11: Increased myelination in PTENhKOxCMT1A double mutants in vitro....................56

Figure 12: Increased myelination in PTENhxCMT1A double mutants at postnatal day 18.... 57

Figure 13: Internodes are slightly longer in PTENhKOxCMT1A double mutants at postnatal

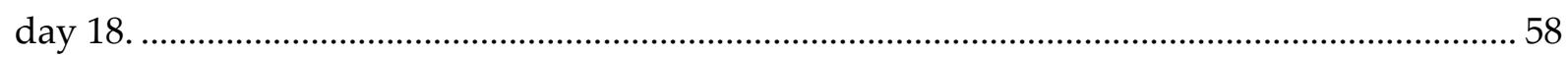

Figure 14: Full PTEN knockout in CMT1A Schwann cells deteriorates the histological phenotype.

Figure 15: Motor and sensory behavior is not altered in PTENhKOxCMT1A double mutants.

Figure 16: Electrophysiological parameters are unaltered in PTENhKOxCMT1A double mutants.

Figure 17: Histologically, PTENhKOxCMT1A double mutants do not differ from CMT1A mice at 16 weeks of age.

Figure 18: Longitudinal myelination is unaltered in PTENhKOxCMT1A double mutants at 16 weeks.

Figure 19: PTENhKOxCMT1A double mutants are unaltered at 24 weeks of age.

Figure 20: Vinculin is PMP22 dosage-dependently changed in animal models of CMT1A and HNPP.

Figure 21: Vinculin localizes to focal adhesions in vitro and bands of Cajal in the Schwann cell

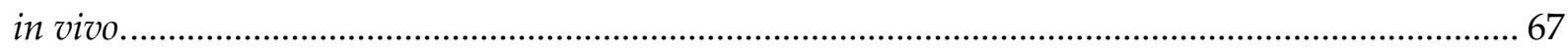

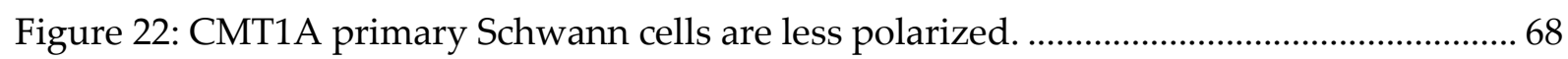

Figure 23: CMT1A primary Schwann cells migrate slower......................................................... 69 
Figure 24: CMT1A nerves display less appositions and bigger Cajal band membranes......... 70

Figure 25: Generation of Schwann cell specific Vinculin knockout mice................................. 71

Figure 26: Radial myelination is unaltered in Vinculin conditional knockout mice. ................ 72

Figure 27: Vinculin depletion does not change Cajal band and nodal integrity...................... 73

Figure 28: Vinculin expression does not influence PTEN protein level. .................................... 74

Figure 29: Teased fiber preparations of 8 weeks old VclcKO mice display reduced numbers of

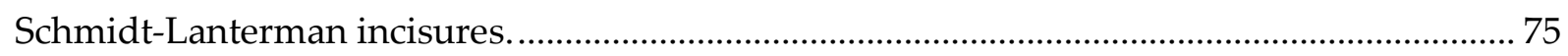

Figure 30: Teased fiber preparations of 6 days old VclcKO mice display reduced numbers of

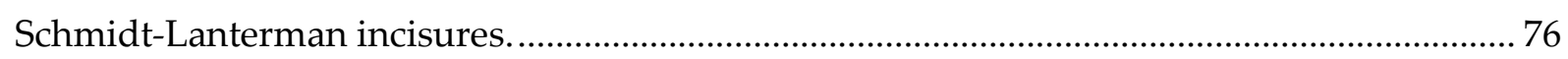

Figure 31: Teased fiber preparations of 26 weeks old VclcKO mice display reduced numbers

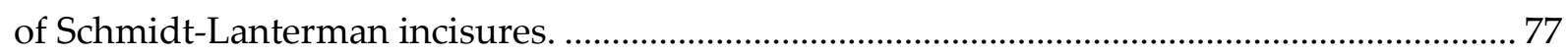

Figure 32: Vinculin depletion in CMT1A Schwann cells decreases the number of Schmidt-

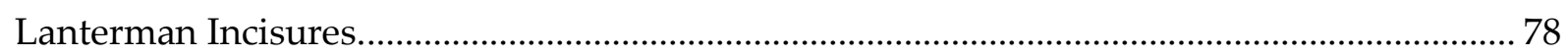

Figure 33: Vinculin depletion in CMT1A increases the number of amyelinated axons........... 80

Figure 34: VclcKOxCMT1A mice display an impaired motor behavior. ................................... 81

Figure 35: VclcKOxCMT1A double mutants show deteriorated motor electrophysiological

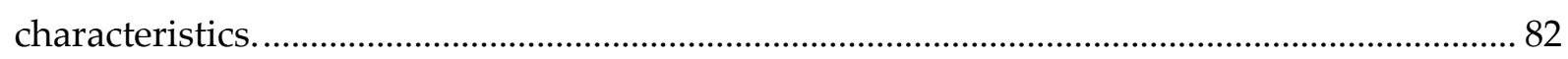

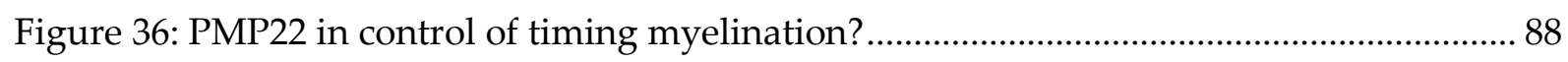

Figure 37: A role of Schmidt-Lanterman Incisures for sustaining Schwann cell function in

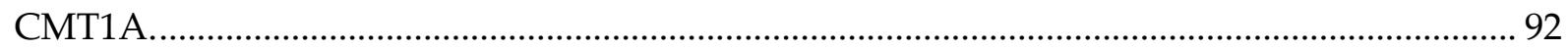

\section{List of Tables}

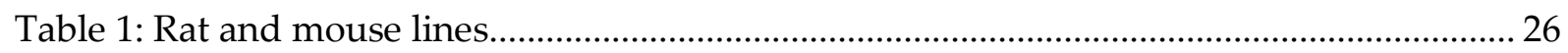

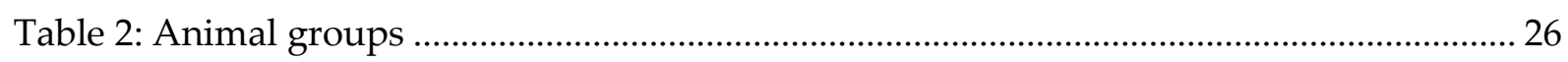




\section{Abbreviations}

ADAM22

AKT

ANOVA

ATF4/ atf4

ATF6

cDNA

$\mathrm{CHOP} /$ ddit3

CMAP

CMT

CNS

Crb3

CycloA/ cycloA

DAPI

DBcAMP

Dhh

DRG

DRP-2

Dlg1

DMSO

eIF $2 \alpha$

ER

ErbB2/ErbB3

ERK

FAK

FCS

GADD34/gadd34

GAS3

GPCR

HEK

HNPP

HMGCR/ hmgcr

HRP

HSPA5 (BiP)/ hspa5

IRE1

Lgi4

MAG

MAGI2

MAPK

MBP axonal disintegrin and metalloproteinase domain-containing protein 22

protein kinase B

analysis of variance

activating transcription factor 4

activating transcription factor 6

complementary Deoxyribonucleic acid

DNA damage-inducible transcript 3

compound muscle action potential

Charcot-Marie-Tooth

central nervous system

crumbs protein homolog 3

cyclophilin A

4', 6-Diamidin-2-phenylindol

dibutyryl cyclic adenosine monophosphate

desert hedgehog

dorsal root ganglia

dystrophin-related protein 2

discs large homolog 1

dimethyl sulfoxide

eukaryotic translation initiation factor 2

endoplasmic reticulum

epidermal growth factor receptor

extracellular signal-regulated kinases

focal adhesion kinase

fetal calf serum

growth arrest and DNA damage-inducible protein

growth arrest specific 3

G-protein coupled receptors

human embryonic kidney

hereditary neuropathy with liability to pressure palsy

(3-hydroxy-3-methyl-glutaryl-coenzyme A reductase

horseradish peroxidase

heat shock $70 \mathrm{kDa}$ protein 5

inositol requiring enzyme 1

leucine-rich glioma-inactivated 4

myelin associated glycoprotein

membrane-associated guanylate kinase inverted 2

mitogen-activated protein kinases

myelin basic protein 


\begin{tabular}{|c|c|}
\hline mRNA & messenger Ribonucleic acid \\
\hline mTOR & mammalian target of rapamycin \\
\hline $\mathrm{NaV} 1.6$ & sodium channel $\mathrm{NaV} 1.6$ \\
\hline $\mathrm{NCV}$ & nerve conduction velocity \\
\hline NDRG1 & N-myc downstream regulated gene- 1 \\
\hline NRG1 & Neuregulin 1 \\
\hline PAK1 & p21-activated kinase \\
\hline P0, MPZ & myelin protein zero \\
\hline PCR & polymerase chain reaction \\
\hline PERK & double-stranded RNA-activated protein kinase (PKR)-like ER kinase \\
\hline PFA & paraformaldehyde \\
\hline PI3K & phosphoinositide 3-kinase \\
\hline $\mathrm{PIP}_{2}, \mathrm{PI}(4,5) \mathrm{P}_{2}$ & phosphatidylinositol 4,5-bisphosphate \\
\hline $\mathrm{PIP}_{3}, \mathrm{PI}(3,4,5) \mathrm{P}_{3}$ & phosphatidylinositol 3,4,5-triphosphate \\
\hline PLC & phospholipase C \\
\hline PMP22, pmp22 & Peripheral myelin protein of $22 \mathrm{kDa}$ \\
\hline PNS & peripheral nervous system \\
\hline PTEN, pten & Phosphatase and Tensin homolog \\
\hline qRT-PCR & quantitative real-time polymerase chain reaction \\
\hline Rab35 & Ras-related protein 35 \\
\hline Rac1 & Ras-related C 3 botulinum toxin substrate 1 \\
\hline REDD1 & regulated in development and DNA damage response 1 \\
\hline RhoA & Ras-homolog family member A \\
\hline Rplp0/ rplp0 & $60 S$ acidic ribosomal protein $\mathrm{P} 0$ \\
\hline S6 & Ribosomal protein S6 \\
\hline SC & Schwann cell \\
\hline SD & standard deviation \\
\hline SDS-PAGE & sodium dodecyl sulfate polyacrylamide gel electrophoresis \\
\hline Sgk1 & serum and glucocorticoid-induced kinase 1 \\
\hline SLI & Schmidt-Lanterman incisure \\
\hline SPC & Schwann cell precursor cells \\
\hline $\mathrm{sXBP} 1 / \operatorname{sxbp} 1$ & spliced X-box binding protein 1 \\
\hline $\operatorname{tg}$ & transgenic \\
\hline TUJ1 & Neuron-specific class III beta-tubulin \\
\hline UPR & unfolded protein response \\
\hline $\mathrm{Vcl}, v \mathrm{cl}$ & Vinculin \\
\hline WT & wildtype \\
\hline YAP & yes-associated protein 1 \\
\hline
\end{tabular}





\section{Abstract}

Myelinating Schwann cells wrap around peripheral axons and allow fast neural transmission. Peripheral myelin protein of $22 \mathrm{kDa}$ (PMP22) is a transmembrane protein strongly expressed in Schwann cells. Alterations in PMP22 expression lead to motor and sensory peripheral neuropathies Charcot-Marie-Tooth disease 1A (CMT1A, PMP22 overexpression) and hereditary neuropathy with liability to pressure palsies (HNPP, PMP22 deficiency), while the molecular role of PMP22 is largely unknown. PMP22 is shuttled through the secretory pathway via the endoplasmic reticulum (ER) and the Golgi apparatus. Unaltered expression of unfolded protein response (UPR) components in CMT1A rats showed that the primary disease mechanism does not involve misfolding of PMP22 and subsequent ER stress. CMT1A is characterized by early hypermyelination of small axons and hypomyelination of big axons, whereas HNPP mice show a delayed onset of myelination and the formation of redundant myelin loops (tomacula). Previous studies from our group have partly explained the histological phenotype of CMT1A with defects in differentiation and growth regulation as one of the major growth-signaling pathways in myelination, the PI3K/AKT/mTOR pathway, is inversely regulated by PMP22 gene-dosage. Consistently, inhibition of mTOR with Rapamycin reduced tomacula formation and improved the phenotype in HNPP mice. We observed a direct correlation between PMP22 dosage and protein levels of the major inhibitor of the PI3K/AKT/mTOR pathway, Phosphatase and Tensin homolog (PTEN). In line with the notion of PTEN as the link between PMP22 and disturbed growth regulation, reducing PTEN levels either pharmacologically or genetically increased myelination in CMT1A in vitro. Genetic reduction of PTEN in CMT1A Schwann cells in vivo increased the number of myelinated axons and myelin sheath thickness early in development. However, the differentiation defect was not rescued by PTEN reduction, indicating a PTEN independent mechanism. Further, the growth defect was not ameliorated in adult animals. These results suggest a dual role for PMP22 in the timing of myelination. Therefore, PMP22 dosage is crucial in mediating the onset as well as the termination of myelination. In order to explore the functional link between PMP22 and PTEN we investigated the role of Vinculin in the Schwann cell, a potential interaction partner of PMP22 which is known to stabilize PTEN. Similarly to PTEN, Vinculin protein levels are increased in CMT1A and decreased in HNPP. Vinculin is expressed in focal adhesion plaques in primary Schwann cells in vitro and bands of Cajal in vivo. We demonstrated alterations in cell shape and migration of Vinculin overexpressing CMT1A Schwann cells and disrupted Cajal band structure in CMT1A peripheral nerves. Schwann cell specific depletion of Vinculin (VclcKO) did not alter myelin sheath thickness, behavior, electrophysiology or PTEN protein levels. Thus, PMP22 does not stabilize PTEN via Vinculin. However, VclcKO mice showed decreased numbers of Schmidt-Lanterman incisures (SLI) in peripheral nerves. SLI are cytoplasmic channels in the compact myelin internode and numbers are increased in several peripheral neuropathies. Depletion of Vinculin in CMT1A Schwann cells decreased the number of SLIs and deteriorated the phenotype, indicating a role of SLIs for sustaining Schwann cell function in CMT1A. 


\section{Introduction}

The traffic lights turn red and we immediately have our foot on the brake and react. This reaction is enabled by neurons in the central nervous system (CNS) transmitting the outside stimulus to neurons in the peripheral nervous system (PNS) and subsequently our muscles in the feet. The information is transported by electric impulses, so called action potentials, which travel from the neuronal cell body along the axon until they are converted to chemical signals in the muscle. Evolutionary considered, the faster the action potential can propagate, the better is the reaction time providing an advantage for survival.

\subsection{Neuroglia in the central and peripheral nervous system}

Evolution came up with two ways of enabling rapid action potential propagation, (i) increasing the size of the axon diameter and (ii) insulation of the axons by multiple layers of membrane, the myelin sheath (Hartline and Colman 2007, Rushton 1951). The first strategy leads to axonal gigantism, found in Bilateria such as the squid giant axon. Due to space, the concept of increasing axonal diameter is limited. The evolvement of neuroglia cells increases nerve conduction velocity 20-100 fold by insulation of the axon with multiple membrane layers, the so-called myelin sheath. Thereby, action potential propagation is restricted to the nodes of Ranvier, small non-myelinated regions with clustered Sodium channels, resulting in the faster saltatory nerve conduction while saving space.

Neuroglial cells differ between the CNS and the PNS. Astrocytes, microglia and oligodendrocytes are the glia cells in the CNS. Astrocytes are the most abundant ones and are important in storage and supply of nutrients to the axons, in the formation of the blood-brain barrier and neurotransmitter clearance from the synaptic cleft (Volterra and Meldolesi 2005, Giaume et al 2010). Microglia can sense pathogens in the CNS and subsequently transform in an activated state. This allows microglia to fulfill repair functions after injury such as phagocytosis of debris (Lloyd and Miron 2019, Butovsky and Weiner 2018). Oligodendrocytes are the myelin-forming cells in the CNS, while one oligodendrocyte wraps around multiple axons to form adhesive membrane layers, the myelin sheath. Moreover, oligodendrocytes are involved in the metabolic support of the axons (Funfschilling et al 2012, Nave 2010). In the PNS, myelin is formed by Schwann cells. In contrast to oligodendrocytes, one Schwann cell forms myelin around a single axon (1:1 ratio). Furthermore, Schwann cells provide trophic support for the axon, have immunomodulatory functions and dedifferentiate during injury to facilitate remyelination (Armati et al 1990, Armati and Mathey 2013, Jessen and Mirsky 2016). This thesis focuses on the myelination in the PNS, thus, the development and regulation of Schwann cell myelination is further introduced in the following section. 


\subsection{Schwann cell myelination}

\subsubsection{Schwann cell lineage and development}

Early in embryonic development Schwann cells originate from the neural crest and migrate to the developing peripheral nerves to form Schwann cell precursor cells (SPC), which are in contact with multiple axons. Further, SPC develop into immature Schwann cells around bundles of axons before a process called radial sorting starts. Immature Schwann cells develop in parallel with the basal lamina, the endoneurium and blood vessels (Jessen and Mirsky 2005). During radial sorting, axons larger than $1 \mu \mathrm{m}$ are sorted into a 1:1 relationship with Schwann cells (pro-myelinating Schwann cell), whereas smaller axons are grouped in so-called Remak bundles, enwrapped by a single membrane layer of a non-myelinating Schwann cell (Jessen and Mirsky 2005, Lobsiger et al 2002, Sherman and Brophy 2005). Figure I displays a schematic of Schwann cell development.

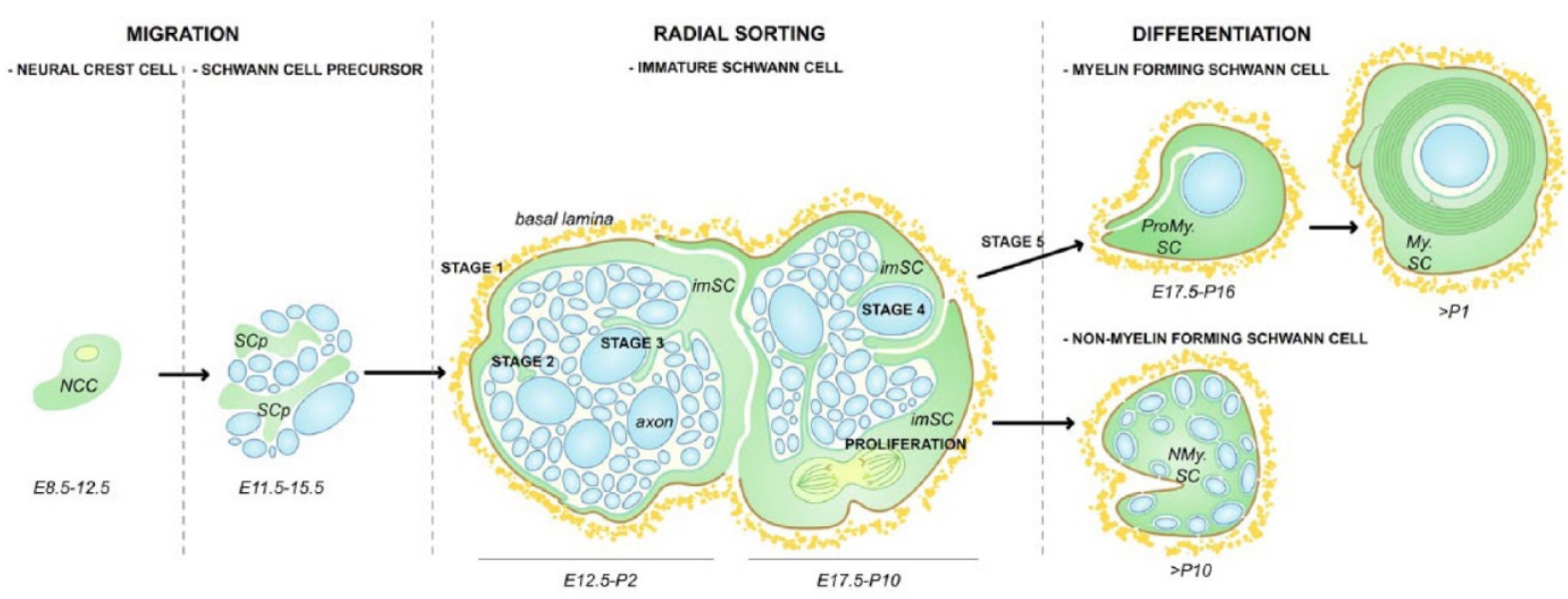

Figure I: Schematic of Schwann cell development in the peripheral nervous system.

Neural crest cells migrate to developing axons and form Schwann cell precursor cells. These further develop in immature Schwann cells, which perform the radial sorting process by production of the basal lamina (stage 1), before Schwann cell precursors (stage 2) recognize axons larger $1 \mu \mathrm{m}$ (stage 3) and form pro-myelinating Schwann cells. Pro-myelinating Schwann cells and large axons are sorted in a 1:1 ratio while small axons are grouped in Remak bundles by non-myelinating Schwann cells. Figure adapted from Feltri et al 2016.

The molecular mechanisms underlying Schwann cell development are a complex interplay between signals from the axon, the extracellular matrix and intrinsic Schwann cell pathways. A main regulator of various Schwann cell functions is Neuregulin-1 (NRG1) (Birchmeier and Nave 2008). On the one hand, the amount of NRG1 type III on the axonal surface and its interaction with epidermal growth factor receptors ErbB2/3 as well as axonal disintegrin and metalloproteinase domain-containing protein 22 (Adam22) interacting with Schwann cell secreted leucine-rich glioma-inactivated 4 (Lgi4) have shown to be important in radial sorting and recognition of axon size by Schwann cells (Birchmeier and Nave 2008, Feltri et al 2016). On the other hand, extracellular matrix molecules such as laminin and collagen and their receptors integrin, G-protein coupled receptors (GPCR) and focal adhesion kinase (FAK) mediate cytoskeletal 
changes in order to sort axons (Feltri et al 2002, Yu et al 2005, Monk et al 2011, Grove et al 2007).

Further details on myelination and the associated signaling pathways are introduced in section 1.2.3, after describing the complex myelinating Schwann cell architecture, which has to be kept in mind to understand the simultaneously occurring longitudinal and radial myelin growth.

\subsubsection{Myelinating Schwann cell architecture}

Myelin sheaths are multiple layers of Schwann cell plasma membrane wrapped around an axonal segment. Longitudinally, the Schwann cell-axon unit can be divided in the internode, juxtaparanode, paranode and node of Ranvier. Internodes consist of compact myelin, reach up to $2 \mathrm{~mm}$ length (Hildebrand et al 1994) and insulate the axons to allow saltatory action potential propagation. The adhesive connection between the plasma membrane layers is established by myelin proteins. The transmembrane protein myelin protein zero (MPZ, P0) is the most abundant protein in the PNS myelin ( $45 \%$, Siems et al 2020). P0 interacts with itself on the extracellular site of the plasma membrane forming the intraperiod line (Shapiro et al 1996). In the cytoplasm, P0 interacts with myelin basic protein (MBP) to form the major dense line (Martini et al 1995). Another protein of the compact myelin is the peripheral myelin protein of $22 \mathrm{kDa}$ (PMP22), a transmembrane protein interacting with P0 (D'Urso et al 1999). Figure II
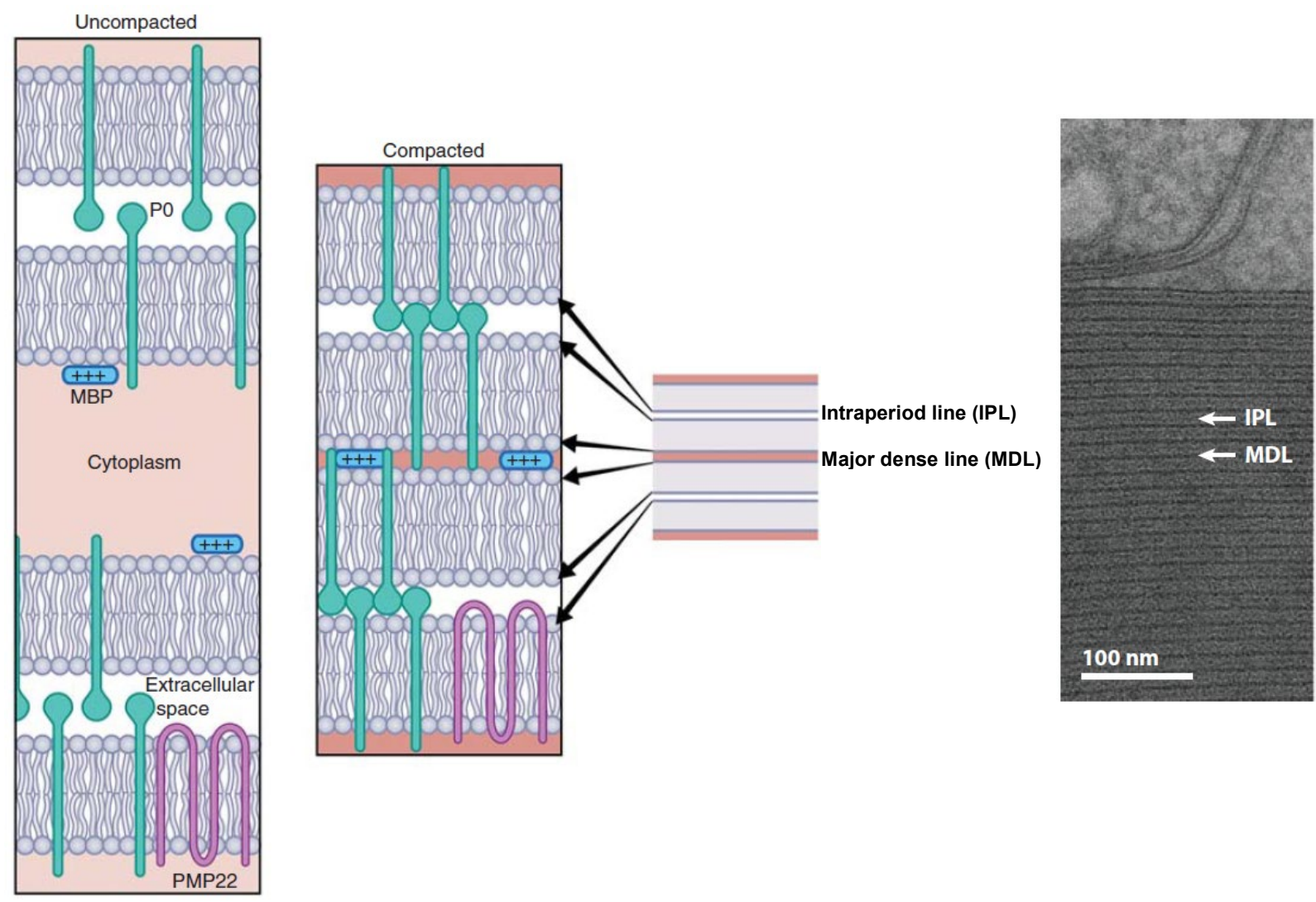

Figure II: Myelin proteins in the compact myelin sheath.

Schematic image on the left displays localization of myelin proteins P0, MBP and PMP22 in the PNS and their interaction results in the compaction of the myelin and formation of the intraperiod and major dense line Adapted from Salzer 2015. Electron micrograph image on the right shows the compaction and formation of intraperiod line (IPL) and major dense line (MDL) in a cross-section of a peripheral nerve. Adapted from Nave and Werner 2014. 
shows the compaction of the myelin sheath by the aforementioned myelin proteins $\mathrm{P} 0, \mathrm{MBP}$ and PMP22.

The longitudinal borders of the myelin sheath are the paranodal loops, cytoplasm filled membrane layers connected to the juxtaparanodal region of the axon by specialized adhesion molecules, and microvilli in the outermost layer, adjacent to the node of Ranvier (Rasband and Peles 2021, Salzer 2015). The inner cytoplasmic collar (inner mesaxon) forms the adaxonal noncompart compartment of the Schwann cell which is enriched in adhesion molecules connecting the innermost layer of the Schwann cell with the axon and processing signaling cues from the axon (Poliak and Peles 2003). The abaxonal site of the Schwann cell compromises of the Schwann cell nucleus and the bands of Cajal, longitudinal cytoplasmic channels interrupted by circular appositions, which tightly connect the abaxonal membrane with the underlying compact myelin sheath by a dystroglycan-Drp2-periaxin complex (Sherman et al 2012). Moreover, non-compact cytoplasmic channels also exist in the compact internode. The socalled Schmidt-Lanterman incisures (SLI) are funnel-shaped cytoplasm-rich structures, containing a single microtubule and facilitate a radial connection between the ab-and adaxonal compartment of the Schwann cell. The membrane layers are connected by gap junctions and autotypic adherence junctions, similarly observed in the paranodal loops. The whole Schwann cell-axon unit is covered by a basal lamina, which on the one hand provides mechanical

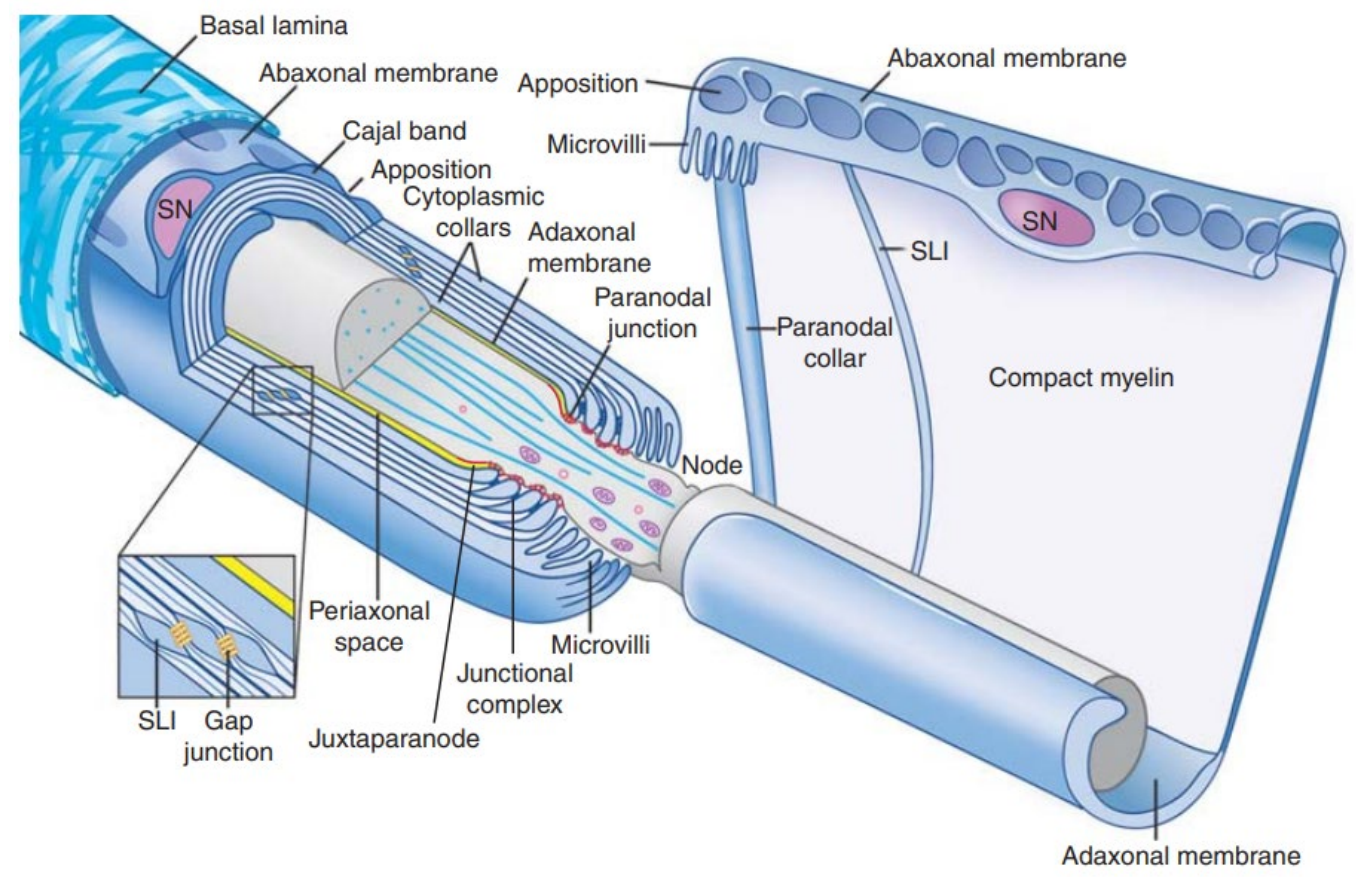

Figure III: The myelinating Schwann cell architecture.

The scheme shows a myelinated internode, covered by the basal lamina on the abaxonal site which consists of cytoplasmic Cajal bands and appositions connecting the outermost SC membrane to the compact myelin. SchmidtLanterman incisures (SLI) are cytoplasmic channels radially connected by gap junctions in the compact myelin sheath. The adaxonal membrane connects to the axon by paranodal loops and microvilli in the area of the node of Ranvier. On the right an unrolled Schwann cell is displayed. Adapted from Salzer 2015. 
stability and on the other hand facilitates signaling from the extracellular matrix via laminins and integrins (Court et al 2006, Feltri et al 2016).

All non-compact myelin structures are enriched in cytoskeletal proteins such as f-actin, supporting the assumption of their role in enabling longitudinal and radial transport along the axon. This is supported by the view of a myelinating Schwann cell being highly polarized, longitudinally from node to node and radially from the abaxonal to the adaxonal membrane (Pereira et al 2012), providing sites of localized signaling (Heller et al 2014). Figure III displays the architecture of a myelinating Schwann cell.

\subsubsection{Regulation of Schwann cell myelination}

Schwann cells start to myelinate the axon around birth and continue throughout adulthood. Myelin sheath thickness and internodal length are proportional to the axon diameter. Schwann cells have to orchestrate not only radial but also longitudinal myelin growth simultaneously. In order to produce the high amount of membrane needed for myelination, Schwann cells strongly upregulate lipid and myelin protein expression during myelination.

A master regulator of growth signaling in the peripheral nervous system is Neuregulin 1 (NRG1). On the axonal site, Neuregulin 1 type III initiates myelination and determines myelin sheath thickness via the activation of ErbB2-ErbB3 receptors on the Schwann cell (Michailov et al 2004, Taveggia et al 2005, Brinkmann et al 2008). Subsequently, the (i) PI3K/AKT/mTOR, (ii) phospholipase $C-\gamma$ (PLC) and (iii) the mitogen-activated protein kinases (MAPK)/ extracellular signal-regulated kinases (ERK) pathway are activated, leading to increased expression of myelin proteins and lipid genes in the Schwann cell (Newbern and Birchmeier 2010). Moreover, Schwann cell myelination is regulated by extrinsic signals from the extracellular matrix. Laminin and collagen from the basal lamina interact with integrin and dystroglycan receptors on the abaxonal membrane further influencing Ras-homolog family member A (RhoA) and Rasrelated C3 botulinum toxin substrate 1 (Rac1) activity, which are crucial for f-actin organization in the Schwann cell (Nodari et al 2007, Benninger et al 2007). Recent studies have shown that mechanical cues from the extracellular site drive longitudinal myelination via the activation of yes-associated protein 1 (YAP) and termination of myelin elongation by the polarity protein crumbs protein homolog 3 (Crb3) and the HIPPO pathway (Poitelon et al 2016, Fernando et al 2016, Tricaud 2017). Taken together, the complex interplay of axonal, extracellular and Schwann cell intrinsic signaling pathways is needed to ensure proper myelination of axons radially and longitudinally. In this work, we have focused on the PI3K/AKT/mTOR signaling pathway and its major inhibitor PTEN, further introduced in the following parts.

\section{PI3K/AKT/mTOR signaling pathway}

Phosphoinositide 3-kinases (PI3K) catalyzes the conversion of small lipids Phosphatidylinositol 4,5bisphosphate ( $\mathrm{PIP}_{2}$ ) to Phosphatidylinositol 3,4,5-triphosphate ( $\left.\mathrm{PIP}_{3}\right)$ at the plasma membrane, which subsequently activates protein kinase $B$ (AKT) and downstream mammalian target of Rapamycin (mTOR). Studies have shown, that alterations in the PI3K/AKT/mTOR signaling pathway strongly influence myelin sheath thickness in the PNS. Increasing pathway activity 
by AKT activation results in hypermyelination of axons and increased myelin growth, whereas AKT and mTOR depletion lead to hypomyelination and reduced myelin growth (DomenechEstevez et al 2016, Norrmen et al 2014). Thereby, the correct timing and activating status seems to be critical to mediate the switch from promyelinating Schwann cells to myelinating Schwann cells, as increased mTOR signaling delays the onset of myelination early in development but increases myelination in adulthood (Figlia et al 2017, Figlia et al 2018). Recent studies have shown that PI3K signaling is not only mediated by NRG1 from the axonal site but also on the abaxonal site in the bands of Cajal by laminin/integrin dependent pathways (Heller et al 2014). The authors showed a distinct regulation of myelination by PI3K on the abaxonal site, which is mediated through serum and glucocorticoid-induced kinase 1 (Sgk1) and $N$-myc downstream regulated gene-1 (NDRG1). Of high interest are not only the factors which drive, but also terminate myelination to avoid hypermyelination, a common feature in some peripheral neuropathies (Adlkofer et al 1997, Bolino et al 2004). Expression levels of AKT, ERbB2 and mTOR are downregulated after myelination is complete (Heller et al 2014, Sheean et al 2014), consistent with data that increasing AKT activation in adults drives hypermyelination (Figlia et al 2017). Regulated in development and DNA damage response 1 (REDD1) and Ras-related protein (Rab35) control myelination via mTOR, as depletion of these proteins leads to an increase in mTOR activity and hypermyelination (Noseda et al 2013, Sawade et al 2020), whereas inhibition of mTOR by Rapamycin administration was shown to decrease hypermyelination in Rab35 deficient mice (Sawade et al 2020). Figure IV displays two sites of PI3K signaling in the Schwann cell, adaxonally mediated by NRG1 early in development and abaxonally regulated by laminin and integrin from the basal lamina (Heller

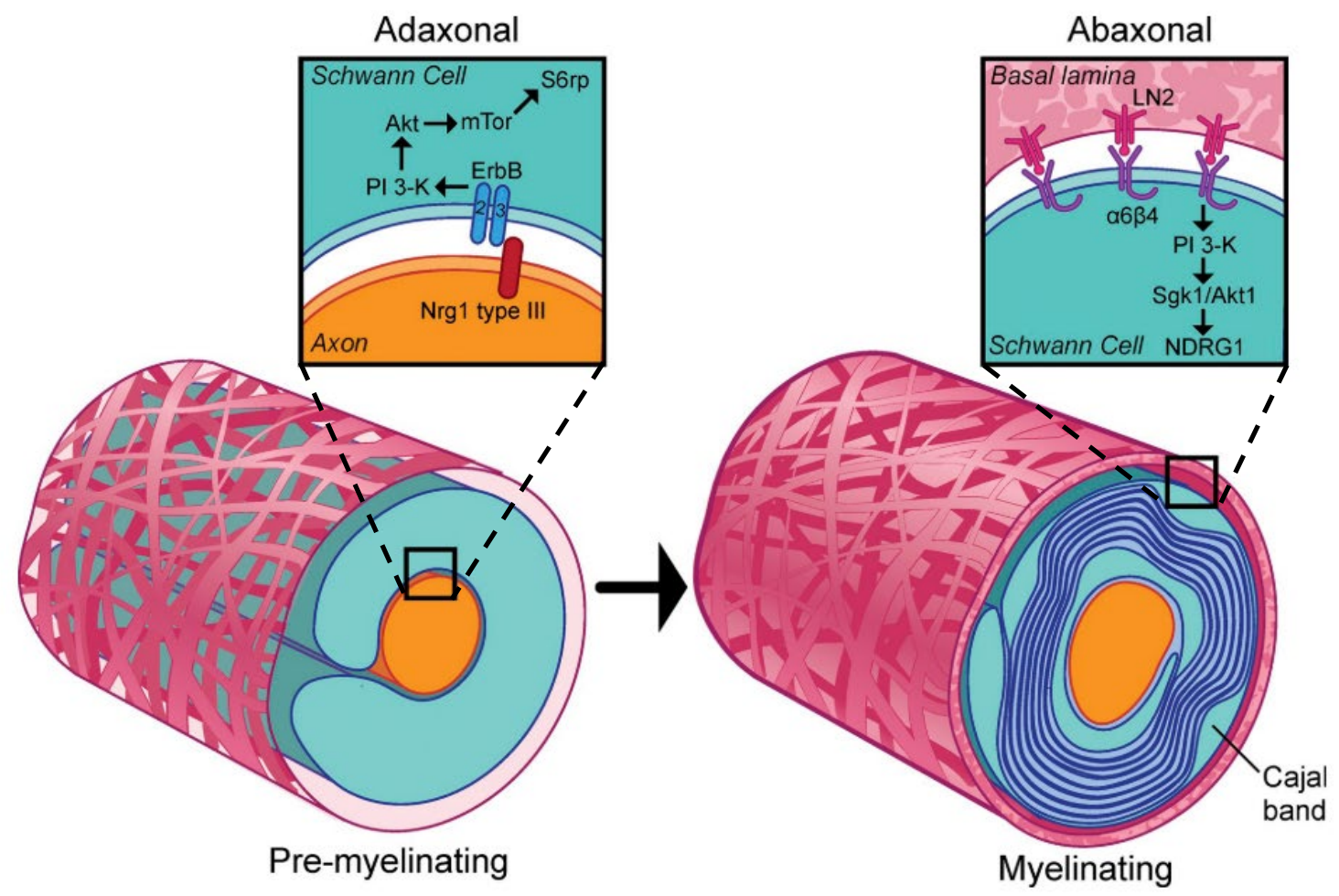

Figure IV: PI3K signaling at the adaxonal and abaxonal membrane.

PI3K/AKT signaling regulates myelin growth in early development at the adaxonal site (left scheme) and restricts myelin growth via the abaxonal compartments in later stages. Taken from Heller et al 2014. 
et al 2014). The complex interplay of timely and spatially regulation of PI3K/AKT/mTOR signaling is needed to facilitate radial and longitudinal myelination in the Schwann cell.

\section{PTEN}

Phosphatase and tensin homolog (PTEN) is best known for its lipid phosphatase activity in antagonizing PI3K by dephosphorylating $\mathrm{PIP}_{3}$ to $\mathrm{PIP}_{2}$ and subsequently inhibition of $\mathrm{AKT} / \mathrm{mTOR}$ (Lee et al 1999). PTEN is a tumor suppressor gene, heavily investigated for its role in various forms of cancer as it is often found to be mutated or deleted in tumors (Steck et al 1997, Liaw et al 1997). Thereby, the PTEN activity is not only mediated by expression levels but also subcellular localization in the cell and interaction with other proteins (Lee et al 2018b). Next to the role as a lipid phosphatase, PTEN has been described as a protein phosphatase, dephosphorylating itself and other proteins, for instance FAK (Tamura et al 1998). Interaction of PTEN with membrane-associated guanylate kinase inverted 2 (MAGI2) and actin-binding protein Vinculin prevents PTEN from degradation and directs the protein to specific membrane sites (Subauste et al 2005). This allows the controlling of $\mathrm{PIP}_{3}$ levels at the plasma membrane and contributes to the polarization and subsequently migration and motility of the cell, partly independent of PI3K/AKT signaling (Song et al 2012).

Establishing a $\mathrm{PIP}_{2}-\mathrm{PIP}_{3}$ gradient in the Schwann cell cytoplasm lead to polarization of the cell and regulates cytoskeletal dynamics, needed for myelin sheath wrapping and the termination of myelination (Pereira et al 2012). PTEN depletion in Schwann cells increases AKT/mTOR activity and leads to hypermyelination of axons in areas of non-compact myelin such as paranodal loops and SLIs, due to sustained AKT activation (Goebbels et al 2012, Figlia et al 2017). One mechanism of terminating myelination is the interaction of discs large homolog 1 (Dlg1) with PTEN at the adaxonal membrane resulting in a downregulation of the PI3K/AKT signaling, whereas depletion of Dlg1 in Schwann cells leads to a transient hypermyelination of axons (Cotter et al 2010, Macklin 2010).

\subsection{The peripheral myelin protein of $22 \mathrm{kDa}$ (PMP22)}

PMP22 is a transmembrane protein consisting of four transmembrane domains with a glycosylation at one of the two extracellular loops and N- and C-termini facing intracellularly (Figure V). The abundance of PMP22 is $2-5 \%$ of the total myelin proteins (Pareek et al 1993). First, PMP22 transcript upregulation was observed in serum-depleted fibroblasts and the transcript was named growth arrest specific 3 (GAS3) (Manfioletti et al 1990). Independently, PMP22 mRNA was shown to be decreased after nerve injury (Spreyer et al 1991, Welcher et al 1991) and increased during myelination (Snipes et al 1992). Thereby, PMP22 is expressed in various tissues throughout the body, while expression levels are strongly increased in Schwann cells (Suter et al 1994). PMP22 is associated with a variety of peripheral neuropathies (section 1.3.1), increasing the interest in understanding the molecular role of PMP22 in health and disease (section 1.3.2). 


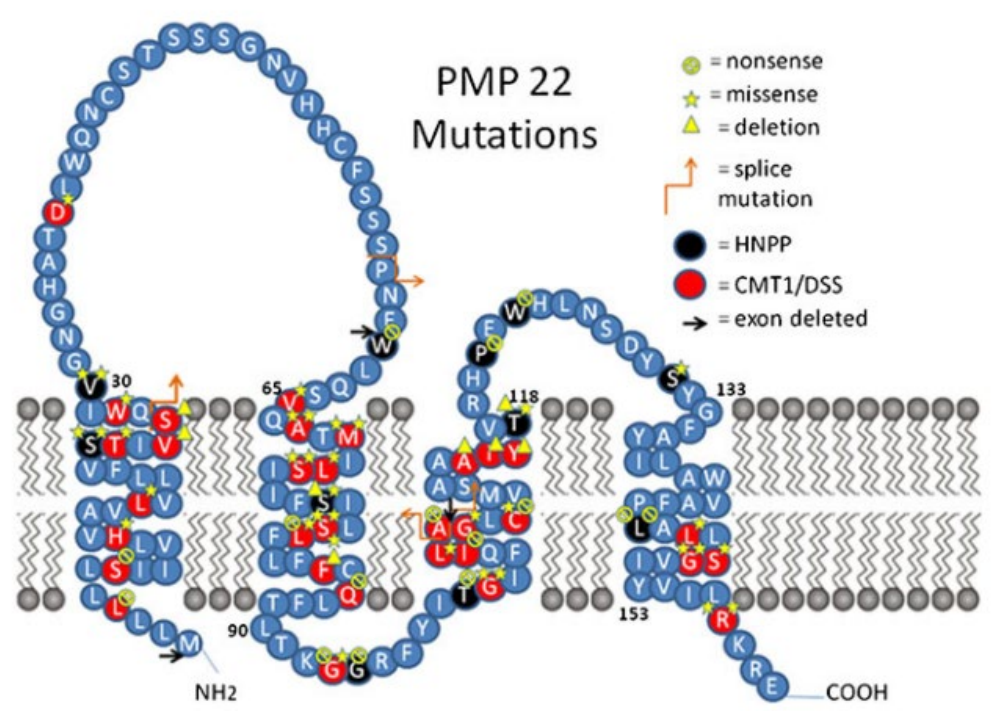

Figure V: Topology of the Peripheral myelin protein of $22 \mathrm{kDa}$ (PMP22).

PMP22 consists of four transmembrane domains, linked by two extracellular loops, while N- and C-terminus face intracellularly. Mutations causing CMT1E forms are indicated. Figure adapted from Li et al 2013.

\subsubsection{PMP22-associated peripheral neuropathies}

Autosomal dominant inherited neuropathies are classified in demyelinating Charcot-MarieTooth type 1 (CMT1, previous HMSN-1) and axonal CMT2 (HMSN-2) (Dyck and Lambert 1968) CMT has a prevalence of approximately 1:5000 and more than half of the cases are caused by alterations related to the PMP22 gene (Martyn and Hughes 1997). PMP22 is genetically encoded on chromosome 17p11.2-p12, a mutation hotspot area (Boerkoel et al 1999). Point mutations as well as changes in PMP22 gene-dosage lead to different kinds of peripheral neuropathies. Point mutations in PMP22 are very rare and can display mild as well as very severe forms of neuropathy (CMT1E). A heterozygous deletion of chromosome 17p11.2-p12 is the genetic basis of hereditary neuropathy with liability to pressure palsies (HNPP), due to a deficiency in PMP22 and a duplication in the same region leads to Charcot-Marie-disease type 1A (CMT1A) by PMP22 overexpression.

\section{Charcot-Marie-Tooth disease type 1A (CMT1A)}

CMT1A is caused by an overexpression of PMP22 (Lupski 1992, Timmerman et al 1990). The clinical phenotype manifests in the first two decades of life, characterized by foot hand deformities accompanied with sensory and motor deficits while disease severity can vary (Pareyson and Marchesi 2009). Patients display a uniform slowing of nerve conduction velocity and reduced amplitudes (Thomas et al 1997, Lewis et al 2000). On the histological level, increased formation on onion bulbs, shortened internodes, thinner myelin sheaths and axonal loss are observed (Gabreels-Festen et al 1995).

Different rodent animal models are used to study CMT1A pathomechanisms in vivo (Fledrich et al 2012b, Sereda and Nave 2006). The CMT1A rat model carries three copies of the mouse Pmp22 gene and closely resembles the human phenotype (Sereda et al 1996, Niemann et al 1999). CMT1A rats display early hypermyelination of small caliber axons and later 
demyelination, onion-bulbs, decreased axonal diameter and axonal loss, while grip strength and electrophysiological parameters are deteriorated. Moreover, CMT1A rats show a high disease variability, which is also observed in patients, even monozygotic twins (Fledrich et al 2012a, Garcia et al 1995). Several mouse models exists for CMT1A. Seven PMP22 copies are carried by the $\mathrm{C} 22$ mouse model. This mouse line strongly overexpresses PMP22 and displays a severe phenotype with a reduced life span (Huxley et al 1996). C22 mice are shown to form aggregates and display impairment in protein degradation systems (Fortun et al 2006). Thus, C22 might better serve as a model for severe CMT forms such as Déjerine Sòttas syndrome than the more mildly CMT1A. Other models, carrying four copies of the human PMP22 gene, the C61 and C3 mouse line, are mildly affected compared to the CMT1A rat, but provide sufficient models to study PMP22 gain of function mechanisms in vivo (Huxley et al 1998, Verhamme et al 2011).

\section{Hereditary neuropathy with liability to pressure palsies (HNPP)}

A deletion on chromosome 17p11.2-p12 results in PMP22 deficiency and causes hereditary neuropathy with liability to pressure palsies (HNPP). The clinical symptoms of HNPP patients are mild (Kumar et al 2002). As the name indicates, upon mechanical stress such as pressure or compression patients show palsies and paresthesias (Mouton et al 1999). With disease progression, older patients display sensory loss, muscle atrophy and weakness in the extremities. The electrophysiological pattern in HNPP patients is not as clearly defined as in CMT1A. Slowed nerve conduction velocity can be observed at sites susceptible for compression while other regions are unaffected ( $\mathrm{Li}$ et al 2002). A hallmark of the HNPP disease is the formation of tomacula, extensive formation of myelin sheaths at cytoplasmic areas, such as paranodes and Schmidt-Lanterman incisures leading to deformed and constricted axons and subsequently demyelination (Mandich et al 1995, Li et al 2013).

Mice with a heterozygous deletion in PMP22 serve as a rodent model for the HNPP disease (Adlkofer et al 1997). Similar to patients, the mice develop tomacula and demyelination during disease progression with minimal slowing of nerve conduction velocity. Upon mechanical stress, conduction block is induced in HNPP mice and they recover slower than their respective wildtype controls (Bai et al 2010).

\section{PMP22 point mutations}

PMP22 point mutations are classified as the CMT1E subgroup, making up 1-5 \% of all CMT1 cases (Bird 1993). Thereby, the phenotype changes from mild sensitive neuropathies to severe demyelinating neuropathies (Russo et al 2011). Best studied are the Trembler and TremblerJ mouse models, carrying PMP22 point mutations in the first and fourth transmembrane domain, respectively (Suter et al 1992b, Suter et al 1992a). Both models display abnormally increased Schwann cell proliferation and demyelination, while the Trembler-J phenotype is more severe. PMP22 passes the secretory pathway via endoplasmic reticulum and Golgi apparatus before transport to the plasma membrane (Pareek et al 1997). Point mutations in PMP22 are associated with retaining protein in the ER and an upregulation of the endosomal 
and lysosomal degradation pathway as well as the unfolded protein response (UPR) (Ryan et al 2002, Fontanini et al 2005, Notterpek et al 1997). Thereby, activation of autophagy improves myelination in SC-DRG co-cultures of Trembler-J mice in vitro (Rangaraju et al 2010). Moreover, Trembler-J mice display an upregulation of the unfolded protein response (UPR) due to retained protein in the ER and alleviating stress by curcumin improves the phenotype (Okamoto et al 2013). In this study we addressed whether an activation of the unfolded protein response is a potential disease mechanism not only in PMP22 point mutations but also PMP22 overexpressing CMT1A. Therefore, the molecular mechanisms in the ER stress response are further introduced.

\section{The unfolded protein response (UPR)}

Myelin proteins such as PMP22 and P0 are strongly upregulated during myelination. Therefore, high amounts of proteins have to be translated in the ER. Point mutations and overexpression can lead to misfolding of proteins and accumulation in the ER, so-called ER stress (D'Urso et al 1998, Notterpek et al 1999, Ryan et al 2002). Hence, the unfolded protein response (UPR) is activated to reduce the protein folding load in the ER, while persistent activation leads to cell death (Walter and Ron 2011). Protein folding conditions in the ER are recognized by three different transmembrane signaling molecules in the ER (i) inositol requiring enzyme 1 (IRE1), (ii) double-stranded RNA-activated protein kinase (PKR)-like ER kinase (PERK) and (iii) activating transcription factor 6 (ATF6), which downstream activate the transcription of chaperones and protein degradation systems (Ron and Walter 2007, Harding et al 2000). Upon ER stress, PERK phosphorylates itself and downstream leads to the phosphorylation of eukaryotic translation initiation factor 2 (eIF2 $\alpha$ ) (Harding et al 1999, Scheuner et al 2001). This results in the attenuation of the overall mRNA translation in the cell, while the translation of the activating transcription factor 4 (ATF4) is specifically enhanced. One important target of ATF4 is growth arrest and DNA damage inducible 34 (GADD34), which acts as a negative feedback mechanism by dephosphorylating eIF2 $\alpha$ to reactivate translation (Novoa et al 2001, Connor et al 2001). Specific inhibition of GADD34 prolongs the attenuation of translation in cells with high ER stress, which proved to be a beneficial treatment in an animal model of P0 point mutation (CMT1B, Das et al 2015) and opens up new therapeutical target in peripheral neuropathies.

\subsubsection{Molecular function of PMP22}

Especially in cell culture analysis, PMP22 was shown to regulate cell proliferation, differentiation and apoptosis (Fabbretti et al 1995, Brancolini et al 1999, Brancolini et al 2000, Sancho et al 2001). Moreover, PMP22 is involved in regulating actin-mediated function such as cell migration and cell morphology (Lee et al 2014, Nobbio et al 2004, Brancolini et al 1999). As previously described, PMP22 is widely expressed throughout the body. In epithelial cells, PMP22 localizes to cell-cell junctions and mediates junctional permeability (Notterpek et al 2001, Roux et al 2005). Similar functions can be assigned to PMP22 in the Schwann cell. PMP22 deficient nerves display disrupted tight and adherens junctions accompanied by increased 
actin polymerization leading to abnormal permeability and decreased mechanical stability of the nerves (Guo et al 2014, Rosso et al 2014). Thereby, the activity of the actin polymerization regulating $p 21$-activated kinase (PAK1) is upregulated and inhibition improves the permeability in PMP22 deficient mice (Hu et al 2016). Recent data shows PMP22 interacting with cholesterol, potentially mediating lipid raft formation (Zhou et al 2019, Zhou et al 2020, Lee et al 2014) and lipid supplementation is beneficial for myelination in PMP22-related diseases (Fledrich et al 2018, Zhou et al 2020). Fledrich et al 2014 showed a PMP22 gene-dosage dependent change in PI3K/AKT/mTOR signaling, PMP22 overexpression leads to a downregulation and PMP22 deficiency to an upregulation of the pathway. In the compact myelin sheath PMP22 interacts with P0, serving as a structural protein in myelin compaction (D'Urso et al 1999). At the abaxonal membrane, PMP22 was shown to indirectly interact with $\beta 4$ integrin (Amici et al 2006, Poitelon et al 2018). Investigating PMP22 protein-protein interaction partners of PMP22 is crucial to further elucidate the molecular function of the protein. Results from our group (Ewers, Arlt, unpublished) detected focal adhesion proteins, such as Vinculin, a potential interaction partners of PMP22.

\section{Vinculin}

Vinculin is a ubiquitously expressed protein localizing to focal adhesions, connecting the cell to the extracellular matrix and to cadherin-mediated cell-cell junctions. Vinculin consists of a head and tail domain, harboring interaction sites for proteins. By binding to actin and adhesion molecules Talin, Catenin, Vinexin and Paxillin, Vinculin regulates migration, adhesion, mobility and spreading of the cell and is involved in transducing mechanical signals (Ziegler et al 2006, Goldmann 2016). Vinculin depletion in mice leads to early embryonic lethality (Xu et al 1998) and conditional deletion in cardiomyocytes results in abnormal adherence junctions in the heart and sudden death in $50 \%$ of the animals during the first three months (ZemljicHarpf et al 2007). Tissue-specific depletion of Vinculin in platelets and astrocytes showed relatively weak phenotypes, suggesting cell-specific roles (Mitsios et al 2010, Winkler et al 2013). Vinculin protein is abundant in the myelin of peripheral nerves (Siems et al 2020) and Vinculin autoantibodies are found in patients suffering from chronic inflammatory demyelination polyneuropathies (CIDP) (Beppu et al 2015), whereas the role in myelination is unknown.

\subsection{Aim of the study}

Alterations in Pmp22 gene-dosage are associated with peripheral neuropathies CMT1A (PMP22 overexpression) and HNPP (PMP22 deficiency). While PMP22 is involved in the regulation of various processes in the cell, the underlying molecular mechanisms remain largely elusive. In order to apply treatment strategies, one has to understand the molecular role of PMP22. Therefore, we aimed at studying (i) possible PMP22 trafficking-mediated stress responses in PMP22 overexpressing rodent models, (ii) the influence of PMP22 dosage on growth signaling in the Schwann cells and (iii) the molecular role of a prospective PMP22 interaction partner. 
Previous studies showed PMP22 retaining in the endoplasmic reticulum upon PMP22 point mutations and strong overexpression (Notterpek et al 1997, Ryan et al 2002). In the first part of this work, we asked the question, does PMP22 overexpression cause ER stress as a primary CMT1A pathomechanism and provide potential therapeutic implications for CMT1A? We investigated expression levels of ER stress markers in nerves of CMT1A rats at different developmental stages and with different disease severity. Moreover, we tested the effects of an ER stress prolonging drug in CMT1A SC-DRG co-cultures in vitro, which was shown to be beneficial in other CMT1 types (Das et al 2015).

Furthermore, Fledrich et al 2014 showed PMP22 gene-dosage dependent alterations in the $\mathrm{PI} 3 \mathrm{~K} / \mathrm{AKT} / \mathrm{mTOR}$ signaling pathway, which is crucial for myelin growth. Using pharmacological and genetic approaches we modulated PI3K/AKT/mTOR signaling in order to improve our understanding on how PMP22 modulates growth signaling in gain and loss of function situations and investigate potential therapeutical targets for HNPP and CMT1A in vitro and in vivo.

To further elucidate the molecular role of PMP22, it is of high interest to investigate proteinprotein interaction partners of PMP22, which are largely unknown. Recent data from our group (Ewers, Arlt, unpublished) suggests focal adhesion protein Vinculin as a potential interaction partner. Therefore, we characterized Vinculin in the Schwann cell and investigated Vinculin-related functions in CMT1A by using primary Schwann cells in vitro and Schwann cell specific Vinculin conditional knockout mice in vivo. 


\section{Materials}

\subsection{Chemicals and Consumables}

All chemicals used, were obtained from Sigma, Merck and SERVA.

\section{Chemicals}

\section{Animals}

Chemical

Company

Ketamine (Ketamin)

WDT

Polyethylenglykol (PEG)

Sigma

Rapamycin

LC Laboratories

Tween 80

Xylazine (Rompun)

Sigma

BayerVital

\section{Cell culture}

\section{Chemical}

Ascorbic acid (AA)

Cytosine $\beta$-D-arabinofuranoside hydrochloride (AraC)

Bovine pituitary extract (BPE)

Collagenase II

Dibutyryladenosine-3', 5' cyclic monophosphate (DBcAMP)

Dulbecco's Modified Eagle's medium, high glucose (4.5 g/l),

pyruvate (-), L-Glutamin(+) (DMEM)

Dimethyl sulfoxide (DMSO)

Fetal calf serum (FCS)

Forskolin (FSK)

GlutaMax ${ }^{\mathrm{TM}}$

IFB-088

Laminin

Minimum Essential Media with L-Glutamine and Earle's salt

(MEM)

Nerve growth factor-2.5S (NGF)

Penicillin/Streptomycin (Pen/Strep)

Polyethyleneimine

Poly-L-Lysine (PLL)

Rapamycin

Trypsin
Company

Sigma

Sigma

Gibco

Worthington

BIOLOG

Gibco

Sigma

HyClone

Sigma

Gibco

InFlectis BioScience

Sigma

Gibco

Alomone Labs

Lonza

Polysciences

Sigma

LC Laboratories

Invitrogen 


\section{Molecular Biology}

Chemical

Acrylamide/ Bisacrylamide

Ammonium persulfate (APS)

Bovine serum albumin (BSA)

Dithiothreitol (DTT)

Ethanol

Fast green

GelCode $^{\mathrm{TM}}$ Blue Stain Reagent

GelRed

GeneRuler 1kb DNA ladder

GoTaq Polymerase

Methanol

Milk powder

PageRuler (Protein marker)

Phosphatase Inhibitors (PhosphoStop)

Protease Inhibitors (Complete Mini)

ProteinaseK

Sodium dodecyl sulfate (SDS)

QIAzol

Tetramethylethylenediamine (TEMED)
Company

Gerbu

BioRad

BioMol

Sigma

Merck

Serva

Thermo Scientific

Biotrend

Fermentas

Promega

Merck

Frema

Thermo Scientific

Roche

Roche

Roth

Sigma

Qiagen

Thermo Scientifc

\section{Histology}

Chemical

Agarose

Azure II

DAPI

Eukitt

Glutardialdehyde

Goat serum

Horse serum

Methylene blue

Mowiol 4-88

Osmium tetraoxide

Paraformaldehyde, pure
Company

Invitrogen

Sigma

Thermo Scientific

Kindler

Electron Microscopy Science

Gibco

Gibco

Sigma

Roth

Science Services

Serva 
Phalloidin-488

Triton-X-100
Invitrogen

Sigma

\section{Kits}

Kit

Biorad Protein Assay Kit

Western Lightning ${ }^{\circledR}$ Plus ECL

Power Sybr ${ }^{\circledR}$ Green PCR Master Mix

RNeasy Mini Kit

Superscript III RT Kit

Function
Protein quantification
HRP Western Blot
detection
q-RT PCR
RNA isolation
cDNA synthesis

Company

Biorad

PerkinElmer

Applied Biosystems

Qiagen

Invitrogen

\section{Consumables}

\begin{tabular}{l|l} 
Consumable & Company \\
Coverslips (glass) & Thermo Scientific Menzel-Gläser \\
Falcon $^{\circledR} 12-$, 24-well plates & Corning Life Sciences \\
Histobonds slides & Marienfeld \\
Immobilon ${ }^{\circledR}$ PVDF membranes & Merck \\
$6 \mathrm{~cm}, 10 \mathrm{~cm}$ Petri dishes & Corning Life Sciences \\
PVDF Western Blotting membranes & Amersham \\
Superfrost Plus slides & Thermo Scientific Menzel-Gläser
\end{tabular}

\subsection{Primer and Antibodies}

\section{Primer}

The in-house AGCT-laboratory of the Max Planck Institute of Experimental Medicine synthesized all oligonucleotide primers.

\section{Genotyping Primers}

\begin{tabular}{l|l|l} 
CPMP & fwd & 5'-CCAGAAAGCCAGGGAACTC -3' \\
CSPP & rev & 5'-GACAAACCCCAGACAGTTG -3' \\
DHHcre & 5'-TCAGGATATCTATCTGATTCTC -3' \\
fwd & 5'-AAGCTCATGGAGCACAAAACC -3' \\
PTENfl & fwd & 5'-CAGCCCGGACCGACGATGAA -3' \\
5'-ACTCAAGGCAGGGATGAGC -3'
\end{tabular}


\begin{tabular}{l|l|l} 
VCLfl & rev & 5'-CAGAGTTAAGTTTTTGAAGGCAAG -3' \\
rev & 5'-TTACGCCTAGCACTTGAA-3‘ \\
& 5'-TGCTCACCTGGCCCAAGATTCTTT-3‘
\end{tabular}

qRT-PCR primers

\begin{tabular}{|c|c|c|}
\hline \multirow[t]{2}{*}{ ATF4 } & fwd & 5'-GGCGTATTAGAGGCAGCAGA-3' \\
\hline & rev & 5‘-CTGCTGGGTTTCGTGAAGAG-3‘ \\
\hline \multirow[t]{2}{*}{ CycloA } & fwd & 5'-TGCTGGACCAAACACAAATG-3’ \\
\hline & Rev & 5'-CACСТTCССAAAGACCACAT-3’ \\
\hline \multirow[t]{2}{*}{ DDIT3 } & fwd & 5’AAGGCACTGAGCGTATCATGT-3’ \\
\hline & & 5`-TGAAGATACACTTCCTTCTTGAACAC-3` \\
\hline \multirow[t]{2}{*}{ GADD34 } & fwd & 5'-GCTTTTGGCAAACCGAAC-3' \\
\hline & rev & 5'-TTCCAGTGCAGGACATGCT-3' \\
\hline \multirow[t]{2}{*}{ HMGCR } & fwd & 5'-GACCTTTCTAGAGCGAGTGCAT-3' \\
\hline & rev & 5'-CGCTATATTCTCССТТАСТTCATCC-3' \\
\hline \multirow[t]{2}{*}{ HSPA5 } & fwd & 5‘-CCGTAACAATCAAGGTCTACGA-3’ \\
\hline & rev & 5'AAGGTGACTTCAATCTGGGGTA-3’ \\
\hline \multirow[t]{2}{*}{ PMP22 } & fwd & 5'-GGCTGTCCCTTTGAACTGAA-3' \\
\hline & rev & 5'-AACAGGATCCCCAACAAGAGT-3' \\
\hline \multirow[t]{2}{*}{ PTEN } & fwd & 5‘-GAGGCCCTGGATTTTTATGG-3’ \\
\hline & rev & 5'-CGCCTCTGACTGGGAATAGT-3’ \\
\hline \multirow[t]{2}{*}{ Rplp0 } & fwd & 5‘-CGAGAAGACCTCTTTCTTCCAA-3’ \\
\hline & rev & 5’AGTCTTTATCAGCTGCACATCG-3’ \\
\hline \multirow[t]{2}{*}{ sXBP1 } & fwd & 5'-GCAAGTGGTGGATTTGGAAG-3’ \\
\hline & rev & 5'AGCCCATGAGTTTTCTCTCG-3’ \\
\hline \multirow[t]{2}{*}{ VCL } & fwd & 5'ACAGTGGATGACCGAGGAGT-3’ \\
\hline & rev & 5`ATGCCCTTCAGCCACAAGT-3’ \\
\hline $\mathrm{dT}$ & $\begin{array}{l}\text { Anchored } \\
\text { oligo-dT } \\
\text { mix }\end{array}$ & TTTTTTTTTTTTTTTTTTTTTTTVN \\
\hline N9 & $\begin{array}{l}\text { Random } \\
\text { nonamers }\end{array}$ & NNNNNNNNN \\
\hline
\end{tabular}

\section{Antibodies}

Primary Antibodies

\begin{tabular}{l|l|l|l} 
Antibody & Species & Company & Dilution \\
Actin & Mouse & Millipore & $1: 200$ (ICC)
\end{tabular}




\begin{tabular}{l|l|l|l} 
AKT & Rabbit & Cell Signaling \#4691 & $1: 1000$ (WB) \\
P-AKT (Ser473) & Rabbit & Cell Signaling \#3787 & $1: 1000$ (WB) \\
DRP2 & Rabbit & $\begin{array}{l}\text { Provided by Peter Brophy } \\
\text { (clone 2164), Sherman et al 2012 }\end{array}$ & $1: 200$ (IHC) \\
MAG & Mouse & Chemicon (clone 513) & $1: 50$ (IHC) \\
MBP & Mouse & Covance \#SMI-99P & $1: 500$ (ICC) \\
NaV1.6 & Rabbit & Alomone labs \#ASC-009 & $1: 250$ (IHC) \\
PMP22 & Rabbit & Assay Biotech C0360 & $1: 100$ (IHC), 1:1000 (WB) \\
PTEN & Rabbit & Cell Signaling \#9188 & $1: 50$ (IHC), 1:1000 (WB) \\
P0 & Mouse & Provided by J.J. Archelos, & $1: 200$ (IHC) \\
S100ß & Rabbit & Abchelos et al 1993 (ab52642) & $1: 200$ (ICC) \\
Talin & Mouse & Sigma clone 8d4 & $1: 250$ (ICC) \\
TUJ1 & Rabbit & Covance & $1: 250$ (ICC), 1:1000 (WB) \\
TUJ1 & Mouse & Covance & $1: 250$ (IHC), 1:1000 (WB) \\
Vinculin & Mouse & Sigma V9264 & $1: 100$ (IHC), 1:1000 (WB)
\end{tabular}

Secondary Antibodies

\begin{tabular}{l|l|l|l} 
Antibody & Species & Company & Dilution \\
Alexa-488 anti mouse & Donkey & Invitrogen & $1: 1000$ (ICC) \\
Alexa-568 anti rabbit & Donkey & Invitrogen & $1: 1000$ (ICC) \\
Alexa-594 anti mouse & Donkey & Invitrogen & $1: 1000$ (STED) \\
HRP anti mouse & Goat & Dianova & $1: 5000(\mathrm{WB})$ \\
HRP anti rabbit & Goat & Dianova & $1: 5000(\mathrm{WB})$ \\
Starred & Rabbit & Aberior & $1: 30$ (STED)
\end{tabular}

\subsection{Plasmids and cell lines}

\section{Plasmids}

\begin{tabular}{l|l|l} 
Name & Fragment/ Mutation & Reference \\
Vcl-eGFP & M1-Q1066 & Chandrasekar et al 2005 \\
Vcl-LD-eGFP & K952Q, K956Q, R963Q, K966Q & Chandrasekar et al 2005
\end{tabular}

Plasmids were kindly provided by Prof. Johannes Hirrlinger, Carl-Ludwig-Institute for Physiology, Leipzig. 


\section{Cell lines}

\begin{tabular}{l|l|l} 
Name & Company & Reference \\
HEK293T & Merck (96121229) & DuBridge et al 1987
\end{tabular}

\subsection{Machines and Software}

\section{Machines}

Device

$4 s^{2}$

AP280

Axio Observer Z.1

AxioCam MRm

Binocular Carl Zeiss

BioRad Electrophoresis

device

Colibri 5

Chemostar PC

Diamond knife Histo $45^{\circ}$

Elevated beam

\section{EMTP}

Evidence 3102evo

FMI-210B2 Force Gauge

HisoStar ${ }^{\mathrm{TM}}$

HeraCell 150

HI 1220

Labofuge 400

LaminAir HBB 2448

Leica TCS SP5

Light Cycler 480

NanoDrop 2000

peqSTAR

Power Pac Basic
Heat sealer

Embedding

Light Microscope

Camera

Binocular

SDS PAGE and Western Blot

LED light source for Light microscopy

Chemiluminescence and

Flourescence Imager

Knife to prepare semithin and ultrathin sections

Elevated beam test

Epon infiltration

Electrophysiological

measurements

Grip strength measurements

Paraffin embedding

Cell culture incubator

Hot Plate

Centrifuge

Laminar flow hoof

Confocal microscope

qRT-PCR machine

Spectraphotometer

PCR machine

Power supply
Company

4titude

Microm

Zeiss

Zeiss

Zeiss

BioRad

Zeiss

Intas

Diamond

Workshop, MPI

Experimental

Medicine

Lynx

Schreiber und Tholen

Medizintechnik

Alluris

Thermo Scientific

Heraeus Instruments

Leica

Heraeus Instruments

Heraeus Instruments

Leica

Roche

Thermo Scientific

PeqLab

BioRad 


\begin{tabular}{l|l|l} 
Precellys & Tissue homogenization & VWR \\
Reichert Ultratrim & Trimmer & Reichert \\
Sled Microtome & Paraffin cross sections & Microm \\
T-Gradient & PCR machine & Biometra \\
Thermomixer comfort & Thermomixer & Eppendorf \\
UV-Systeme & UV Illuminator & Intas \\
Vortex Genie2 & Vortexer & Scientific industries
\end{tabular}

\section{Software}

Software

Adobe Illustrator 2020

Microsoft Excel 2016

FIJI (ImageJ)

Labfolder

Light Cycler 480 software

NanoDrop software

Graphpad Prism 8

Microsoft Word 2016

Zen 2.6 (blue edition)

\begin{tabular}{l|l} 
& Company \\
Figure acquisition & Adobe \\
Data acquisition & Microsoft \\
Image analysis and acquisition & Image \\
Digital lab book & Labfolder \\
qRT PCR data analysis & Roche \\
Protein and mRNA & Thermo \\
concentration & Scientific \\
Statistical analysis and data & Graphpad \\
representation & \\
Text documents & Microsoft \\
Light microscopy & Zeiss
\end{tabular}

\subsection{Solutions and Buffers}

\section{General}

Modified Gritschier (MGB) buffer

$64 \mathrm{mM}$ Tris $\mathrm{HCl} \mathrm{pH} 8.8$

$16.6 \mathrm{mM}$ Ammonium sulfate

$6.5 \mathrm{mM}$ Magnesium chloride

$0.05 \%$ Triton-X-100 [v/v]

2x Phosphate buffer (0.2 M)

$0.36 \%$ monosodium hydogen phosphate $[\mathrm{w} / \mathrm{v}$ ]

$3.1 \%$ disodium hydrogen phosphate [w/v]

$1 \%$ sodium chloride

in $\mathrm{ddH}_{2} \mathrm{O}$

Paraffin fixing solution

$4 \%$ Paraformaldehyde [v/v] 
0.1 M Phosphate buffer

$0.08 \mathrm{M}$ sodium chloride

Adjust $\mathrm{pH}$ to 7.4 .

K\&S fixative (Schultz and Karlsson 1965)

$4 \%$ Paraformaldehyde [v/v]

$2.5 \%$ Glutardialdehyde [v/v]

$0.1 \mathrm{M}$ phosphate buffer

$0.08 \mathrm{M}$ sodium chloride

Adjust $\mathrm{pH}$ to 7.4 .

Phosphate buffered saline (PBS) pH 7.4

$170 \mathrm{mM}$ sodium chloride

$3.4 \mathrm{mM}$ potassium chloride

$4 \mathrm{mM}$ disodium hydrogen phosphate

$1.8 \mathrm{mM}$ dispotassium hydrogen phosphate in $\mathrm{ddH}_{2} \mathrm{O}$

Adjust $\mathrm{pH}$ to 7.4 with $\mathrm{NaOH}$.

\section{Animals}

Anesthetic (Electrophysiology)

4 mg Xylazine (2\% Rompun, Bayer Vital)

$60 \mathrm{mg}$ Ketamine (10\% Ketamin, WDT)

Intraperitoneal injection of $6 \mathrm{mg}$ Ketamine and $90 \mathrm{mg}$ Xylazine per kg bodyweight.

Rapamycin treatment

Stock solution 1:

$10 \%$ polyethyleneglykol [v/v]

$8 \%$ Ethanol $[\mathrm{v} / \mathrm{v}]$

Stock solution 2:

$10 \%$ Tween $80[\mathrm{v} / \mathrm{v}]$

Rapamycin injection solution

Dissolve $100 \mathrm{mg}$ Rapamycin in $2 \mathrm{ml}$ Ethanol (50 mg Rapamycin/ ml)

Dilute 1:50 in Stock solution 1 (1 mg Rapamycin/ ml)

Dilute 1:2 in Stock solution 2 (0.5 mg Rapamycin/ ml)

Placebo injection solution

Dilute Stock solution 1 with stock solution 2 (1:2) 


\section{Cell culture}

Schwann cell-DRG co-culture

Basic medium

$1 \%$ Penicillin/Streptomycin [v/v]

$10 \%$ fetal calf serum

$50 \mathrm{ng} / \mathrm{ml}$ nerve growth factor

in MEM

Myelination medium

$1 \%$ Penicillin/Streptomycin [v/v]

$10 \%$ fetal calf serum

$50 \mathrm{ng} / \mathrm{ml}$ nerve growth factor

$50 \mu \mathrm{g} / \mathrm{ml}$ ascorbic acid

in MEM

Primary Schwann cells

Basic medium

$1 \%$ Penicillin/Streptomycin [v/v]

$1 \%$ GlutaMax [v/v]

$10 \%$ fetal calf serum

in DMEM

Growth medium

$1 \%$ Penicillin/Streptomycin [v/v]

$1 \%$ GlutaMax [v/v]

$10 \%$ fetal calf serum

$4 \mu \mathrm{M}$ foskolin

$100 \mu \mathrm{g} / \mathrm{ml}$ bovine pituitary extract

in DMEM

Stimulation medium

$1 \%$ Penicillin/Streptomycin [v/v]

$1 \%$ GlutaMax [v/v]

$2 \%$ fetal calf serum

1 mM DBcAMP

in DMEM

Cytoskeletal (CSK) buffer

10 mM PIPES

$300 \mathrm{mM}$ sucrose

$3 \mathrm{mM}$ magnesium chloride

$50 \mathrm{mM}$ sodium chloride

$0.1 \%$ Triton-X-100 [v/v]

in $\mathrm{ddH}_{2} \mathrm{O}$ 
HEK cell culture

Growth medium

$$
\begin{aligned}
& 1 \% \text { Penicillin/Streptomycin }[\mathrm{v} / \mathrm{v}] \\
& 5 \% \text { fetal calf serum }
\end{aligned}
$$

\section{Molecular biology}

TBE buffer $\mathrm{pH} 8$ (20x stock solution)

1.8 M Tris base

$1.8 \mathrm{M}$ boric acid

$200 \mathrm{mM}$ EDTA

in $\mathrm{ddH}_{2} \mathrm{O}$

Sucrose buffer

$270 \mathrm{mM}$ sucrose

$10 \mathrm{mM}$ Tris- $\mathrm{HCl} \mathrm{pH} 7.4$

$1 \mathrm{mM}$ sodium hydrogen carbonate

$1 \mathrm{mM}$ magnesium chloride

Protease inhibitor cOmplete tabletsEASY pack

Phosphatase inhibitor PhosStop

Protein loading dye

$40 \%$ glycine $[\mathrm{w} / \mathrm{v}]$

240 mM Tris- $\mathrm{HCl}$ pH 6.8

$8 \%$ SDS [w/v]

$0.04 \%$ bromphenol blue [w/v]

Running buffer

$25 \mathrm{mM}$ Tris base

$192 \mathrm{mM}$ glycine

$1 \%$ SDS [w/v]

Transfer buffer

$48 \mathrm{mM}$ Tris base

$39 \mathrm{mM}$ glycine

$20 \%$ Methanol $[v / v]$

Tris buffered saline with Tween (TBS-T)

$50 \mathrm{mM}$ Tris- $\mathrm{HCl}(\mathrm{pH} 7.4)$

$150 \mathrm{mM}$ sodium chloride

$0.05 \%$ Tween $20[\mathrm{v} / \mathrm{v}]$ 


\section{Fast Green}

Washing solution

$30 \%$ methanol $[\mathrm{v} / \mathrm{v}]$

$6.7 \%$ acetic acid $[v / v] \quad$ in $\mathrm{ddH}_{2} \mathrm{O}$

Staining solution
$30 \%$ methanol $[\mathrm{v} / \mathrm{v}]$
$6.7 \%$ acetic acid $[\mathrm{v} / \mathrm{v}]$
$0.5 \%$ Fast Green $[\mathrm{w} / \mathrm{v}] \quad$ in $\mathrm{ddH}_{2} \mathrm{O}$

\section{Histology}

Immunocytochemistry (ICC) blocking solution

$2 \%$ horse serum

$2 \%$ BSA

$0.1 \%$ gelantine in PBS

Mowiol mounting medium

$24 \%[\mathrm{w} / \mathrm{v}]$ Glycerol

$9.6 \%$ [w/v] Mowiol 4-88

$0.1 \mathrm{M}$ Tris- $\mathrm{HCl}$ pH $8.5 \quad$ in $\mathrm{ddH}_{2} \mathrm{O}$

Paraffin staining

Citrate buffer

A: $\quad 0.1 \mathrm{M}$ citric acid

B: $\quad 0.1 \mathrm{M}$ sodium citrate in $\mathrm{ddH}_{2} \mathrm{O}$

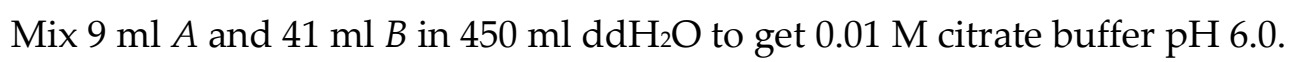

Tris buffered milk

0.05 M TRIS(hydroxymethyl)-aminomethan

in $\mathrm{ddH}_{2} \mathrm{O}$

Adjust $\mathrm{pH}$ to 7.6 with hydrogen chloride

$2 \%$ skim milk powder [w/v]

Stir for 20 minutes and filter before use.

Paraffin blocking buffer

$$
\begin{aligned}
& 10 \% \text { BSA [w/v] } \\
& 20 \% \text { Goat serum [v/v] }
\end{aligned}
$$

in PBS

Teased fiber blocking solution

$$
\begin{aligned}
& 10 \% \text { Horse serum [v/v] } \\
& 1 \% \text { BSA [w/v] } \\
& 0.025 \% \text { Triton-X-100 [v/v] }
\end{aligned}
$$

in PBS 
Gallyas blue staining

Incubation solution

$0.1 \%$ Ammonium nitrate $[\mathrm{w} / \mathrm{v}]$

$0.1 \%$ Silver nitrate $[\mathrm{w} / \mathrm{v}]$

in $\mathrm{ddH}_{2} \mathrm{O}$

Add $3 \mathrm{ml} 4 \%$ sodium hydroxide ( $\mathrm{pH} \mathrm{7,4} \mathrm{-7,6)}$

Developing solution
A: $\quad 5 \%$ Sodium carbonate $[\mathrm{w} / \mathrm{v}]$
in $\mathrm{ddH}_{2} \mathrm{O}$
B: $\quad 0.2 \%$ Ammonium nitrate $[\mathrm{w} / \mathrm{v}]$
$0.2 \%$ Silver nitrate $[\mathrm{w} / \mathrm{v}]$
$1 \%$ Wolframatosilicique acido $[\mathrm{w} / \mathrm{v}]$
in $\mathrm{ddH}_{2} \mathrm{O}$
C: $\quad 0.2 \%$ Ammonium nitrate $[\mathrm{w} / \mathrm{v}]$
$0.2 \%$ Silver nitrate $[\mathrm{w} / \mathrm{v}]$
$1 \%$ Wolframatosilicique acido [w/v]
$0.7 \%$ formol (37 \%) [v/v]
in $\mathrm{ddH}_{2} \mathrm{O}$

$70 \mathrm{ml} B$, then $30 \mathrm{ml} C$ slowly mix to $100 \mathrm{ml} A$.

Etching solution (Maxwell 1978)

Slowly dilute $20 \mathrm{~g}$ potassium hydroxide in $100 \mathrm{ml}$ Methanol (on ice), afterwards add $50 \mathrm{ml}$ propylene oxide and keep stirring 30 minutes on ice.

Methylene blue - Azur II staining solution:

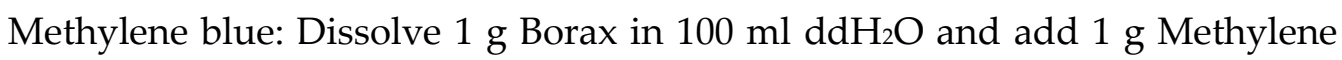
blue.

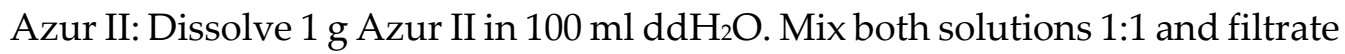
before use. 


\section{Methods}

\subsection{Animals and Behavior}

All animal experiments were performed in accordance with the German animal welfare law and the Lower Saxony State regulations for animal experiments. The animals were kept in the animal facility of the Max Planck Institute of Experimental Medicine in Göttingen, Germany. Housing of the animals took place in cages up to 5 littermates of the gender and, whenever possible, single housing was avoided. Animals had access to water and food ad libitum and were kept in a 12-hour light-dark cycle. We performed all experiments during the light phase.

Table 1 lists all used strains. As rodent models for CMT1A we used the CPMP rat and the C61 mouse model. PMP22 heterozygous knockout mice were used as an animal model for HNPP. In Results part 4.2 we crossbred C61 CMT1A mice with PTEN floxed mice and used DHH as the diver line to specifically reduce half of the PTEN amount in CMT1A Schwann cells. Experiments displayed in Results part 4.3 and 4.4 show results of Vinculin depletion in CMT1A Schwann cells. Experiments were conducted in two to four different groups, which are listed in Table 2. In all experiments, animals of both sexes were used.

Table 1: Rat and mouse lines

\begin{tabular}{|c|c|c|}
\hline Name & MGI Nomenclature & Reference \\
\hline CPMP, CMT1A rat & SD-Tg(Pmp22)Kan & Sereda et al 1996 \\
\hline C61, CMT1A mouse & $\mathrm{Tg}(\mathrm{PMP} 22) \mathrm{C} 61 \mathrm{Clh}$ & Huxley et al 1998 \\
\hline HNPP & Pmp22tm1Ueli & Adlkofer et al 1995 \\
\hline $\mathrm{DHH}$ cre & $\operatorname{Tg}($ Dhh-cre) 1 Mejr/J & Jaegle et al 2003 \\
\hline $\mathrm{PTEN}^{\mathrm{fl} / \mathrm{fl}}$ & Pten $^{\text {tm1Hwu} / J}$ & Groszer et al 2001 \\
\hline $\mathrm{VCL}^{\mathrm{fl} / \mathrm{fl}}$ & $\mathrm{Vcl}^{\mathrm{tm} 1 \mathrm{Ross}} / \mathrm{J}$ & Zemljic-Harpf et al 2007 \\
\hline
\end{tabular}

Table 2: Animal groups

\begin{tabular}{|c|c|c|c|c|c|c|}
\hline Name & $\begin{array}{l}\text { CPMP } \\
\text { PMP22tg }\end{array}$ & $\begin{array}{l}\text { C61,CMT1A } \\
\text { PMP22 }\end{array}$ & $\begin{array}{l}\text { HNPP } \\
\text { PMP22+- }\end{array}$ & $\begin{array}{l}\text { PTEN } \\
\text { PTENfl/fl }\end{array}$ & $\begin{array}{l}\mathrm{VCL} \\
\mathrm{Vcl}^{\mathrm{fl} / \mathrm{fl}}\end{array}$ & $\begin{array}{l}\text { DHH } \\
\text { Dhh }^{\text {cre/+ }}\end{array}$ \\
\hline $\begin{array}{l}\text { WT } \\
\text { CMT1A }\end{array}$ & $\begin{array}{l}\text { wt } \\
\operatorname{tg}\end{array}$ & & & & & \\
\hline $\begin{array}{l}\text { WT } \\
\text { HNPP }\end{array}$ & & & $\begin{array}{l}\text { wt } \\
+/-\end{array}$ & & & \\
\hline $\begin{array}{l}\text { WT } \\
\text { PTENhKO }\end{array}$ & & $\begin{array}{l}w t \\
w t \\
w t\end{array}$ & & $\begin{array}{l}\mathrm{fl} /+ \\
+/+ \\
\mathrm{fl} /+\end{array}$ & & $\begin{array}{l}\text { cre/wt } \\
\text { cre/+ } \\
\text { cre/+ }\end{array}$ \\
\hline
\end{tabular}




\begin{tabular}{l|l|l|l|l|l} 
CMT1A & $\begin{array}{l}\text { tg } \\
\text { tg } \\
\text { tg }\end{array}$ & & $\begin{array}{l}\mathrm{fl} /+ \\
+/+\end{array}$ & $\begin{array}{l}\mathrm{cre} / \mathrm{wt} \\
\mathrm{cre} /+ \\
\mathrm{cre} /+\end{array}$ \\
\hline \hline WT & $\mathrm{wt}$ & & & $\mathrm{fl} / \mathrm{fl}$ & $\mathrm{cre} / \mathrm{wt}$ \\
VclcKO & $\mathrm{wt}$ & & $\mathrm{fl} / \mathrm{fl}$ & $\mathrm{cre} /+$ \\
CMT1A & $\mathrm{tg}$ & & $\mathrm{fl} / \mathrm{fl}$ & $\mathrm{cre} / \mathrm{wt}$ \\
VclcKOxCMT1A & $\mathrm{tg}$ & & $\mathrm{fl} / \mathrm{fl}$ & $\mathrm{cre} /+$
\end{tabular}

\subsubsection{Genotyping}

Animal ear punches were digested in $180 \mu \mathrm{l}$ MGB buffer and $20 \mu \mathrm{l}$ ProteinaseK at $55{ }^{\circ} \mathrm{C}$ with $300 \mathrm{rpm}$ shaking overnight. Afterwards, samples stayed in a $95{ }^{\circ} \mathrm{C}$ water bath for $10 \mathrm{~min}$ to inactivate ProteinaseK. Polymerase chain reactions (PCR) were performed after the following protocols:

\begin{tabular}{|c|c|c|c|c|}
\hline CPMP-PCR & Amount $[\mu \mathrm{l}]$ & Program & & \\
\hline DNA & & $95^{\circ} \mathrm{C}$ & $3 \mathrm{~min}$ & \\
\hline $\mathrm{ddH}_{2} \mathrm{O}$ & & $56^{\circ} \mathrm{C}$ & $30 \mathrm{~s}$ & \\
\hline 5x Buffer & & $72{ }^{\circ} \mathrm{C}$ & $30 \mathrm{~s}$ & $35 x$ \\
\hline dNTPs (2 mM) & & $95^{\circ} \mathrm{C}$ & $30 \mathrm{~s}$ & \\
\hline GoTaq (5 units/ $\mu \mathrm{l}$ ) & & $56^{\circ} \mathrm{C}$ & $60 \mathrm{~s}$ & \\
\hline Primer $11712(10 \mathrm{pmol} / \mu \mathrm{l})$ & & $72{ }^{\circ} \mathrm{C}$ & $5 \mathrm{~min}$ & \\
\hline Primer $11713(10 \mathrm{pmol} / \mu \mathrm{l})$ & & Results & & \\
\hline Primer 2016 (10 pmol/ $\mu \mathrm{l})$ & & wt & $300 \mathrm{bp}$ & \\
\hline Primer $7315(10 \mathrm{pmol} / \mu \mathrm{l})$ & & $\operatorname{tg}$ & $501 \mathrm{bp}$ & \\
\hline CSPP-PCR (C61) & Amount $[\mu l]$ & Program & & \\
\hline DNA & 1 & $94^{\circ} \mathrm{C}$ & $3 \mathrm{~min}$ & \\
\hline $\mathrm{ddH}_{2} \mathrm{O}$ & 10.9 & $56^{\circ} \mathrm{C}$ & $45 \mathrm{~s}$ & \\
\hline $5 x$ Buffer & 4 & $72{ }^{\circ} \mathrm{C}$ & $30 \mathrm{~s}$ & $35 x$ \\
\hline dNTPs (2 mM) & 1 & $95^{\circ} \mathrm{C}$ & $30 \mathrm{~s}$ & \\
\hline GoTaq (5 units/ $\mu \mathrm{l}$ ) & 0.1 & $56^{\circ} \mathrm{C}$ & $60 \mathrm{~s}$ & \\
\hline Primer $11906(10 \mathrm{pmol} / \mu \mathrm{l})$ & 1 & & & \\
\hline Primer $11907(10 \mathrm{pmol} / \mu \mathrm{l})$ & 1 & Results & & \\
\hline Primer $2016(10 \mathrm{pmol} / \mu \mathrm{l})$ & 1 & wt & $700 \mathrm{bp}$ & \\
\hline Primer $7315(10 \mathrm{pmol} / \mu \mathrm{l})$ & 1 & $\operatorname{ctg}$ & $150 \mathrm{bp}$ & \\
\hline
\end{tabular}




\begin{tabular}{|c|c|c|c|c|}
\hline DHHcre-PCR & Amount $[\mu \mathrm{l}]$ & Program & & \\
\hline DNA & 1 & $94^{\circ} \mathrm{C}$ & $90 \mathrm{~s}$ & \\
\hline $\mathrm{ddH}_{2} \mathrm{O}$ & 8.9 & $94^{\circ} \mathrm{C}$ & $30 \mathrm{~s}$ & \\
\hline 5x Buffer & 4 & $58^{\circ} \mathrm{C}$ & $30 \mathrm{~s}$ & $30 x$ \\
\hline dNTPs (2 mM) & 1 & $72{ }^{\circ} \mathrm{C}$ & $30 \mathrm{~s}$ & \\
\hline GoTaq (5 units/ $\mu \mathrm{l}$ ) & 0.1 & $72{ }^{\circ} \mathrm{C}$ & $2 \mathrm{~min}$ & \\
\hline Primer $10967(10 \mathrm{pmol} / \mu \mathrm{l})$ & 1 & & & \\
\hline Primer $15793(10 \mathrm{pmol} / \mu \mathrm{l})$ & 1 & Results & & \\
\hline Primer $2016(10 \mathrm{pmol} / \mu \mathrm{l})$ & 2 & wt & $700 \mathrm{bp}$ & \\
\hline Primer $7315(10 \mathrm{pmol} / \mu \mathrm{l})$ & 2 & cre/+ & $400 \mathrm{bp}$ & \\
\hline PMPZ-PCR (HNPP) & Amount $[\mu l]$ & Program & & \\
\hline DNA & 1 & $95^{\circ} \mathrm{C}$ & $3 \mathrm{~min}$ & \\
\hline $\mathrm{ddH}_{2} \mathrm{O}$ & 9.9 & $95^{\circ} \mathrm{C}$ & $30 \mathrm{~s}$ & \\
\hline $5 x$ Buffer & 4 & $61^{\circ} \mathrm{C}$ & $30 \mathrm{~s}$ & $30 x$ \\
\hline dNTPs (2 mM) & 1 & $72{ }^{\circ} \mathrm{C}$ & $30 \mathrm{~s}$ & \\
\hline GoTaq (5 units/ $\mu \mathrm{l}$ ) & 0.1 & $72{ }^{\circ} \mathrm{C}$ & $5 \mathrm{~min}$ & \\
\hline Primer $11712(10 \mathrm{pmol} / \mu \mathrm{l})$ & 0.5 & & & \\
\hline Primer $11713(10 \mathrm{pmol} / \mu \mathrm{l})$ & 0.5 & Results & & \\
\hline Primer 2016 (10 pmol/ $\mu \mathrm{l})$ & 2 & wt & $700 \mathrm{bp}$ & \\
\hline Primer $7315(10 \mathrm{pmol} / \mu \mathrm{l})$ & 2 & $\operatorname{tg}$ & $260 b p+7$ & $700 \mathrm{bp}$ \\
\hline PTENflox-PCR & Amount $[\mu \mathrm{l}]$ & Program & & \\
\hline DNA & 1 & $95^{\circ} \mathrm{C}$ & $3 \mathrm{~min}$ & \\
\hline $\mathrm{ddH}_{2} \mathrm{O}$ & 12.9 & $60^{\circ} \mathrm{C}$ & $30 \mathrm{~s}$ & \\
\hline 5x Buffer & 4 & $72{ }^{\circ} \mathrm{C}$ & $60 \mathrm{~s}$ & $33 x$ \\
\hline dNTPs (2 mM) & 2 & $95^{\circ} \mathrm{C}$ & $30 \mathrm{~s}$ & \\
\hline GoTaq (5 units/ $\mu \mathrm{l}$ ) & 0.1 & $60^{\circ} \mathrm{C}$ & $60 \mathrm{~s}$ & \\
\hline Primer $5495(10 \mathrm{pmol} / \mu \mathrm{l})$ & 0.5 & $72{ }^{\circ} \mathrm{C}$ & $10 \mathrm{~min}$ & \\
\hline \multirow[t]{4}{*}{ Primer $20515(10 \mathrm{pmol} / \mu \mathrm{l})$} & 0.5 & & & \\
\hline & & Results & & \\
\hline & & $w t$ & $202 \mathrm{bp}$ & \\
\hline & & flox/flox & $374 \mathrm{bp}$ & \\
\hline
\end{tabular}




\begin{tabular}{|c|c|c|c|}
\hline VCLflox-PCR & Amount $[\mu l]$ & Program & \\
\hline DNA & 1 & $94^{\circ} \mathrm{C}$ & $30 \mathrm{~s}$ \\
\hline $\mathrm{ddH}_{2} \mathrm{O}$ & 11.9 & $54^{\circ} \mathrm{C}$ & $30 \mathrm{~s}$ \\
\hline 5x Buffer & 4 & $72^{\circ} \mathrm{C}$ & $30 \mathrm{~s}$ \\
\hline dNTPs (2 mM) & 2 & $94^{\circ} \mathrm{C}$ & $30 \mathrm{~s}$ \\
\hline GoTaq (5 units/ $\mu$ l) & 0.1 & $54{ }^{\circ} \mathrm{C}$ & $30 \mathrm{~s}$ \\
\hline Primer $37592(10 \mathrm{pmol} / \mu \mathrm{l})$ & 1 & $72{ }^{\circ} \mathrm{C}$ & $10 \mathrm{~min}$ \\
\hline \multirow[t]{4}{*}{ Primer $37593(10 \mathrm{pmol} / \mu \mathrm{l})$} & 1 & & \\
\hline & & Results & \\
\hline & & & $1.7 \mathrm{~kb}$ \\
\hline & & flox/flox & $1.5 \mathrm{~kb}$ \\
\hline
\end{tabular}

PCR products were separated on $1.5 \%$ agarose gels (VCLflox PCR $1 \%$ ) in TBE buffer with GelRed 1:20 000 .

\subsubsection{Rapamycin treatment}

HNPP and control mice were i.p. injected with $5 \mathrm{mg}$ Rapamycin per $\mathrm{kg}$ bodyweight two times per week from postnatal day 21 until postnatal day 148 with either Rapamycin or Placebo injection solution. The weight was continuously controlled and animals were subjected to behavioral tests and electrophysiology at the end of the study.

Rapamycin treatment was performed by Sandra Göbbels and colleagues at the Max Planck Institute of Experimental Medicine in the Department of Neurogenetics.

\subsubsection{Behavior}

Motor and sensory behavior was assessed in three different tests. To evaluate hind- and forelimb grip strength we performed the Grip Strength test. For motor coordination, animals were analyzed in the Elevated beam. The Hotplate test assessed the animal's thermal sensory reaction.

\section{Grip Strength:}

Holding the animals by their tail they were placed with their forelimbs on a horizontal bar. By gently pulling the animal away, the maximum force was measured in a connected gauge. To assess hind limb grip strength, the animal's forelimbs were placed on a small rectangular plastic plate and their hindlimbs on the bar. Again, the animal was retracted from the bar and the gauge detected the maximum force applied. All measurements were repeated seven times per animal and the mean calculated. In between fore- and hindlimb grip strength analysis, the animals had a minimum break of 10 minutes.

\section{Elevated beam test:}

The elevated beam is an $80 \mathrm{~cm}$ long, $14 \mathrm{~mm}$ wide bar approximately $60 \mathrm{~cm}$ above the ground. At the end of the slightly upwards pointing bar, a little box is located. We trained the animals 
one day prior to their experimental day, so they get used to the test and run up to the box without interruption. We evaluated the time the animals needed to pass the $80 \mathrm{~cm}$ bar and the number of slips occurring on their way. All animals walked the elevated beam for three times and the mean time and number of slips was calculated.

\section{Hotplate:}

Animals were placed on a $55^{\circ} \mathrm{C}$ warm plate surrounded by a Plexiglas chamber. We measured the time until they showed a nociceptive reaction for instance jumping, licking or retracting of hindlimbs. The Hotplate was the final behavior test and not trained beforehand.

All tests were carried out blinded to the animal's genotype and according to established standardized methods in the laboratory.

\subsubsection{Electrophysiology}

For anesthesia, mice were injected i.p. with Ketamine/ Xylazine. When no toe reflexes were observed anymore, electrophysiological measurements on the sciatic/ tibial nerve and tail were performed. Therefore, needle electrodes were subcutaneously applied close to the sciatic notch (proximal stimulation) and in close proximity to the ankle (distal stimulation). Motor recording electrodes were inserted in the small muscle on the plantar surface (Figure VIa). After proximal and distal supramaximal stimulation the compound muscle action potential (CMAP) was recorded. The distance between the stimulation sites [m] divided by the difference of latencies [s] allowed to calculate the motor nerve conduction velocity (mNCV) (Figure VIa).

In addition to the M-wave provoked by orthodromic impulse propagation after distal nerve stimulation, the antidromic signal propagation reaches the motorneuron soma in the ventral horn of the spinal cord which backfires and evokes a second muscle action potential the Fwave, thus representing the entirety of the motor unit. F-wave measurements are means of 10
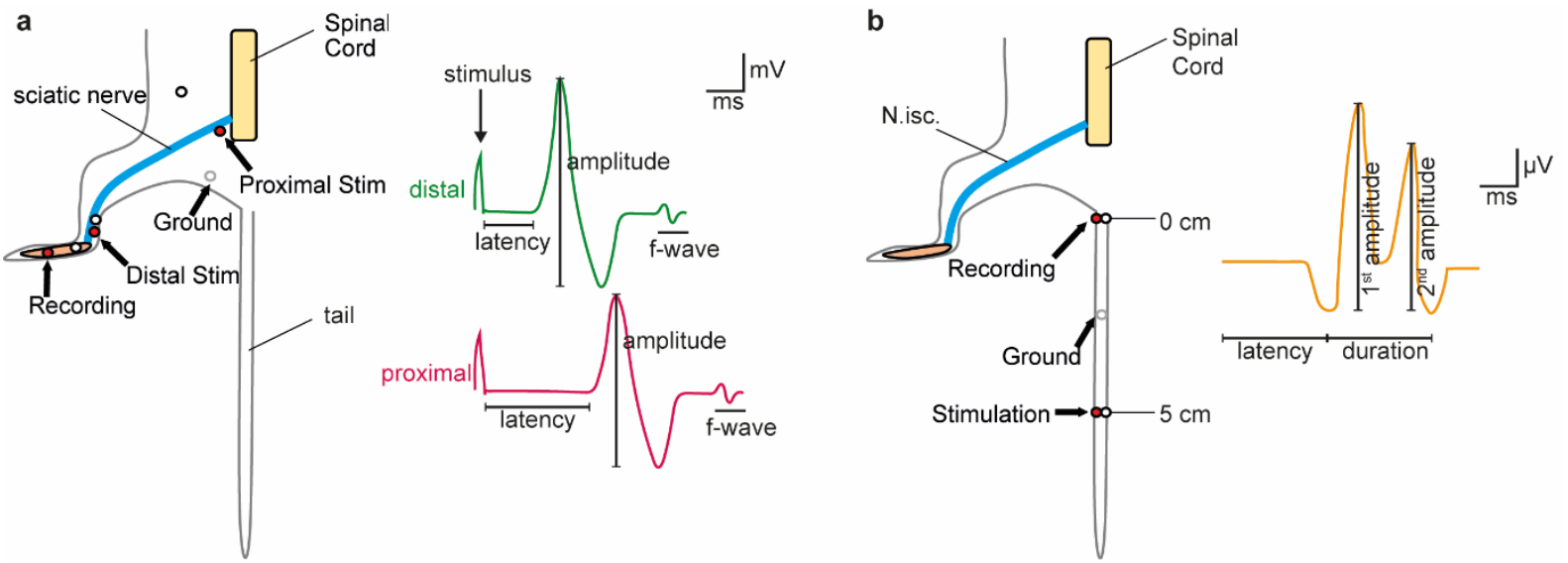

Figure VI: Electrophysiological measurements in the mouse.

(a) Left panel shows setup of motor measurements in the sciatic/tibial nerve. The right panel displays typical motor recordings after distal (green) and proximal (pink) stimulation.

(b) Left panel shows setup of sensory measurements in the sciatic/tibial nerve. The right panel displays typical sensory recording (orange).

The active electrode is displayed in red, the reference electrode in white. 
recordings with a frequency of $0.3 \mathrm{~Hz}$ and a stimulation of $3 \mathrm{~mA}$. Repetitive stimulations are bursts of 10 stimuli at frequencies of $3 \mathrm{~Hz}, 10 \mathrm{~Hz}$ and $50 \mathrm{~Hz}$ with a supramaximal stimulation of $3 \mathrm{~mA}$. The decrement between first and last CMAP was calculated to assess exhaustion of the given frequency.

For sensory measurements in the tail, needle electrodes were applied close to the tip of the tail as stimulation electrodes and electrodes in the proximal tail branch served as recording electrodes (Figure VIb). Averaged compound sensory nerve potentials were measured after stimulation with $5 \mathrm{~mA}$. According to the distance of electrodes the sensory nerve conduction velocity (sNCV) was calculated (Figure VIb).

Electrophysiology was performed by Theresa Kungl and Robert Fledrich.

\subsubsection{Tissue preparation}

\section{Peripheral nerves}

Animals were sacrificed by cervical dislocation or decapitation (up to postnatal day 6). Afterwards Nerous ischiadici, Nervus tibiali as well as Nerous femorali and Nervus sapheni were dissected on both sides. For immunohistochemistry, one femoral and saphenous nerve was immersion fixed in $4 \%$ PFA for $24 \mathrm{~h}$. The other femoral and saphenous nerve was incubated in K\&S fixative for at least one week and further processed for electron microscopy. The epineurium of both tibial nerves and one sciatic nerve was carefully removed and the tissue snap frozen and stored at $-80{ }^{\circ} \mathrm{C}$ until further use for either RNA or protein analysis. The remaining sciatic nerve was transferred to ice cold PBS and then further processed to teased fibers.

\section{Teased fibers}

To prepare teased fibers, the connective tissue was carefully removed using fine forceps. Afterwards, the nerve was longitudinally divided into five parts (three parts at postnatal day 6 and 18). Each part was split in a drop of ice cold PBS on a superfrost slide to get single axons. The samples were dried for about $15 \mathrm{~min}$ at room temperature and stored at $-20^{\circ} \mathrm{C}$ until further processing.

\subsection{Cell culture}

\subsubsection{Schwann cell-DRG co-culture}

\section{Rat tail collagen coating}

Rat tail collagen was mixed in a 1:1 ratio with $0.001 \%$ acetic acid. Afterwards, one drop of the solution was placed on each coverslip and distributed with a small glass spatula. The plates were kept under the laminar flow hood to dry before use. 


\section{Schwann cell-dorsal root ganglia co-culture}

Dorsal root ganglia (DRG) cultures were prepared from either E15.5 rat or E13.5 mouse embryos. Rats were sacrificed by $\mathrm{CO}_{2}$ inhalation and mice by cervical dislocation. The abdomen was opened up and the uterus removed. Afterwards, the single embryos were dissected and decapitated. Under the binocular, the embryos inner organs were removed and the Spinal cord and DRGs exposed. The surrounding tissue was removed and the DRGs carefully detached from the spinals cord. The DRGs of each embryo were separately collected in MEM medium. Next, the cells were pelleted by $5 \mathrm{~min}$ centrifugation at $800 \mathrm{rpm}$. The supernatant was discarded and the cells resuspended in $300 \mu 10.25 \%$ Trypsin and digested at $37^{\circ}$ for $45 \mathrm{~min}$. Digestion was stopped by adding $300 \mu \mathrm{l} \mathrm{FCS}$ and $1 \mathrm{~mL}$ DRG basic medium. Cells were titrated 10 times in heat sealed glass pipettes to separate properly. Another centrifugation step for $8 \mathrm{~min}$ at $800 \mathrm{rpm}$ followed. The supernatant was discarded and the cells were taken up in $160 \mu \mathrm{l}$ basic medium and plated in $40 \mu \mathrm{l}$ drops on rat tail collagen coated coverslips (4 coverslips per embryo on 12 well plate). The next day the medium was filled up with $1 \mathrm{ml}$ basic medium. On day 7, myelination was induced by adding DRG myelination medium to the cultures. For PTEN inhibition in CMT1A cultures, one coverslip per embryo was treated with DMSO as a control and the other three coverslips were treated with a specific PTEN inhibitor (VO-OHpic; Rosivatz et al 2006) at $50 \mathrm{nM}, 500 \mathrm{nM}$ and $5 \mu \mathrm{M}$. In HNPP cultures, cell were treated with $20 \mathrm{nM}$ Rapamycin. The DRG myelination medium (plus inhibitor) was changed every 2-3 days for two weeks.

\subsubsection{Primary Schwann cells}

\section{PLL coating of tissue culture dishes}

Firstly, PLL ( $5 \mathrm{mg} / \mathrm{ml}$ ) was diluted in $\mathrm{ddH}_{2} \mathrm{O}$ to reach $100 \mu \mathrm{g} / \mathrm{ml}$. Afterwards, PLL was poured in the needed dishes (with our without coverslips) until covering the whole bottom of the dish. The dishes were incubated at $37^{\circ} \mathrm{C}$ for at least $30 \mathrm{~min}$. Later, PLL was removed and dishes washed with $\mathrm{ddH}_{2} \mathrm{O}$ for three times and dishes were kept under the laminar flow hood until dried.

\section{Laminin coating of tissue culture dishes}

Laminin coating was performed directly before cells were seeded on the coated plates/ coverslips. First, laminin was diluted in DMEM to reach a concentration of $10 \mu \mathrm{g} / \mathrm{ml}$. For dishes, laminin solution was poured on PLL coated culture dishes, so the solution thinly covered the dish surface. For coverslips, small laminin solution drops were placed in the middle of the coverslip. After incubation for at least $30 \mathrm{~min}$ at $37^{\circ} \mathrm{C}$, laminin solution was carefully removed and cells directly seeded on the still wet plates.

\section{Primary Schwann cell preparation}

Primary Schwann cells were prepared from 2-4 days old rats or mice. Therefore, rats were sacrificed by decapitation and sciatic nerves dissected and transferred to ice cold DMEM. In 
the next step the connective tissue was removed and the nerves of 4-6 animals of the same genotype placed in $300 \mu \mathrm{l}$ trypsin and $300 \mu \mathrm{l}$ collagenase II on a $60 \mathrm{~mm}$ dish. The dish was placed in the incubator $\left(37^{\circ} \mathrm{C}, 5 \% \mathrm{CO}_{2}\right)$ for 1 hour, while kept on an angle. For dissociation of the cells, nerves were triturating 10 times through a $1 \mathrm{ml}$ pipette tip and 10 times through a $200 \mu \mathrm{l}$ pipette tip before digestion was stopped by adding $1 \mathrm{ml} \mathrm{FCS}$ and $3 \mathrm{ml}$ DMEM. After 10 min centrifugation at $1000 \mathrm{rpm}$ cells, were resuspended in pre-warmed SC basic medium and transferred to PLL-coated tissue culture dishes. On the next day, media was changed to SC basic medium containing $10 \mu \mathrm{M}$ Cytosine $\beta$-D-arabinofuranoside hydrochloride (AraC) to eliminate fibroblasts. After three days media was removed and cells kept in SC growth medium until ready for splitting.

\section{Schwann cell splitting}

When the Schwann cells grow to a confluent monolayer on the tissue dish, media was removed and cells washed with PBS. To detach the cells, $10 \%$ Trypsin in PBS (2 ml on $10 \mathrm{~cm}$ culture dish) was added and cells incubated for $2-3 \mathrm{~min}$ at $37^{\circ} \mathrm{C}$. After centrifugation for $5 \mathrm{~min}$ at 800 rpm supernatant was discarded and cell pellet resuspended in pre-warmed SC growth medium before separating cells on the needed tissue culture dishes. For all experiments, Schwann cells between passage one and four (P1-P4) were used.

\subsubsection{Transfection of HEK293T cells}

HEK293T cells were either grown on $10 \mathrm{~cm}$ petri dishes (for protein lysate) or 12 well plates (for immunofluorescent staining) in HEK cell growth medium until $80 \%$ confluence. Then, cells were transfected using Polyethyleneimine (PEI) (Huh et al 2007). Therefore, plasmid DNA was mixed with PBS and PEI was mixed with $\mathrm{ddH}_{2} \mathrm{O}$ according to the plate size:

\begin{tabular}{l|l|l|l|l} 
Plate size & DNA $[\mu \mathrm{g}]$ & PBS $[\mu \mathrm{l}]$ & PEI $[\mu \mathrm{l}]$ & $\mathrm{ddH} \mathrm{H}_{2} \mathrm{O}[\mu \mathrm{l}]$ \\
12 well & 1.33 & 80 & 0.53 & 80 \\
$10 \mathrm{~cm}$ & 14.8 & 892 & 11.9 & 892
\end{tabular}

Afterwards, PEI and DNA solutions were pipetted together and incubated at room temperature for 30 minutes. The transfection solution was then dropwise added on the medium of the cells. After $48 \mathrm{~h}$ of incubation, transfected HEK cells were either harvested in protein lysate buffer of fixed for subsequent immunofluorescent staining.

\subsubsection{Scratch Assay}

To assess the migration speed of primary Schwann cells, Scratch Assay experiments were performed as described in Lee et al 2014. Therefore, primary Schwann cells were plated in PLL coated 12 well plates and kept in Schwann cell growth media until they formed a confluent layer. In the next step, the media was removed and cells washed with pre-warmed PBS before applying DMEM and stepwise reducing FCS from $5 \%$ to $2 \%$ with PBS washing steps in 
between over one day. Afterwards, cells were washed two times with DMEM. Using a $200 \mu 1$ tip a horizontal Scratch was created in the Schwann cell monolayer. To remove loose cells, they were washed three times with DMEM. Subsequently, cells were kept either in SC basic medium with $2 \%$ FCS or SC stimulation medium. The cells were monitored in a live cell imaging setup for 8 hours and afterwards the percentage of the migrated area into the scratch calculated using FIJI image analysis software.

\subsection{Molecular biology}

\subsubsection{RNA isolation and qRT-PCR}

For RNA analysis in tissue, we used snap frozen tibial nerves which had to be homogenized and for RNA analysis SC-DRG co-culture, cells were taken up in RLT buffer and frozen until use without further homogenization.

\section{Homogenization and RNA extraction}

For total RNA extraction from tissue, one tibial nerve (without epineurium) was used and placed in $1000 \mu \mathrm{l}$ QIAzol ${ }^{\circledR}$ lysis reagent and homogenized in the Precellys $6000 \mathrm{rpm}$ two times for 15 s. RNA from cell culture was purified by taking up the cells in RLT lysis buffer. Afterwards RNA was purified according to manufacturer's instructions using RNeasy Kit (Qiagen). The concentration and purity of the RNA was determined with the ratio of absorption at 260/280 nm using NanoDrop 2000 Spectraphotometer.

\section{cDNA synthesis}

To compare relative mRNA amounts, $400 \mathrm{ng}$ of isolated RNA was reversely transcribed to cDNA following the protocol below:

cDNA synthesis

Amounts

400 ng RNA

$0.6 \mu \mathrm{M}$ Oligo dT mix primer

$0.12 \mathrm{mM}$ N9 random nonamer $2 \mu \mathrm{l}$ primer

$\begin{array}{ll}5 \times 1^{\text {st }} \text { strand buffer } & 8 \mu \mathrm{l} \\ 100 \mathrm{mM} \text { DTT } & 4 \mu \mathrm{l} \\ 10 \mathrm{mM} \text { dNTPs } & 1 \mu \mathrm{l} \\ \text { SuperScript }^{\circledR} \text { III reverse } & 1 \mu \mathrm{l} \\ \text { transcriptase } & \\ (200 \mathrm{U} / \mu \mathrm{l}) & \end{array}$

\section{Program}

$8 \mu \mathrm{l} \quad$ Incubate at $70{ }^{\circ} \mathrm{C}$ for $2 \mathrm{~min}$ for denaturation of

$2 \mu \mathrm{l}$ double stranded RNAs and primers.

Afterwards, put on ice. 
Newly synthesized cDNA was diluted to $1 \mathrm{ng} / \mu \mathrm{l}$ in $\mathrm{ddH}_{2} \mathrm{O}$ and stored at $-20{ }^{\circ} \mathrm{C}$ until use for qRT-PCR.

\section{Quantitative real time polymerase chain reaction (qRT-PCR)}

For comparison of relative mRNA amounts in tissue or cell culture, qRT-PCR was performed using the SybrGreen system. For each reaction, $2 \mu \mathrm{cDNA}$ were mixed with $5 \mu$ l Power SybrGreen master mix and $0.2 \mu \mathrm{l}$ forward as well as reverse primer $(100 \mu \mathrm{M})$. Per reaction four technical replicates were analyzed.

qRT-PCR Program

\begin{tabular}{l|l|l|l} 
Warm up & $50^{\circ} \mathrm{C}$ & $120 \mathrm{~s}$ & \\
Melting & $95^{\circ} \mathrm{C}$ & $600 \mathrm{~s}$ & \\
Annealing & $60^{\circ} \mathrm{C}$ & $20 \mathrm{~s}$ & \\
Elongation and detection & $72^{\circ} \mathrm{C}$ & $30 \mathrm{~s}$ & $45 \mathrm{x}$ \\
Melting & $95^{\circ} \mathrm{C}$ & $10 \mathrm{~s}$ &
\end{tabular}

Reaction was performed in 384 well plates and measured in the Light Cycler 480. The mean cycle threshold (ct) of four technical replicates was analyzed by the according software and normalized to cyclophilin $\mathrm{A}(\mathrm{cyclo} A)$ and ribosomal large $\mathrm{P0}(r p l p 0)$ expression using the deltadelta-ct (ddct) method in Excel.

\subsubsection{Protein lysate extraction and quantification}

Protein lysates were produced from sciatic nerves without epineurium by homogenizing the tissue in $270 \mathrm{mM}$ sucrose buffer using the Precellys (3x $6000 \mathrm{rpm}$ for $15 \mathrm{~s}$ ). Protein concentrations were either measured in the NanoDrop 2000 using the absorption of aromatic amino acids, such as Tryptophan, at $280 \mathrm{~nm}$ or the BioRad DC ${ }^{\mathrm{TM}}$ Protein Assay system according to producer's protocol, based on a BSA standard curve.

\subsubsection{Myelin purification}

Myelin purification was performed by David Ewers following a protocol by Larocca and Norton 2006. In short, $\mathrm{n}=6$ dissected sciatic nerves of rats at postnatal day 18 were homogenized in $0.3 \mathrm{M}$ sucrose (PreCellys, $6000 \mathrm{rpm}, 2 \times 15 \mathrm{~s}$ ). Part of the homogenate was kept as 'lysate control' and the remaining homogenate was layered over $0.83 \mathrm{M}$ sucrose in centrifugation tubes and centrifuged at $75000 \times \mathrm{g}$ for $30 \mathrm{~min}$ at $4{ }^{\circ} \mathrm{C}$ (Ultracentrifuge, TLS55 rotor). The interface between the sucrose gradients (Myelin) was carefully transferred to a new centrifugation tube with Tris- $\mathrm{Cl}$ buffer and again centrifuged at $75000 \mathrm{xg}$ for $15 \mathrm{~min}$ at $4{ }^{\circ} \mathrm{C}$. The pellet was resuspended in Tris- $\mathrm{Cl}$ buffer solution and centrifuged for a second time at 12 $000 \mathrm{x} g$ for $15 \mathrm{~min}$. Furthermore the pellet was taken up in $0.3 \mathrm{M}$ sucrose and layered over 0.83 $\mathrm{M}$ sucrose for a second density gradient centrifugation at $75000 \mathrm{xg}$ for $30 \mathrm{~min}$ at $4{ }^{\circ} \mathrm{C}$. The interface was collected again and one more time centrifuged in Tris- $\mathrm{Cl}$ buffer solution at 75 
$000 \times g$ for $15 \mathrm{~min}$. The pellet contains the enriched myelin fraction and was further processed for Western Blot analysis.

\subsubsection{SDS-PAGE and Western Blot}

\section{Protein separation with SDS-PAGE}

$12 \%$ SDS gel

Running gel:

Stacking gel

\begin{tabular}{|l|l|}
$12 \%[\mathrm{v} / \mathrm{v}]$ & Acrylamide/ Bisacrylamide 29:1 \\
$0.4 \mathrm{M}$ & Tris $\mathrm{HCl} \mathrm{pH} 8.8$ \\
$0.1 \%[\mathrm{w} / \mathrm{v}]$ & SDS \\
$0.03 \%[\mathrm{v} / \mathrm{v}]$ & Ammonium persulfate \\
$0.08 \%[\mathrm{v} / \mathrm{v}]$ & TEMED
\end{tabular}

\begin{tabular}{|l|l}
$4 \%[\mathrm{v} / \mathrm{v}]$ & Acrylamide/ Bisacrylamide 29:1 \\
$125 \mathrm{mM}$ & Tris $\mathrm{HCl} \mathrm{pH} 6.8$ \\
$0.1 \%[\mathrm{w} / \mathrm{v}]$ & SDS \\
$0.05 \%[\mathrm{v} / \mathrm{v}]$ & Ammonium persulfate \\
$0.1 \%[\mathrm{v} / \mathrm{v}]$ & TEMED
\end{tabular}

Whole sciatic nerve lysate, cell culture lysate or purified myelin was mixed with protein loading dye containing $1 \mathrm{mM}$ DTT as reducing agent and heated to $50^{\circ} \mathrm{C}$ for 10 minutes. Either

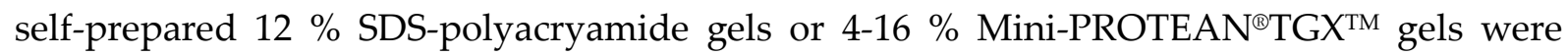
loaded with 5 - $20 \mu \mathrm{g}$ protein and $4 \mu \mathrm{l}$ PageRuler as a marker and run at $\sim 75 \mathrm{~V}$ until proteins were focused in the stacking gel. Afterwards, voltage was increased to $\sim 120 \mathrm{~V}$ until proteins were fully separated according to their size.

\section{Coomassie}

For protein detection after SDS-PAGE, gels were shortly washed in water for three times and then incubated in GelCode ${ }^{\mathrm{TM}}$ Blue Stain Reagent (Thermo Scientific), which is based on colloidal coomassie dye G-250, for 60 minutes. Afterwards gels were de-stained in water over night and then imaged using the Intas Chemostar.

\section{Protein transfer by Western Blot}

For analysis of specific Protein amounts in the protein lysates, the size-separated proteins have to be transferred to a membrane for antibody detection. Therefore, PVDF membranes were activated in methanol for one minute, shortly washed in water and incubated in transfer buffer for at least one minute. Transfer was performed using the wet chamber from Biorad. Thus, gel and membrane were stacked in a sandwich of filter and filter membrane before set in the Biorad apparatus, which was filled up with cold transfer buffer. Depending on the size of the protein of interest, transfer was run between 1 and 2 hours by $65-100 \mathrm{~V}$ at $4{ }^{\circ} \mathrm{C}$. 


\section{Fast green whole protein staining}

After transfer, membranes were shortly washed in water and stained for whole protein amount in fast green staining solution for 5 minutes. In the next step, membranes were two times washed in fast green washing solution for 30 seconds. Fast green unspecifically binds proteins and shows its maximum absorption at $\sim 620 \mathrm{~nm}$. Fluorescence of the stained membrane was measured using the Intas Chemostar. Next, membrane were at least three times washed in TBS-T buffer before proceeding with specific protein analysis by antibodies.

\section{Specific protein detection by antibodies}

Membranes were blocked against unspecific antibody binding for one hour in either $5 \%$ MilkTBS-T or $5 \%$ BSA-TBS-T (Phospho-Antibodies). Afterwards, membranes were incubated with primary antibodies in blocking solution over night at $4{ }^{\circ} \mathrm{C}$. The next day, the membranes were washed three times for five minutes in TBS-T before incubation with HRP coupled secondary antibodies in blocking solution for 45 minutes at room temperature. Subsequently membranes were washed at least four times in TBS-T before detection. Therefore, membranes were covered in Western Lightning Plus-ECL-Kit developing solution and imaged using the Intas Chemostar. Relative densities were assessed using FIJI and normalization was performed on either whole protein amounts or standard protein TUJ1.

\subsection{Histology}

\subsubsection{Tissue embedding}

\section{Paraffin wax embedding}

Femoral and saphenous nerve were embedded in paraffin wax to make cross sections for fluorescent stainings. Therefore, PFA fixed nerves were placed in $2 \%$ agarose in PBS and afterwards in Paraffin using the HistoStar ${ }^{\mathrm{TM}}$ (Thermo Scientific). Embedding was done following the protocol below:

\begin{tabular}{l|l|l} 
Step & Repetition & Duration \\
$50 \%$ Ethanol & $1 \mathrm{x}$ & $1 \mathrm{~h}$ \\
$70 \%$ Ethanol & $2 \mathrm{x}$ & $2 \mathrm{~h}$ \\
$96 \%$ Ethanol & $2 \mathrm{x}$ & $1 \mathrm{~h}$ \\
$100 \%$ Ethanol & $2 \mathrm{x}$ & $1 \mathrm{~h}$ \\
2-Propanol & $1 \mathrm{x}$ & $1 \mathrm{~h}$ \\
Xylol & $2 \mathrm{x}$ & $2 \mathrm{~h}$ \\
Paraffin wax $\left(60^{\circ} \mathrm{C}\right)$ & $2 \mathrm{x}$ & $2 \mathrm{~h}$
\end{tabular}

After embedding in paraffin was, $5 \mu \mathrm{m}$ microtome cross sections were performed. Tissue was transferred to slides and dried at $37^{\circ} \mathrm{C}$ over night. 


\section{Epoxy resin embedding}

Femoral and saphenous nerve were embedded in Epoxy resin to produce semi-thin and ultrathin cross sections for Gallays blue staining and Electron microscopy. Hence, nerves were packed in filter paper and put in little baskets and embedded following this protocol:

\begin{tabular}{l|l|l|l} 
Step & Repetition & Duration & Temperature \\
Phosphate Buffer (PB) $(0.1 \mathrm{M})$ & $3 \mathrm{x}$ & $10 \mathrm{~min}$ & $4{ }^{\circ} \mathrm{C}$ \\
Osmium tetraoxide (2\% in 0.1 M PB) & $1 \mathrm{x}$ & $4 \mathrm{~h}$ & $4{ }^{\circ} \mathrm{C}$ \\
Phosphate Buffer (PB) $(0.1 \mathrm{M})$ & $3 \mathrm{x}$ & $10 \mathrm{~min}$ & $4{ }^{\circ} \mathrm{C}$ \\
$30 \%$ Ethanol & $1 \mathrm{x}$ & $20 \mathrm{~min}$ & $4{ }^{\circ} \mathrm{C}$ \\
$50 \%$ Ethanol & $1 \mathrm{x}$ & $20 \mathrm{~min}$ & $4{ }^{\circ} \mathrm{C}$ \\
$70 \%$ Ethanol & $1 \mathrm{x}$ & $20 \mathrm{~min}$ & $4{ }^{\circ} \mathrm{C}$ \\
$90 \%$ Ethanol & $1 \mathrm{x}$ & $20 \mathrm{~min}$ & $4{ }^{\circ} \mathrm{C}$ \\
$100 \%$ Ethanol & $3 \mathrm{x}$ & $10 \mathrm{~min}$ & $4{ }^{\circ} \mathrm{C}$ \\
2-Propanol & $1 \mathrm{x}$ & $10 \mathrm{~min}$ & $4{ }^{\circ} \mathrm{C}$ \\
Propylene oxide & $3 \mathrm{x}$ & $10 \mathrm{~min}$ & $\mathrm{RT}$ \\
2:1 Propylene oxide : Epon & $1 \mathrm{x}$ & $2 \mathrm{~h}$ & $\mathrm{RT}$ \\
1:1 Propylene oxide : Epon & $1 \mathrm{x}$ & $2 \mathrm{~h}$ & $\mathrm{RT}$ \\
1:2 Propylene oxide : Epon & $1 \mathrm{x}$ & $4 \mathrm{~h}$ & $\mathrm{RT}$ \\
Pure Epon & $1 \mathrm{x}$ & $4 \mathrm{~h}$ & $\mathrm{RT}$
\end{tabular}

Tissues were taken out of the basket and transferred to embedding molds and let polymerize for $24 \mathrm{~h}$ at $60^{\circ} \mathrm{C}$.

\subsubsection{Fluorescent staining}

\section{SC-DRG co-culture}

SC-DRG co-cultures were fixed in $4 \%$ PFA for 10 minutes. Afterwards, cells were permeabilized in ice-cold Methanol-Aceton (95/5) for 5 minutes. After three washing steps in PBS, coverslips were transferred to ICC blocking solution for $1 \mathrm{~h}$ at room temperature. Primary antibodies against MBP (myelinated segments) and TUJ1 (axons) were applied in blocking solution and incubated over night at $4{ }^{\circ} \mathrm{C}$. The next day, coverslips were washed again for three times in PBS before incubation in fluorescently labelled secondary antibodies and DAPI (nucleus) for 1 hour at room temperature. It followed another three washing steps in PBS and mounting in Mowiol. SC-DRG co-cultures were imaged using fluorescence microscopy and analyzed for number of myelinated axons and length of myelinated segments using FIJI.

\section{Primary Schwann cells}

For measurements of length to width ratio and focal adhesion visualization, primary Schwann cells were incubated in CSK buffer for 30 seconds at $4{ }^{\circ} \mathrm{C}$ before fixation in $4 \%$ PFA for 10 minutes. After permeabilization by $0.2 \%$ Triton for 10 minutes, cells were incubated in ICC blocking solution for $1 \mathrm{~h}$ at room temperature. Afterwards, cells were incubated in primary antibodies over night at $4^{\circ} \mathrm{C}$ followed by three more PBS washing steps. Secondary antibodies 
were applied in blocking solution together with DAPI for $1 \mathrm{~h}$ at room temperature. For Phalloidin staining cells were incubated in Phalloidin (1:100 in PBS) for $15 \mathrm{~min}$. Subsequently three more washing steps before cells were mounted in Mowiol mounting medium. Primary Schwann cells were imaged using fluorescence microscopy and analyzed for their width to length ratio using FIJI.

\section{Paraffin cross sections}

Nerves in Paraffin wax blocks were deparaffinated following the protocol below:

\begin{tabular}{l|l|l} 
Step & Duration & Temperature \\
Warm up & $10-20 \mathrm{~min}$ & $60{ }^{\circ} \mathrm{C}$ \\
Xylene & $10 \mathrm{~min}$ & RT \\
Xylene & $10 \mathrm{~min}$ & RT \\
$1: 1$ Xylene : 2-Propanol & $5 \mathrm{~min}$ & RT \\
$100 \%$ Ethanol & $5 \mathrm{~min}$ & RT \\
$90 \%$ Ethanol & $5 \mathrm{~min}$ & RT \\
$70 \%$ Ethanol & $5 \mathrm{~min}$ & RT \\
$50 \%$ Ethanol & $5 \mathrm{~min}$ & RT \\
ddH ${ }_{2} \mathrm{O}$ & $5 \mathrm{~min}$ & RT \\
Citrate buffer & $10-15 \mathrm{~min}$ & Boiling in microwave 600 watt \\
& $20 \mathrm{~min}$ & Cooling down \\
Tris buffered milk & $5 \mathrm{~min}$ & RT
\end{tabular}

Afterwards, slides were incubated in paraffin blocking solution for 20 minutes at room temperature to block unspecific binding sits. Primary antibody (in $0.5 \%$ BSA PBS) was incubated over night at $4{ }^{\circ} \mathrm{C}$. The next day, slides were washed with Tris buffered milk for three times before applying secondary antibodies and DAPI (in $0.5 \%$ BSA PBS) for 1 hour at room temperature. It followed another three washing steps in tris buffer and finally mounting in Mowiol mounting medium.

\section{Teased fibers}

Teased fibers were taken directly from $-20{ }^{\circ} \mathrm{C}$ and fixed in $4 \%$ PFA for five minutes and in ice cold Methanol/Acteon (95/5 [v/v]) for another five minutes. Furthermore, fibers were three times washed in PBS and incubated in teased fiber blocking solution for 1 hour at room temperature. After incubation with primary antibodies in blocking solution, three washing steps in PBS followed. Secondary antibodies and DAPI was diluted in blocking solution and fibers incubated for one hour at room temperature. Three more washing steps in PBS were performed and fibers mounted in Mowiol mounting medium. Teased fibers were imaged using fluorescence microscopy and analyzed for internodal length, fiber diameter and number of Schmidt-Lanterman Incisures using FIJI. 


\subsubsection{Gallays and Blue staining}

Semithin section of peripheral nerves were Gallays (Myelin) and Methylene blue/ Azur II (connective tissue, nuclei) stained for g-ratio quantifications as described in the following protocol:

\begin{tabular}{|c|c|c|}
\hline Step & Duration & Temperature \\
\hline Etching solution (Maxwell) & $5 \mathrm{~min}$ & RT \\
\hline Wash under running $\mathrm{ddH}_{2} \mathrm{O}$ & $10 \mathrm{~min}$ & RT \\
\hline Pyridine : Acetic acid anhydride (2:1) & $30 \mathrm{~min}$ & RT \\
\hline Wash in $\mathrm{ddH}_{2} \mathrm{O}$ & $10 \times 1 \mathrm{~min}$ & RT \\
\hline Incubation solution & $60 \mathrm{~s}$ & Microwave 150 Watt \\
\hline & $10 \mathrm{~min}$ & RT \\
\hline $0.5 \%$ Acetic Acid & $3 \times 5 \mathrm{~min}$ & RT \\
\hline Developing solution & $1 \mathrm{~min}$ & RT \\
\hline $1 \%$ Acetic Acid & $3 \times 5 \mathrm{~min}$ & RT \\
\hline Wash in $\mathrm{ddH}_{2} \mathrm{O}$ & $5 \mathrm{~min}$ & RT \\
\hline $2 \%$ sodium thiosulfate & $5 \mathrm{~min}$ & RT \\
\hline Wash in $\mathrm{ddH}_{2} \mathrm{O}$ & $5 \mathrm{~min}$ & RT \\
\hline Methylene blue/ Azur II & $1 \mathrm{~min}$ & RT \\
\hline Wash in $\mathrm{ddH}_{2} \mathrm{O}$ & $1 \mathrm{~min}$ & RT \\
\hline
\end{tabular}

After staining slides were mounted in Eukitt before imaging.

\subsubsection{Microscopy}

\section{Light Microscopy}

Semi-thin sections, stained with Methylene blue/ Azur II and Gallays, were imaged using the Image Z1 microscope (Zeiss). Tile images were acquired using the 100x oil immersion objective and stitched using the image software ZEN2.6 (blue edition). Images were exported as tif files and subjected for further data analysis.

\section{Fluorescence Microscopy}

Fluorescent images from teased fibers, paraffin cross-sections, SC-DRG co-cultures, primary Schwann cells as well as HEK cells were acquired using the Axiophot Observer Z (Zeiss) with a Colibri LED light source (Zeiss). Overview images were taken with the 10x air objective and high magnification images with the $40 x$ air or $63 x$ water immersion objective. For further image processing, SC-DRG co-cultures as well as teased fiber staining images were stitched and exported as tif using the ZEN2.6 (blue edition) software. 


\section{STED Microscopy}

Stimulated emission depletion microscopy (STED) was performed by Joris van Dort and Katrin Willig (Optical Nanoscopy in Neuroscience, Max Planck Institute of Experimental Medicine, Göttingen). A custom-build 3D two-color STED microscope was used (van Dort 2018) and nerve cross sections stained with secondary antibodies Starred and Alexa Fluor 594. Alexa Fluor 594 was excited at $586 \mathrm{~nm}$ and detected at $620 \mathrm{~nm}$, whereas Starred was excited at $650 \mathrm{~nm}$ and detected at $692 \mathrm{~nm}$. The STED emission of both dyes is at $775 \mathrm{~nm}$.

\subsection{Data analysis}

\subsubsection{Image Analysis}

Image analysis was conducted using FIJI (Fiji is just ImageJ) as previously described.

For g-ratio analysis, images of semithin sections were processed using the following FIJI protocol (Figure VIIa) to easily measure axon perimeter and myelin perimeter. Therefore, images were converted to binary images and using the particle analyzer allows automated recognition of axons (Figure VIIc) and myelin perimeters (Figure VIId), which can be

a
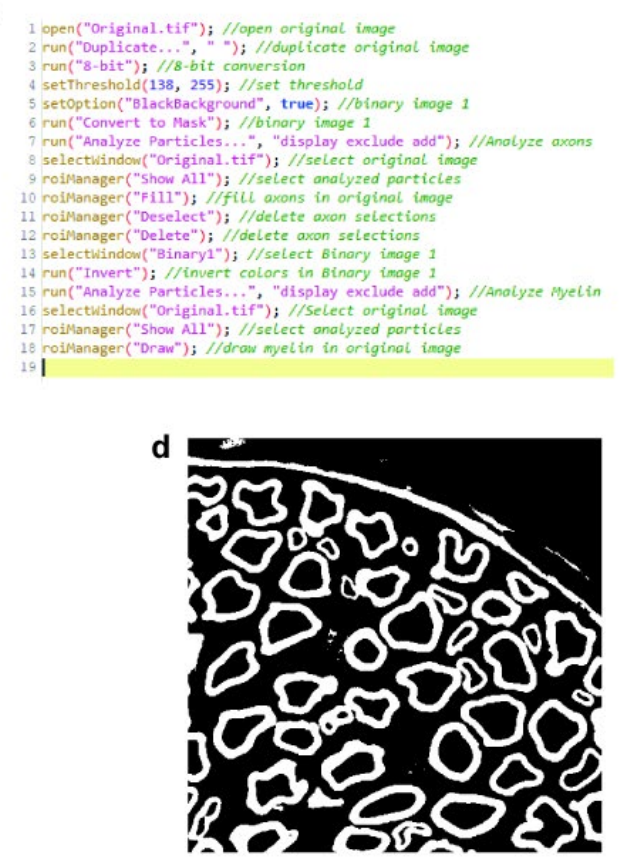

b

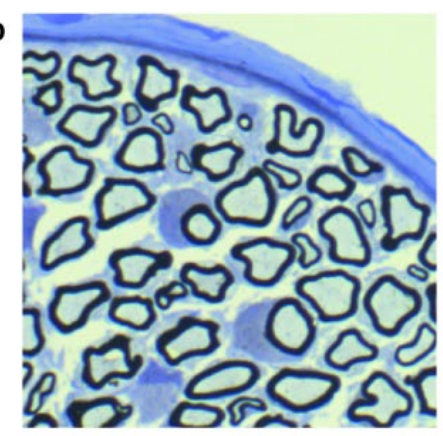

e

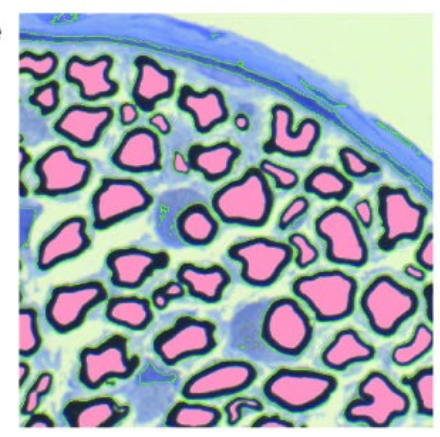

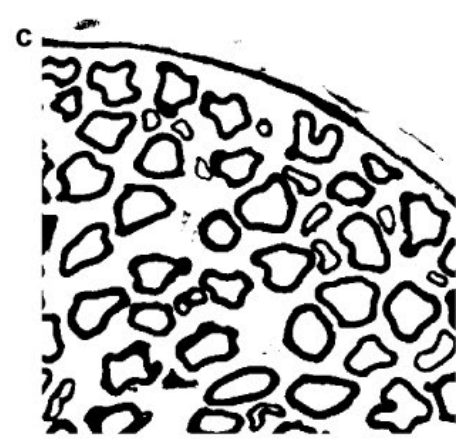

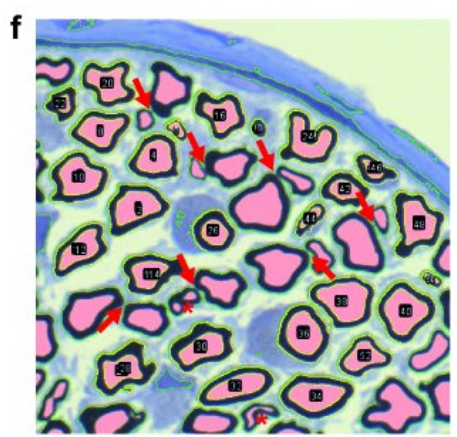

Figure VII: G-ratio analysis in whole femoral nerves using FIJI.

(a) Analysis steps in FIJI.

(b) Original image.

(c) Binary image 1 for axon analysis.

(d) Binary image 2 for myelin analysis.

(e) Analyzed axons are filled in pink and myelin perimeter is drawn in green.

(f) Wand (tracing) toll analysis allows quick assigning of axons and myelin sheaths. Arrows indicate not fully separated myelin sheaths and stars indicate inappropriately segmentation of axons. 
visualized in the original image (Figure VIIe). This allows easy selection of axons and the surrounding myelin sheath by using the 'wand (tracing) tool' in FIJI (Figure VIIf). The otherwise time-consuming circling of all axons is reduced, although segmentation is limited, as some axons are not recognized completely (Figure VIIf, stars) or myelin sheaths are not separated well enough (Figure VIIf, arrows). Therefore, the quantification is only semiautomated and not fully segmented axons and their myelin sheath are circled by hand. Nevertheless, this approach offers an improvement to the usually very time-consuming quantification by hand.

\subsubsection{Statistical analysis}

Data analysis was performed using Microsoft Excel 2016. The mean of all technical replicates was calculated and the mean of biological replicates was depicted as one data point. Standard deviation was calculated from the biological replicates and indicated as error bars $( \pm)$. Statistical analysis and diagram depiction was conducted using Graphpad Prism 8. Statistical tests are indicated in every figure. P-values under 0.05 are specifically indicated, significant values are depicted as following ${ }^{*} \mathrm{p} \leq 0.05,{ }^{* *} \mathrm{p} \leq 0.01,{ }^{* * *} \mathrm{p} \leq 0.001$ and ${ }^{* * * *} \mathrm{p} \leq 0.0001$. Figures were assembled in Adobe Illustrator 2020. 


\section{Results}

\subsection{ER stress as a disease mechanism in CMT1A?}

PMP22 is passing through secretory pathway from endoplasmic reticulum (ER) and Golgi apparatus to reach the plasma membrane (Pareek et al 1993). PMP22 point mutations have been associated with retained protein in the ER (D'Urso et al 1998, Naef et al 1997; D'Urso et al. 1998, Colby et al 2000, Tobler et al 1999). When misfolded proteins accumulate in the ER, they cause ER stress, activating the so-called unfolded protein response (UPR) (Walter and Ron 2011). In other demyelinating diseases, an upregulation of the UPR was observed and raised the question whether ER stress caused by PMP22 overexpression is a relevant disease mechanism in CMT1A and can be used as a pharmacological target (Lin and Popko 2009, D'Antonio et al 2009, Volpi et al 2016).

\subsubsection{CMT1A rats do not display ER stress.}

During ER stress, translation in the cell is inhibited to stop extra production of proteins whereas the transcription of chaperones and other cytoprotective genes is enhanced to handle the increased protein amount. A prolonged overactivation of the UPR leads to apoptosis and cell death (Walter and Ron 2011).

We analyzed the mRNA expression of typical ER stress markers gadd34, atf4, sXBP1, ddit3 and hspa5 in CMT1A tibial nerves at four different time points. We used the expression of the disease causing gene pmp22 and the lipid metabolism gene hmgcr as positive controls, which are up- and downregulated time-dependently in CMT1A rats (Fledrich et al 2014). In myelinating Schwann cells, lipid and protein production strongly increases during early development to reassure proper myelin sheath formation. We did not observe an upregulation of ER stress markers at postnatal day 18, reflecting a time point of high lipid and myelin protein production during development (Figure 1a). Moreover, young adult CMT1A rats at 9 weeks displayed no alteration in mRNA expression of UPR genes (Figure 1b). Only aged animals at 47 weeks of age showed a slight upregulation of gadd34 mRNA (Figure 1c), suggesting a potential secondary effect in CMT1A rats during aging (Paz Gavilan et al 2006). CMT1A rats show a very variable disease severity (Fledrich et al 2012a). Thus, we analyzed ER stress markers in mildly and severely affected rats. At 16 weeks of age neither mildly nor severely affected CMT1A rats displayed an upregulation of UPR genes (Figure 1d). 
$\stackrel{\infty}{a}$

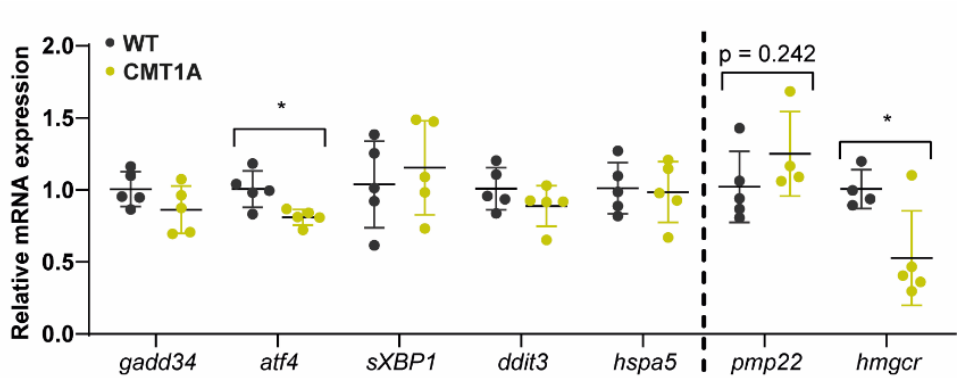

b
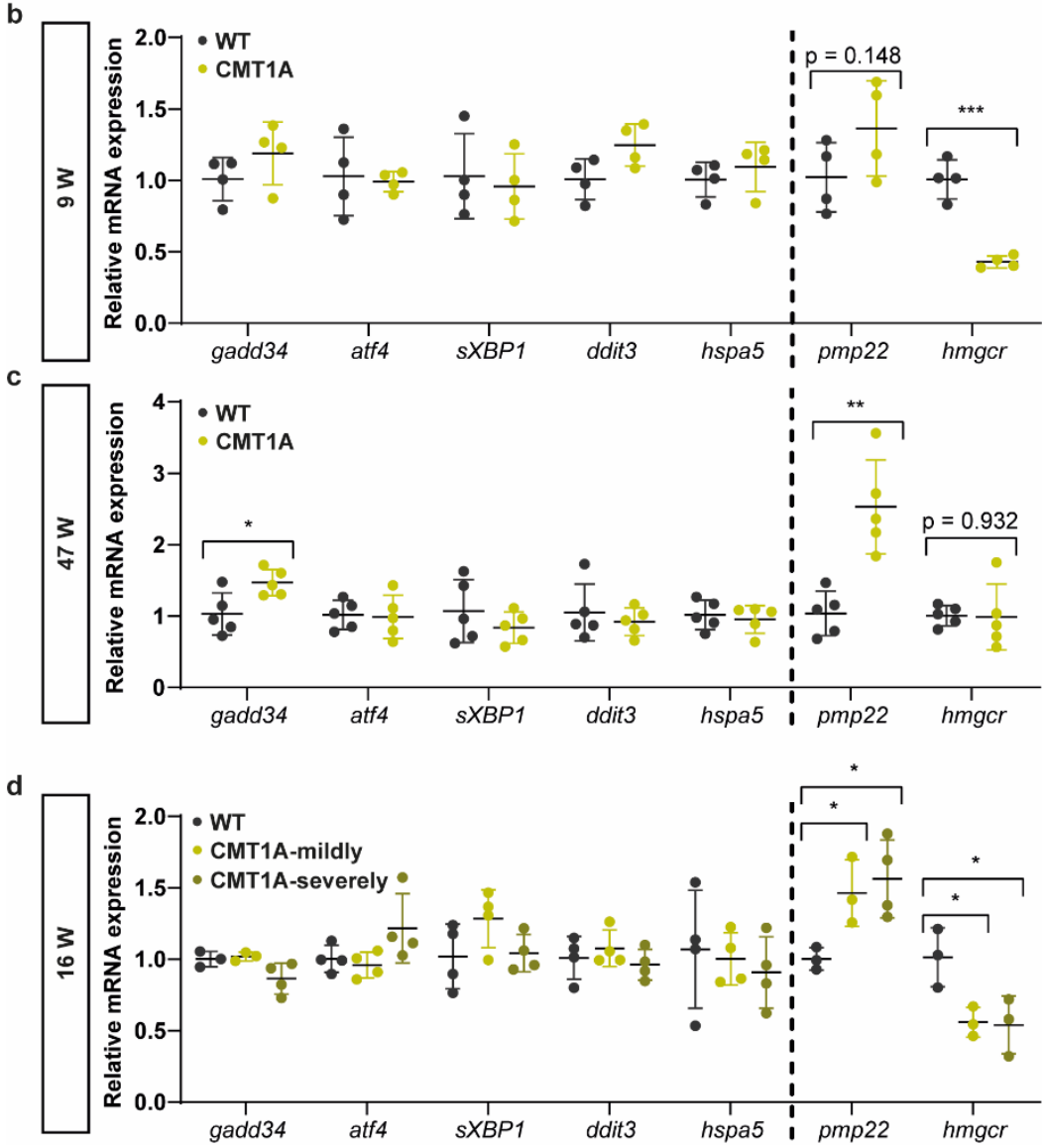

Figure 1: ER stress markers are unaltered in CMT1A rats.

(a) At postnatal day 18, CMT1A rat tibial nerves display indifferent expression of typical ER stress marker mRNA (gadd34, atf4, sXBP1, ddit3 and hspa5) compared to WT controls, as positive controls we used pmp22 and hmgcr mRNA expression.

(b) Moreover, at 9 weeks of age we observed unaltered mRNA expression of ER stress markers in CMT1A to WT.

(c) 47 weeks old CMT1A nerves show a significant upregulation of ER stress marker gadd34 while the other markers did not differ.

(d) Comparison of mildly and severely affected CMT1A rats at 16 weeks of age display no alteration in ER stress marker mRNA expression. (Robert Fledrich kindly provided RNA.)

Rplp0 and cycloA served as housekeeping genes. Statistical analysis: Multiple t-test (a-c), Repeated ANOVA with Tukey's multiple comparison test. ${ }^{*} \mathrm{p} \leq 0.05,{ }^{* *} \mathrm{p} \leq 0.01,{ }^{* * *} \mathrm{p} \leq 0.001$ and ${ }^{* * * *} \mathrm{p} \leq 0.0001$

In summary, CMT1A rats revealed no increase in UPR gene expression early in development and in adult mice, independent of disease severity. Only aged mice displayed a slight upregulation of gadd34. These results indicate that overexpressed PMP22 does not retain in the ER and reaches the plasma membrane early in development when the Schwann cell has to produce a high amount of lipids and proteins to facilitate proper myelination. Nevertheless, 
the observed upregulation of a single ER stress marker in older CMT1A rats points to ER stress as a secondary effect in CMT1A pathogenesis, which provoked us to test the effect of a drug targeting ER stress in SC-DRG co-cultures in vitro.

\subsubsection{Pharmacological prolongation of the unfolded protein response in CMT1A SC- DRG co-cultures does not improve myelination in vitro.}

Upon ER stress, translation in the cell is decreased by the phosphorylation of eIF2 $\alpha$. Sephin1 administration was shown to improve myelination in an animal model of CMT1B (Das et al 2015). Sephin1 inhibits GADD34, which acts as a negative feedback mechanism by dephosphorylating eIF $2 \alpha$ to reactivate translation. Prolonging the attenuation of translation by Sephin1 decreased the protein folding load in Schwann cells and improved myelination and thus providing a new therapeutical strategy for CMT1B.

a

WT

CMT1A
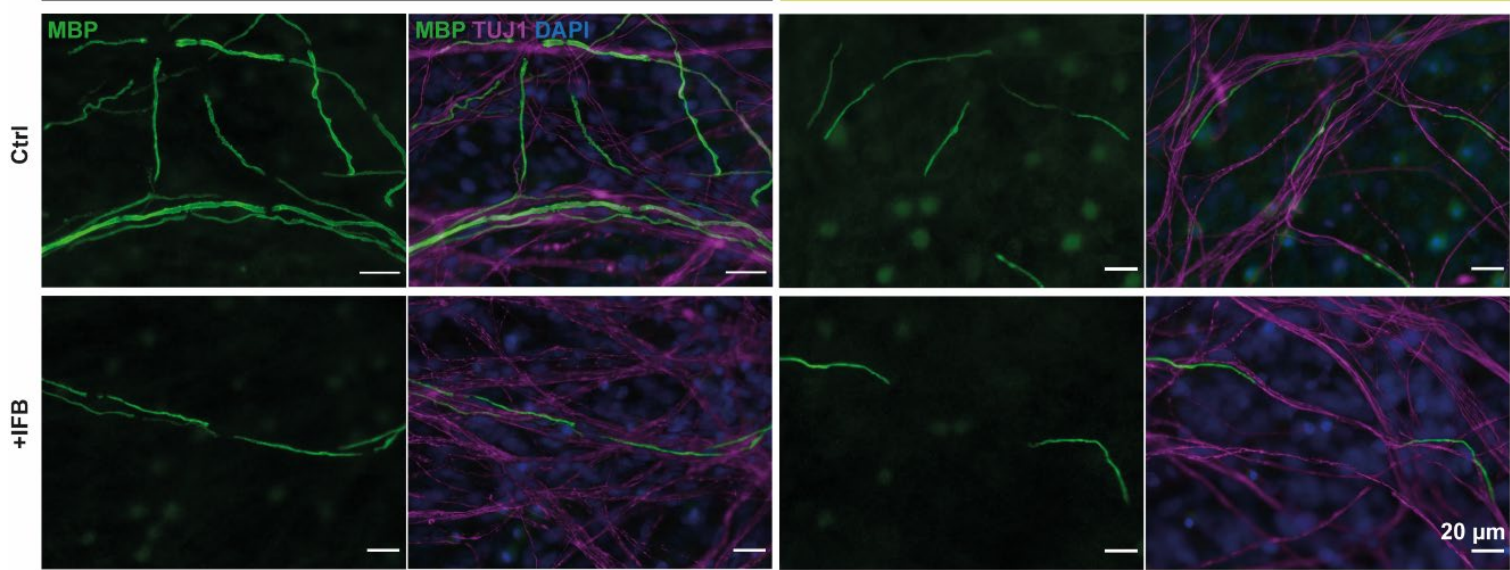

b

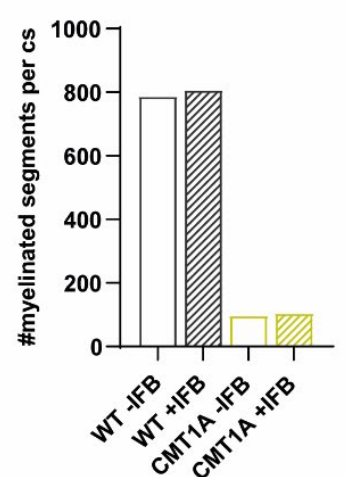

C $4-$ WT Ctrl
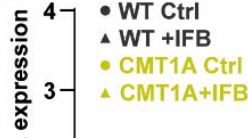

$\sum_{\substack{\alpha \\ \hline}}$
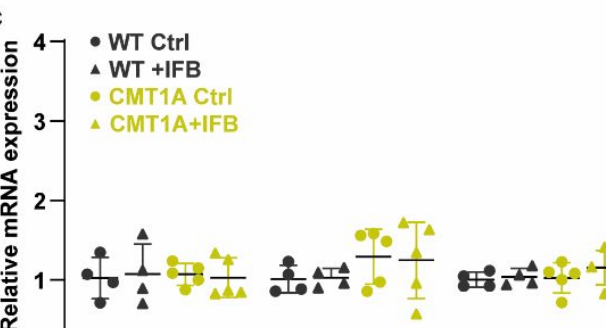

Figure 2: IFB-088 treatment in CMT1A SC-DRG co-cultures does not improve myelination.

(a) Example immunocytochemistry images of wildtype (left) and CMT1A (right) SC-DRG co-cultures without (upper panel) and with IFB treatment (lower panel). Myelin basic protein (green) indicates myelinated segments, TUJ1 visualizes axons (magenta) and DAPI cell nuclei (blue). Scale bar is $20 \mu \mathrm{m}$.

(b) CMT1A SC-DRG co-cultures (yellow) display reduced number of myelinated segments per coverslip, which does not change after IFB treatment (yellow, lines).

(c) Relative mRNA expression of ER stress marker gadd34, atf4 and hspa5 shows no alteration between genotypes and treatment groups. Positive control mRNA pmp22 shows a significantly increase in CMT1A co-cultures, independent from treatment.

Statistical Analysis: Repeated ANOVA with Tukey's multiple comparison test. ${ }^{*} \mathrm{p} \leq 0.05,{ }^{* *} \mathrm{p} \leq 0.01,{ }^{* * *} \mathrm{p} \leq 0.001$ and ${ }^{* * * *} \mathrm{p} \leq 0.0001$. 
We used IFB-088, also known as Sephin1, a compound from InFlectis BioScience, to treat SCDRG co-cultures from PMP22 overexpressing CMT1A rats in vitro. After two weeks of myelination in vitro, CMT1A co-cultures showed a reduced number of myelinated segments, which did not increase after IFB-088 treatment (Figure 2a,b). IFB-088 only works when the cell displays ER stress and GADD34 expression is upregulated. Similar to in the in vivo situation (Figure 1), SC-DRG co-cultures from CMT1A rats showed no upregulation of ER stress markers on mRNA level (Figure 2c). In line with this observation, IFB-088 treatment did not affect the expression of UPR genes in culture (Figure 2c).

The results indicate PMP22 overexpression in CMT1A does not cause ER stress as a primary defect early in myelin development. An upregulation of ER stress marker gadd34 in aged animals might be due to secondary effects and a sign of earlier aging in CMT1A (Paz Gavilan et al 2006). Treating SC-DRG co-cultures with IFB-088 did not improve myelination in vitro. Overall, we conclude that the pathomechanisms of CMT1A, which manifests already early in development, does not involve misfolding or intracellular aggregation of overexpressed PMP22. We hypothesize that overexpressed PMP22 reaches its destination, which enhances its largely unknown function and leads to the molecular and clinical phenotype in CMT1A. 


\subsection{Modulating PI3K/Akt/mTOR dependent growth signaling in PMP22 gene-dosage related diseases.}

In the previous section we demonstrated that PMP22 overexpression does not cause ER stress in CMT1A. These results encouraged us to further study the molecular function of PMP22 in more detail. PMP22 was originally discovered as Gas3 (growth arrest specific gene) in fibroblast (Schneider et al 1988). In 2014, Fledrich et al. reported on changes in the myelin growth signaling pathway PI3K/Akt/mTOR according to PMP22 gene-dosage. Heterozygous deletion of PMP22 increases and PMP22 overexpression decreases PI3K/Akt/mTOR signaling in Schwann cells (Fledrich et al 2014). The PI3K/Akt/mTOR pathway is tightly regulated in Schwann cell development to assure proper myelin growth (Domenech-Estevez et al 2016, Figlia et al 2017). CMT1A nerves display thinner myelin sheaths and reduced internodal length (Fledrich et al 2014, Saporta et al 2009), whereas in HNPP enhanced myelin growth leads to the formation of redundant myelin loops, so-called tomacula (Adlkofer et al 1995; Adlkofer et al 1997).

Hence, we hypothesize that PMP22 gene dosage acts as a break on myelination, mediated by the PI3K/Akt/mTOR signaling pathway. Therefore, we further investigated and modulated the effects of misbalanced growth signaling in animal models of PMP22 gene-dosage dependent diseases. As CMT1A rodent models we used the CMT1A rat (Sereda et al 1996), which closely recapitulates the phenotype in patients, and the C61 mouse model of CMT1A (Huxley et al 1998), which is slightly less affected compared to CMT1A rats. Moreover, we used PMP22 heterozygous knockout mice, as they provide a rodent model for HNPP (Adlkofer et al 1995).

\subsubsection{PMP22 dosage perturbs the homeostasis of the major growth inhibitor PTEN in rodent disease models of CMT1A and HNPP.}

The major inhibitor of the PI3K/Akt/mTOR pathway is Phosphatase and tensin homolog (PTEN), which counteracts PI3Kby dephosphorylating PIP 3 to $\mathrm{PIP}_{2}$, resulting in a downstream inhibition of Akt. Ablation of PTEN in Schwann cells results in an overactivation of the PI3K/Akt/mTOR pathway and hypermyelination of axons (Goebbels et al 2012, Figlia et al 2017), a phenotype similar to HNPP.

Western Blot analysis revealed an increase of PTEN protein in CMT1A which is vice versa and a decrease in HNPP (Figure 3a,b, Figure $\mathbf{4 a} \mathbf{a}, \mathbf{b}$ ). In CMT1A nerves, the increase in PTEN protein abundance is consistent from postnatal day 6 until 9 weeks of age. Although, at 9 weeks of age PTEN showed a less prominent abundance compared to the early developmental stages at P6 and P18 (Figure 3a,b), suggesting a more prominent role for PTEN early in development. Moreover, on transcriptional level pten mRNA was also pmp22 dependently changed in animals models of CMT1A and HNPP (Figure 3c, Figure 4c). In peripheral nerves, PTEN localized to the axon, Schwann cell nuclei and cytoplasmic non-compact compartments such 
a

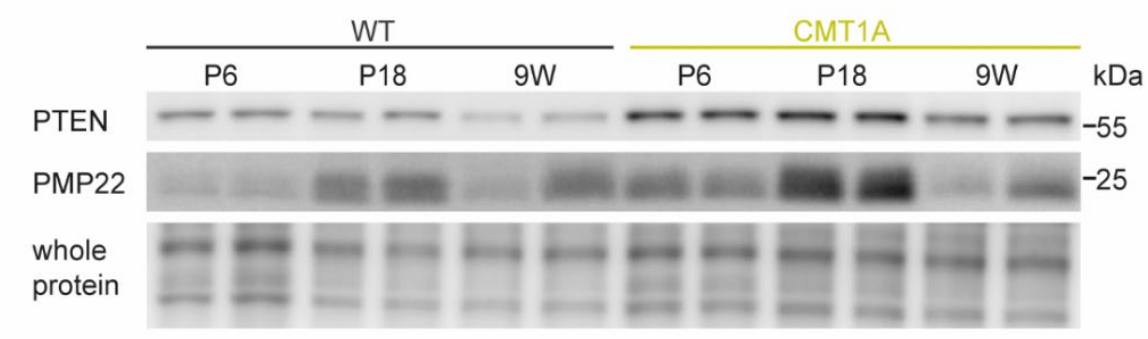

b
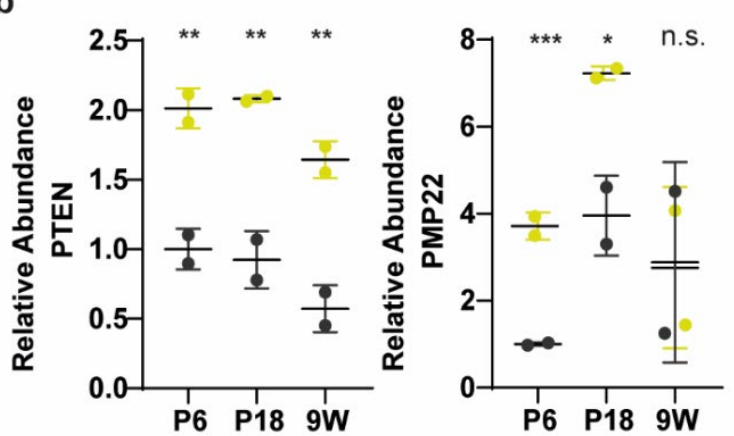

C

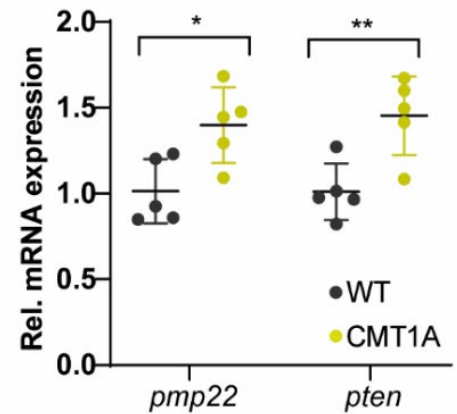

Figure 3: Increase of PTEN on protein and mRNA level in a rat model of CMT1A.

(a) Immunoblot analysis of PTEN and PMP22 in sciatic nerve lysates from CMT1A rats and wildtype controls at postnatal day 6, 18 and 9 weeks. Fast green whole protein staining as loading control.

(b) Quantification of (a), revealing an increase of PTEN and PMP22 protein levels in CMT1A (yellow) compared to wildtype controls (grey).

(c) qRT-PCR analysis of pmp22 and pten mRNA from CMT1A (yellow) and wildtype control (grey) p 18 tibial nerves showing an upregulation of pmp22 and pten mRNA in CMT1A.

Statistical analysis: (b) Multiple t-test, (c) unpaired student's t-test, mean $\pm \mathrm{SD},{ }^{*} \mathrm{p} \leq 0.05,{ }^{* *} \mathrm{p} \leq 0.01,{ }^{* * *} \mathrm{p} \leq 0.001$, **** $\mathrm{p} \leq 0.0001$.

as bands of Cajal (Figure 5). In nerve cross section from CMT1A rats, PTEN localization did not change dramatically (Figure 5), indicating the overproduced PTEN reached its correct localization in the Schwann cell.

The finding of concordant alteration of PTEN with pmp22 gene-dosage suggests that the alteration of the PI3K/AKT/mTOR pathway in the opposite direction in both CMT1A and HNPP is mediated by PTEN as the main inhibitor of the pathway. The observations raise the questions whether targeting PTEN and the downstream signaling pathway pharmacologically can be used as a therapeutic target and how PMP22 influences PTEN amounts in the Schwann cell. 
a

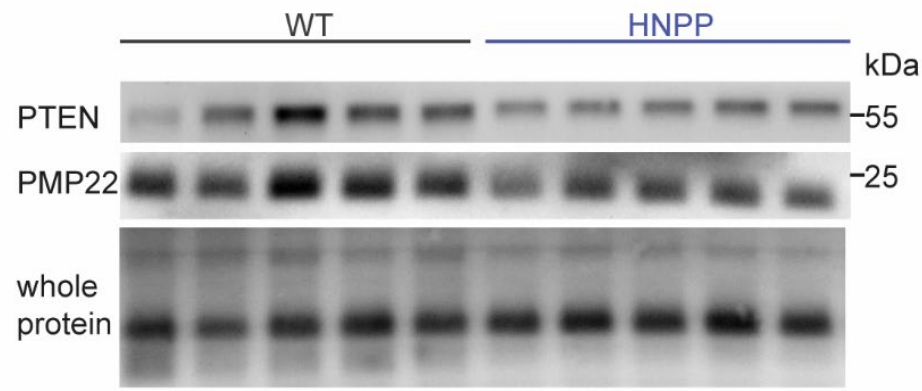

b

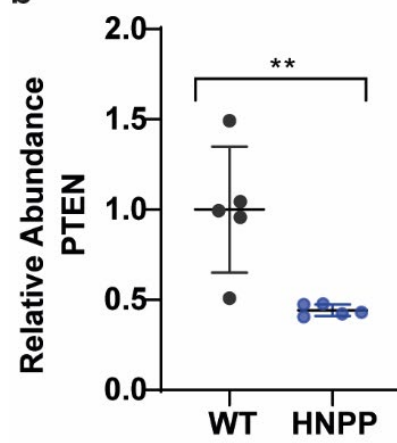

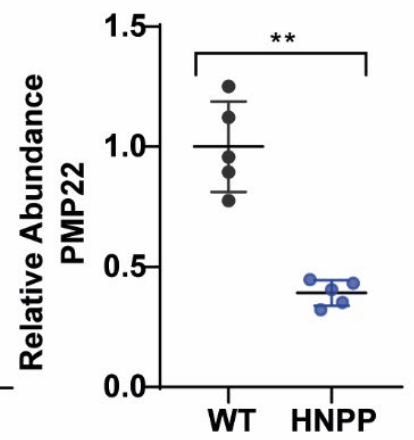

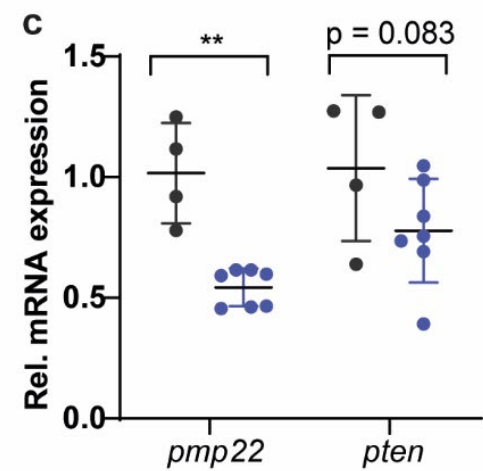

Figure 4: PTEN is decreased on protein and mRNA level in a mouse model of HNPP.

(a) Immunoblot analysis of PTEN and PMP22 in sciatic nerve lysates from HNPP mice and wildtype controls at 9 weeks. Fast green whole protein staining as loading control.

(b) Quantification of (a), revealing a decrease of PTEN and PMP22 protein levels in HNPP (blue) compared to wildtype controls (grey).

(c) qRT-PCR analysis of pmp22 and pten mRNA from HNPP (blue) and wildtype control (grey) p 18 tibial nerves showing a downregulation of pmp22 and pten mRNA in HNPP.

Statistical analysis: (c) unpaired student's t-test, mean $\pm \mathrm{SD},{ }^{*} \mathrm{p} \leq 0.05,{ }^{* *} \mathrm{p} \leq 0.01,{ }^{* *} \mathrm{p} \leq 0.001,{ }^{* * *} \mathrm{p} \leq 0.0001$.
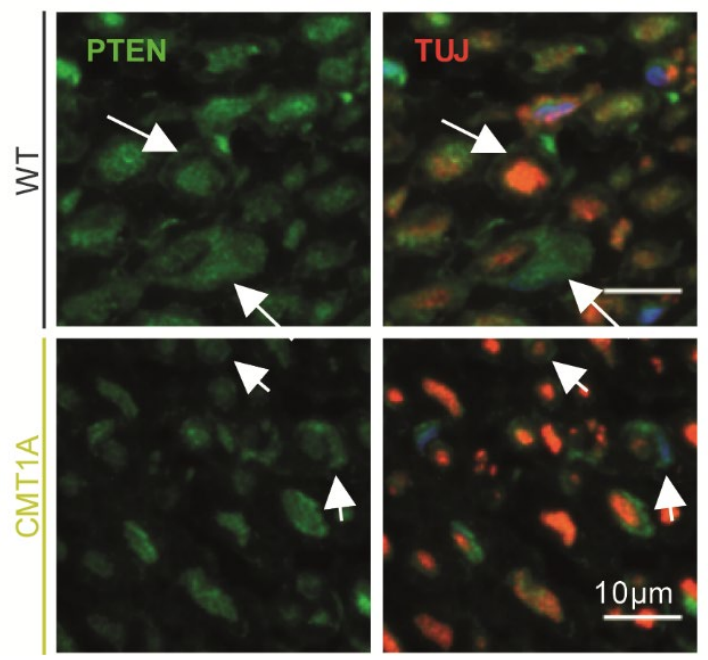

Figure 5: PTEN localization is unchanged in CMT1A.

Femoral nerve cross section of 9 weeks old wildtype (upper row) and CMT1A rats (lower row) show PTEN (green) localization to the axon, nuclei and bands of Cajal (arrows). 


\subsubsection{Counteracting dysregulated PI3K/Akt/mTOR signaling is beneficial in an animal model of HNPP.}

\section{Rapamycin treatment decreases outfoldings in HNPP in vitro.}

To counteract the overactivation of the PI3K/Akt/mTOR pathway in HNPP mice, we treated SC-DRG co-cultures in vitro with $20 \mathrm{nM}$ Rapamycin, an antibiotic specifically inhibiting mTOR downstream of PTEN and Akt.

Immunocytochemical analysis revealed a strong increase of myelin segments with outfoldings in SC-DRG co-cultures from HNPP mice compared to wildtype controls (Figure 6a,b). Outfoldings in cultures are observed at paranodal regions and can be compared to tomacula formation in vivo, a clinical hallmark of the HNPP disease. Treatment with Rapamycin for two weeks significantly decreased segments with outfoldings in HNPP cultures (Figure 6b), while the number of myelinated segments, which was not significantly altered in HNPP, remained unchanged (Figure 6c). Moreover, HNPP segments appeared slightly shorter than wildtype controls, and Rapamycin treatment did not affect segment length (Figure 6d).

To sum up, inhibiting mTOR with Rapamycin in HNPP SC-DRG co-cultures reduced aberrant myelin growth in vitro.

a
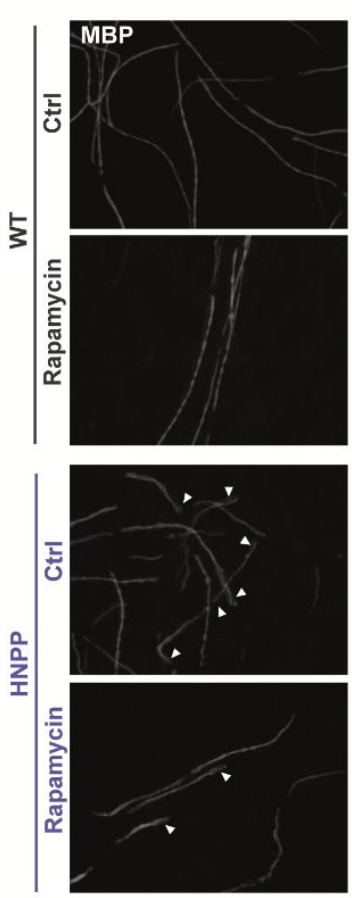
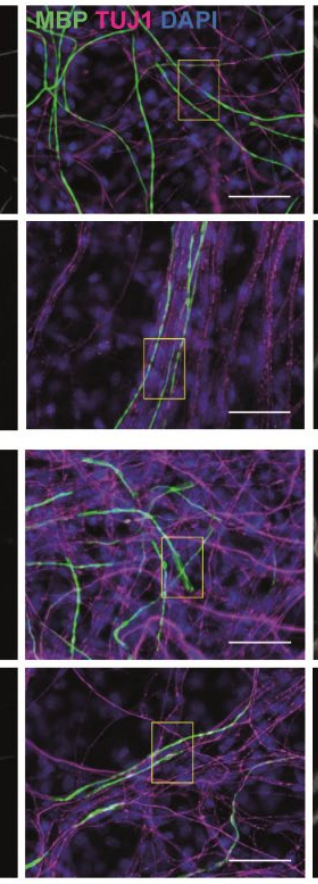

b
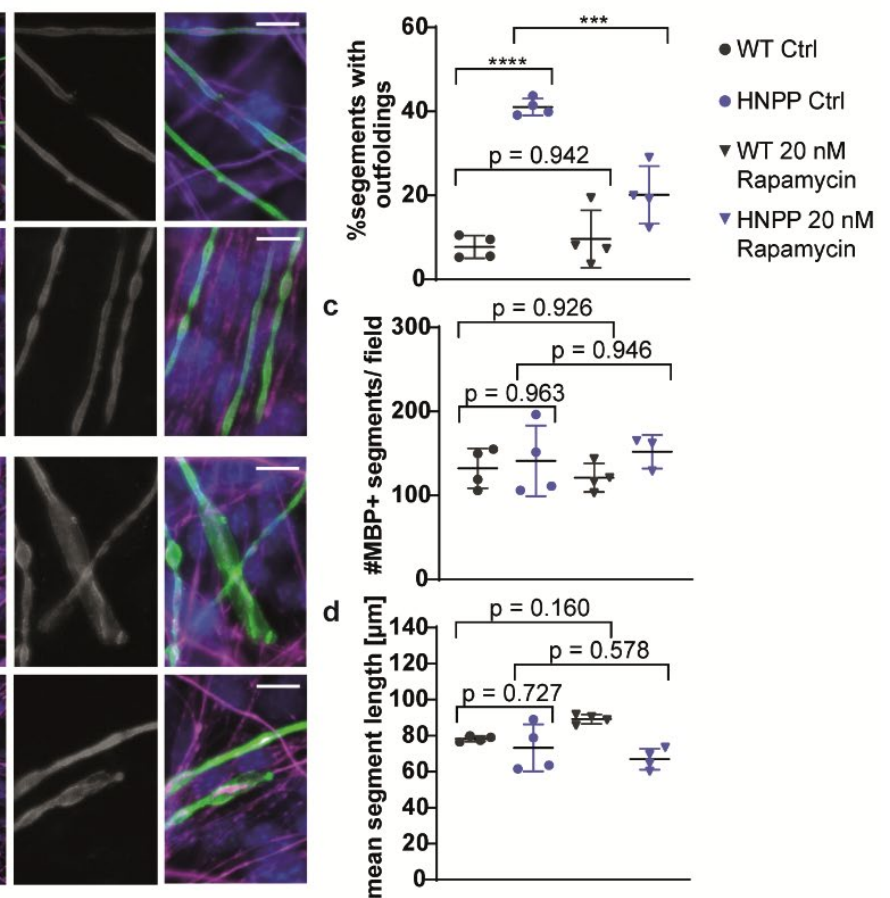

Figure 6: Inhibition of mTOR using Rapamycin decreases the number of myelin outfoldings in an HNPP animal model in vitro.

- $\quad$ Figure legend continues next page - 
(a) Example images of SC-DRG co-cultures after 14 days of myelination in vitro. The first (overview) and third (blow-up) column show MBP positive myelinated segments (white). The second (overview) and fourth (blowup) column depict the overlay of MBP (myelin, green), TUJ1 (neurons, magenta) and DAPI (nuclei, blue). The first row of images displays the wildtype control cultures and the second the Rapamycin treated wildtype. In the third row the HNPP control and in the fourth row the Rapamycin treated HNPP cultures are depicted, white arrowheads indicating myelin outfoldings. Scale bar in the overview images is $50 \mu \mathrm{m}$, in the blow-up images $10 \mu \mathrm{m}$.

(b) Quantification of the percentage of myelinated segments with outfoldings revealing an increase of outfoldings in untreated HNPP cultures (blue circle) compared to the wildtype (grey circle) and a reduction of outfoldings in HNPP cultures after treatment (blue triangle).

(c) Quantification of MBP positive segments per field is unchanged between genotypes and independent of Rapamycin treatment.

(d) Quantification of the length of MBP positive segments.

$\mathrm{n}=4$ biological replicates (embryos) per genotype and condition, with $\mathrm{n}=5$ technical replicates $(500 \times 500 \mu \mathrm{m}$ fields) were quantified. Biological means are displayed \pm SD. Statistical analysis: One-way ANOVA with Sidak's multiple comparison test. ${ }^{*} \mathrm{p} \leq 0.05,{ }^{* *} \mathrm{p} \leq 0.01,{ }^{* * *} \mathrm{p} \leq 0.001$ and ${ }^{* * *} \mathrm{p} \leq 0.0001$.

\section{Rapamycin treatment decreases hallmarks of HNPP phenotype in vivo}

Sandra Göbbels and colleagues translated the in vitro findings in vivo and found an overall improvement of the HNPP phenotype (Figure 7). HNPP and control mice were treated early from postnatal day 21 until postnatal day 148 with Rapamycin (Figure 7a). WT and HNPP Rapamycin-treated cohorts gained less weight compared to their respective Placebo groups (Figure 7b), as inhibiting a major growth pathway during development also influences the general growth of the animals. Although they gained less weight, the forelimb grip strength in Rapamycin treated WT animals was not affected (Figure 7c), suggesting no effect of Rapamycin on the normal wildtype motor behavior. Counteracting the PI3K/Akt/mTOR imbalance by Rapamycin administration in HNPP mice increased the forelimb grip strength, though they did not reach WT levels (Figure 7c). Electrophysiological measurements revealed lower CMAP amplitudes and slower nerve conduction velocities in HNPP mice. After Rapamycin treatment, amplitudes increased, while conduction velocity did not alter (Figure

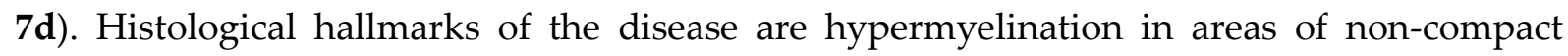
myelin forming tomacula and recurrent myelin loops. Quantification of tomacula and recurrent loops in cross sections of sciatic nerves from Rapamycin treated animals revealed a significant decrease in both structures (Figure 7e,f), which can explain the improvements in the phenotype and electrophysiology of Rapamycin treated HNPP mice.

On the molecular level, the relative abundances of PTEN were restored in HNPP mice that were treated with Rapamycin (Figure 8). As the inhibitor acts on mTOR downstream of PTEN, these results indicate a feedback mechanism from the downstream signaling components to PTEN. In summary, counteracting the increased activity in the PI3K/Akt/mTOR pathway in HNPP mice improves myelination in vitro and in vivo. 
a

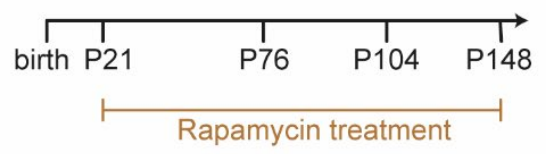

c

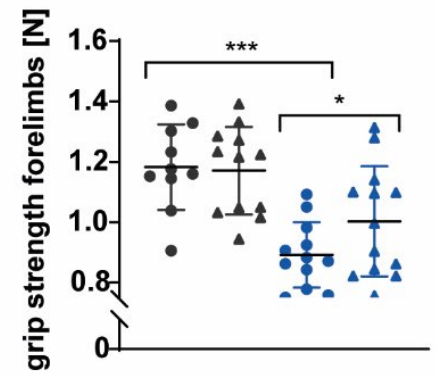

e

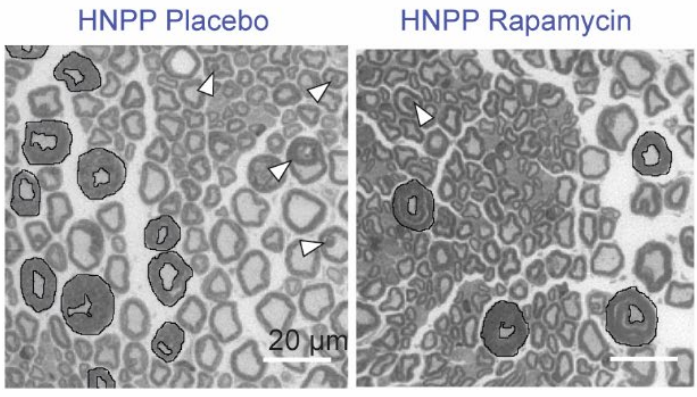

b

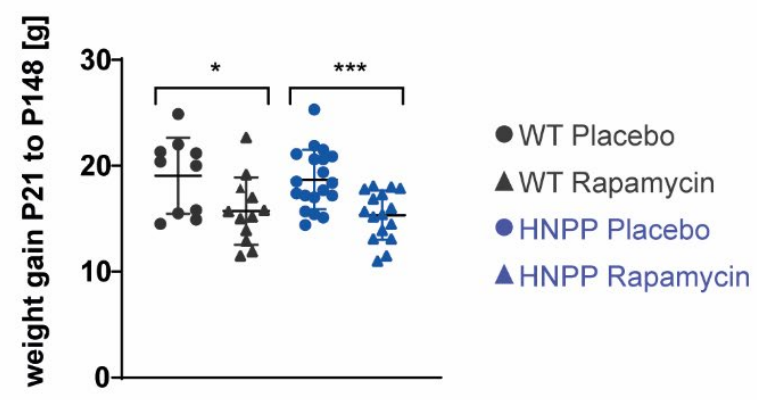

d
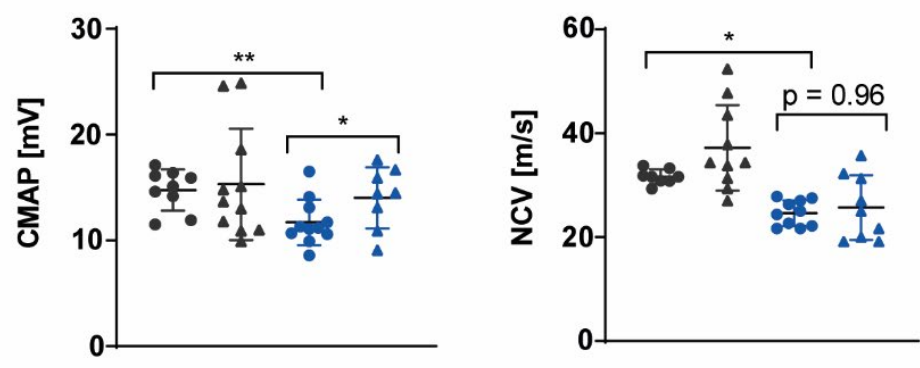

f

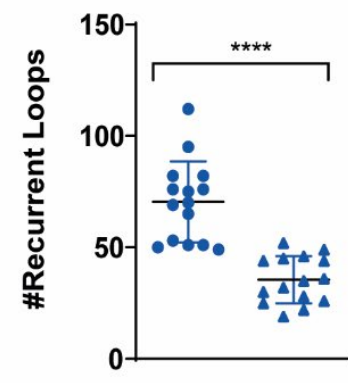

Figure 7: Inhibiting mTOR in an animal model of HNPP improves the phenotype in vivo.

(a) HNPP treatment paradigm, mice were i.p. injected with $5 \mathrm{mg}^{*} \mathrm{~kg}^{-1 *} \mathrm{~d}^{-1}$ Rapamycin two times per weeks from $\mathrm{p}$ 21 to $\mathrm{p} 148$.

(b) Weight gain of Rapamycin-treated (triangle) animals is reduced in comparison to placebo (circle).

(c) HNPP placebo mice (circle, blue) display decreased forelimb grip strength compared to the wildtype placebo mice (circle, grey) and improves after Rapamycin treatment (triangle, blue).

(d) Electrophysiological measurement reveal a decrease of CMAP and NCV in HNPP placebo mice to the wildtype placebo. After Rapamycin treatment CMAP is improved in HNPP (left panel) while NCV is unchanged (right panel).

(e) Example images of HNPP sciatic nerve cross sections. Left panel shows an HNPP placebo nerve and the right panel a Rapamycin treated nerve, Tomacula are circled in black and white arrowheads indicate recurrent loops. Scale bar is $20 \mu \mathrm{m}$.

(f) Quantification of (e) shows a decrease in the number of Tomacula (left panel) and Recurrent Loops in HNPP Rapamycin treated nerves (triangle, blue) compared to the placebo group (circle, blue).

Means are displayed \pm SD. Statistical analysis: One-way ANOVA with Sidak's multiple comparison test $(b, c, d, g)$ and unpaired student's t-test (f). ${ }^{*} \mathrm{p} \leq 0.05,{ }^{* *} \mathrm{p} \leq 0.01,{ }^{* * *} \mathrm{p} \leq 0.001$ and ${ }^{* * * *} \mathrm{p} \leq 0.0001$.

Stefan Volkmann and Sandra Göbbels performed Rapamycin treatment, behavior analysis and histology, Robert Fledrich performed Electrophysiology. 

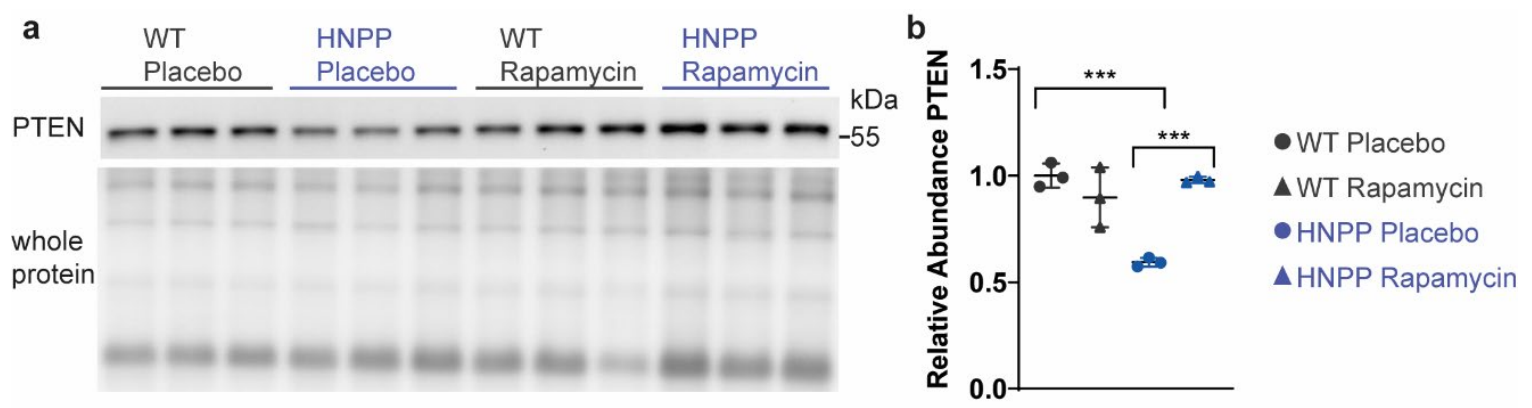

Figure 8: PTEN protein levels are restored in HNPP mice after Rapamycin treatment.

(g) Immunoblot analysis of PTEN in whole sciatic nerve lysates from (left to right) WT Placebo, HNPP Placebo, WT Rapamycin and HNPP Rapamycin animals at P148. Fast green whole protein staining is used as loading control.

(h) Quantification of (a) reveals restored PTEN protein levels in HNPP mice after Rapamycin treatment.

Means are displayed \pm SD. Statistical analysis: One-way ANOVA with Sidak's multiple comparison test. * $\mathrm{p} \leq 0.05$, ${ }^{* *} \mathrm{p} \leq 0.01,{ }^{* * *} \mathrm{p} \leq 0.001$ and ${ }^{* * * *} \mathrm{p} \leq 0.0001$.

\subsubsection{Pharmacologically increasing PI3K/Akt/mTOR pathway activity in CMT1A improves myelination in vitro.}

Counteracting the upregulated PI3K/Akt/mTOR pathway in HNPP mice showed reduced aberrant myelin and improved the HNPP phenotype in vivo. In CMT1A, the PI3K/Akt/mTOR pathway is downregulated. We aimed at increasing the pathway's activity by targeting the inhibitor PTEN in SC-DRG co-cultures in vitro.

\section{Inhibition of PTEN improves myelination in CMT1A in vitro.}

In CMT1A, PTEN is upregulated resulting in a stronger inhibition of the PI3K/Akt/mTOR pathway (Figure 3, [Fledrich et al 2014]). Rosivatz et al 2006 developed a small vanadium complex (VO-OHpic) which is a highly potent and specific inhibitor of PTEN, leading to increased PtdIns $(3,4,5) \mathrm{P} 3$ concentrations and Akt phosphorylation. In in vitro SC-DRG cocultures of CMT1A rats, the number of myelinated segments is strongly decreased (Figure $\mathbf{9 a , b}$ ). Upon VO-OHpic treatment, WT co-cultures displayed a dosage-dependent reduction in myelinated segments (Figure 9b). Importantly, in CMT1A the number of myelinated segments appeared slightly improved with $50 \mathrm{nM}$ and was significantly enhanced with $500 \mathrm{nM}$ VOOHpic. An even stronger inhibition of PTEN using $5 \mu \mathrm{M}$ VO-OHpic diminished myelination in CMT1A similar to the wildtype situation (Figure 9a-c). The length of myelin segments appeared shorter in CMT1A co-cultures and slightly longer after inhibition with $500 \mathrm{nM}$ VOOHpic.

Thus, although PTEN inhibition with $500 \mathrm{nM}$ VO-OHpic did not reach wildtype myelination, tuning down the PTEN activity is beneficial for myelination in CMT1A in vitro. Interestingly, PTEN inhibition dosage-dependently decreased myelination in WT cultures. PTEN is expressed in axons as well and therefore in the SC-DRG co-culture system a possible negative effect of axonal PTEN inhibition on myelination cannot be ruled out. 

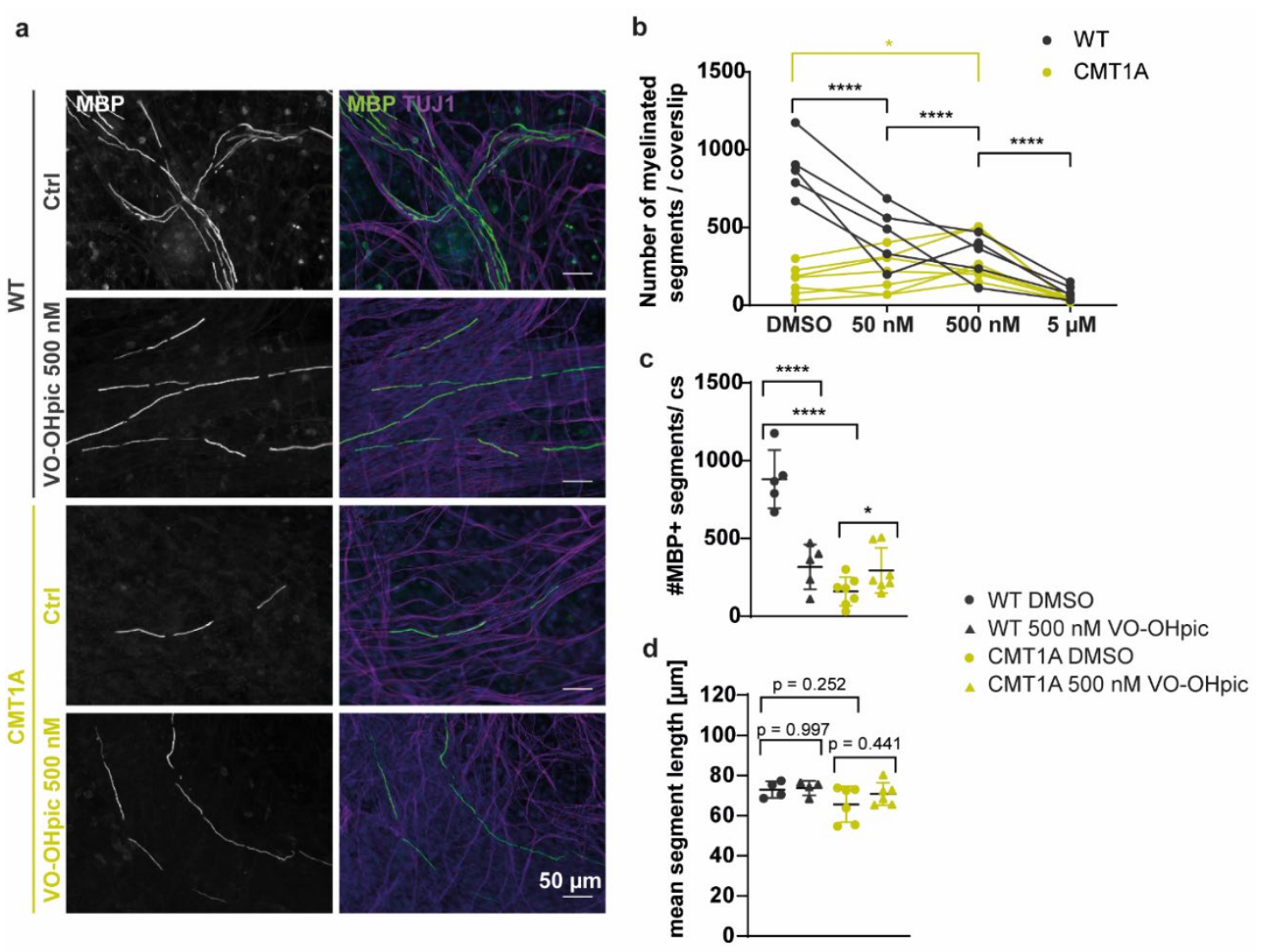

Figure 9: Inhibiting PTEN increases myelination in CMT1A SC-DRG co-cultures in vitro.

(a) Example images of SC-DRG co-cultures from wildtype (grey) and CMT1A rat embryos (yellow) after 14 days of myelination in vitro. The first column shows MBP positive myelinated segments (white), the second column depicts the overlay of MBP (myelin, green) and TUJ1 (neurons, magenta). The first row of images displays the wildtype control cultures and the second the $500 \mathrm{nM} \mathrm{VO-OHpic} \mathrm{treated} \mathrm{wildtype} \mathrm{cultures.} \mathrm{In} \mathrm{the} \mathrm{third} \mathrm{row} \mathrm{the}$ CMT1A control and in the fourth row the $500 \mathrm{nM}$ VO-OHpic treated CMT1A cultures are depicted. Scale bar is $50 \mu \mathrm{m}$.

(b) Dosage-dependent quantification of the number of myelinated segments per coverslip. In wildtype (grey) SCDRG co-cultures VO-OHpic dosage dependently decreases the number of myelinated segments while in CMT1A cultures (yellow) the number of myelinated segments is slightly increased with $500 \mathrm{nM}$ VO-OHpic and decreased at $5 \mu \mathrm{M}$.

(c) The number of myelinated segments is reduced in DMSO CMT1A cultures (circle, yellow) compared to the DMSO wildtype controls (circle, grey). $500 \mathrm{nM}$ VO-OHpic reduce the number of myelinated segments in the wildtype (triangle, grey) and increase the number in CMT1A cultures (triangle, yellow).

(d) Segment length tends to be shorter in CMT1A control cultures (circle, yellow) and slightly longer after VOOHpic treatment (triangle, yellow).

$\mathrm{n}=4-6$ biological replicates (embryos) per genotype and condition. Means are displayed \pm SD. Statistical analysis: One-way ANOVA with Sidak's multiple comparison test. ${ }^{*} \mathrm{p} \leq 0.05,{ }^{* *} \mathrm{p} \leq 0.01,{ }^{* * *} \mathrm{p} \leq 0.001$ and ${ }^{* * * *} \mathrm{p} \leq 0.0001$.

\subsubsection{Genetic reduction of PTEN in CMT1A increases myelination in vitro and early in vivo.}

\section{Generation of a Schwann cell specific heterozygous PTEN knockout in CMT1A Schwann cells.}

Given the strong effects of the PTEN inhibitor also on WT cultures, we further used a genetic approach instead of VO-OHpic to study the effects of PTEN reduction specifically in CMT1A Schwann cells. CMT1A mice were crossbred with PTEN heterozygous floxed mice under the 


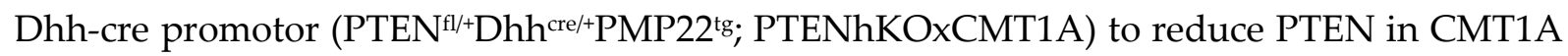
Schwann cells without effecting axonal PTEN (Figure 10a). Western Blot analysis revealed a reduction of PTEN in the double mutants and a corresponding activation of Ribosomal protein S6 downstream of AKT and mTOR (Figure 10b-d).

Furthermore, we investigated the effects of PTEN reduction in CMT1A mice (i) in vitro in SCDRG co-cultures and (ii) in vivo by analyzing histological, phenotypical and electrophysiological factors.

a

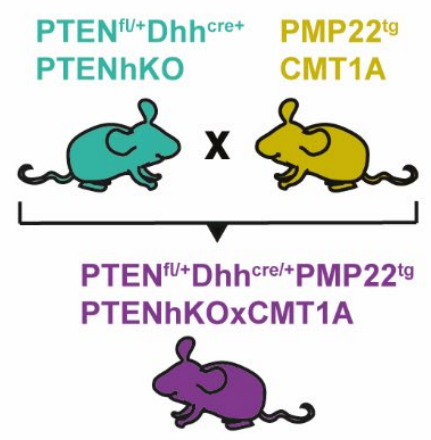

b

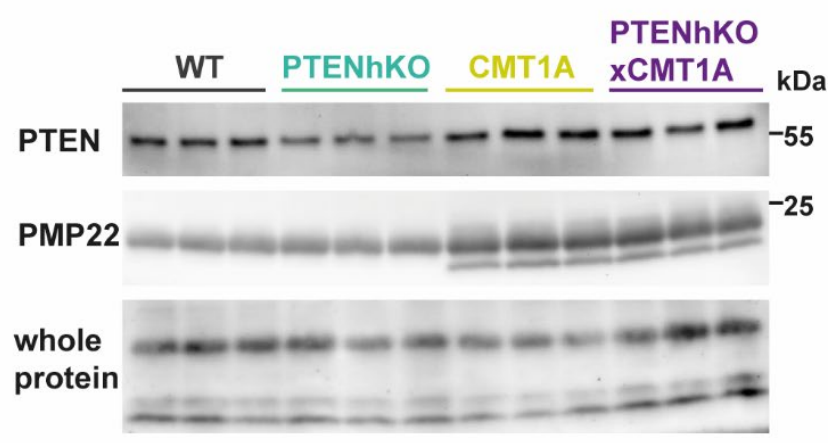

d

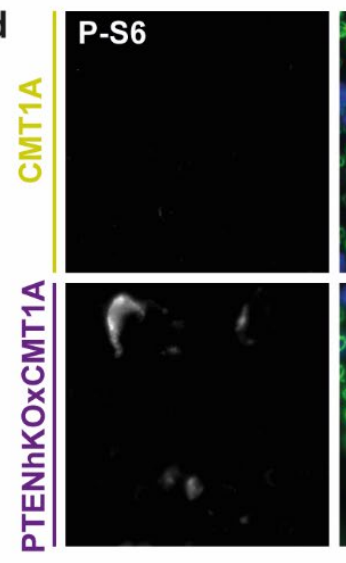

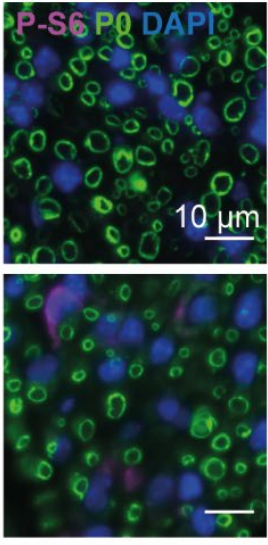

C

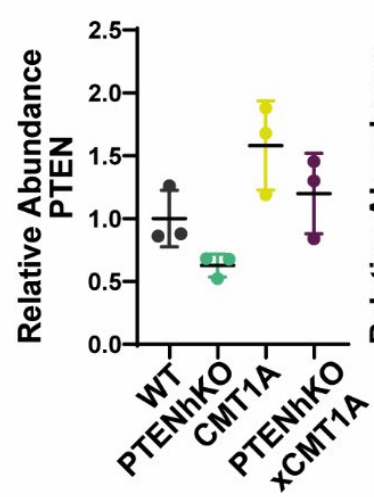

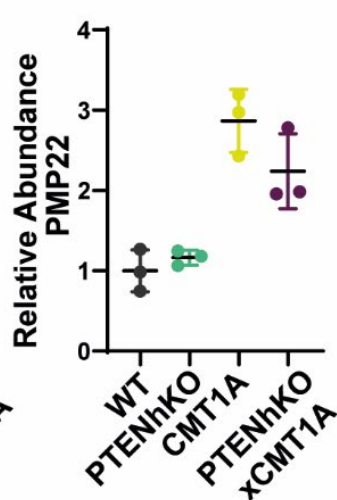

Figure 10: Genetic reduction of PTEN levels in CMT1A in vivo.

(a) Crossing scheme of PTEN SC specific heterozygous knockout mice (PTENhKO, turquoise) with PMP22tg mice (CMT1A, yellow) to generate double mutants (PTENhKOxCMT1A, purple).

(b) Immunoblot analysis of PTEN and PMP22 in sciatic nerve lysates from 16 weeks old WT (grey), PTENhKO (turquoise), CMT1A (yellow) and PTENhKOxCMT1A (purple) mice. Fast green whole protein staining as loading control.

(c) Quantification of relative PTEN (left panel) and PMP22 protein level (right panel).

(d) Immunohistochemistry of CMT1A and PTENhKOxCMT1A femoral nerve cross sections reveal increased Phospho-S6 (left: white, right: magenta) in the nuclei (DAPI, blue) of the double mutants (lower panel). P0 (green) is used as a marker for compact myelin. Scale bar is $10 \mu \mathrm{m}$.

\section{Heterozygous deletion of PTEN in CMT1A Schwann cells enhances myelination in vitro.}

SC-DRG co-cultures of the PTENhKOxCMT1A mice displayed improved myelination in vitro. The number of myelinated segments significantly increased in the double mutants compared to CMT1A mice and the segments appeared longer (Figure 11a-c). In contrast to the use of a PTEN inhibitor, this genetic approach of a heterozygous PTEN reduction in Schwann cells 
alone (PTENhKO), did not have an impact on the number and length of myelinated segments in vitro (Figure 11), indicating the negative correlation of the PTEN inhibitor might indeed be due to an effect on axonal PTEN.

These results show that reducing Schwann cell PTEN in an animal model of CMT1A improves myelination in vitro.

a
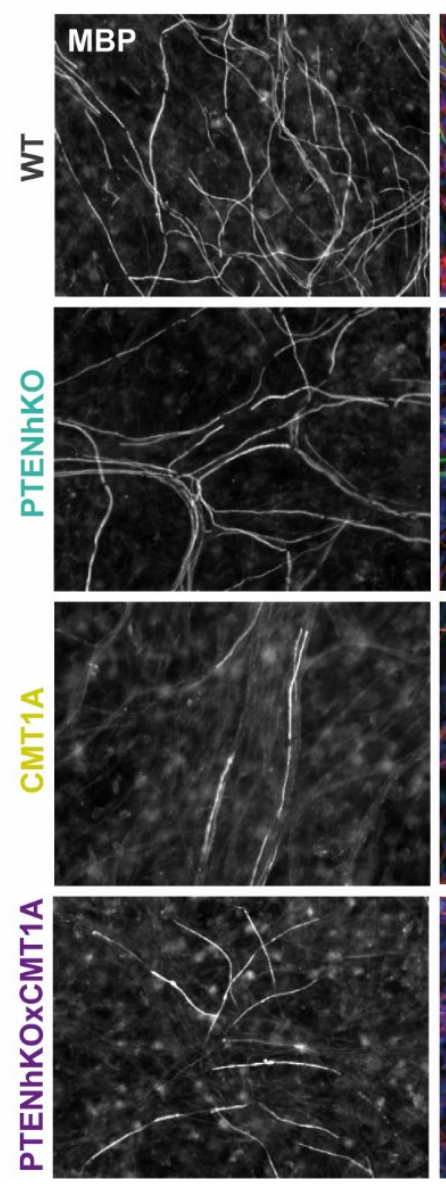
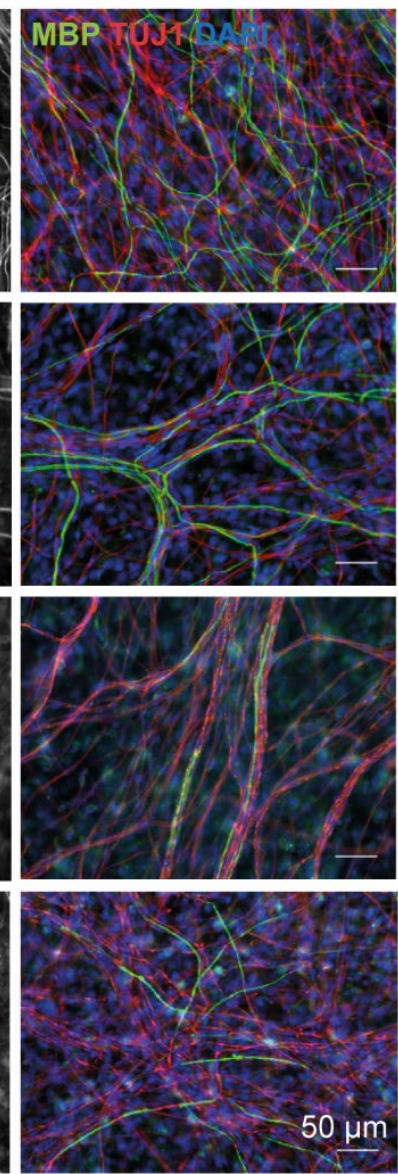

b

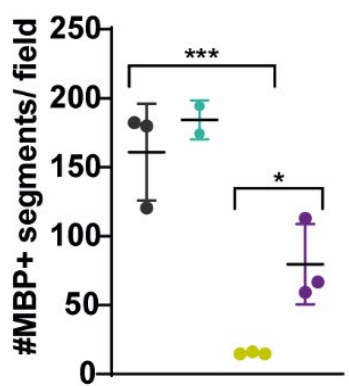

- WT

- PTENhKO

- CMT1A

- PTENhKOXCMT1A

C

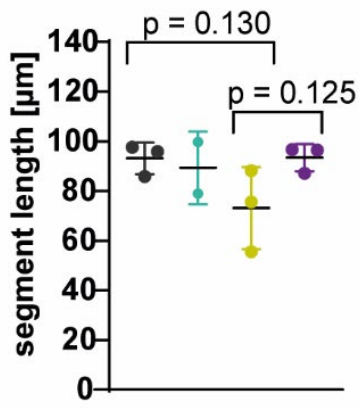

Figure 11: Increased myelination in PTENhKOxCMT1A double mutants in vitro.

(a) Example images of WT, PTENhKO, CMT1A and PTENhKOxCMT1A SC-DRG co-cultures (from top to bottom). Left panels show MBP positive myelinated segments (white), right panels show an overlay of MBP (myelin, green), TUJ1 (neuros, red) and DAPI (nuclei, blue). Scale bar is $50 \mu \mathrm{m}$.

(b) Quantification of myelinated segments per field display a strong decrease in cultures from CMT1A mice (yellow) compared to WT (grey) and PTENhKO (turquoise). Double mutants reveal an increase in the number of myelinated segments.

(c) Segment length tends to be decreased in CMT1A cultures, which is slightly increased in the double mutants.

Each data point represents one embryo. MBP positive segments and segment length were quantified in 5 fields (500 $\mathrm{x} 500 \mu \mathrm{m})$. Means are displayed \pm SD. Statistical analysis: One-way ANOVA with Sidak's multiple comparison test. ${ }^{*} \mathrm{p} \leq 0.05,{ }^{* *} \mathrm{p} \leq 0.01,{ }^{* * *} \mathrm{p} \leq 0.001$ and ${ }^{* * * *} \mathrm{p} \leq 0.0001$.

\section{Increased myelination in PTENhxCMT1A double mutants early in development.}

In vitro, we observed a positive effect of PTEN reduction on myelination in CMT1A. In a next step, we analyzed the impact of PTEN reduction in CMT1A in vivo.

Dividing the axon diameter by the fiber diameter including the myelin sheath results in the gratio, which is commonly used to describe the myelin sheath thickness. A g-ratio of 1 indicates the axon is not surrounded by myelin - it is amyelinated. The smaller the g-ratio, the thicker 
is the myelin. We analyzed g-ratios of axons in the motor femoral nerves of 18 days old mice, to evaluate effects of PTEN reduction in early development.

Already at postnatal day 18, CMT1A mice displayed an increase in amyelinated axons and smaller axon diameters compared to their respective WT controls (Figure 12b, d). The mean g-ratio is not significantly changed in CMT1A mice at this developmental stage (Figure 12c), however, plotting g-ratio against axon diameter revealed a clear shift in small caliber axons being hypermyelinated and big caliber axons being hypomyelinated with respect to their WT controls (Figure 12e). Heterozygous deletion of PTEN in Schwann cells (PTENhKO) alone, did not significantly alter the number of amyelinated axons, mean g-ratio or axon diameter compared to WT controls at postnatal day 18 (Figure 12a-d). Decreasing PTEN in CMT1A Schwann cells (PTENhKOxCMT1A) significantly reduced the amount of amyelinated axons (Figure 12b). Moreover, axon diameters did not change compared to CMT1A mice whereas the mean g-ratio was smaller in double mutants, indicating thicker myelin sheaths (Figure 12c-
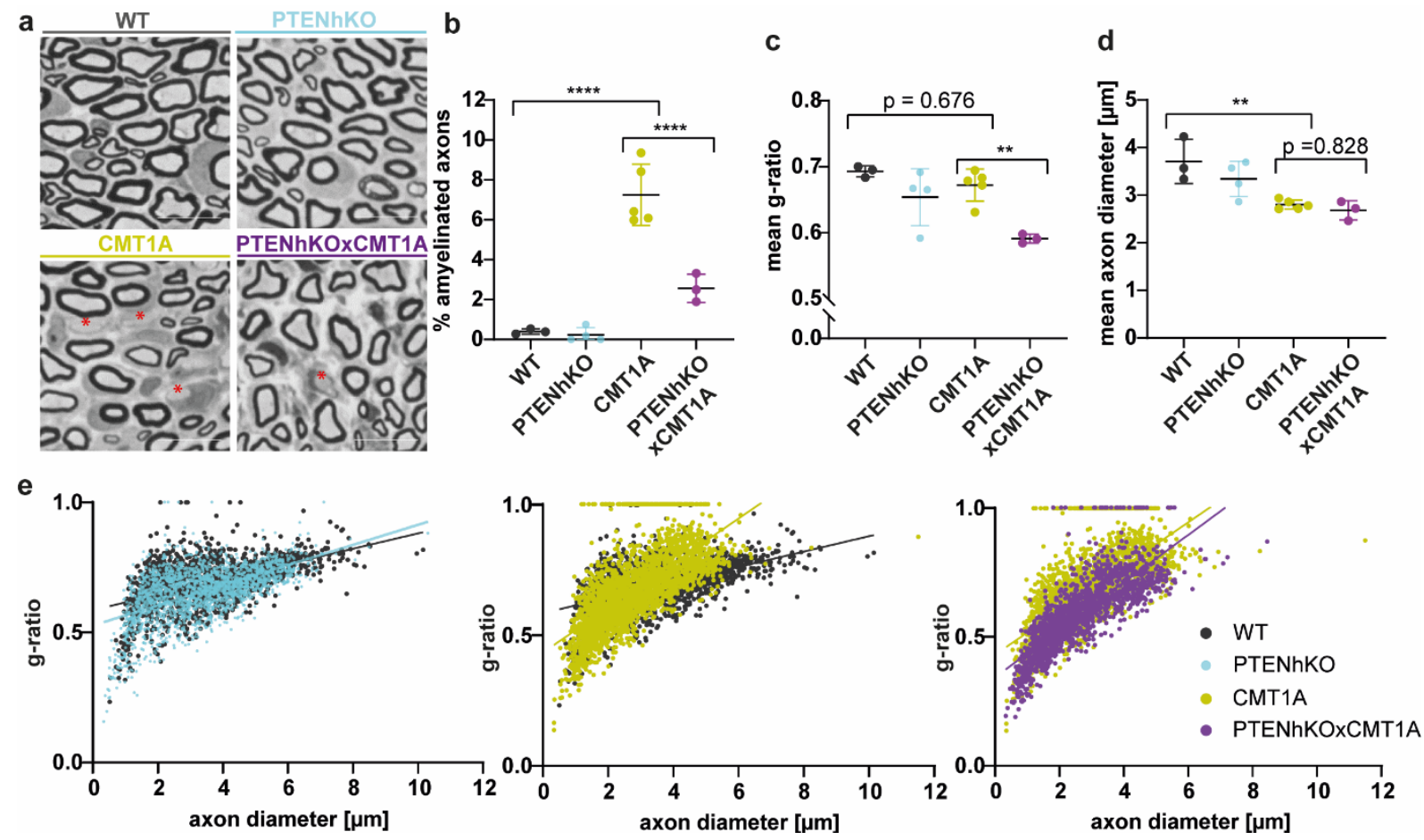

Figure 12: Increased myelination in PTENhxCMT1A double mutants at postnatal day 18.

(a) Example images of semi thin cross sections of WT (upper left), PTENhKO (upper right), CMT1A (lower left) and PTENhKOxCMT1A (lower right) femoral nerves. Red asterisks indicate amyelinated axons. Scale bar is 10 $\mu \mathrm{m}$.

(b) The percentage of amyelinated axons is decreased in PTENhKOxCMT1A double mutants (purple) compared to CMT1A mice (yellow), while PTENhKO (turquoise) and WT mice (grey) show no difference.

(c) PTENhKOxCMT1A double mutants display a smaller g-ratio compared to CMT1A mice.

(d) The mean axon diameter in PTENhKOxCMT1A double mutants is unchanged to CMT1A mice, which have a smaller axon diameter than the WT.

(e) From left to right: Scatter plots of WT (grey) and PTENhKO (turquoise), WT (grey) and CMT1A (yellow) as well as CMT1A (yellow) and PTENhKOxCMT1A (purple) axon diameters versus g-ratio. Solid lines display linear regressions.

In (b)-(d) each data point represents one animal. Whole femoral nerves were quantified. Means are displayed \pm SD. Statistical analysis: One-way ANOVA with Sidak's multiple comparison test. ${ }^{*} \mathrm{p} \leq 0.05,{ }^{* *} \mathrm{p} \leq 0.01,{ }^{* * *} \mathrm{p} \leq 0.001$ and ${ }^{* * * *} \mathrm{p} \leq 0.0001$. In (e) all quantified axons of $\mathrm{n}=3 \mathrm{WT}, \mathrm{n}=4$ PTENhKO, $\mathrm{n}=5$ CMT1A and $\mathrm{n}=3$ PTENhKOxCMT1A animals are displayed. 
e). Hence, activating the PI3K/Akt/mTOR pathway by reducing its main inhibitor PTEN in vivo in CMT1A, increased myelin sheath thickness early in development and partially prevented axons from being amyelinated.

Further, we wanted to elucidate whether the PTEN reduction in CMT1A Schwann cells does also influence myelination longitudinally. Therefore, we analyzed teased fiber preparations of sciatic nerves at postnatal day 18 for the internodal length and fiber diameter (Figure 13a). CMT1A mice displayed shortened internodes compared to WT controls, while PTENhKO mice were unaffected (Figure 13b). Mean fiber diameters were slightly smaller in CMT1A mice and unaltered in PTENhKOxCMT1A double mutants (Figure 13c). However, plotting internodal length versus fiber diameter displayed a slight shift towards longer internodes in double mutants (Figure 13d, right panel).

a

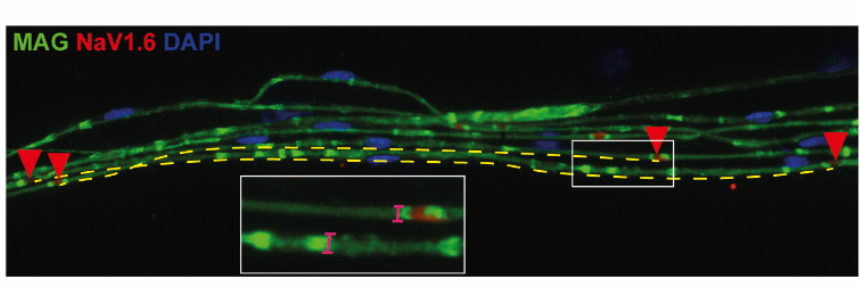

b

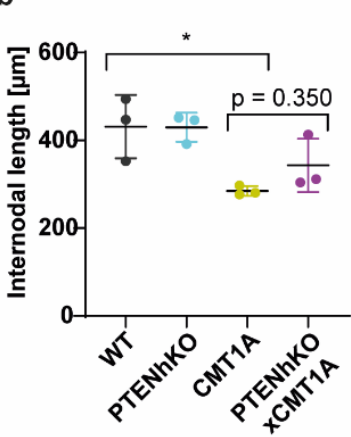

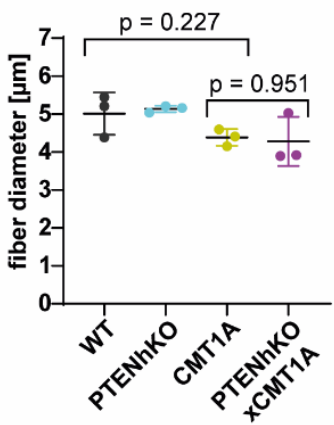
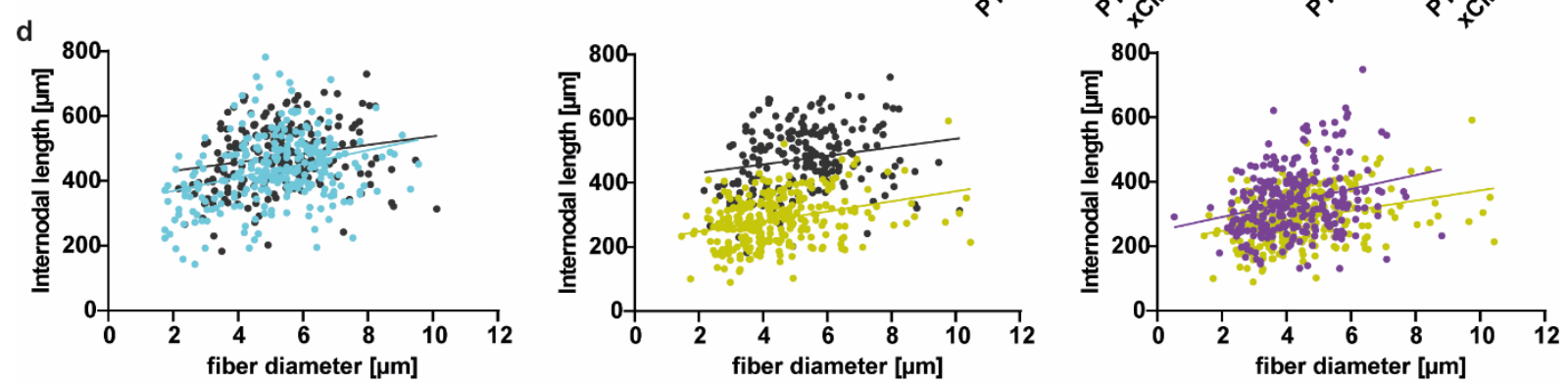

Figure 13: Internodes are slightly longer in PTENhKOxCMT1A double mutants at postnatal day 18.

(a) Example image of teased fiber preparations to analyze internodal length (yellow dashed line) and fiber diameter (magenta, blow-up image) by MAG (green) and NaV1.6 (red) staining. Nuclei are indicated by DAPI (blue).

(b) CMT1A mice (yellow) display shortened internodal length compared to WT controls (grey), while PTENhKO (turquoise) do not differ from WT. In PTENhKOxCMT1A mice (purple), internodes appear slightly longer than in CMT1A.

(c) Teased fiber diameters of CMT1A mice are slightly smaller than WT mice, although double mutants do not differ from CMT1A.

(d) From left to right: Scatter plot diagrams of WT (grey) and PTENhKO (turquoise), WT (grey) and CMT1A (yellow) as well as CMT1A (yellow) and PTENhKOxCMT1A (purple) internodal length against fiber diameter.

In (b)-(d) each data point represents one animal. 100 internodes were quantified per animal. Means are displayed \pm SD. Statistical analysis: One-way ANOVA with Sidak's multiple comparison test. ${ }^{*} \mathrm{p} \leq 0.05,{ }^{* *} \mathrm{p} \leq 0.01,{ }^{* * *} \mathrm{p} \leq 0.001$ and ${ }^{* * * *} \mathrm{p} \leq 0.0001$. In (e) all quantified internodes of $\mathrm{n}=3 \mathrm{WT}, \mathrm{n}=3$ PTENhKO, $\mathrm{n}=4$ CMT1A and $\mathrm{n}=3$ PTENhKOxCMT1A animals are displayed. Data was acquired with the help of Timon Hartmann (Master student)..

Therefore, PTEN reduction in CMT1A Schwann cells increased radial myelination and displayed trends towards improved longitudinal myelination early in development. The increased myelination with PTEN reduction in young animals prompted us to study later developmental stages as well as the effects of a full PTEN depletion in CMT1A. 


\section{Full PTEN knockout in CMT1A deteriorates the histological phenotype.}

PTEN conditional knockout mice show a highly increased activation of the PI3K/AKT/mTOR signaling pathway, which leads to the formation of recurrent myelin loops and tomacula (Goebbels et al 2012). We asked the question whether the heterozygous depletion is sufficient enough to counteract the downregulated PI3K/AKT/mTOR signaling pathway in CMT1A. Therefore, we generated a full PTEN knockout in CMT1A Schwann cells (Figure 14a). Semithin cross-sections showed increased numbers of amyelinated axons and aberrant myelin profiles in PTENfKOxCMT1A mice (Figure 14b). Moreover, teased fiber staining displayed tomacula formation at the paranodal area similar to PTENfKO mice (Figure 14c).

Taken together, a full PTEN knockout in CMT1A Schwann cells deteriorated the histological phenotype. Thus, we only analyzed PTEN heterozygous depletion in CMT1A Schwann cells in aged animals.

a

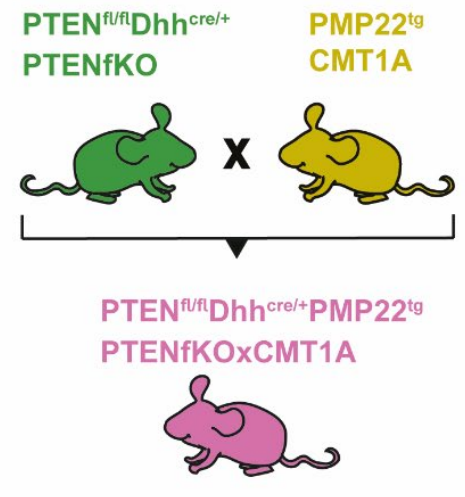

b

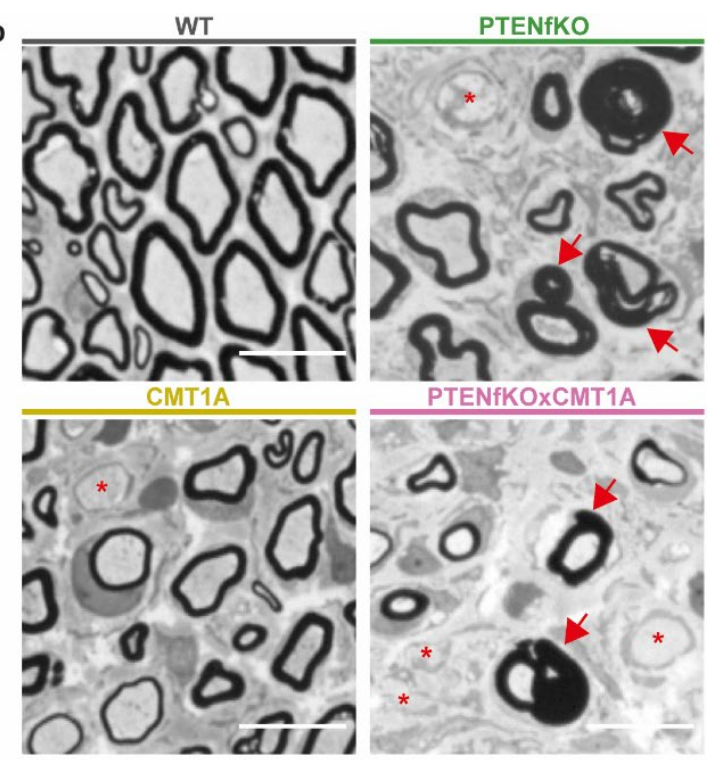

c
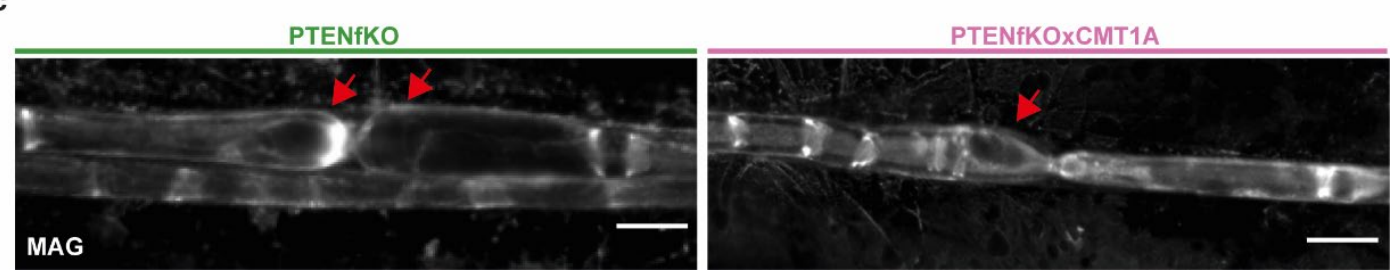

Figure 14: Full PTEN knockout in CMT1A Schwann cells deteriorates the histological phenotype.

(a) Crossing scheme of PTEN SC specific full knockout mice (PTENfKO, green) with CMT1A mice (CMT1A, yellow) to generate double mutants (PTENfKOxCMT1A, pink).

(b) Example images of semi thin cross sections of WT (upper left), PTENfKO (upper right), CMT1A (lower left) and PTENfKOxCMT1A (lower right) femoral nerves. Red asterisks indicate amyelinated axons and arrows aberrant myelin profiles. Scale bar is $10 \mu \mathrm{m}$.

(c) Teased fibers of PTENfKO (left panel) and PTENfKOxCMT1A mice (right panel) show tomacula formation in the paranodal area as indicated by arrows. Scale bar is $20 \mu \mathrm{m}$. 


\subsubsection{PTEN reduction in CMT1A does not alter the phenotype in aged animals.}

The effects of PTEN reduction in CMT1A Schwann cells early in development were characterized by a reduction in amyelinated axons, thicker myelin sheaths and slightly increased internodal length, although PTENhKOxCMT1A mice never reached WT levels. We

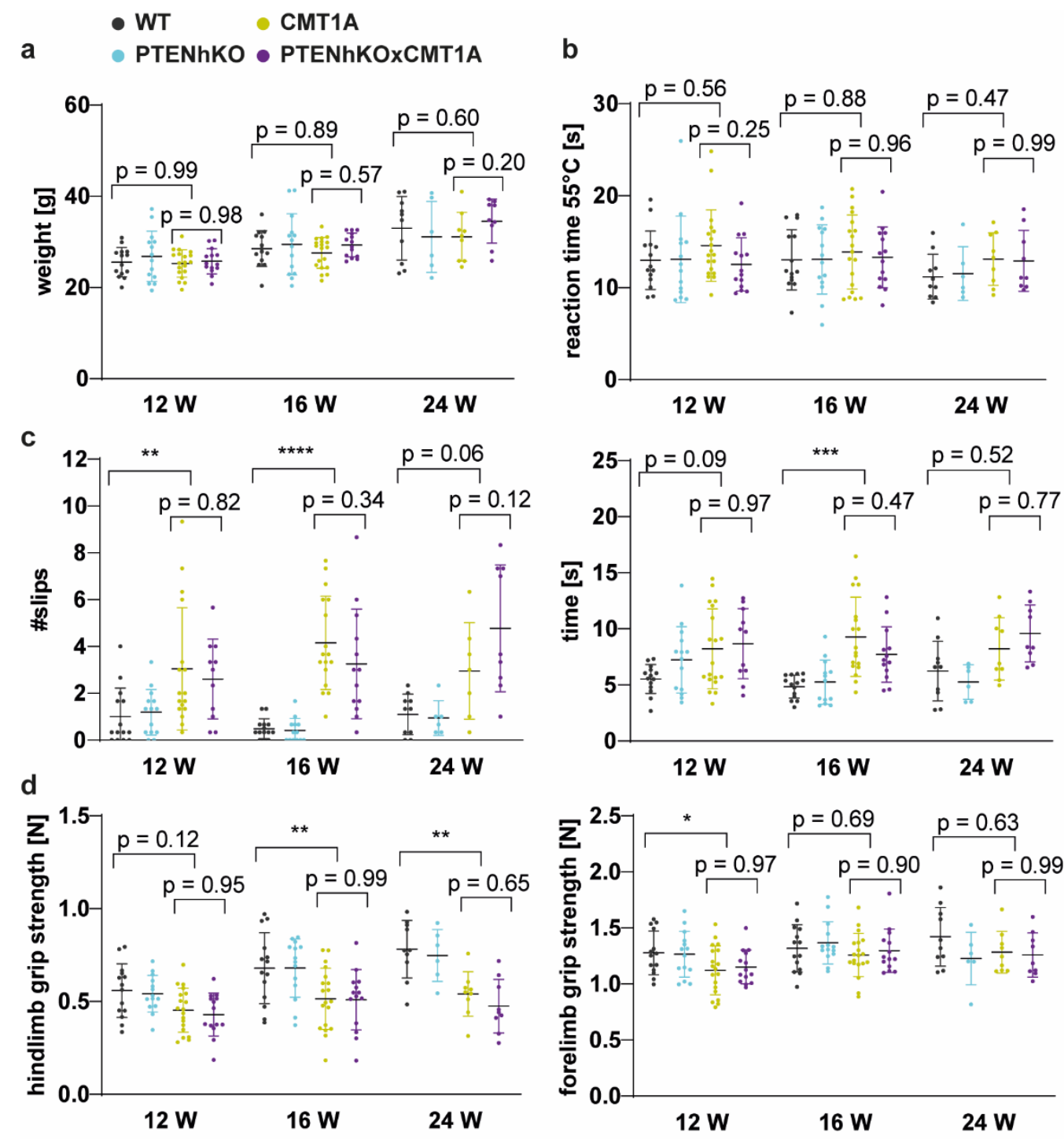

Figure 15: Motor and sensory behavior is not altered in PTENhKOxCMT1A double mutants.

(a) Weight of WT (grey), PTENhKO (turquoise), CMT1A (yellow) and PTENhKOxCMT1A (purple) mice at 12, 16 and 24 weeks. Weight gain over time is observed in all groups, while the genotypes do not differ.

(b) The reaction time on the hotplate is unchanged in all groups at all time points.

(c) CMT1A mice display a significant increase in the number of slips on the elevated beam compared to WT controls. PTENhKOxCMT1A double mutants trend to slip more often at 24 weeks of age (left panel). Moreover, CMT1A mice take longer running up the beam compared to WT controls, while PTENhKOxCMT1A mice are unchanged.

(d) Grip strength force is less in CMT1A mice hindlimbs, while PTENhKOxCMT1A did not change (left panel). Forelimb grip strength is slightly reduced in CMT1A mice in comparison to WT controls and unchanged in double mutants (right panel).

Means are displayed \pm SD. WT $n=10-14$, PTENhKO $n=6-14$, CMT1A $n=9-19$, PTENhKOxCMT1A $n=9-14$ mice were analyzed at 16 weeks of age. Statistical analysis: One-way ANOVA with Sidak's multiple comparison test. ${ }^{*} \mathrm{p} \leq 0.05,{ }^{* *} \mathrm{p} \leq 0.01,{ }^{* * *} \mathrm{p} \leq 0.001$ and ${ }^{* * * *} \mathrm{p} \leq 0.0001$. 
further assessed histological, phenotypical and electrophysiological components also in aged animals.

\section{Motor behavior and electrophysiological properties are not altereded in adult PTEN- depleted CMT1A mice.}

Phenotypical analysis revealed that the animals gained normal weight from 12 to 24 weeks and did not display difference between the genotypes (Figure 15a). Furthermore, we assessed the motor behavior phenotype by the elevated beam and grip strength test. CMT1A mice slipped more often on the elevated beam and needed longer to run $80 \mathrm{~cm}$ (Figure 15c). Moreover, CMT1A mice displayed a reduced force in their hind- and forelimbs compared to WT controls (Figure 15d). Although the PTENhKOxCMT1A double mutants showed a

a

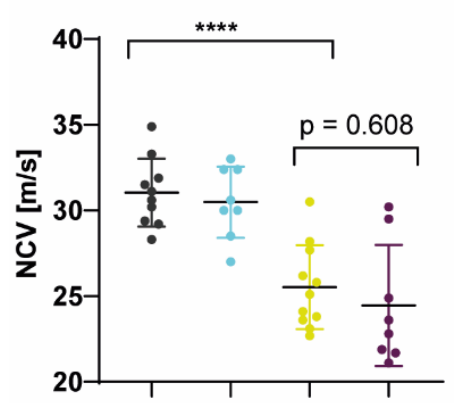

d

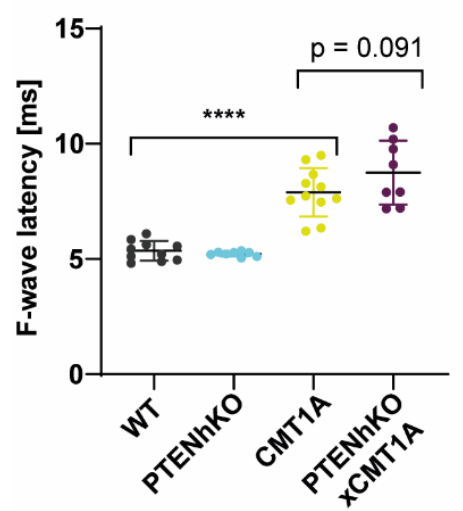

b

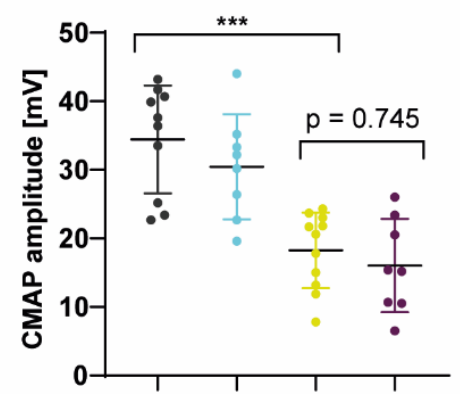

e

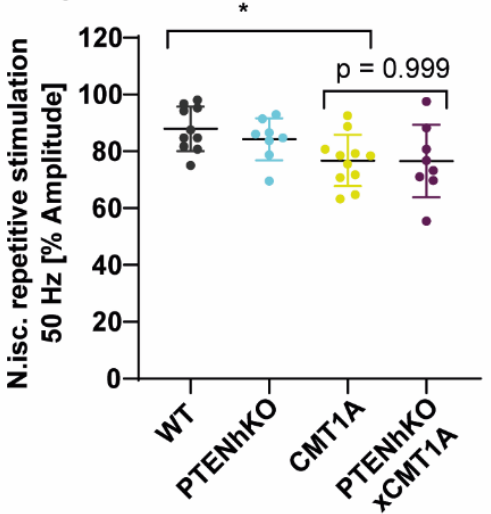

c

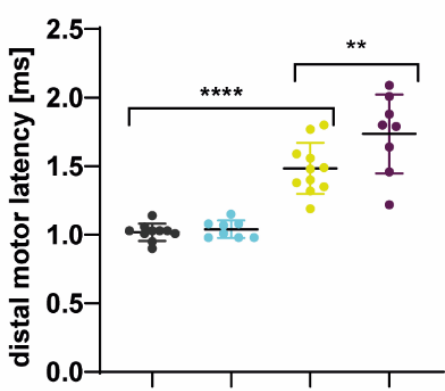

f

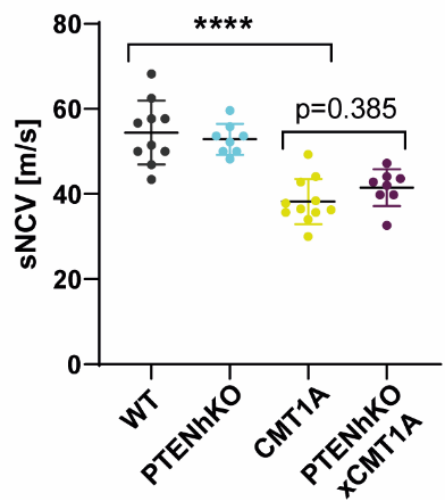

Figure 16: Electrophysiological parameters are unaltered in PTENhKOxCMT1A double mutants.

(a) CMT1A mice display slower nerve conduction velocity than WT controls at 16 weeks, whole double mutants are unchanged

(b) The analysis of compound muscle action potential amplitude displays no significant changes between WT and PTENhKO, while CMT1A mice amplitudes are significantly lower. When PTEN is reduced in CMT1A mice, amplitudes do not differ from CMT1A mice.

(c) CMT1A mice display a prolonged distal motor latency compared to WT controls (grey) which is significantly longer in CMT1A mice with reduced PTEN.

(d) F-wave latency is significantly longer in CMT1A mice compared to WT controls. Thereby, PTENhKOxCMT1A mice show a trend towards prolonged latency compared to CMT1A mice.

(e) Repetitive stimulation with a frequency of $50 \mathrm{~Hz}$ shows reduced amplitudes in CMT1A mice.

(f) CMT1A mice reveal a significantly slower sensory nerve conduction velocity than WT controls. Double mutants do not differ.

Means are displayed \pm SD. WT $n=10$, PTENhKO $n=8, C M T 1 A n=11$, PTENhKOxCMT1A $n=8$ mice were analyzed at 16 weeks of age. Statistical analysis: One-way ANOVA with Sidak's multiple comparison test. ${ }^{*} \mathrm{p} \leq 0.05,{ }^{* *} \mathrm{p} \leq$ $0.01,{ }^{* * *} \mathrm{p} \leq 0.001$ and ${ }^{* * *} \mathrm{p} \leq 0.0001$. Theresa Kungl performed electrophysiological measurements. 
tendency towards reduced number of slips at 12 and 16 weeks, at 24 weeks this trend was not visible anymore (Figure 15c,d). Also the hind limb grip strength of double mutants was unaltered as compared with CMT1A mice, and at 24 weeks there was even a tendency towards reduced grip strength in the double mutants (Figure 15d). Furthermore, we made use of the Hotplate test to analyze sensory response to heat stimuli in mice. We did not observe significant alterations in reaction time between the genotypes, although CMT1A mice showed tendencies to react slower on the hotplate (Figure 15b).

We also did electrophysiological studies at 16 weeks of age. The measurements revealed a slower nerve conduction velocity, decreased amplitude and prolonged latencies in CMT1A mice (Figure 16a-c). Similar to the behavioral phenotype, PTENhKOxCMT1A double mutants did not improve electrophysiologically and distal motor latency was even prolonged in the

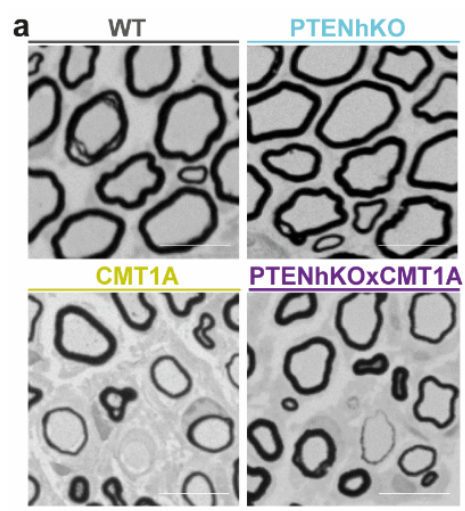

b
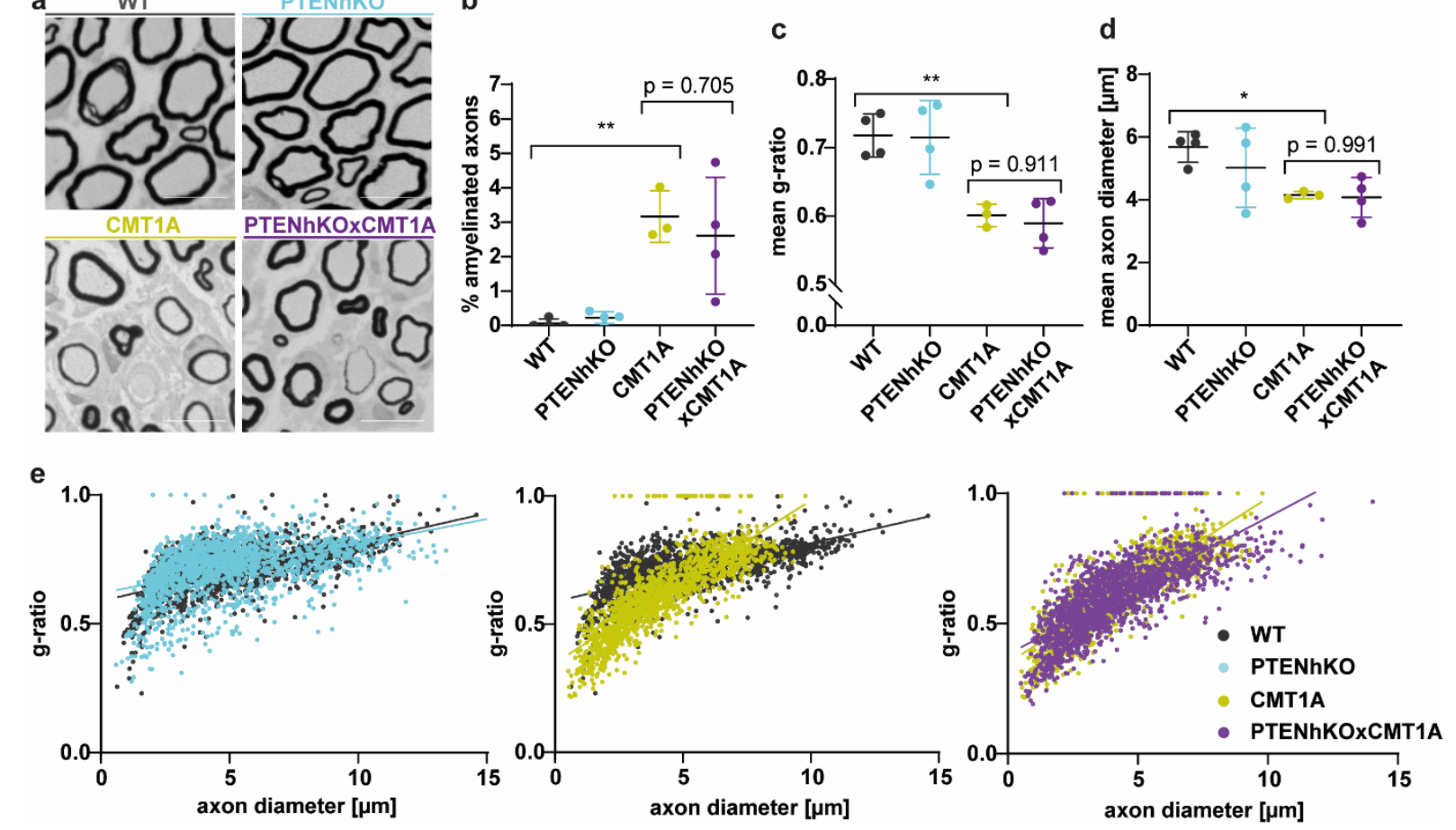

Figure 17: Histologically, PTENhKOxCMT1A double mutants do not differ from CMT1A mice at 16 weeks of age.

(a) Example images of semi thin cross sections of WT (upper left), PTENhKO (upper right), CMT1A (lower left) and PTENhKOxCMT1A (lower right) femoral nerves. Scale bar is $10 \mu \mathrm{m}$.

(b) CMT1A mice (yellow) display an increase in amyelinated axons compared to WT (grey) mice, while PTENhKOxCMT1A double mutants (purple) are indifferent from CMT1A mice at 16 weeks of age.

(c) PTENhKOxCMT1A double mutants display unchanged g-ratio compared to CMT1A mice, which show a decreased g-ratio compared to WT controls.

(d) The mean axon diameter in PTENhKOxCMT1A double mutants is unchanged to CMT1A mice, which have a smaller axon diameter than the WT.

(e) From left to right: Scatter plots of WT (grey) and PTENhKO (turquoise), WT (grey) and CMT1A (yellow) as well as CMT1A (yellow) and PTENhKOxCMT1A (purple) axon diameters versus g-ratio. Solid lines display linear regressions.

In (b)-(d) each data point represents one animal. Whole femoral nerves were quantified. Means are displayed \pm SD. Statistical analysis: One-way ANOVA with Sidak's multiple comparison test. ${ }^{*} \mathrm{p} \leq 0.05,{ }^{* *} \mathrm{p} \leq 0.01,{ }^{* * *} \mathrm{p} \leq 0.001$ and ${ }^{* * * *} \mathrm{p} \leq 0.0001$. In (e) all quantified axons of $\mathrm{n}=4 \mathrm{WT}, \mathrm{n}=4$ PTENhKO, $\mathrm{n}=3$ CMT1A and $\mathrm{n}=4$ PTENhKOxCMT1A animals are displayed. 
animals (Figure 16c). Not only motor, but also sensory nerve conduction velocity was reduced in CMT1A mice and did not changed in PTEN reduced CMT1A mice (Figure 16f).

To sum up, PTEN reduction in CMT1A Schwann cells neither resulted in behavioral nor electrophysiological improvements in adult mice, with some parameters even showing a deteriorated phenotype in older PTENhKOxCMT1A double mutants.

PTENhKOxCMT1A mice display unaltered myelin sheath thickness compared to CMT1A mice.

The previously observed reduction in amyelinated axons and shift towards thicker myelin sheaths at postnatal day 18 was not visible at 16 weeks of age (Figure 17). In the histological analysis, double mutants did not differ from CMT1A mice in the percentage of amyelinated axons, mean g-ratio and axon diameter (Figure 17a-d). In addition, the relative distribution of PTENhKOxCMT1A axons to g-ratio is unaltered at 16 weeks (Figure 17f). The early effect of increased myelin sheath thickness is not visible in 16 weeks old animals.

a
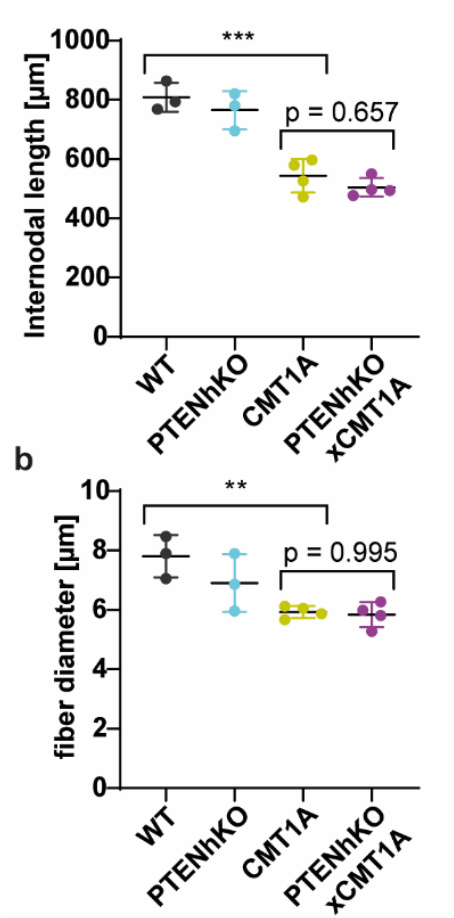

c
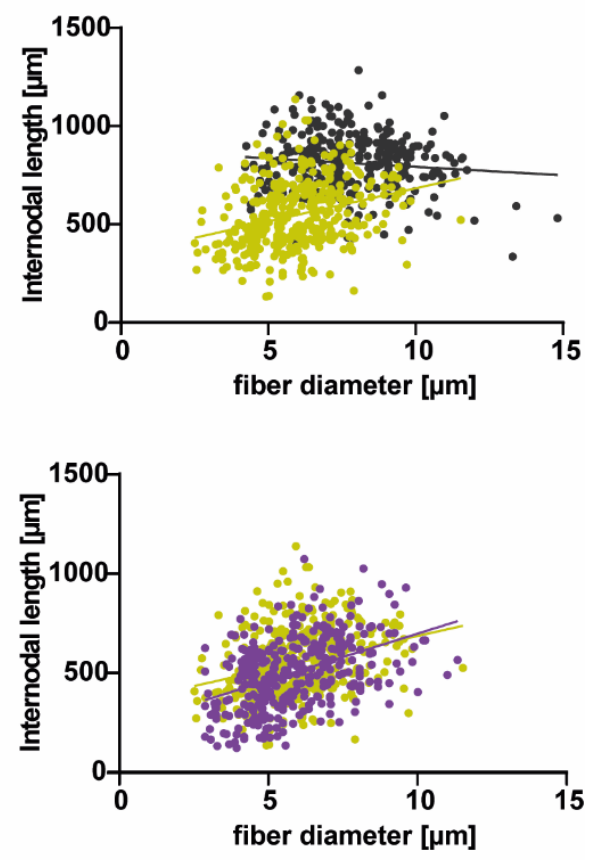

Figure 18: Longitudinal myelination is unaltered in PTENhKOxCMT1A double mutants at 16 weeks.

(a) CMT1A mice (yellow) display shortened internodal length compared to WT controls (grey), while PTENhKO (turquoise) do not differ from WT. In PTENhKOxCMT1A mice (purple), internodes internodal length is unaltered compared to CMT1A mice.

(b) Teased fiber diameters of CMT1A mice are significantly smaller than in WT mice, although double mutants do not differ from CMT1A.

(c) Scatter plot diagrams of WT (grey) and CMT1A (yellow) as well as CMT1A (yellow) and PTENhKOxCMT1A (purple) internodal length against fiber diameter.

In (b)-(d) each data point represents one animal. 100 internodes were quantified per animal. Means are displayed \pm SD. Statistical analysis: One-way ANOVA with Sidak's multiple comparison test. ${ }^{*} p \leq 0.05,{ }^{* *} p \leq 0.01,{ }^{* * *} p \leq 0.001$ and ${ }^{* * * *} \mathrm{p} \leq 0.0001$. In (e) all quantified internodes of $\mathrm{n}=3 \mathrm{WT}, \mathrm{n}=3$ PTENhKO, $\mathrm{n}=4$ CMT1A and $\mathrm{n}=4$ PTENhKOxCMT1A animals are displayed. . Data was acquired with the help of Timon Hartmann (Master student). 
Moreover, at postnatal day 18, PTENhKOxCMT1A mice displayed a trend towards longer internodes than CMT1A mice. Therefore, we analyzed internodal length in 16 weeks old teased fiber preparations and could not observe difference in internodal length and fiber diameter between CMT1A und PTEN reduced CMT1A mice (Figure 18).

Furthermore, we investigated the histological effects of PTEN reduction in 24 weeks old animals. CMT1A mice displayed an increase in amyelinated axons, which tended to be even more pronounced in PTENhKOxCMT1A mice (Figure 19a,b). Moreover, mean g-ratios and axon diameters were not altered compared to CMT1A mice (Figure 19c-e).
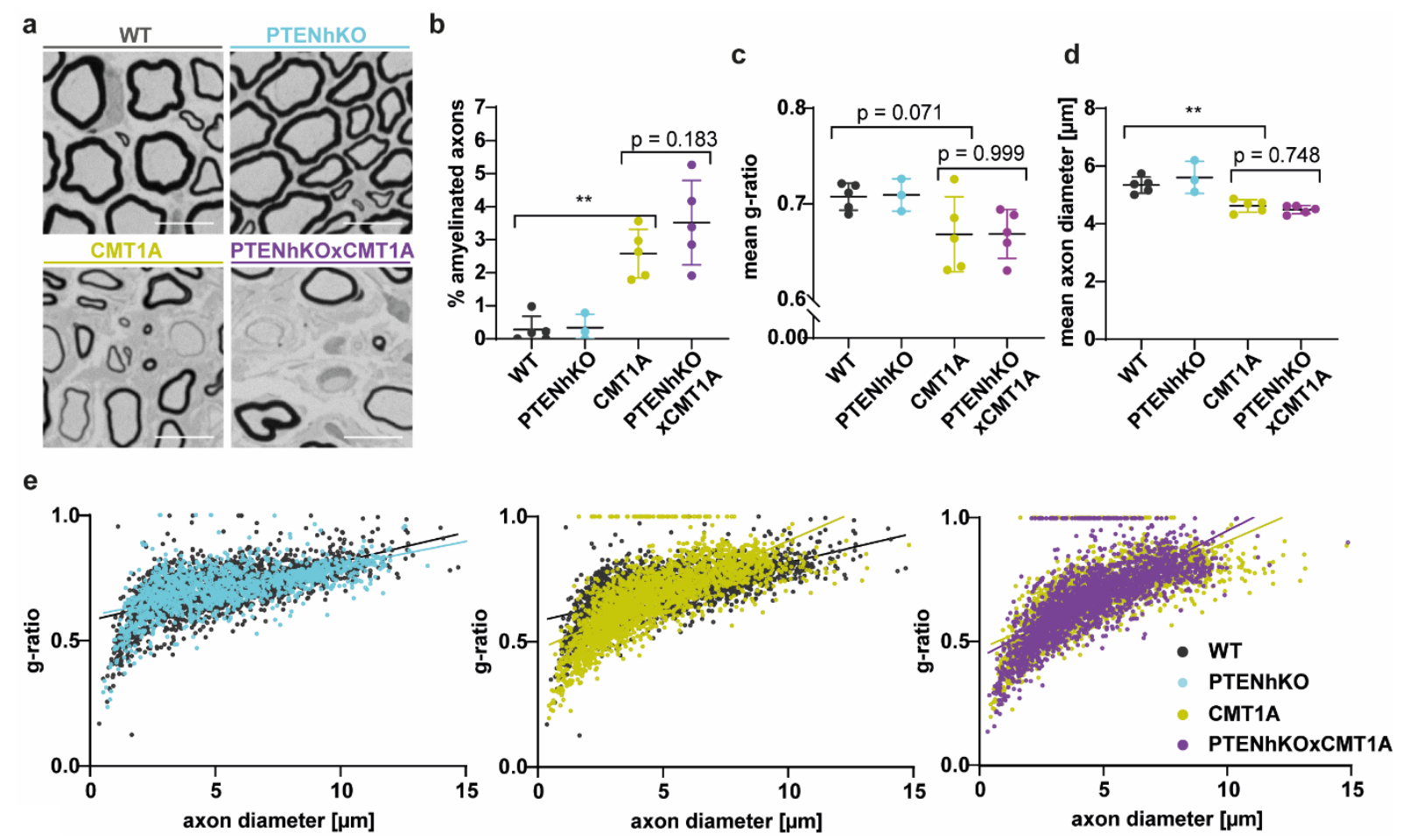

Figure 19: PTENhKOxCMT1A double mutants are unaltered at 24 weeks of age.

(a) Example images of toluidine blue and gallays stained semi thin cross sections of WT (upper left), PTENhKO (upper right), CMT1A (lower left) and PTENhKOxCMT1A (lower right) femoral nerves. Scale bar is $10 \mu \mathrm{m}$.

(b) CMT1A mice (yellow) display an increase in amyelinated axons compared to WT (grey) mice, while PTENhKOxCMT1A double mutants (purple) show slightly more amyelinated axons than CMT1A mice at 24 weeks of age.

(c) PTENhKOxCMT1A double mutants display unchanged g-ratio compared to CMT1A mice, which show a decreased g-ratio compared to WT controls.

(d) The mean axon diameter in PTENhKOxCMT1A double mutants is unchanged to CMT1A mice, which have a smaller axon diameter than the WT controls.

(e) From left to right: Scatter plots of WT (grey) and PTENhKO (turquoise), WT (grey) and CMT1A (yellow) as well as CMT1A (yellow) and PTENhKOxCMT1A (purple) axon diameters versus g-ratio. Solid lines display linear regressions.

In (b)-(d) each data point represents one animal. Whole femoral nerves were quantified. Means are displayed \pm SD. Statistical analysis: One-way ANOVA with Sidak's multiple comparison test. ${ }^{*} p \leq 0.05,{ }^{* *} p \leq 0.01,{ }^{* * *} p \leq 0.001$ and **** $\mathrm{p} \leq 0.0001$. In (e) all quantified axons of $\mathrm{n}=5 \mathrm{WT}, \mathrm{n}=3$ PTENhKO, $\mathrm{n}=5$ CMT1A and $\mathrm{n}=5$ PTENhKOxCMT1A animals are displayed.

Thus, counteracting the downregulated PI3K/Akt/mTOR pathway by genetic PTEN reduction in CMT1A increased myelin sheath thickness and decreased the amount of amyelinated axons early in development at postnatal day 18. In adult mice at 16 weeks of age, this was not visible 
anymore. In more aged animals at 24 weeks, the number of amyelinated axons even tended to be increased. These results are supported by phenotypical and electrophysiological analysis of the adult animals.

In the beginning we raised the questions whether (i) targeting PTEN and the downstream signaling pathway pharmacologically can be used as a therapeutic target and (ii) how PMP22 influences PTEN amounts in the Schwann cell. Indeed, using the PI3K/Akt/mTOR pathway as a therapeutical target was helpful in HNPP mice in vitro and in vivo, while modulation of the pathway in CMT1A by reducing PTEN amounts pharmacologically and genetically was beneficial for myelination in CMT1A in vitro and early at postnatal day 18 in vivo, but deteriorated the phenotype in older animals. Thus, the in vivo situation seems to be more complex. The results suggest a threshold amount of PTEN above the remaining amounts in PTEN-reduced Schwann cells that is needed for keeping up peripheral nerve function in later development of diseased animals. Under healthy conditions however, this threshold amount of PTEN seems to be lower, because the phenotype of PTENhKO mice was unaltered in comparison with the wildtype. 


\subsection{Vinculin as a linker between PMP22 and growth signaling?}

Modulating the PTEN/PI3K/Akt/mTOR signaling pathway in animal models of HNPP and CMT1A opens up new therapeutical targets in PMP22 gene-dosage dependent diseases. An unsolved question is the connection between PMP22 and growth signaling pathway. Does PMP22 interact with signaling molecules such as PTEN, either directly or indirectly?

We tried to shed light on these questions by performing crosslinking and immunoprecipitation experiments, which did not reveal a direct interaction of PMP22 and growth signaling molecules. Nevertheless, these experiments suggested focal adhesion proteins as potential interaction partners of PMP22 (David Ewers, Friederike Arlt, unpublished data). One interesting candidate among these is the actin-binding protein Vinculin, which localizes at focal adhesions and cadherin-mediated cell-cell junctions. Vinculin was reported to protect PTEN from degradation on protein level [Subauste et al 2005], opening up the possibility that interaction of PMP22 with focal adhesion components leads to higher PTEN levels upon PMP22 expression. In line with this hypothesis, on protein level CMT1A nerves displayed more and HNPP less Vinculin, reminiscent of our previous finding of altered PTEN protein levels (Figure 20). These results prompted us to further study Vinculin function in the Schwann cell and possible implications in CMT1A.

a

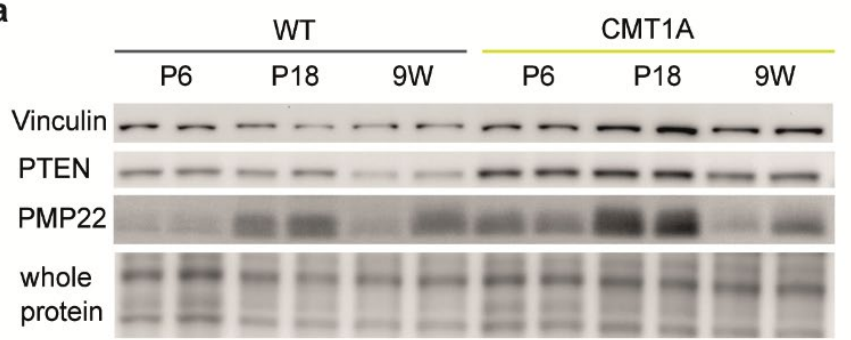

b

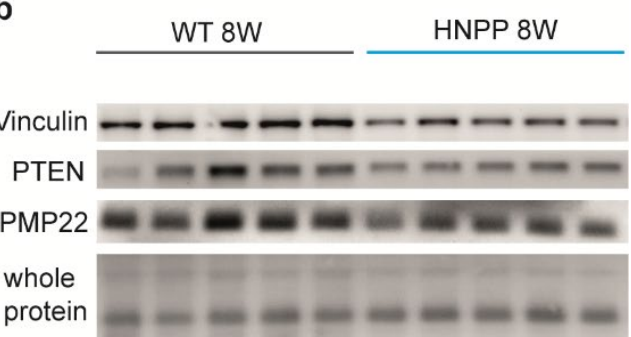

Figure 20: Vinculin is PMP22 dosage-dependently changed in animal models of CMT1A and HNPP.

(a) Immunoblot analysis of whole sciatic nerve lysates from postnatal day 6, 18 and 9 weeks old WT (grey) and CMT1A (yellow) nerves showing increased Vinculin protein levels in CMT1A. Fast green whole protein staining as loading control.

(b) Immunoblot analysis of whole sciatic nerve lysates from 8 weeks old WT (grey) and HNPP (blue) nerves showing decreased Vinculin protein levels in HNPP. Fast green whole protein staining as loading control.

\subsubsection{Vinculin localizes to focal adhesions in primary Schwanns cell in vitro and paranodal loops as well as Cajal bands in vivo.}

We know only little about Vinculin's function in the Schwann cell. Vinculin has been detected in peripheral myelin (Siems et al 2020) and autoantibodies against Vinculin were enriched in patients suffering from a chronic inflammatory demyelinating polyneuropathy (Beppu et al 2015), hinting towards an important, yet unknown, role in the Schwann cell.

First, we aimed at analyzing Vinculin expression and localization in the wildtype Schwann cell and then further investigated changes in CMT1A.

In rat primary Schwann cell cultures, Vinculin strongly localized to focal adhesion plaques at the polarized, actin-rich lamellipodia (Figure 21a). In the more complex in vivo situation, 
a

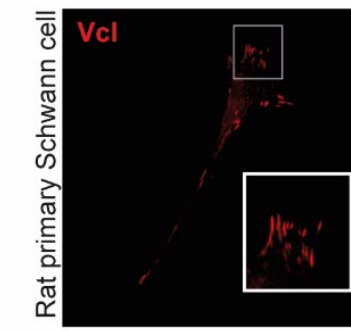

b

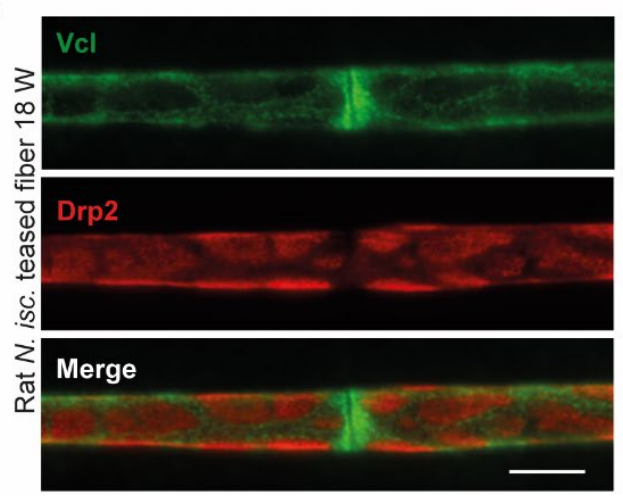

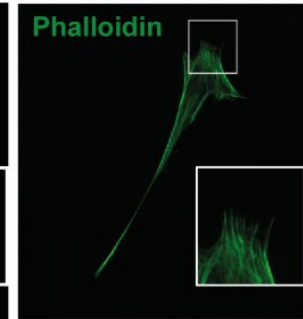

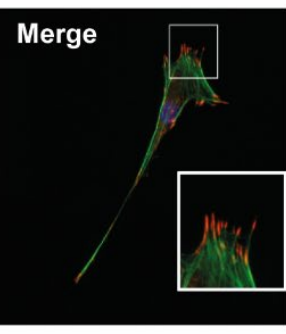

C

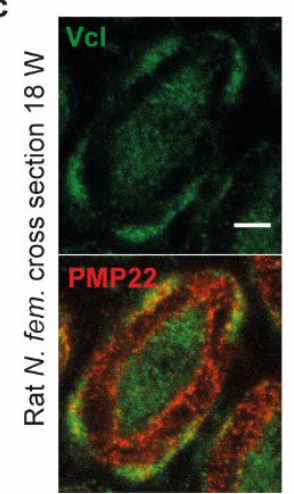

d

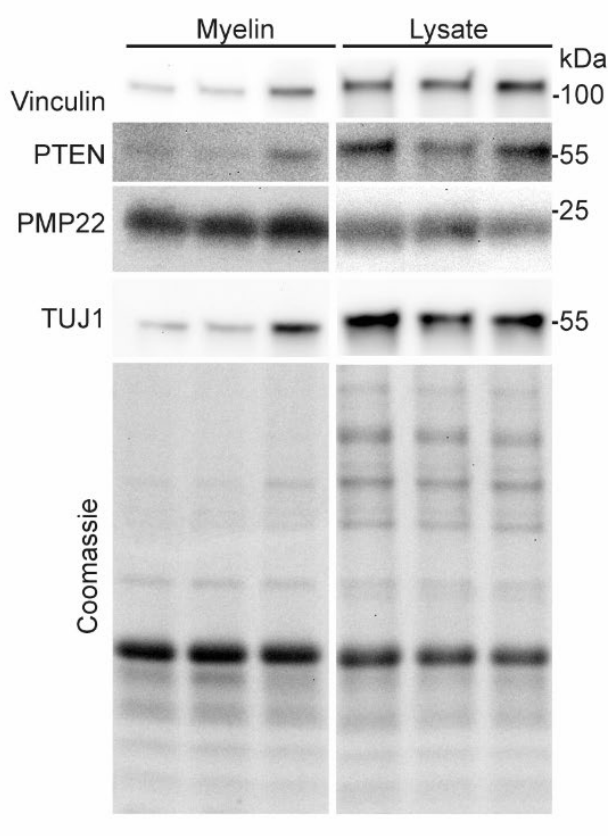

Figure 21: Vinculin localizes to focal adhesions in vitro and bands of Cajal in the Schwann cell in vivo.

(a) Vinculin (left panel, red) localizes to focal adhesion plaques (inset) of rat primary Schwann cells in vitro (2 days, + FSK +BPE), costained with Actin marker Phalloidin (middle panel, green).

(b) In teased fiber preparations of 18 weeks old wildtype rat sciatic nerves Vinculin expression (upper panel, green) is restricted to the bands of Cajal and paranodal area of the Schwann cell. Drp2 is a marker for Appositions (middle panel, red). Scale bar is $10 \mu \mathrm{m}$.

(c) In STED imaged cross sections of 18 weeks old rat femoral nerves Vinculin (green, upper panel) is predominantly visible in the bands of Cajal and the axon. PMP22 is a marker for compact myelin (red, lower panel). Scale bar is $2 \mu \mathrm{m}$. STED imaging was performed by Joris van Dort.

(d) Immunoblot of wildtype p18 rat whole sciatic nerve lysate and myelin. Vinculin, PTEN and TUJ1 are enriched in Schwann cell lysate compared to the myelin fraction, in which PMP22 is enhanced. Coomassie staining is used as the loading control. Myelin purification from sciatic nerves was performed by David Ewers.

Vinculin immunoreactivity appeared at the paranodal region and abaxonally at the bands of Cajal (Figure $\mathbf{2 1 b}, \mathbf{c}$ ). Vinculin is predominantly associated with these structures outside the compact myelin and therefore enriched in lysate compared to myelin preparations of peripheral nerves, similar to axonal protein TUJ1 and cytoplasmic signaling protein PTEN (Figure 21d). PMP22 is mainly expressed in the compact myelin sheath while Vinculin resides in the abaxonal non-compact compartments (Figure 21c). A direct colocalization of both proteins was not observed, but there might be the possibility of a protein-protein interaction of Vinculin in the abaxonal membrane and PMP22 in the outermost layer of the compact myelin sheath.

\subsubsection{CMT1A Schwann cells are less polarized and migrate slower.}

As a focal adhesion protein, Vinculin is important in directing cellular shape to provide proper migration of the cell. Vinculin overexpressing cells migrate slower and are less motile in vitro (Rodriguez Fernandez et al 1992). In agreement with a possible interaction of PMP22 with focal adhesions, overexpression of PTEN (Tamura et al 1998) as well as PMP22 (Brancolini et al 
a

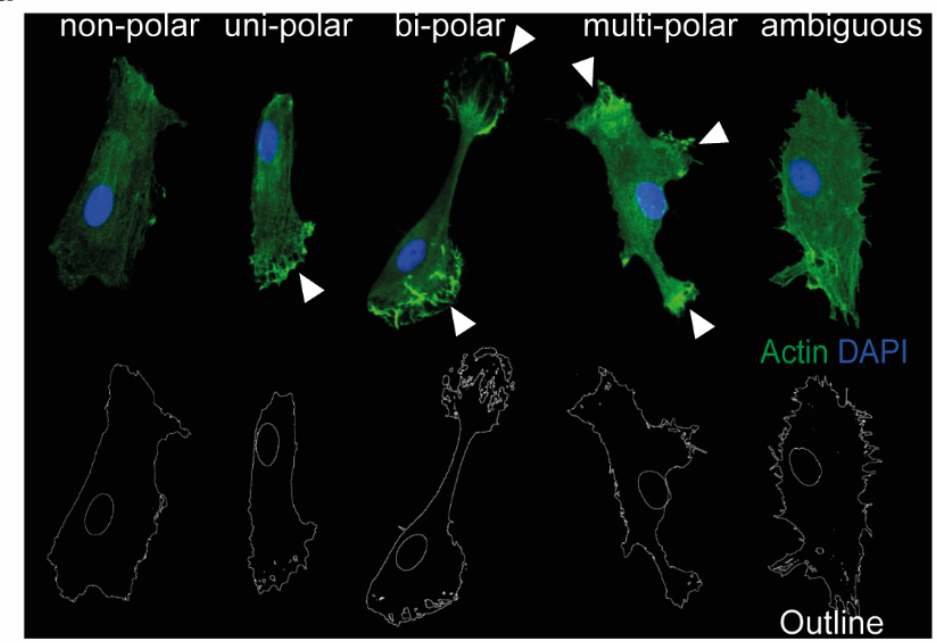

C

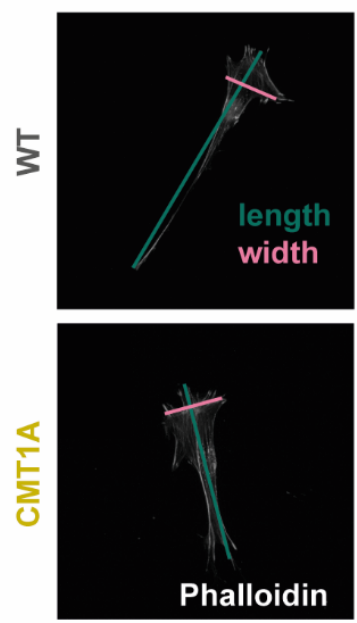

d

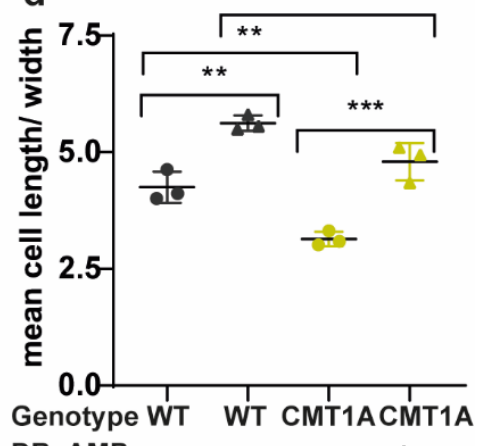

b

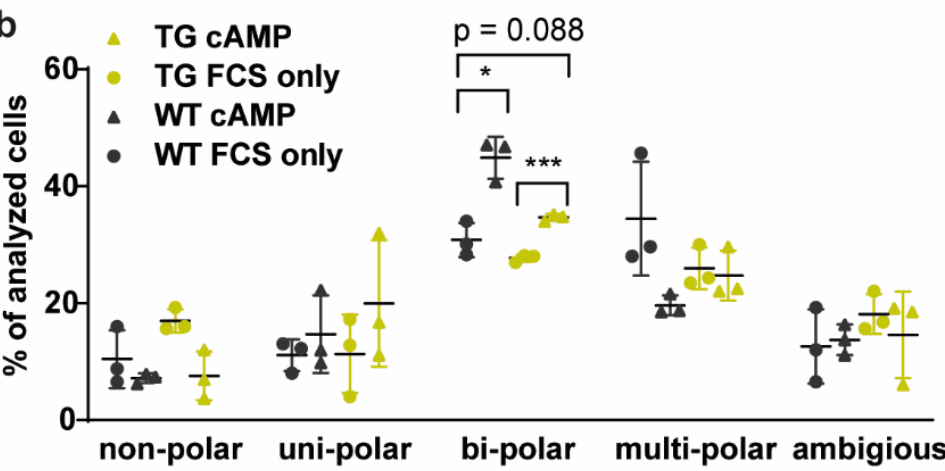

DBCAMP

Figure 22: CMT1A primary Schwann cells are less polarized.

(a) Example immunofluorescence images of rat wildtype primary Schwann cells in FCS displaying the analyzed polarity groups (from left to right): non-, uni-, bi-, multi-polar and ambiguous. White arrowheads indicate dense Actin (green) as a sign for polarized membranes.

(b) Most Schwann cell displayed a bi-polar shape, while stimulated cells display significantly more cells in the bipolar shape. Stimulated CMT1A Schwann cells are slightly less polarized than WT cells.

(c) Example immunofluorescence images of the length (green) to width (pink) measurements.

(d) Mean cell length to width ratio is increased after DBcAMP stimulation, while CMT1A primary Schwann cells are less polarized than wildtype controls.

Per genotype and condition $n=3$ biological replicates (primary Schwann cell preparations) were quantified with each 50 technical replicates (single Schwann cells). Means are displayed \pm SD. Statistical analysis: Two-way RM ANOVA with Tukey's multiple comparison test (b). One-way ANOVA with Sidak's multiple comparison test (d). ${ }^{*} \mathrm{p} \leq 0.05,{ }^{* *} \mathrm{p} \leq 0.01,{ }^{* * *} \mathrm{p} \leq 0.001$ and ${ }^{* * * *} \mathrm{p} \leq 0.0001$.

1999) have been shown to change cell shape and migration behavior in fibroblasts in vitro. Lee et al 2014 showed alterations in lampellipodia spreading and migration in PMP22 knockout Schwann cells. Therefore, we investigated the effects of PMP22 overexpression on cell shape and migration behavior in primary Schwann cells derived from CMT1A rats in vitro.

Rat primary Schwann cells from WT and CMT1A animals were kept under normal and DBcAMP stimulated conditions for $48 \mathrm{~h}$. After fixation, the cells were stained with Actin or Phalloidin to visualize the cytoskeleton and then grouped according to their shape in (i) nonpolar, (ii) uni-polar, (iii) bi-polar, (iv) multi-polar and (v) ambiguous morphology (Figure 22a). Most of the cells appeared bi-polar in the typical spindle-shaped Schwann cell form and the percentage of bi-polar cells significantly increased after stimulation (Figure 22b). Moreover, 
stimulated WT primary Schwann cells appeared stronger polarized that stimulated CMT1A Schwann cells (Figure 22b). To add a quantitative measure to the morphological analysis, we calculated the cells length to width ratio (Figure 22c). CMT1A rat primary Schwann cells displayed a significantly reduced length to width ratio compared to the WT controls independent of their stimulation (Figure 22d).

To test a functional outcome of morphology changes in CMT1A rat primary Schwann cells a Scratch assay was performed. Stimulated WT primary Schwann cells migrate significantly faster back in the Scratch than the unstimulated controls. CMT1A rat primary Schwann did not display any difference in migration speed after stimulation (Figure 23).

In summary, we can state that CMT1A primary Schwann cells differ in shape, polarity and migration behavior in vitro.

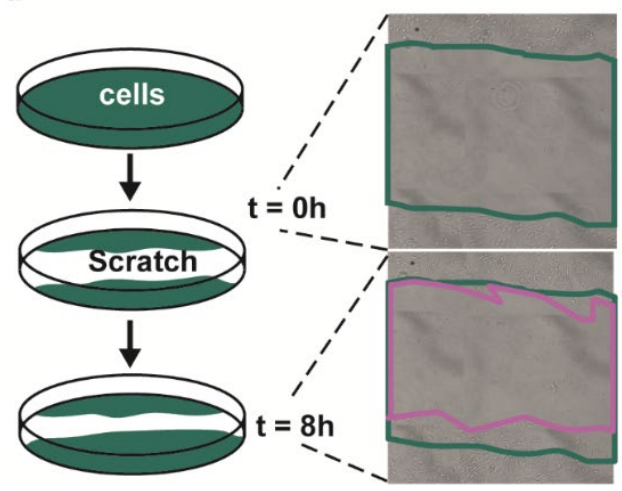

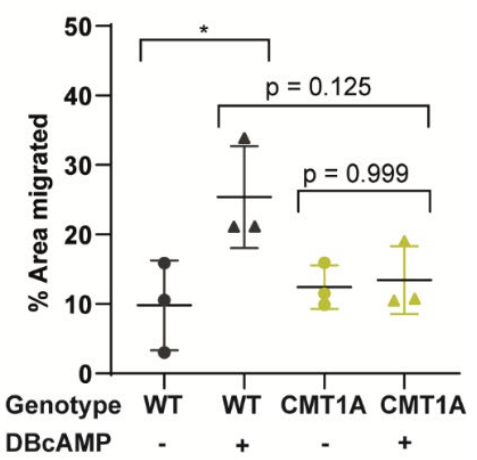

Figure 23: CMT1A primary Schwann cells migrate slower.

(a) Schematic overview of the Scratch Assay experiment. Primary Schwann cells were grown until confluent, scratched and observed for $8 \mathrm{~h}$.

(b) Quantification of Scratch Assays reveals WT primary Schwann cells migrate faster after stimulation (grey) while CMT1A Schwann cell do not increase migration speed upon stimulation (yellow). Per genotype and condition $\mathrm{n}=3$ biological replicates (primary Schwann cell preparations) with three technical replicates (Scratch Assays) were performed. Means are displayed \pm SD. Statistical analysis: One-way ANOVA with Sidak's multiple comparison test. ${ }^{*} \mathrm{p} \leq 0.05,{ }^{* *} \mathrm{p} \leq 0.01,{ }^{* * *} \mathrm{p} \leq 0.001$ and ${ }^{* * * *} \mathrm{p} \leq 0.0001$.

\subsubsection{CMT1A peripheral nerves display enlarged and disrupted Cajal bands.}

In the next step, we wanted to analyze whether Vinculin containing compartments are altered in CMT1A in vivo. As previously described, Vinculin is mainly expressed in paranodal loops and bands of Cajal in vivo.

Cajal bands are cytoplasmic channels on the abaxonal site of the myelin, which are located around adhesion contacts, so-called appositions, adhering the abaxonal Schwann cell plasma membrane to the compact myelin. Previous studies discovered a correlation between Cajal band integrity and internodal length in peripheral nerves (Court et al 2004, Court et al 2009). A typical hallmark of CMT1A is a reduction in internodal length (Saporta et al 2009, Fledrich et al 2014). Cajal band integrity has not been assessed in CMT1A in detail, yet. Therefore, we analyzed Cajal bands by immunofluorescence of teased fiber preparation and in sciatic nerve cross sections on electron microscopy level. 
Teased fiber preparations from CMT1A rats displayed disturbed Cajal bands, while appositions were visible, but smaller and less structured than in WT controls (Figure 24a). The f-ratio describes the ratio between Cajal band membrane and length of the appositions (Figure 24b). Axons in 4 weeks old CMT1A sciatic nerves displayed a clear shift towards fewer appositions. The majority of axons showed one or two appositions, while in the wildtype mainly two to three appositions were observed (Figure 24b-c). Accordingly, CMT1A nerves depicted a significantly increased f-ratio (Figure 24d-e).

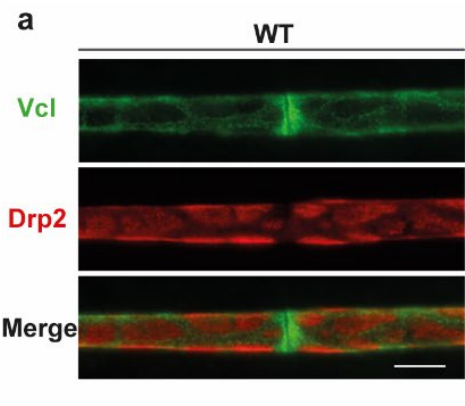

C

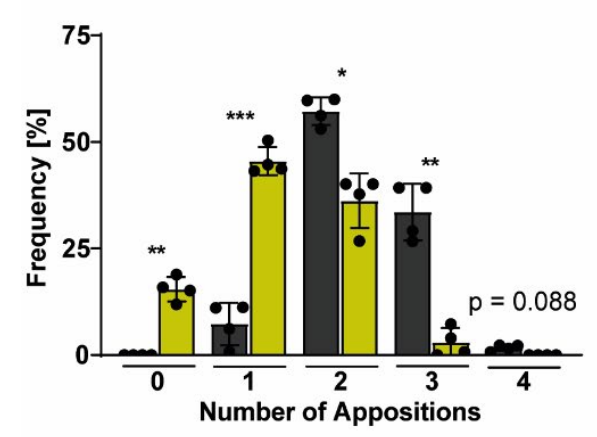

b

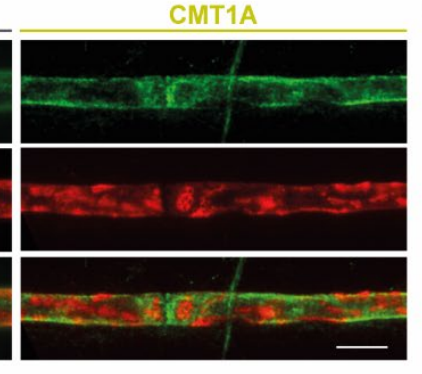

d

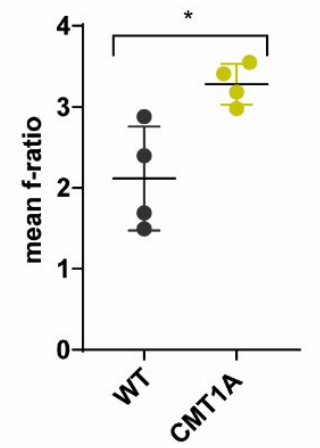

WT

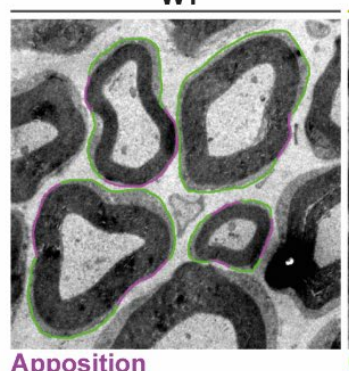

Apposition

e

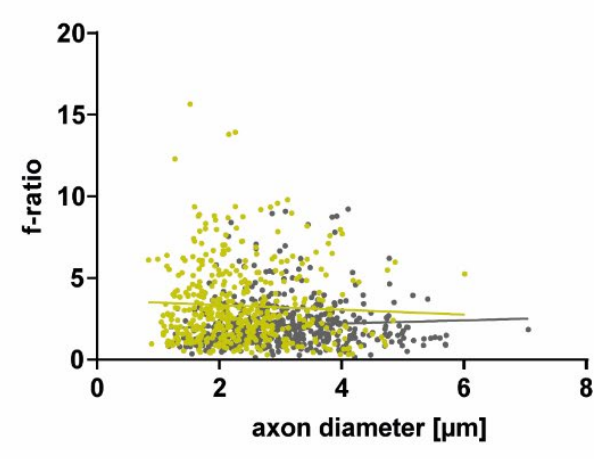

Figure 24: CMT1A nerves display less appositions and bigger Cajal band membranes.

(a) Immunofluorescent staining of WT and CMT1A teased fibers at 9 weeks of age display disrupted Cajal band (Vinculin, green) and Appositions (Drp2, red) in CMT1A (right panels). Scale bar is $10 \mu \mathrm{m}$.

(b) Electron micrograph images of wildtype (left) and CMT1A (right) rat sciatic nerves at postnatal day 28. Green indicates Cajal band membranes purple appositions. Robert Fledrich provided electron microscopy images.

(c) Quantification of the number of appositions per SC-axon unit shows fewer appositions in CMT1A (yellow) versus WT controls (grey).

(d) The f-ratio is calculated by dividing the length of the Cajal band membranes by the length of the appositions of one SC-axon unit. CMT1A nerves display an increased f-ratio, Cajal bands are bigger and appositions are smaller.

(e) Scatter plot distribution of f-ratio against axon diameter of WT (grey) and CMT1A (yellow) sciatic nerves.

Per genotype $\mathrm{n}=4$ biological replicates (animals) with each 130 technical replicates (axons) were quantified. Means are displayed \pm SD. Statistical analysis: Two-way ANOVA with Sidak's multiple comparison test (b); MannWhitney test $(\mathrm{d}) .{ }^{*} \mathrm{p} \leq 0.05,{ }^{* *} \mathrm{p} \leq 0.01,{ }^{* * *} \mathrm{p} \leq 0.001$ and ${ }^{* * *} \mathrm{p} \leq 0.0001$.

\subsubsection{Vinculin depletion in Schwann cells does not lead to a PTEN-associated phenotype.}

Cellular compartments in which Vinculin is present, displayed a disorganized phenotype in vitro and in vivo. According to our hypothesis that Vinculin is a direct or indirect interaction partner of PMP22, Vinculin might stabilize PTEN. Vinculin and PTEN are localized to Cajal bands in the Schwann cell in vivo (Figure 5, Figure 21). Vinculin null cells lack PTEN on protein 
level in vitro and re-transfection of Vinculin in these cells rescued PTEN protein levels (Subauste et al 2005). If this holds also true for Schwann cells, Vinculin depletion in Schwann cells will result in a phenotype similar to the PTEN knockout phenotype (Goebbels et al 2012).

\section{Generation of Schwann cell specific Vinculin knockout mice.}

Vinculin full knockout mice are lethal at embryonic state (Xu et al 1998). We made use of the Cre-loxP technology to study the hypothesis of Vinculin as a linker between PMP22 and PTEN mediated PI3K/Akt/mTOR signaling in the Schwann cell. Therefore, we crossbred Vinculin floxed mice [Zemljic-Harpf et al 2007] with Dhh-cre mice, which express a cre recombinase under the Dhh promotor to specifically delete the Vinculin gene only in Schwann cells (Figure 25a). PCR confirmed a recombination only in nerve tissue and not in Spinal cord, muscle and liver of Vinculin conditional knockout mice (VclcKO, Figure 25b). Primary Schwann cells of VclcKO mice did not show Vinculin immunoreactivity although focal adhesion plaques were present, as visualized by talin staining (Figure 25c).

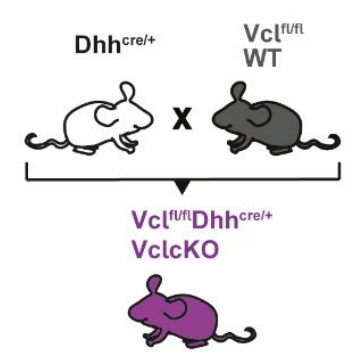

b

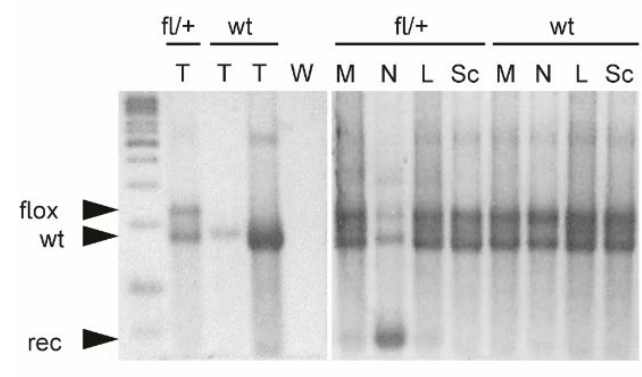

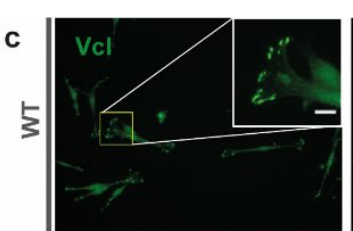
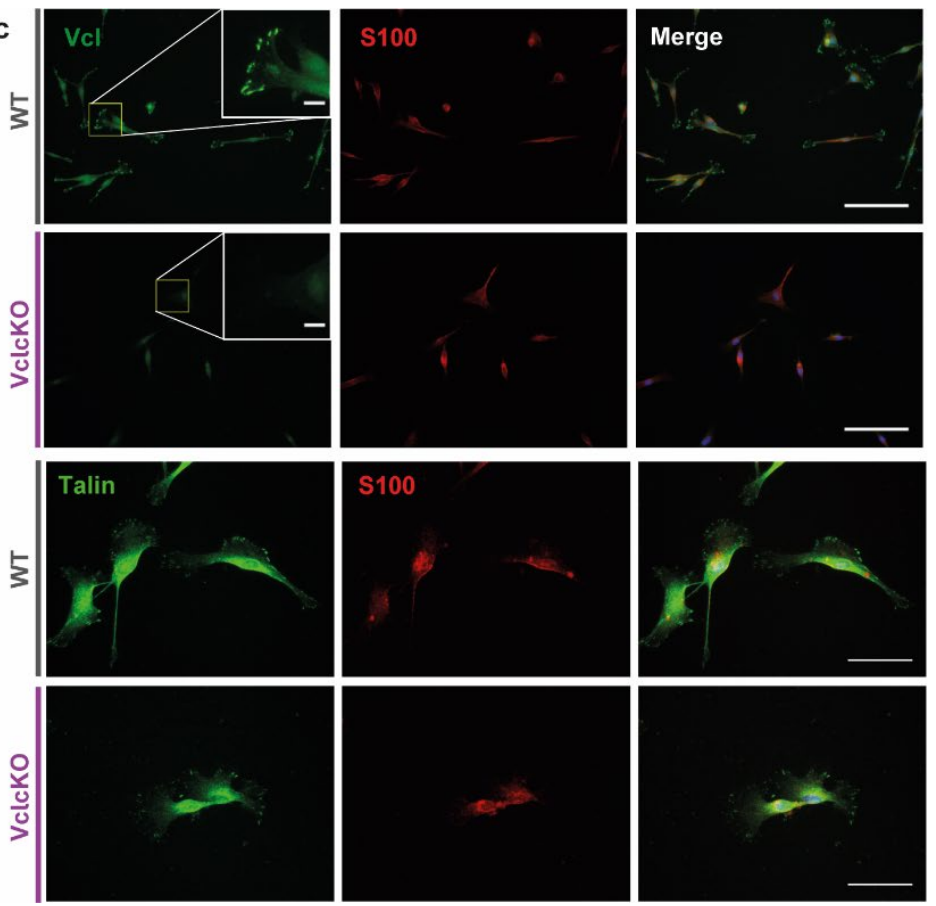

Figure 25: Generation of Schwann cell specific Vinculin knockout mice.

(a) Vinculin floxed mice were crossbred with Dhh-cre driver line to generate a Schwann cell specific deletion of Vinculin (VclcKO).

(b) PCR analysis confirms Vinuclin flox and wildtype band in fl/+ tails (T), while wildtype (wt) tails only display the wildtype band and the water (W) control no band. Tissue specific recombination is confirmed by using PCR using tissue from muscle (M), peripheral nerve (N), liver (L) and Spinal cord (Sc) of fl/+ and wt animals. Only peripheral nervous tissue from $\mathrm{fl} /+$ displays a recombination band at $800 \mathrm{bp}$.

(c) Mouse primary Schwann cells from WT animals reveal a localization of Vinculin (green) to focal adhesion plaques (upper row), whereas Vinculin immunoreactivity is lost in VclcKO mice (second row from top). S100 (red, second column) is used as a Schwann cell marker protein. Talin staining (green, third and fourth row) was used to show that focal adhesion plaques still form in VclcKO mice. 


\section{Vinculin depletion in Schwann cells does not influence myelination.}

If Vinculin stabilizes PTEN in Schwann cells, Vinculin conditional knockout mice (VclcKO) might mimic the PTEN conditional knockout phenotype, characterized by myelin outfoldings and tomacula formation. Cross section analysis of VclcKO femoral nerves displayed no obvious abnormalities and myelin thickness as well as axon diameter and axon number were indifferent in 8 weeks old animals (Figure 26a-e). To rule out a later effect of Vinculin deletion in Schwann cells, we analyzed 26 weeks old mice and also could not observe any changes in radial myelination (Figure $\mathbf{2 6 f - j}$ ).
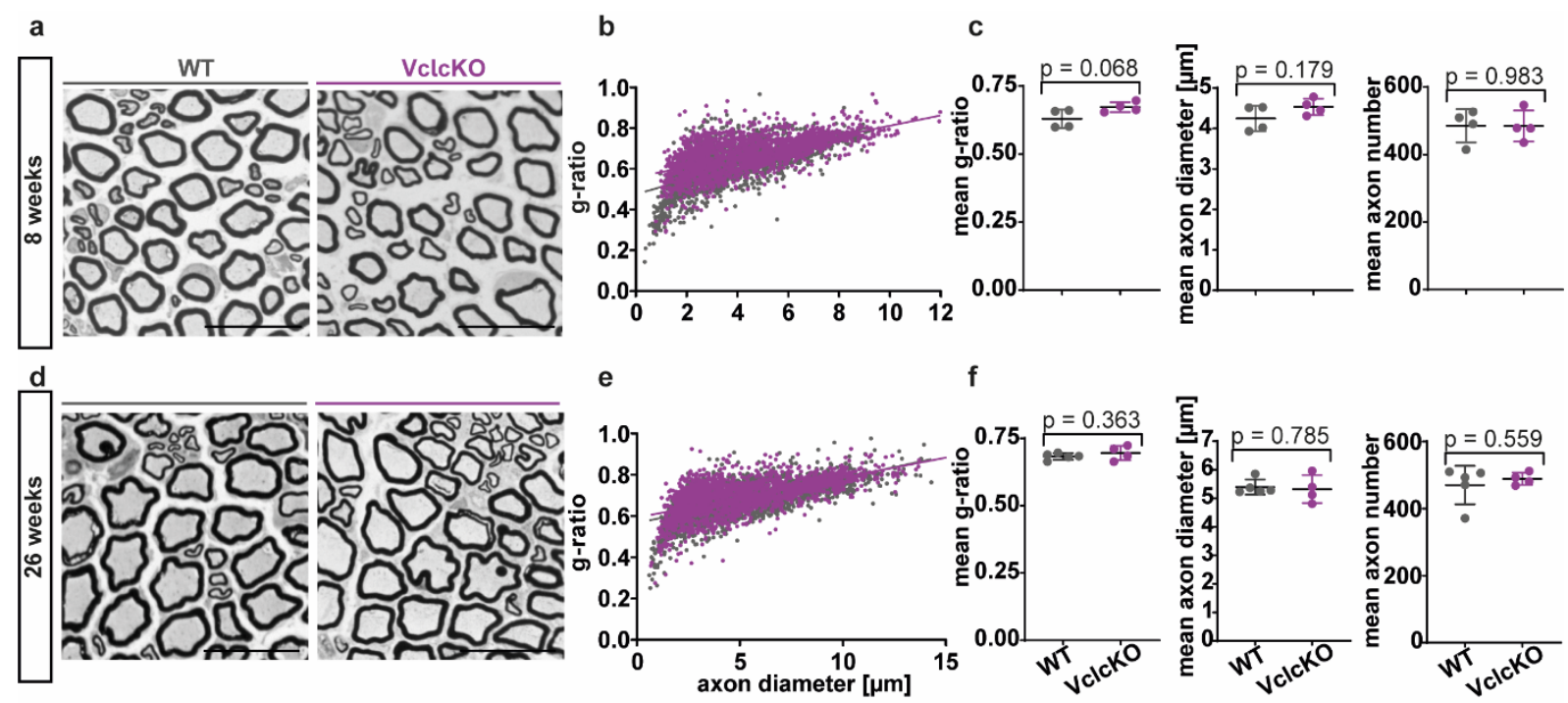

Figure 26: Radial myelination is unaltered in Vinculin conditional knockout mice.

(a) Example images of femoral nerve semi thin section of 8 weeks old WT (left) and VclcKO mice (right). Scale bar is $10 \mu \mathrm{m}$.

(b) Scatter plot displaying g-ratio versus axon diameter distribution of WT (grey) and VclcKO (purple) femoral nerves at 8 weeks of age.

(c) Mean g-ratio, axon diameter and axon number of WT (grey) and VclcKO (purple) mice was not significantly different in 8 weeks old femoral nerves.

(d) Example images of femoral nerve semi thin section of 26 weeks old WT (left) and VclcKO mice (right). Scale bar is $10 \mu \mathrm{m}$.

(e) Scatter plot displaying g-ratio versus axon diameter distribution of WT (grey) and VclcKO (purple) femoral nerves at 26 weeks of age.

(f) Mean g-ratio, axon diameter and axon number of WT (grey) and VclcKO (purple) mice was not significantly different in 26 weeks old femoral nerves.

Quantification was performed in $n=4 \mathrm{WT}$ and $\mathrm{n}=4 \mathrm{VclcKO}$ (8weeks) and $\mathrm{n}=5 \mathrm{WT}$ and $\mathrm{n}=4 \mathrm{VclcKO}$ whole femoral nerves. Means are displayed \pm SD. Statistical analysis: Unpaired student's test. ). ${ }^{*} p \leq 0.05,{ }^{* *} p \leq 0.01,{ }^{* * *} p \leq 0.001$ and ${ }^{* * * *} \mathrm{p} \leq 0.0001$.

\section{Vinculin depletion does not alter Cajal band and nodal integrity.}

Vinculin localizes to Cajal bands and paranodal loops in the Schwann cell in vivo. Therefore, we analyzed the impact of Vinculin depletion especially in these compartments. Staining of teased fibers revealed absence of Vinculin in Cajal bands, although the overall structure 
appeared unchanged (Figure 27a). Moreover, nodal as well as paranodal width was unaltered in Vinculin conditional knockout mice at 8 weeks of age (Figure $27 \mathbf{b}-\mathbf{d}$ ).

a
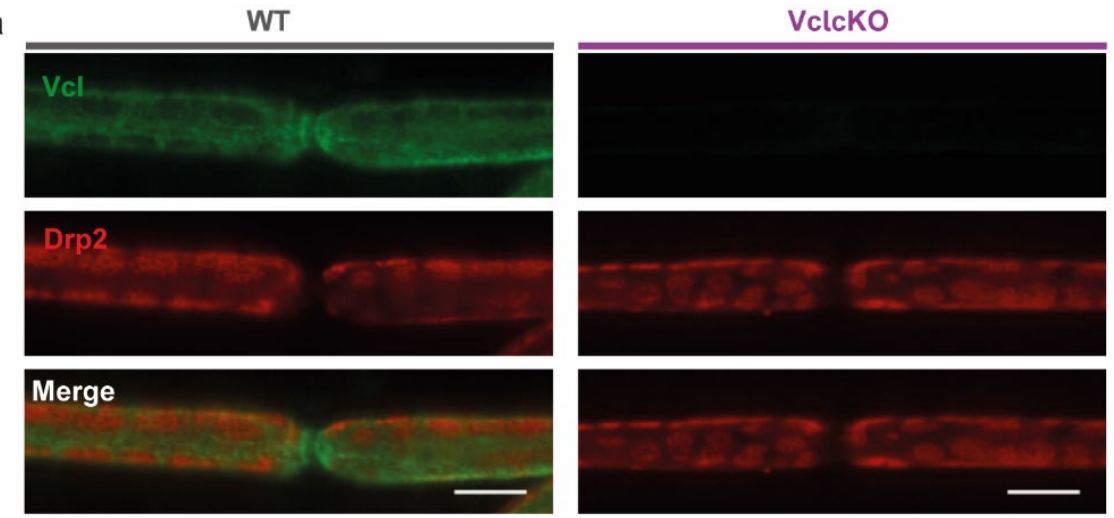

b
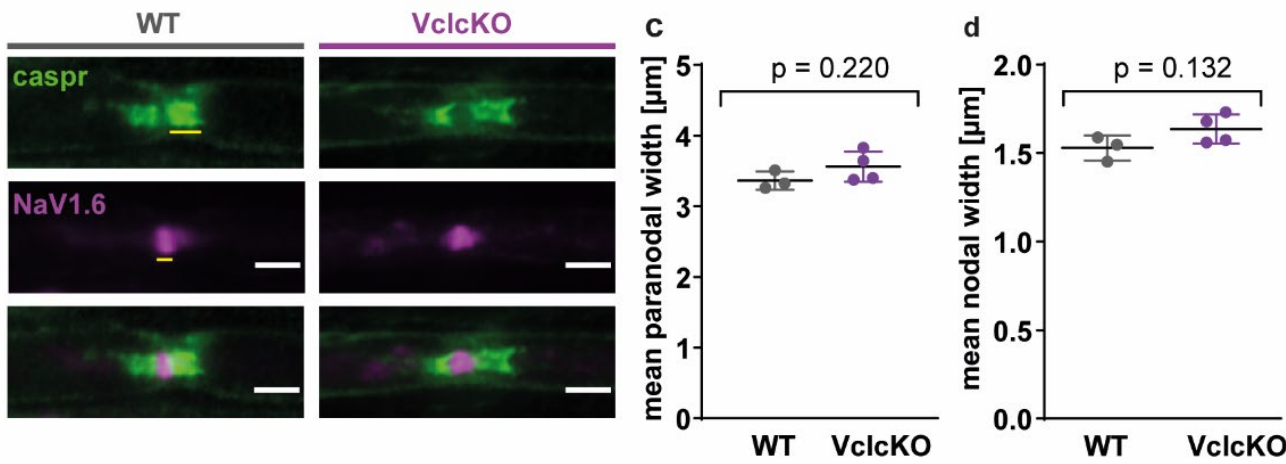

Figure 27: Vinculin depletion does not change Cajal band and nodal integrity.

(a) Teased fiber preparations show diminished Vinculin signal (green) from 8 weeks old VclcKO mice (second column), while Appositions (Drp2, red) appeared normal at 8 weeks of age.

(b) Analysis of nodal compartment by staining teased fibers to visualize paranodes (green, caspr) and nodes (red, NaV1.6). Yellow lines indicate the applied measurements of paranodal and nodal width at 8 weeks.

(c) Vinculin conditional knockout mice (purple) displayed no difference in paranodal width compared to WT controls (grey) at 8 weeks of age.

(d) Vinculin depletion in Schwann cells did not change mean nodal width.

Means are displayed $\pm S D, n=3 W T$ and $n=4$ VclcKO were analyzed. For each animal 100 nodes were quantified. Statistical analysis: Unpaired student's t-test. ${ }^{*} \mathrm{p} \leq 0.05,{ }^{* *} \mathrm{p} \leq 0.01,{ }^{* * *} \mathrm{p} \leq 0.001$ and ${ }^{* * * *} \mathrm{p} \leq 0.0001$

\section{Vinculin expression does not alter PTEN protein levels in vitro and in vivo.}

Molecular analysis of proteins in sciatic nerve lysates revealed a significant reduction of Vinculin (Figure 28a,b), while residual amounts might be attributed to Vinculin from the axon and connective tissue. Moreover, talin, a focal adhesion protein known as a binding partner of Vinculin (Burridge and Mangeat 1984), did not change in protein abundance in VclcKO mice (Figure 28a,b). Furthermore PMP22 protein levels were unaffected by Vinculin depletion in vivo as well as abundance of compact myelin protein P0 (Figure 28a,b), a known binding partner of PMP22 (Hasse et al 2004).

Thus, in contrast to carcinoma cells (Subauste et al 2005), endogenous levels of Vinculin in Schwann cells do not contribute to PTEN stabilization in vivo. As PMP22 is overexpressed in CMT1A and we observed an upregulation of PTEN and Vinculin on protein level, we assessed whether additional Vinculin can have any impact on PTEN levels and overexpressed Vinculin in HEK cells. Overexpression of wildtype Vinculin (Vcl-eGFP) and Vinculin containing a 
a

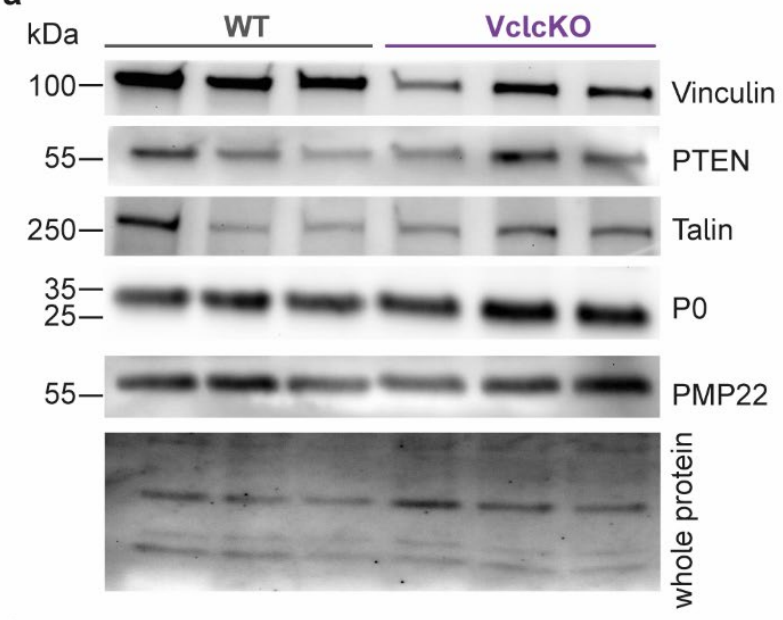

C

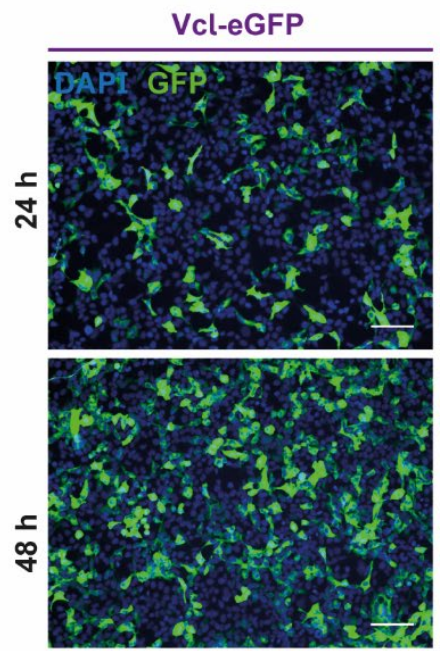

b

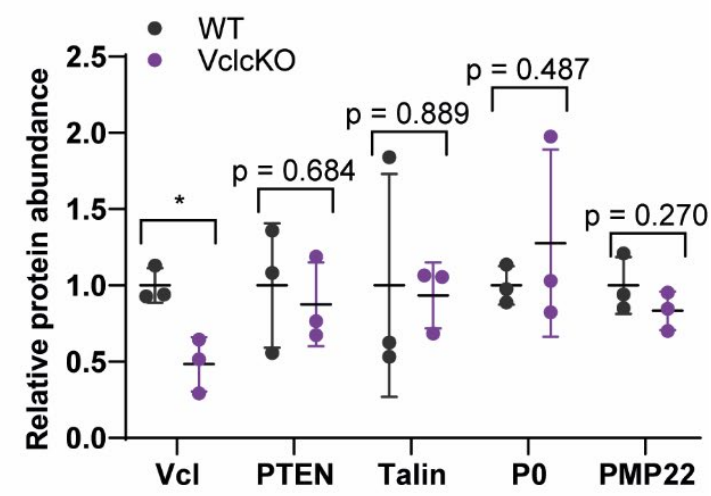

d

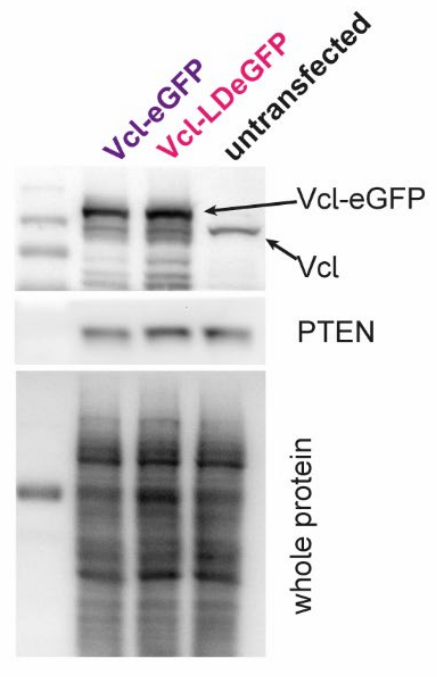

Figure 28: Vinculin expression does not influence PTEN protein level.

(a) Immunoblot analysis of Vinculin, PTEN, Talin, P0 and PMP22 in postnatal day 6 whole sciatic nerve lysates of WT and VclcKO mice. Fast green whole protein staining was used as loading controls.

(b) Quantification of (a) revealed significantly less Vinculin in VclcKO nerves and unchanged protein levels of PTEN, Talin, P0 and PMP22.

(c) Transfection of HEK cells with either Vcl-eGFP or Vcl-LDeGFP showed good transfection efficacy after 48 hours.

(d) Immunoblot analysis of HEK transfected cells after $48 \mathrm{~h}$ (c) displayed unchanged PTEN protein level. Fast green whole protein staining was used as loading controls. Plasmids were kindly provided by Johannes Hirrlinger.

Means are displayed \pm SD. For each genotype nerves of $n=3$ animals were analyzed. Statistical analysis: Multiple ttest. ${ }^{*} \mathrm{p} \leq 0.05,{ }^{* *} \mathrm{p} \leq 0.01,{ }^{* * *} \mathrm{p} \leq 0.001$ and ${ }^{* * * *} \mathrm{p} \leq 0.0001$.

mutated $\mathrm{PIP}_{2}$ binding site in HEK cells did not alter PTEN protein level in vitro (Figure $\mathbf{2 8 c}$,d), in agreement with our data on Vinculin conditional knockout in vivo. Since on different cellular backgrounds, neither depletion nor overexpression of Vinculin had any influence on PTEN, we reject the hypothesis that PTEN stabilization upon PMP22 overexpression is mediated by Vinculin. Thus, it remains open how PMP22 influences PTEN level and the downstream PI3K/AKT/mTOR signaling pathway in CMT1A and HNPP. 


\subsection{Modulating Schmidt-Lanterman incisure number in CMT1A.}

We generated Schwann cell specific Vinculin conditional knockout mice to get further insight in possible interaction partners of PMP22 and implications for growth signaling in CMT1A (4.3). However, we could not show a connection between Vinculin and PTEN in the Schwann cell. Nevertheless, Vinculin depleted mice revealed a role for Vinculin in longitudinal myelination.

\subsubsection{Vinculin conditional knockout mice display reduced numbers of Schmidt- Lanterman incisures.}

Staining teased fibers of Vinculin depleted mice using MAG as a marker of uncompacted myelin (paranodal loops, Schmidt-Lanterman Incisures) and NaV1.6 to visualize nodes of Ranvier allows to evaluate Schmidt-Lanterman Incisures and to determine internodal length (Figure 29a). Interestingly, 8 weeks old Vinculin conditional knockout mice revealed unaltered

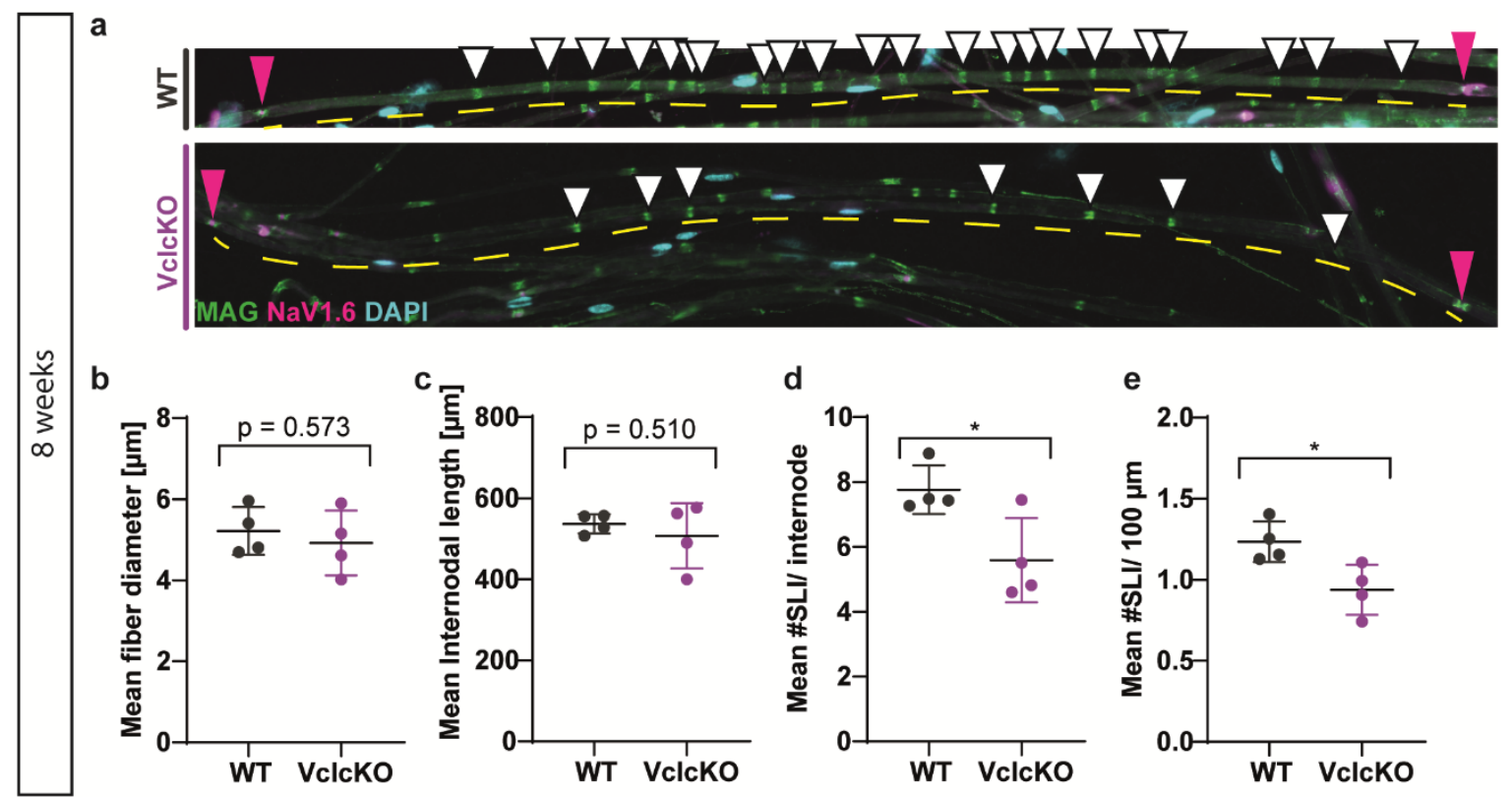

Figure 29: Teased fiber preparations of 8 weeks old VclcKO mice display reduced numbers of SchmidtLanterman incisures.

(a) Example immunofluorescence images of WT (grey, upper panel) and VclcKO (purple, lower panel) teased fiber preparations from sciatic nerves at 8 weeks of age. Two nodes of Ranvier (NaV1.6, magenta arrowheads) flank one internode (yellow, dashed lines). MAG stains uncompacted regions of the Schwann cell, such as SLIs (white arrowheads). DAPI (cyan) is a marker for Schwann cell nuclei. Teased fibers from VclcKO nerves (lower panel) display a decrease in SLIs.

(b) WT nerves show no significant change in fiber diameter compared to VclcKO nerves.

(c) Internodal length is not different in WT and VclcKO nerves.

(d) The number is SLI per internode significantly decreased in VclcKO nerves.

(e) Quantification of SLI number per $100 \mu \mathrm{m}$ reveals a significant reduction in VclcKO nerves.

Quantification was performed in sciatic nerve teased fiber preparations from $n=4$ animals per genotype, while 100 internodes per animal were analyzed. Statistical analysis: unpaired student's t-test ${ }^{*} p \leq 0.05,{ }^{* *} p \leq 0.01,{ }^{* * *} p \leq 0.001$ and ${ }^{* * * *} \mathrm{p} \leq 0.0001$. 
internodal length and fiber diameter while the number of Schmidt-Lanterman incisures decreased by $\sim 30 \%$ (Figure 29b-e).

Schmidt-Lanterman Incisures are cytoplasmic channels in the compact myelin. Schmidt and Lanterman described these prominent structures already in 1874 and 1877, respectively, but their function remains speculative.

We wondered whether Schmidt-Lanterman formation impairment is already visible early in development in Vinculin conditional knockout mice and thus assessed teased fiber preparations from 6 days old mice. Wildtype teased fibers already displayed typical funnelshaped incisures at this early time point while VclcKO fibers showed dense MAG staining at the abaxonal membrane but hardly any fully developed SLIs (Figure 30a). Quantification revealed no change in fiber diameter and internodal length at postnatal day 6, however Schmidt-Lanterman formation lags behind in Vinculin depleted Schwann cells (Figure 30b-e).
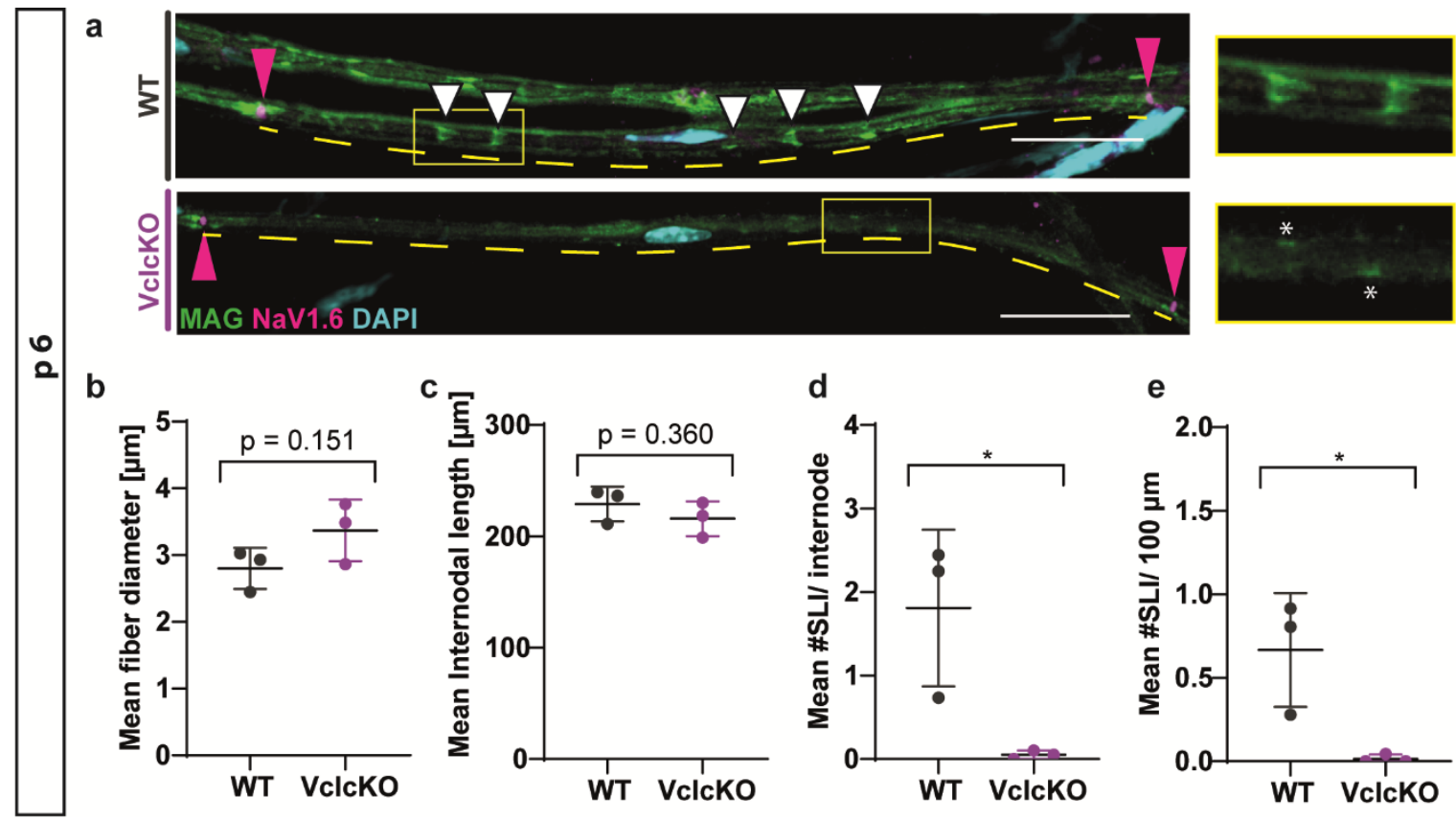

b

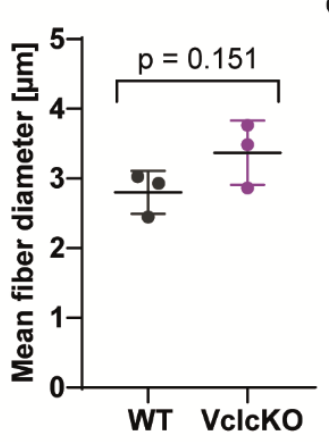

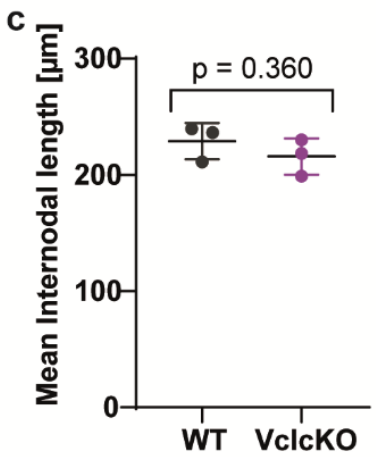
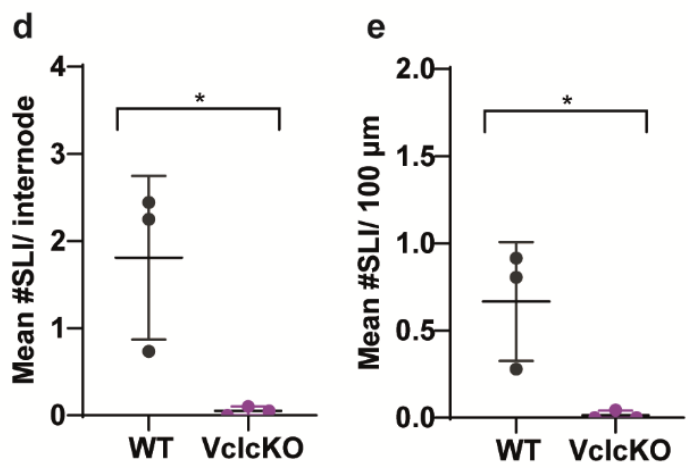

Figure 30: Teased fiber preparations of 6 days old VclcKO mice display reduced numbers of Schmidt-Lanterman incisures.

(a) Example immunofluorescence images of WT (grey, upper panel) and VclcKO (purple, lower panel) teased fiber preparations from sciatic nerves at postnatal day 6. Two nodes of Ranvier (NaV1.6, magenta arrowheads) flank one internode (yellow, dashed lines). MAG stains uncompacted regions of the Schwann cell, such as SLIs (white arrowheads). DAPI (cyan) is a marker for Schwann cell nuclei. Close up images (yellow rectangle, right column) show consistent MAG staining in the WT (upper panel), while the VclcKO (lower panel) does not display complete SLIs, although some more intense MAG staining is visible abaxonally, indicate by white stars. Scale bar is $20 \mu \mathrm{m}$.

(b) WT nerves show no significant change in fiber diameter compared to VclcKO nerves.

(c) Internodal length is not different in WT and VclcKO nerves.

(d) The number is SLI per internode significantly decreased in VclcKO nerves.

(e) Quantification of SLI number per $100 \mu \mathrm{m}$ reveals a significant reduction in VclcKO nerves.

Quantification was performed in sciatic nerve teased fiber preparations from $n=3$ animals per genotype, while 3050 internodes per animal were analyzed. Statistical analysis: unpaired student's t-test ${ }^{*} p \leq 0.05,{ }^{* *} p \leq 0.01,{ }^{* * *} p \leq$ 0.001 and $^{* * * *} \mathrm{p} \leq 0.0001$. 


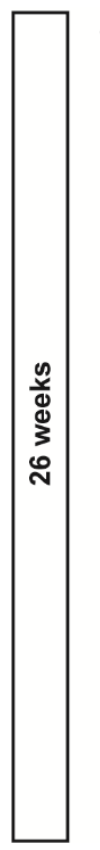

a
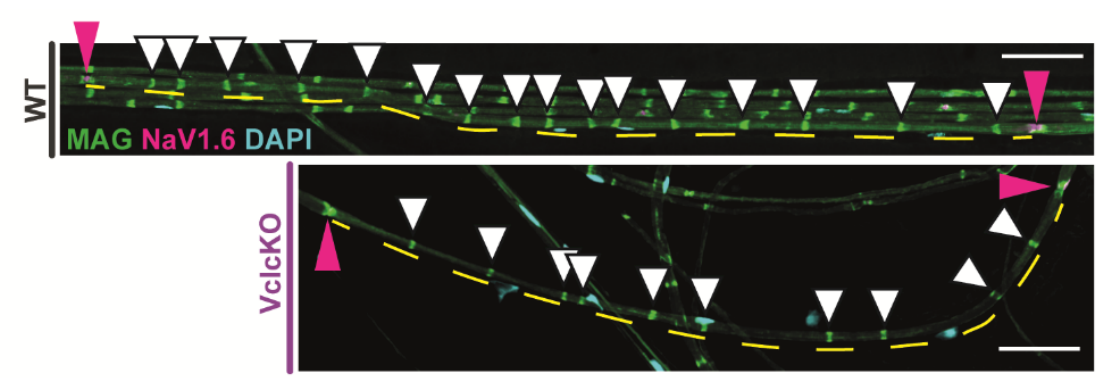

b

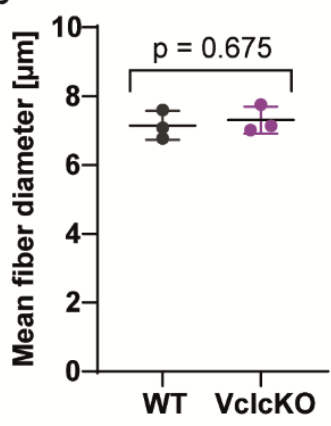

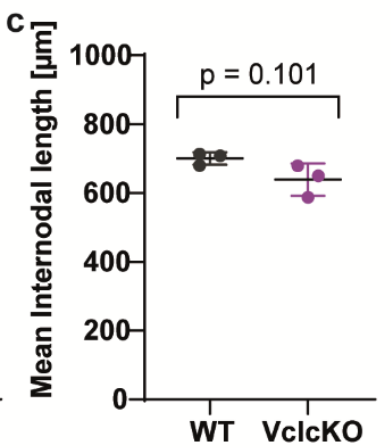
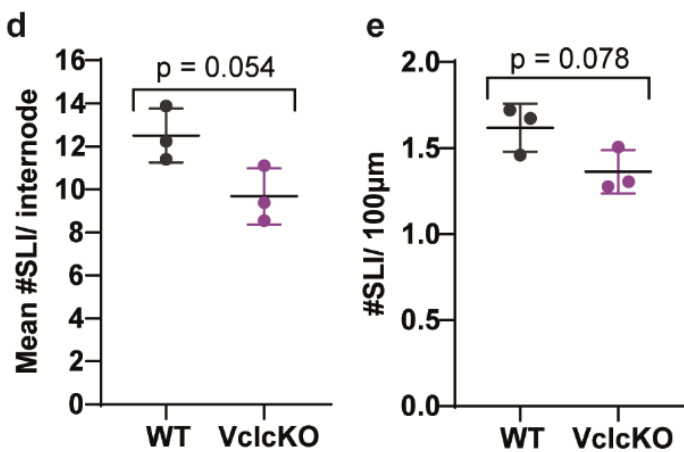

Figure 31: Teased fiber preparations of 26 weeks old VclcKO mice display reduced numbers of SchmidtLanterman incisures.

(a) Example immunofluorescence images of WT (grey, upper panel) and VclcKO (purple, lower panel) teased fiber preparations from sciatic nerves at 26 weeks of age. Two nodes of Ranvier (NaV1.6, magenta arrowheads) flank one internode (yellow, dashed lines). MAG stains uncompacted regions of the Schwann cell, such as SLIs (white arrowheads). DAPI (cyan) is a marker for Schwann cell nuclei. Teased fibers from VclcKO nerves (lower panel) display a decrease in SLIs compared to wildtype controls (upper panel). Scale bar is $50 \mu \mathrm{m}$.

(b) WT nerves show no significant change in fiber diameter compared to VclcKO nerves.

(c) Internodal length is not different in WT and VclcKO nerves.

(d) The number is SLI per internode significantly decreased in VclcKO nerves.

(e) Quantification of SLI number per $100 \mu \mathrm{m}$ reveals a significant reduction in VclcKO nerves.

Quantification was performed in sciatic nerve teased fiber preparations from $n=3$ animals per genotype, while 100 internodes per animal were analyzed. Statistical analysis: unpaired student's t-test, ${ }^{*} \mathrm{p} \leq 0.05,{ }^{* *} \mathrm{p} \leq 0.01,{ }^{* * *} \mathrm{p} \leq 0.001$ and ${ }^{* * * *} \mathrm{p} \leq 0.0001$.

These observations raised the question, whether SLI number, which is reduced by $\sim 25 \%$ in 8 weeks old mice, catches up in older animals. Therefore, we analyzed 26 weeks old mice and still observed a $\sim 25 \%$ reduction in SLIs, while fiber diameter and internodal length did not change (Figure 31). Hence, Vinculin depletion in Schwann cells partially impairs SLI formation, while leaving other aspects of myelination unaffected.

In shiverer (MBP null mutants) SLI number is increased (Gould et al 1995). This holds true for Cerebroside sulfotransferase deficient mice as well (Hoshi et al 2007). Schmidt-Lanterman incisure formation has been reported to be vanished in myelin protein P0 and Periaxin null mutants (Yin et al 2008, Sherman et al 2012). To our knowledge, a decrease of SchmidtLanterman incisures without affecting compact myelin formation has not been reported, yet. Therefore, Vinculin conditional knockout mice can provide a useful model to study SchmidtLanterman incisure function in the peripheral nervous system.

Different functions are proposed for Schmidt-Lanterman incisures in the literature. As SLIs form cytoplasmic channels through the compact myelin sheath, they might function as fast radial transport routes for metabolites and nutrients from the Schwann cell to the axon. Moreover, membrane degradation and addition are thought to take place at the site of SLIs 
during nerve injury and myelin maintenance. SLIs can be seen as elastic regions which provide mechanical flexibility in the nerve.

\subsubsection{Depletion of Vinculin impairs the CMT1A phenotype.}

As previously mentioned, animals models of neuropathies were reported with changes in SLI number. This holds true for the CMT1A rat and CMT1A mouse models, which display increased numbers of SLIs. Vinculin conditional knockout mice have a reduction in SLI number but an otherwise unobtrusive phenotype. We asked the question whether Vinculin depletion reduces SLI numbers in CMT1A nerves and whether this impacts the behavioral and

a

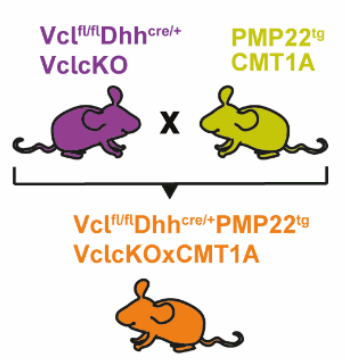

b
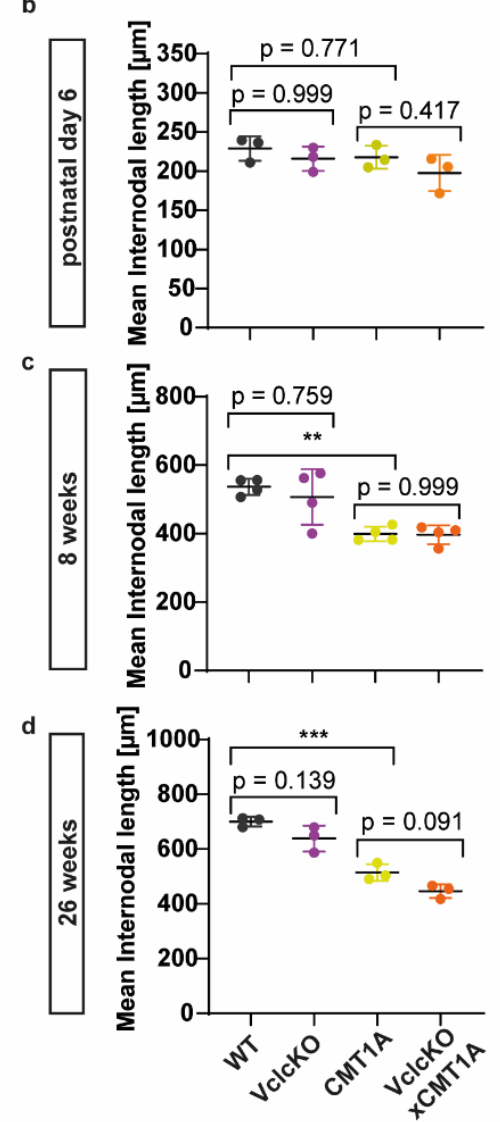
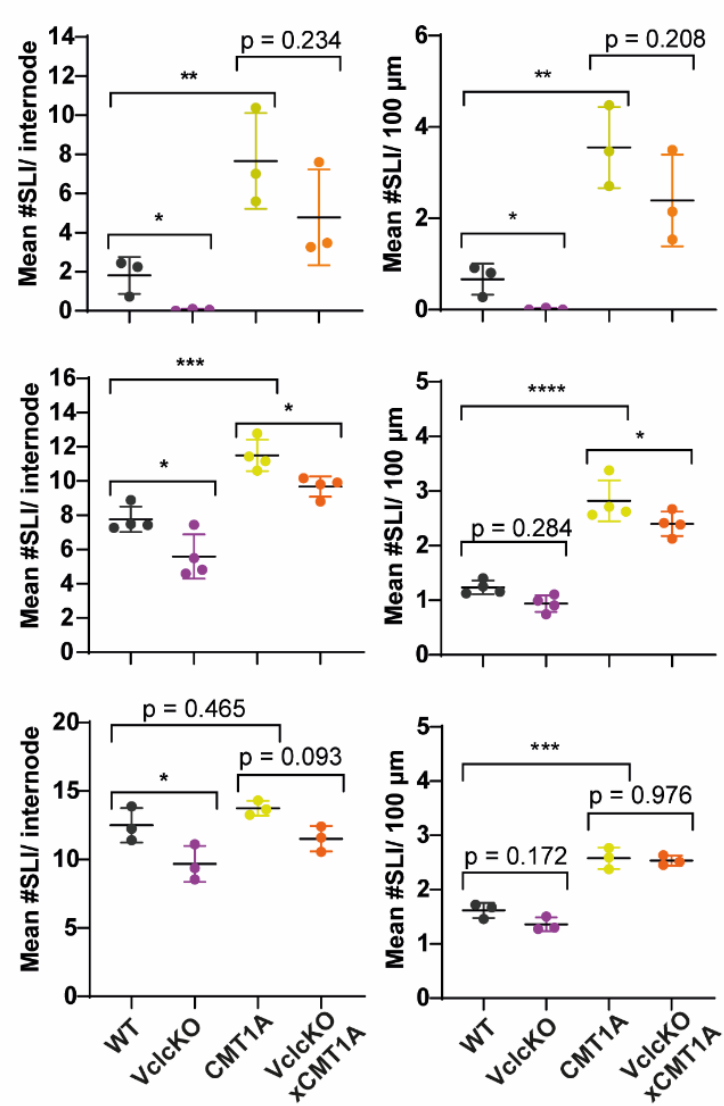

Figure 32: Vinculin depletion in CMT1A Schwann cells decreases the number of Schmidt-Lanterman Incisures.

(a) Crossbreeding VclcKO mice with CMT1A mice generated VclcKOxCMT1A mice.

(b) At postnatal day 6 no difference in internodal length is observed between the four groups (left panel). However, VclcKOxCMT1A double mutants (orange) display a tendency towards decreased number of SLI compared to CMT1A mice (yellow), which show significantly more SLI (middle, right panel).

(c) At 8 weeks of age, internodes in CMT1A mice are significantly shorter than WT controls, while VclcKOxCMT1A internodes are indifferent from CMT1A (left panel). The number of SLI is significantly increased in CMT1A mice compared to WT controls, whereas VclcKOxCMT1A mice display a significant reduction in SLI (middle, right panel).

(d) At 26 weeks, CMT1A internodes are significantly shorter than in the WT controls, while VclcKOxCMT1A double mutants show a tendency towards reduced internodes at this time point (left panel). Besides, the number of SLI per internode tends to be lower in VclcKOxCMT1A compared to CMT1A mice (middle panel), whereas no difference is observed in the number of SLIs per $100 \mu \mathrm{m}$ (right panel).

Each data point represents one animal. At p6 30 and at 8 and 26 weeks 100 internodes were quantified per animal. Means are displayed \pm SD. Statistical analysis: One-way ANOVA with Sidak's multiple comparison test. ${ }^{*} \mathrm{p} \leq 0.05$, ${ }^{* *} \mathrm{p} \leq 0.01,{ }^{* * *} \mathrm{p} \leq 0.001$ and ${ }^{* * * *} \mathrm{p} \leq 0.0001$. 
electrophysiological phenotype of CMT1A mice. Therefore, we crossbred CMT1A mice with Vinculin Schwann cell specific knockout mice (Figure 32a).

We analyzed SLI number at postnatal day 6, 8 weeks and 26 weeks of age in the VclcKOxCMT1A double mutants. At all time points, CMT1A nerves revealed an increase in SLI number compared to wildtype controls with a reduction in internodal length (Figure 32). Vinculin depletion in CMT1A Schwann cells reduced Schmidt-Lanterman incisure number approximately in the same range as they were reduced in Vinculin conditional knockout mice compared to wildtype controls (Figure 32). Therefore, Vinculin depleted CMT1A mice did not completely lose the ability to increase SLI, but they did not show as many as visible in the CMT1A mouse. Thereby, internodal length appeared unchanged at postnatal day 6 and 8 weeks (Figure 32b,c), while double mutants show tendencies towards shorter internodes in older animals at 26 weeks of age (Figure 32d).

\section{Vinculin depletion in CMT1A leads to increased numbers of amyelinated axons.}

In the next step we aimed at analyzing the effect of reduced SLI number in CMT1A mice on radial myelination. Femoral nerves of 8 weeks old VclcKOxCMT1A mice displayed no significant alterations in mean g-ratio, axon diameter and axon number (Figure 33a,b), while the amount of amyelinated axons was significantly increased in the double mutants (Figure 33b). Although the number of amyelinated axons was increased in the double mutants, the scatter plot depiction of myelinated axons matched the one in CMT1A mice, indicating myelinated axons do not differ with Vinculin depletion (Figure 33c). Additionally, 26 weeks old femoral nerves of VclcKOxCMT1A double mutants displayed no changes in the mean $\mathrm{g}$ ratio whereas axon diameters were smaller and axon number tended to be reduced as well (Figure 33d-f). As described in the 8 weeks old double mutants, the percentage of amyelinated axons is significantly increased in Vinculin depleted CMT1A nerves (Figure 33e).

Taken together, Vinculin depletion in CMT1A nerves reduced SLI number and increased the amount of amyelinated axons.

VclcKOxCMT1A mice show a deteriorated motor phenotype compared to CMT1A mice.

It is hypothesized, that Schmidt-Lanterman Incisures provide shortcuts for metabolites from the Schwann cell to the axon and during acute (injury) and chronic (neuropathy) stress of the axon, the number of SLIs increases. Therefore, we tested whether a reduction of SLIs in VclcKOxCMT1A mice influences the behavioral and electrophysiological phenotype which could be a first prove for SLI as supporting routes from the Schwann cell to the axon.

We evaluated motor behavior of mice by analyzing fore- and hindlimb grip strength as well as slips and time on the elevated beam. To assess the sensory phenotype of the mice, we measured the reaction time on a $55{ }^{\circ} \mathrm{C}$ hot plate (Figure 34a).

The weight of the animals, as a measure of general health, was indifferent in all genotypes (Figure 34b). In addition, VclcKO mice did not show any motor or sensory impairments (Figure 34c-e). 

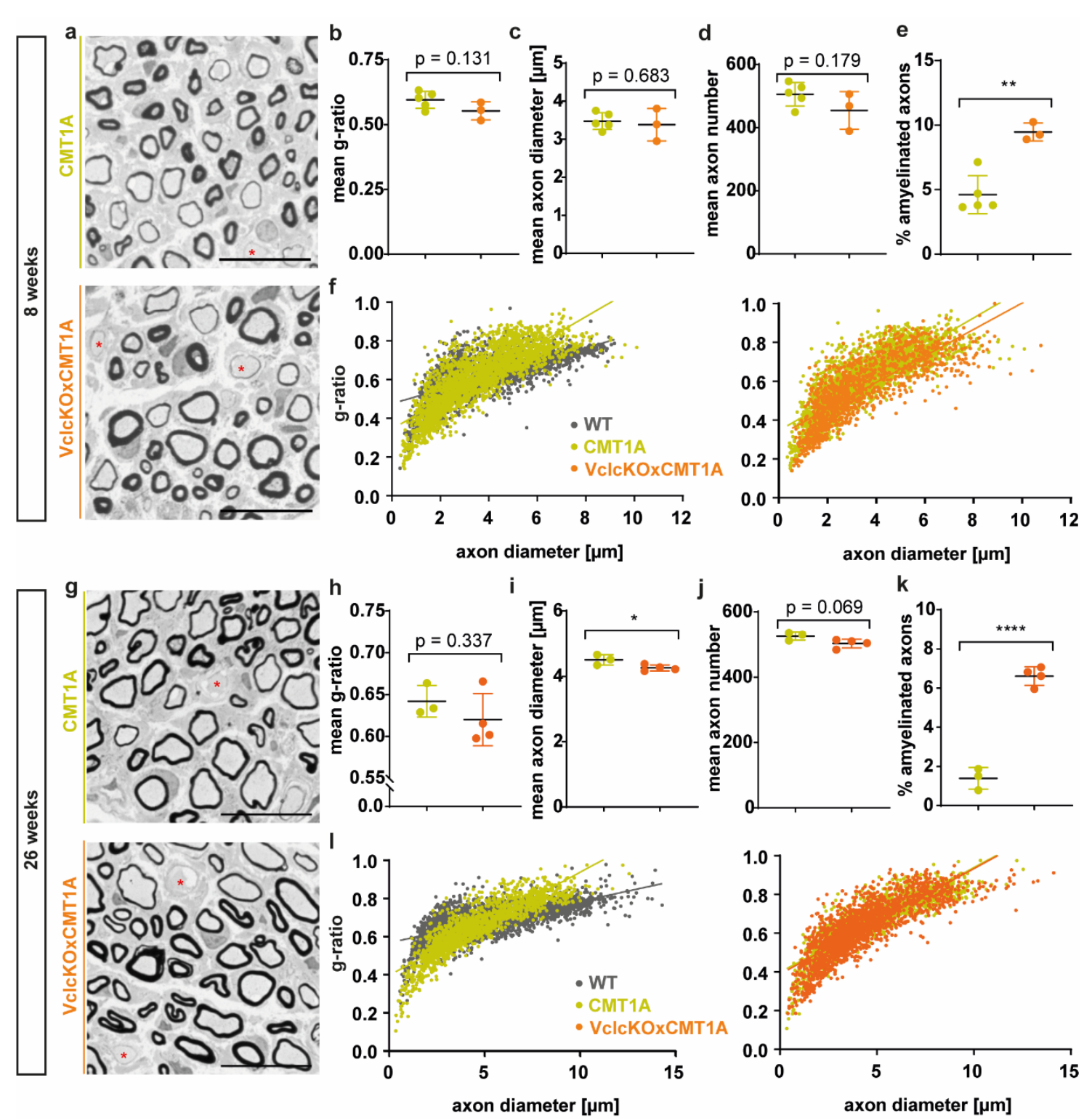

Figure 33: Vinculin depletion in CMT1A increases the number of amyelinated axons.

(a) Example images of semi-thin sections of CMT1A (upper panel) and VclcKOxCMT1A (lower panel) 8 weeks old femoral nerves. Stars indicate amyelinated axons. Scale bar is $20 \mu \mathrm{m}$.

(b) VclcKOxCMT1A mean g-ratio, axon diameter and axon number were not altered in 8 weeks old femoral nerves compared to CMT1A mice, while VclcKOxCMT1A double mutants displayed significantly more amyelinated axons.

(c) Scatter plot depiction of g-ratio versus axon diameter revels a shift showing more hyper- and hypomyelinated axons in CMT1A mice compared to WT (left panel) whereas VclcKOxCMT1A mice overlay very well with CMT1A mice (right panel, amyelinated axons not included).

(d) Example images of semi-thin sections of CMT1A (upper panel) and VclcKOxCMT1A (lower panel) 26 weeks old femoral nerves. Stars indicate amyelinated axons. Scale bar is $20 \mu \mathrm{m}$.

(e) Mean g-ratio was unchanged in 26 weeks old VclcKOxCMT1A mice, while axon diameter was significantly smaller. Moreover, the amount of amyelinated axons is significantly increased and double mutants tend to have less axons.

(f) Scatter plot depiction of g-ratio versus axon diameter revels a shift showing more hyper- and hypomyelinated axons in CMT1A mice compared to WT (left panel) whereas VclcKOxCMT1A mice overlay very well with CMT1A mice (right panel, amyelinated axons not included).

Means are displayed \pm SD $n=5$ CMT1A and $n=3$ VclcKOxCMT1A mice were analyzed in the 8 weeks time point whereas $n=3$ CMT1A and $n=4$ VclcKOxCMT1A were analyzed in the 26 weeks time point. One data point represents one animal. (b, e). In Scatterplots (c, f), one data point is one axon. Statistical analysis: Unpaired student's t-test. ${ }^{*} \mathrm{p} \leq 0.05,{ }^{* *} \mathrm{p} \leq 0.01,{ }^{* * *} \mathrm{p} \leq 0.001$ and ${ }^{* * *} \mathrm{p} \leq 0.0001$. 
On the elevated beam CMT1A mice slipped significantly more often and needed longer to balance $80 \mathrm{~cm}$, while VclcKOxCMT1A mice performed even worse (Figure 34d). Moreover, VclcKOxCMT1A mice displayed reduced force in hind- and forelimbs, while the effect is specifically prominent in the more distal hindlimbs, which displayed a stronger decrease in CMT1A mice as well (e). The sensory reaction on the hotplate was unaffected in all groups (Figure 34c).

a
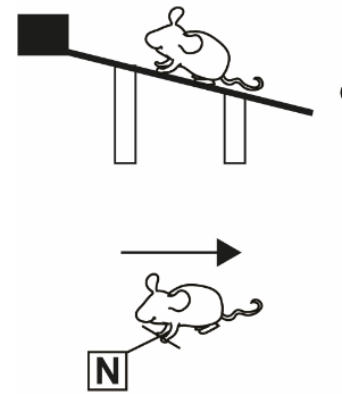
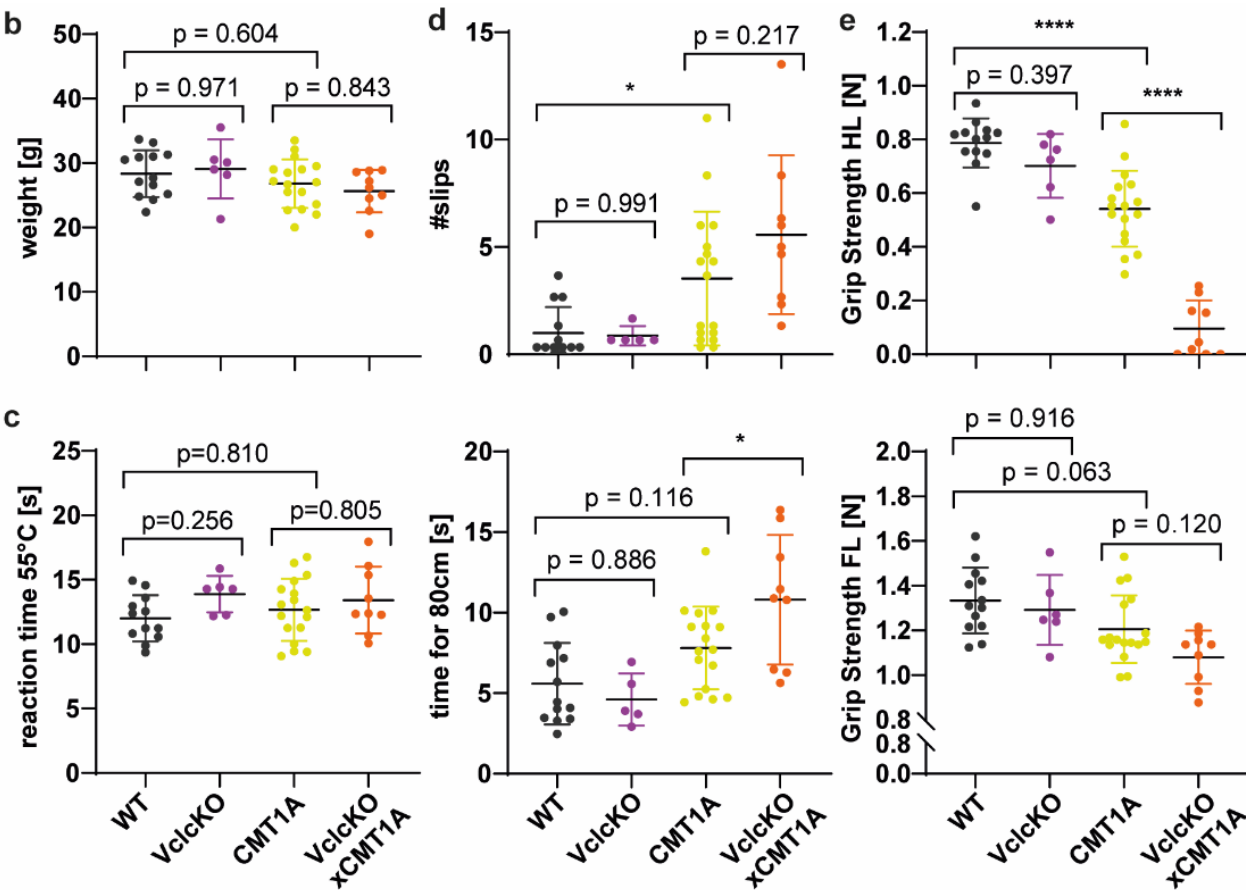

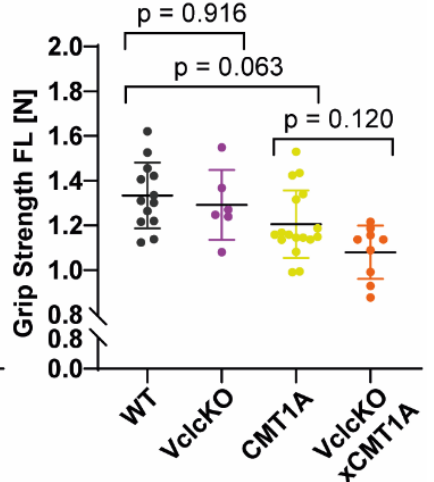

Figure 34: VclcKOxCMT1A mice display an impaired motor behavior.

(a) Mice went through three different behavioral tasks to study motor coordination and sensory characteristics. The hotplate test (upper sketch) examines the sensory reaction of the mice, the elevated beam test (middle sketch) serve to test motor coordination and we measured hind and forelimb grip strength analysis (lower sketch). (Detailed description see Material and Methods section 3.1.3).

(b) Weight is unaffected by genotype.

(c) Hotplate analysis reveals no change between all four groups.

(d) WT (grey) and VclcKO (purple) mice perform equally in the elevated beam test, while CMT1A mice (yellow) display more slips (upper panel) and tend to be slower (lower panel). When CMT1A mice additionally lack Vinculin in Schwann cells (orange), they are even slower (lower panel) and tend to slip more often (upper panel).

(e) CMT1A mice (yellow) display a strong reduction in hind limb grip strength (upper panel) compared to the wildtype controls (grey), whereas VclcKO mice (purple) do not differ. Moreover, VclcKOxCMT1A double mutants (orange) show an even stronger decrease in hind limb grip strength than the CMT1A mice. The effects observed in hind limb grip strength are not that prominent in assessing the forelimb grip strength (lower panel) but show a similar tendency.

Means are displayed \pm SD. WT $n=13$, VclcKO $n=6$, CMT1A $n=17$, VclcKOxCMT1A $n=9$ mice were analyzed at 16 weeks of age. Statistical analysis: One-way ANOVA with Sidak's multiple comparison test. ${ }^{*} \mathrm{p} \leq 0.05,{ }^{* *} \mathrm{p} \leq 0.01$, ${ }^{* * *} \mathrm{p} \leq 0.001$ and ${ }^{* * * *} \mathrm{p} \leq 0.0001$.

\section{VclcKOxCMT1A display impaired electrophysiological parameters.}

We assessed motor electrophysiological characteristics on sciatic nerves in all four groups (Figure 35a). Vinculin conditional knockout mice were indifferent from the wildtype, whereas CMT1A mice displayed the CMT1A typical slower nerve conduction velocity, decreased evoked compound muscle action potentials and prolonged distal motor latencies (Figure 35b- 
a

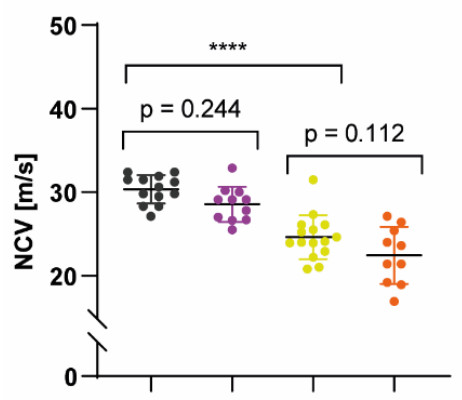

b

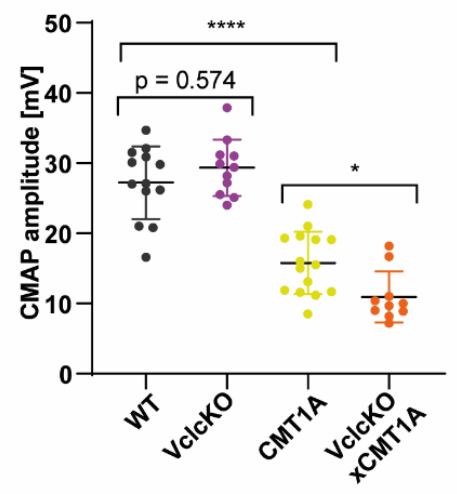

C

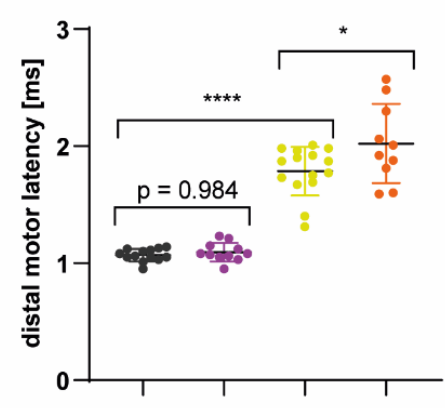

d

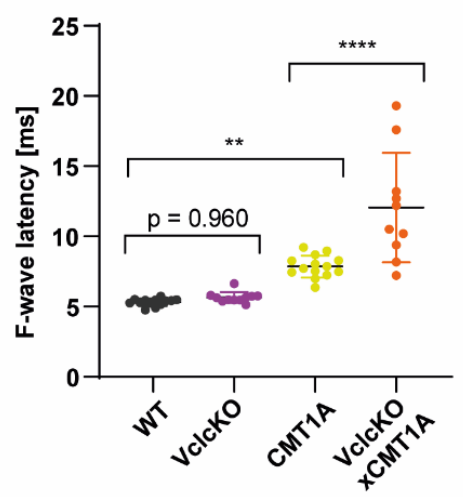

e

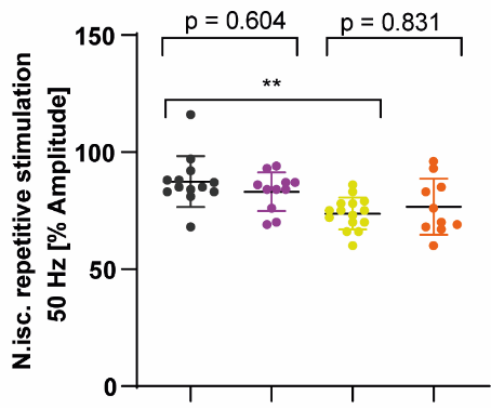

f

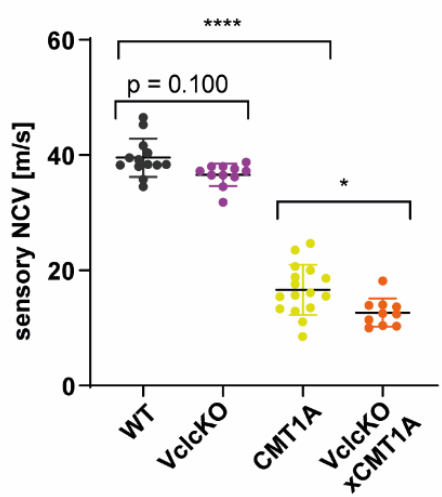

Figure 35: VclcKOxCMT1A double mutants show deteriorated motor electrophysiological characteristics.

(a) Motor nerve conduction velocities are unchanged in VclcKO mice (purple) and slower in CMT1A mice (yellow) compared to WT controls (grey) and show a trend to be more slowed in VclcKOxCMT1A double mutants (orange).

(b) The compound muscle action potential amplitude displays a decline in CMT1A mice (yellow) compared to WT controls (grey), which is significantly stronger when Vinculin lacks in CMT1A mice (orange).

(c) CMT1A mice (yellow) display a prolonged distal motor latency compared to WT controls (grey) that is more prolonged in CMT1A mice that lack Vinculin (orange) while Vinculin deficient mice (purple) are indifferent.

(d) F-wave latency is longer in CMT1A mice compared to WT controls. Thereby, VclcKOxCMT1A mice show a significantly prolonged latency compared to CMT1A mice.

(e) Repetitive stimulation of sciatic nerves displays decreased CMAP between the first and last burst in CMT1A mice, while VclcKOxCMT1A do not differ.

(f) Sensory nerve conduction velocities (sNCV) are unchanged in VclcKO mice (purple) and slower in CMT1A mice (yellow) compared to WT controls (grey). VclcKOxCMT1A double mutants (orange) display further reduced $\mathrm{sNCV}$.

Means are displayed \pm SD. WT $n=13, \operatorname{VclcKO} n=11$, CMT1A $n=15, \operatorname{VclcKOxCMT1A} n=10$ mice were analyzed at 18 weeks of age. Statistical analysis: One-way ANOVA with Sidak's multiple comparison test. ${ }^{*} \mathrm{p} \leq 0.05,{ }^{* *} \mathrm{p} \leq$ $0.01,{ }^{* *} \mathrm{p} \leq 0.001$ and ${ }^{* * * *} \mathrm{p} \leq 0.0001$. Electrophysiological measurements were performed by Theresa Kungl.

d). Moreover, CMT1A mice showed a longer f-wave latency and decreased amplitude after repetitive stimulation (Figure 35e-f).

Vinculin depletion and the resulting reduction in SLIs alone did not alter the behavioral and electrophysiological outcome, while a reduction of SLIs in the chronic disease state of CMT1A deteriorated the phenotype and electrophysiology. These results suggests a reduced number of SLI incisures does not affect the healthy, unstressed nerve while an increased SLI number is beneficial in chronic axon stress situations, as in CMT1A, whereas decreasing SLI number in the pathological situation impaired the phenotype, this hints towards a function for SLIs as routes for support from the Schwann cell to the axon. 


\section{Discussion}

Peripheral myelin protein of $22 \mathrm{kDa}$ (PMP22) is a protein of the compact myelin. Mutations regarding PMP22 are associated with different forms of peripheral neuropathies. PMP22 overexpression leads to Charcot-Marie-Tooth disease type 1A (CMT1A) and deficiency to hereditary neuropathy with liability to pressure palsies (HNPP). Treatment strategies are still rare due to incomplete understanding of the disease causing protein PMP22, its interaction partners and the molecular disease mechanisms.

In the first part, this study aimed at investigating the effect of PMP22 overexpression on the unfolded protein response and possible endoplasmic reticulum stress induction. Moreover, in the second part we tried to better understand dysregulations in growth signaling pathways and whether pharmacological targeting of these can provide therapeutical strategies in PMP22 gene-dosage related diseases. The last part investigates possible interaction partners of PMP22 and their importance in sustaining Schwann cell function during disease.

\subsection{The primary disease mechanism of CMT1A does not involve endoplasmic reticulum stress.}

The active myelination process requires a high amount of lipid and protein production in the Schwann cell, making it prone to endoplasmic reticulum stress (ER stress) and a subsequently activation of the unfolded protein response (UPR) (Lin and Popko 2009, D'Antonio et al 2009). In an animal model of Charcot-Marie-Tooth disease type 1B (CMT1B), which is caused by a mutation in the myelin protein zero (MPZ, P0), an upregulation of the UPR was observed and genetic deletion of the pro-apoptotic factors $C / E B P$ homologous protein $(\mathrm{CHOP})$ or growth arrest and DNA damage-inducible (GADD34) improved myelination (Wrabetz et al 2006, D'Antonio et al 2013). Moreover, pharmacological inhibition of GADD34 was shown to provide a therapeutical target in CMT1B (D'Antonio et al 2013, Das et al 2015). CMT1A is caused by an overexpression of PMP22 and similar to P0, PMP22 passes though the secretory pathway via ER and Golgi (Pareek et al 1993).

PMP22 is most strongly expressed around postnatal day 18 in the rat (Fledrich et al 2014). Therefore, we expected the highest probability for ER stress in CMT1A at this time point. In peripheral nerves of CMT1A rats, ER stress markers were unaltered at postnatal day 18, 9 weeks and 16 weeks of age, independent of the disease severity. Only in 47 weeks old CMT1A rats, an upregulation of the ER stress marker gadd34 was observed. Taken together, PMP22 overexpression in CMT1A does not cause ER stress during active myelination. Nevertheless, CMT1A rats at 47 weeks of age showed slight upregulation of an ER stress marker.

The overall ability of activating the UPR declines with aging, whereas an upregulation of gadd34 has been reported in aged rats (Paz Gavilan et al 2006, Naidoo 2009). We treated Schwann cell DRG co-cultures with a specific inhibitor of GADD34 and in agreement with the in vivo data and did not observe improved myelination in CMT1A. Moreover, mRNA 
expression analysis in the cultures did not reveal increased UPR gene expression. We have to keep in mind that the two weeks Schwann cell-DRG co-culture system we used might be not sufficient to display secondary effects of CMT1A pathology in vitro. Therefore, it can be of interest to further study the secondary mechanisms of ER stress in CMT1A, while the primary cause of the pathology is ER stress independent. The hypothesis of ER stress as a direct consequence of PMP22 overexpression has been discussed in the literature (D'Antonio et al 2009, Volpi et al 2016, Lin and Popko 2009) and the chosen animal or cell culture model seems to be critical.

Our results are confirmatory with studies in sural nerve biopsies of CMT1A patients which showed no PMP22 accumulation in the ER (Hanemann et al 2000). Contradictory, cultured dermal fibroblasts from CMT1A patients display a pronounced localization of overexpressed PMP22 in the ER and aggresome formation (Lee et al 2018a). PMP22 is expressed in various tissues throughout the body whereas PMP22 mRNA transcription is regulated by two promoters. One transcript is expressed in all cell types while the other transcript is specifically expressed in Schwann cells (Suter et al 1994; Bosse et al 1994). Hence, using dermal fibroblast might not serve as a sufficient model to directly translate the effects of PMP22 overexpression to the peripheral nervous system. Furthermore, aggresomes were only reported in fibroblasts of adult patients (Lee et al 2018a), in line with our results. Analysis of protein trafficking in strongly overexpressing homozygous CMT rats revealed that PMP22 is not retained in the ER and reached the Golgi compartment (Niemann et al 2000). In contrast, mouse models of CMT1A display aggresome formation in Schwann cells and an overload of the protein quality control system (Fortun et al 2006, Chittoor et al 2013). In these studies the C22 mouse model was used, which carries seven PMP22 copies (Huxley et al 1996). This mouse line is characterized by a severe histological as well as behavioral phenotype and the mice show a reduced life span. In contrast, the C61 CMT1A mouse and the CMT1A rat model more closely resemble the human phenotype, while carrying four and three PMP22 copies, respectively (Sereda et al 1996, Huxley et al 1998, Fledrich et al 2012b).

C61 CMT1A mice and CMT1A rats do not display aggresome formation, consistent with our findings of an unaltered ER stress marker expression in early development and adults. Accordingly, treatment with a UPR enhancing drug in vitro did not result in amelioration of myelination in a CMT1A SC-DRG co-culture system, in contrast to improved myelination in CMT1B (Das et al. 2015). Our results point to an involvement of the UPR in CMT1A only at old age, and it therefore seems unlikely that targeting the UPR provides effective treatment in CMT1A.

Taken together, we reject the idea that PMP22 overexpression causes ER stress and activation of the unfolded protein response during active myelination early in development. We further hypothesize that a gain of PMP22 function as a result of its overexpression in the Schwann cell is the key to the pathomechanism of CMT1A. Therefore, we studied molecular alterations related to PMP22 gene dosage to get better insight in the molecular role of PMP22 in CMT1A. 


\subsection{Does PMP22 control the timing of myelin growth?}

To get deeper insight in the molecular function of PMP22 and implications in disease we aimed at studying the effects of PMP22 dosage in animal models of Charcot-Marie-Tooth disease 1A (Huxley et al 1998, Sereda et al 1996) and hereditary neuropathy with liability to pressure palsies (Adlkofer et al 1995). PMP22 deficiency as a loss of function causes the formation of socalled tomacula, redundant myelin loops around the axon, while PMP22 overexpression and subsequently gain of function leads to an increase in hypo- and amyelinated large caliber axons. Already at postnatal day 18, the histological hallmarks are clearly visible in both animal models (Adlkofer et al 1997, Huxley et al 1998, Fledrich et al 2014). The reciprocal relationship suggests that PMP22 regulates myelin growth in the peripheral nervous system. One of the major myelin growth signaling pathways in the PNS is the PI3K/AKT/mTOR pathway, which was shown to be indeed PMP22 dosage-dependently regulated (Fledrich et al 2014). The $\mathrm{PI} 3 \mathrm{~K} / \mathrm{AKT} / \mathrm{mTOR}$ pathway is downregulated upon PMP22 overexpression and upregulated with PMP22 deficiency in animal models of CMT1A and HNPP, respectively. Thus, PMP22 negatively impacts the growth signaling pathway in the Schwann cell.

\section{PTEN is PMP22 gene-dosage dependently altered in CMT1A and HNPP.}

Phosphatase and tensin homolog (PTEN) antagonizes PI3K and inhibits the pathway by converting $\mathrm{PI}(3,4,5) \mathrm{P}_{3}$ to $\mathrm{PI}(4,5) 2$. Schwann cell specific deletion of PTEN results in an overactivation of the PI3K/AKT/mTOR pathway and a phenotype characterized by tomacula and outfoldings, similar to HNPP (Goebbels et al 2012). We found PTEN levels increased in CMT1A and decreased in HNPP. Therefore, we hypothesized that PMP22 regulates $\mathrm{PI} 3 \mathrm{~K} / \mathrm{AKT} / \mathrm{mTOR}$ dependent growth signaling upstream via PTEN. Then, counteracting the imbalanced PI3K/AKT/mTOR pathway in PMP22 related diseases by targeting either PTEN directly or processes downstream of PTEN can provide a therapeutical strategy.

\section{Inhibition of mTOR is beneficial in HNPP.}

We thus treated SC-DRG co-cultures of HNPP mice with the mTOR inhibitor Rapamycin and found a decrease in the number of myelin outfoldings. Translation of these findings in vivo displayed reduced tomacula formation accompanied with increased grip strength. Therefore, reducing mTOR activity is beneficial in HNPP. Hypermyelination and tomacula formation is also seen in other forms of CMT, such as CMT4B1 and CMT4B2, caused by mutations in myotubularin-related protein 2 (MTMR2) and 13 (MTMR13), respectively (Bolino et al 2000, Houlden et al 2001, Azzedine et al 2003). Recently, Ras-related protein Rab35 has been shown to be in complex with MTMRs and loss of function results in a similar focal hypermyelination phenotype in Schwann cell specific Rab35 knockout mice (Sawade et al 2020). The conversion of $\mathrm{PI}(4,5) \mathrm{P}_{2}$ to $\mathrm{PI}(3,4,5) \mathrm{P}_{3}$ is catalyzed by $\mathrm{PI} 3 \mathrm{~K}$ and subsequently the AKT/mTOR pathway is activated while PTEN reverses the reaction. Rapamycin treatment has shown to be beneficial in PTEN and Rab35 conditional knockout mice (Goebbels et al 2012, Sawade et al 2020). Thus, 
increased mTOR activity is a common observation in tomaculous peripheral neuropathies and Rapamycin administration provides a useful therapy strategy.

MTOR consists of two protein complexes, mTORC1 and mTORC2 that regulate different processes in the cell and Rapamycin only targets the mTORC1 complex (Laplante and Sabatini 2012). Treatment with Rapamycin and analogs (rapalogs) has already been tested and approved for clinical use in a variety of tumor diseases (Benjamin et al 2011). Nevertheless, often only limited clinical success was achieved because of negative feedback loops due to insufficient inhibition of mTOR ( $\mathrm{Li}$ et al 2014). A second generation of inhibitors competes with ATP in binding the kinase domain in mTOR and thereby, targeting mTORC1 and mTORC2 (Albert et al 2010). Moreover, a third generation of mTOR inhibitors, so-called Rapa-Links is recently investigated as they provide inhibition of both mTOR complexes with reduced side effects (Rodrik-Outmezguine et al 2016). Most clinical trials aimed at treating cancer, although mTOR inhibitors are also investigated in neurodegenerative diseases such as Parkinson's disease and Huntington's disease (Bove et al 2011). Thus, clinical translation of mTOR inhibition in tomaculous peripheral neuropathies is very promising.

\section{Increased PI3K/AKT/mTOR signaling by PTEN reduction improves myelination in CMT1A in vitro.}

In CMT1A, the PI3K/AKT/mTOR pathway is downregulated and PTEN protein levels increased. We used two different strategies to increase pathway activity via PTEN. In a first approach, SC-DRG co-cultures were treated with different dosages of VO-OHpic, a small vanadium complex specifically inhibiting PTEN (Rosivatz et al 2006). In wildtype cultures, the number of myelinated segments decreased with stronger inhibition of PTEN. Therefore, myelinating co-cultures are highly dosage-sensitive to PTEN inhibition. In CMT1A cultures, we observed increased segments with $50 \mathrm{nM}$ and $500 \mathrm{nM}$ inhibitor, while myelination was almost abolished with $5 \mu \mathrm{M}$ inhibitor. Increasing PI3K/AKT/mTOR signaling by inhibiting PTEN in CMT1A cultures improved myelination, although wildtype levels were never reached. We could not exclude an inhibition effect on axonal PTEN, therefore we made use of a second, genetic approach. We crossbred CMT1A with PTEN Schwann cell specific heterozygous knockout mice. Thereby, we could specifically decrease PTEN and increase $\mathrm{PI} 3 \mathrm{~K} / \mathrm{AKT} / \mathrm{mTOR}$ activity only in Schwann cells. Myelinating SC-DRG co-cultures of these animals revealed no effect of PTEN reduction alone on myelination, whereas PTEN reduced CMT1A cells showed increased myelinated segments. Axonal PTEN plays an important role in controlling axon outgrowth (Christie et al 2010) and inhibition of axonal and glial PTEN simultaneously decreased myelination in wildtype cultures while heterozygous PTEN deletion in Schwann cells alone had no effect on myelination in vitro. This demonstrates that glial PTEN dosage is crucial for proper myelination. Our in vitro results show that counteracting PTEN overexpression in CMT1A improves myelination in vitro, supporting the idea that PMP22 regulates myelin growth via PTEN. 
PTEN reduction increases myelin growth only in early development but does not overcome the differentiation defect.

We further analyzed the genetic PTEN reduction in CMT1A Schwann cells at postnatal day $18,12,16$ and 24 weeks of age in vivo. At postnatal day 18, PTEN heterozygous knockout in CMT1A Schwann cells decreased the number of amyelinated axons and increased myelin sheath thickness. Longitudinal myelination was not significantly altered but showed trends towards longer internodes with PTEN depletion. However, these early beneficial effects were not visible in adult animals, neither myelination nor behavior or electrophysiology were improved by PTEN deletion at 12 and 16 weeks of age, respectively. There were even tendencies towards deteriorated phenotype in 24 weeks old mice.

CMT1A is often described as a demyelinating neuropathy, whereas the term dysmyelination provides a more correct description the histological phenotype, as close examination reveals not only hypomyelination of preferable large axons but also hypermyelination, especially of small axons (Fledrich et al 2014). The earliest time point we examined was postnatal day 18. At this stage, increasing AKT activation by PTEN reduction improved myelin sheath thickness, however the axon diameter versus g-ratio distribution did not change. Thus, PTEN reduction and subsequently $\mathrm{AKT} / \mathrm{mTOR}$ activation improves the overall myelin growth but does not alter early differentiation defects in CMT1A.

\section{Does PMP22 play a dual function in mediating myelin growth?}

It was shown that PTEN deletion in Schwann cells leads to a delayed onset of myelination early postnatally (Figlia et al 2017) with full knockout in adults displaying tomacula formation whereas PTEN heterozygous knockout mice were unaltered (Goebbels et al 2012). Moreover, inducible deletion of PTEN in adult mice also activated myelin growth and subsequently tomacula formation (Figlia et al 2017). Similarly, the onset of myelination is delayed in PMP22 knockout mice, while tomacula formation occurs at later stages (Carenini et al 1999, Adlkofer et al 1995). These observations form a basis of a model in which PMP22 serves a dual function in timing myelination in the PNS. Early in development, PMP22 deficiency delays the onset of myelination and during later development it leads to hypermyelination and tomacula formation. In CMT1A, PMP22 overexpression causes hypermyelination of small caliber axons in early development and hypomyelination at later stages. Upon stronger PMP22 overexpression, as observed in homozygous CMT rats, Schwann cells and axons establish a 1:1 ratio but hardly any myelin is formed suggesting a dose dependency in the termination of myelination (Sereda et al 1996, Niemann et al 1999, Niemann et al 2000). These findings support the hypothesis of PMP22 in timing myelination. Early in development PMP22 is involved in the initiation of myelination, whereas its correct dosage is needed to terminate myelination (Figure 36).

Our results in vivo show which role the PI3K/AKT/mTOR pathway may play in this. Inhibition of mTOR from p21 on was beneficial in PMP22 deficient mice, while those mice still showed some tomacula. We conclude that the inhibition of mTOR is sufficient to provide the missing 

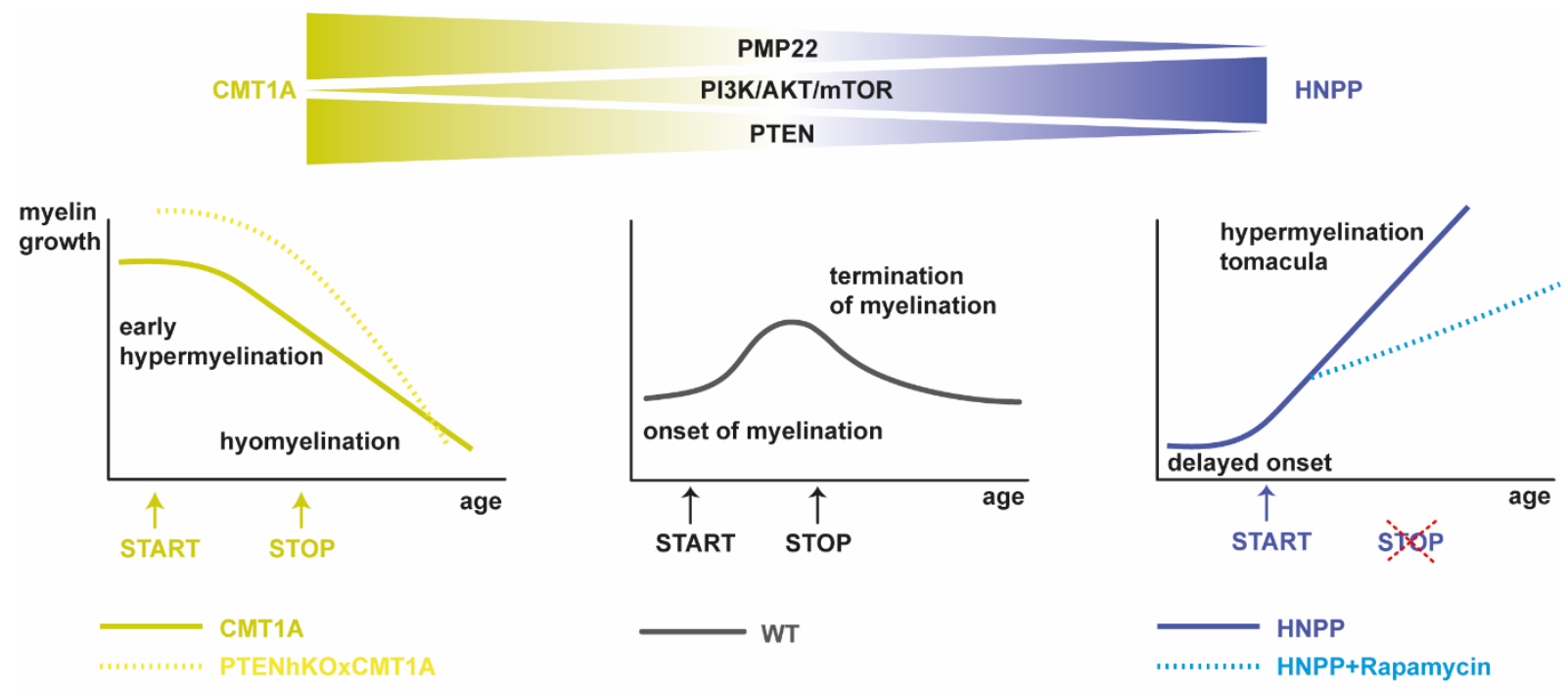

Figure 36: PMP22 in control of timing myelination?

PMP22 overexpression negatively correlates with the activation of PI3K/AKT/mTOR and positively correlates with the levels of the pathways major inhibitor PTEN. The panel on the lefts delineates myelin growth in CMT1A (yellow solid line) with early hypermyelination and hypomyelination at later stages. PTEN reduction (yellow dashed line) delays the termination of myelination. The middle panels depicts myelin growth in the wildtype (grey line). Myelin growth starts after radial sorting and terminates at late postnatal stages. The panel on the right delineates myelin growth in HNPP mice (blue solid line) that show a delayed onset of myelination and tomacula formation at later stages. Rapamycin treatment in HNPP mice is sufficient to induce the missing Stop signal and reduce tomacula formation.

termination signal in HNPP mice. We have not done an early developmental treatment and therefore, the impact of mTOR inhibition in the onset of myelination remains unclear. Supporting our findings, Figlia et al 2017 have proposed a dual role for mTOR in myelination, increased activation of mTOR suppresses myelination early in development to allow radial sorting and a later decline in pathway activity mediates proper myelin wrapping. Transferring this to the HNPP mice can explain the delayed onset due to increased mTOR early in development and myelin overgrowth at later stages by mTOR hyperactivity, which is improved through mTOR inhibition by Rapamycin.

In PMP22 overexpressing CMT1A mice, a genetic PTEN reduction increased myelination and decreased the number of amyelinated axons at p18, the earliest time point we evaluated. Thus, PTEN reduction allows thicker myelin sheath formation and myelination of more axons but importantly, this does not overcome the too early onset of myelination and subsequent differentiation phenotype in CMT1A. Fledrich et al 2014 described the altered differentiation phenotype as the primary disease cause in CMT1A caused by an imbalance between the $\mathrm{PI} 3 \mathrm{~K} / \mathrm{AKT} / \mathrm{mTOR}$ and the MEK/ERK pathway. Interestingly, increasing PI3K activity by treatment with exogenous or genetic increase of axonal Neuregulin 1 (Nrg1) type I rescued the signaling imbalance and improved the CMT1A phenotype in the long term (Fledrich et al 2014). Thereby, timing of the treatment was crucial. Early administration of exogenous Nrg1 type I before postnatal day 18 was beneficial, whereas treatment in adults was unsuccessful. Persistent glial induction of Nrg1 type I is observed after peripheral nerve injury and in demyelinating neuropathies such as CMT1A and CMT1B, whereas no difference is observed 
in HNPP mice (Stassart et al 2013, Fledrich et al 2019). Ablation of glial Nrg1 type I in CMT1A Schwann cells deteriorated the phenotype early in development at postnatal day 18 but ameliorated the phenotype in the long run by reducing hypermyelination of small caliber axons and decreasing onion bulb formation (Fledrich et al 2019). In agreement with our data, the rescued differentiation defect in Nrg1 type I depleted CMT1A Schwann cells did not alter AKT phosphorylation, indicating the differentiation defect is independent of PI3K/AKT/mTOR signaling. Moreover, glial Nrg1 type I overexpression induces hypermyelination of small axons without effecting PMP22 expression and thereby, suggesting PMP22 mediates the CMT1A pathomechanism upstream of NRG1 (Fledrich et al 2019).

Hence, the differentiation defect in CMT1A is mediated by PMP22 overexpression via Nrg1 type I independent of the PTEN-PI3K/AKT/mTOR pathway, whereas Nrg1 type I levels were unaltered in HNPP mice (Fledrich et al 2019). This can explain the positive effects of counteracting the PI3K/AKT/mTOR pathway in HNPP mice, whereas PTEN reduction slightly increased myelin growth early in development but did not overcome the differentiation defect in CMT1A. Further research is needed to untangle the complex interplay between PMP22 and the PTEN-PI3K/AKT/mTOR pathway in orchestrating the onset and termination of myelination in the Schwann cell. Investigation of interaction partners and localization of PMP22 can be of high importance in mediating the timing of myelination.

\subsection{Molecular role of PMP22.}

PMP22 dysregulation leads to the most common forms of CMT disease - CMT1A and HNPP. Therefore, understanding the molecular role of PMP22 in health and disease is of high interest to find potential therapeutic targets. Peripheral myelin protein of $22 \mathrm{kDa}$ was originally identified as growth arrest-specific 3 (gas3) in serum depleted fibroblasts (Schneider et al 1988). Moreover, gas3 was found to be highly expressed in Schwann cells and isolating the protein revealed a $22 \mathrm{kDa}$ glycosylated transmembrane protein - PMP22 (Spreyer et al 1991, Welcher et al 1991). Increased PMP22 amounts were shown to keep Schwann cells in a growth arrest state while PMP22 decrease promoted cell cycle progression (Zoidl et al 1995). Moreover, PMP22 was reported to indirectly interact with Integrins at the abaxonal site (Amici et al 2006, Poitelon et al 2018) and regulate f-actin dynamics and permeability of the peripheral nerve (Lee et al 2014, Guo et al 2014). Moreover PMP22 harbors cholesterol binding sites, important in lipid trafficking during myelination (Zhou et al 2019, Zhou et al 2020), while lipid genes are strongly downregulated and exogenous lipid supplementations is beneficial in CMT1A (Fledrich et al 2018). We have now shown a PMP22 dosage-dependent regulation of PTEN, a tumor suppressor gene and mediating growth arrest (Furnari et al 1998), similar to PMP22s first described function as a growth arrest gene. Our results suggest that PMP22 regulates $\mathrm{PI} 3 \mathrm{~K} / \mathrm{AKT} / \mathrm{mTOR}$ signaling dosage-dependently via PTEN, although the interaction mechanisms remain elusive. 


\section{Vinculin as a prospective interaction partner of PMP22 to stabilize PTEN.}

First immunoprecipitation and crosslinking studies did not show a direct protein-protein interaction of PMP22 and PTEN. Instead, these analysis revealed an enrichment in focal adhesion proteins cross-linked to PMP22 (Ewers, Arlt, unpublished data). A promising candidate was Vinculin, an actin-binding protein well described in focal adhesion assembly and regulation as well as cadherin-based cell-cell junctions (Bays and DeMali 2017, Ziegler et al 2006). Vinculin has been reported to control PTEN protein levels via a complex with $\beta$ Cadherin and Membrane-associated guanylate kinase inverted 2 (MAGI-2) (Subauste et al 2005). Consistently, we showed Vinculin protein level increased in CMT1A and decreased in HNPP. Therefore, we hypothesized that PMP22 acts on PTEN proteins via an interaction with Vinculin.

The molecular function of Vinculin in the Schwann cell has not been studied in detail, yet. Siems et al 2020 detected Vinculin in a peripheral myelin proteome and Beppu et al 2015 found autoantibodies against Vinculin in a form of chronic inflammatory demyelinating polyneuropathy, suggesting an important role for Vinculin in health and disease of the peripheral nerve. We demonstrated Vinculin localization to focal adhesions in primary Schwann cells as well as bands of Cajal and paranodal loops in peripheral nerve teased fiber preparations. Similarly, we showed PTEN localization to bands of Cajal, in which an interaction of the proteins could occur. The bands of Cajal are cytoplasmic channels at the outermost abaxonal site of the Schwann cell and other adhesion-related proteins such as focal adhesion kinase (FAK) and integrins are found at this site (Grove et al 2007, Feltri et al 2002). Both are involved in the formation of focal adhesion complexes and PMP22 was reported to directly interact with $\alpha 6 \beta 4$ integrin in early development (Amici et al 2006), although this interaction is not visible in older animals (Poitelon et al 2018). Integrins connect to the cytoskeleton through focal adhesion proteins vinculin and talin (Ziegler et al 2008) and PMP22 deficient nerves display altered cytoskeletal arrangements (Lee et al 2014). Similarly to PTEN (Tamura et al 1998), Vinculin controls shape and migration behavior of cells (Rodriguez Fernandez et al 1992), which is deteriorated in CMT1A primary Schwann cells as they are less polarized and migrate slower. Moreover, CMT1A teased fiber preparations display enlarged and disorganized bands of Cajal. We speculated that Vinculin as a potential interaction partner of PMP22 could explain the observed changes in actin cytoskeleton mediated functions as well as a subsequent stabilization of PTEN and impairments in growth signaling.

\section{Vinculin does not prevent PTEN from degradation in Schwann cells.}

We asked the question whether a Schwann cell specific deletion of Vinculin disturbs the potential interaction with PTEN and subsequently leads to a PTEN conditional knockout like phenotype. A full knockout of Vinculin is lethal at early embryonic stages ( $\mathrm{Xu}$ et al 1998). Deletion of Vinculin in cardiomyocytes reduced PTEN protein levels and activated the downstream P-AKT pathway (Zemljic-Harpf et al 2014). We generated $\mathrm{Vcl}^{\mathrm{fl} / \mathrm{fl}} \mathrm{Dhh}{ }^{\mathrm{cre} /+}$ mice (VclcKO) to specifically delete Vinculin in Schwann cells and further study the involvement 
in PTEN stabilization in vivo. Contrary to our expectations, VclcKO mice did not resemble the PTEN conditional knockout phenotype and showed normal myelination, behavior and electrophysiology. Moreover, protein analysis revealed unaltered PTEN protein levels in Vinculin depleted peripheral nerves and in HEK cells transiently overexpressing Vinculin. We concluded that Vinculin does not serve as a link between PTEN and PMP22 in Schwann cells.

Nevertheless, PMP22 gene-dosage dependently alters PTEN and Vinculin protein levels in animal models of CMT1A and HNPP. PTEN has already been well described in the Schwann cell (Goebbels et al 2012, Figlia et al 2017) and PTEN dysregulation results in a perturbed $\mathrm{PI} 3 \mathrm{~K} / \mathrm{AKT} / \mathrm{mTOR}$ signaling and myelin growth (Fledrich et al 2014). As primarily dysregulated Vinculin did not influence PTEN protein levels in the Schwann cell, the dysregulation of PTEN probably occurs independent of Vinculin. However, PMP22 strongly influences Vinculin dosage and we were eager to further investigate the role of Vinculin in the Schwann cell and CMT1A.

\subsection{A role for Schmidt-Lanterman incisures in sustaining Schwann cell function in Charcot-Marie Tooth disease 1A.}

\section{Vinculin conditional knockout mice provide a model to study Schmidt-Lanterman Incisure function in the peripheral nervous system.}

Animals with a Schwann cell specific deletion of Vinculin (VclcKO) displayed no alterations in myelin sheath thickness, behavior and electrophysiology. Vinculin is expressed in bands of Cajal, which are important in regulating longitudinal myelin growth (Court et al 2004, Sherman et al 2001). Closer examination of longitudinal myelination in VclcKO mice revealed unaltered internodal length and normal appearing bands of Cajal. Interestingly, the formation of Schmidt-Lanterman incisures (SLIs) lagged behind in early postnatal time points (p6) and SLI numbers were reduced by $\sim 25 \%$ in adult mice. Therefore Vinculin is not needed to form SLIs, but nevertheless seems to control their number. SLIs are cytoplasmic myelin channels in the compact myelin internode and their formation and molecular function is largely unknown. They are thought to be radial transport routes through the compact myelin to metabolically support the axon, provide mechanical flexibility to the peripheral nerve and be sites of membrane addition and degeneration in myelin maintenance (Hall and Williams 1970, Terada et al 2019). Different animal model were described to display increased numbers of SLIs due to alterations in myelin proteins (Gould et al 1995; Sherman et al 2012, our data), deficiency in myelin lipid production (Hoshi et al 2007) or developmental signaling molecules (SharghiNamini et al 2006), and the number of SLIs also increases after peripheral nerve injury (Berger and Gupta 2006). SLI are thought to couple axon-glia functions in the PNS and provide 'shortcut routes' for metabolic nutrients from the Schwann cell to the axon. An increase in the number of SLIs could therefore be indicative of axons in need of metabolic support. A loss of Schmidt-Lanterman incisures has been reported for Periaxin null mice (Gillespie et al 2000, Sherman et al 2012) and myelin protein zero (MPZ, P0) deficient mice (Yin et al 2008), although 


\section{DISCUSSION}

in contrast to the VclcKO mice, those mice display severe histological alterations with overall unstable, partly uncompacted myelin. The absence of such general deficits in Vinculin conditional knockout mice thus provides for the first time a model to study the molecular function of SLIs in health and disease of the peripheral nervous system.

\section{Schmidt-Lanterman Incisures are needed to sustain Schwann cell function in CMT1A.}

We crossbred CMT1A mice, which display increased SLI numbers, with Vinculin conditional knockout mice to shed further light on the function of SLIs in CMT1A. Vinculin depletion in CMT1A Schwann cells reduced SLI number in the same manner as previously observed in VclcKO mice ( $25 \%)$. This reduction is already seen early in development and persists throughout development. Vinculin depleted CMT1A Schwann cells do increase SLI formation compared to the wildtype, although not to the extend observed in CMT1A mice. Importantly, this reduction of SLIs in CMT1A leads to a strong deterioration of the electrophysiological and behavioral phenotype. Moreover, the number of amyelinated axons is increased in Vinculin depleted CMT1A mice, while myelinated axons display normal myelin sheath thickness. Behavioral data revealed an especially strong decrease in hind limb grip strength of Vinculin depleted CMT1A mice, indicating a more distally pronounced phenotype. These results support the hypothesis of disturbed transport along the internodes and SLIs. Further research on distal versus proximal pathology in Vinculin depleted CMT1A nerves could provide a better understanding of the phenotype. These observation lead to the conclusion that increased
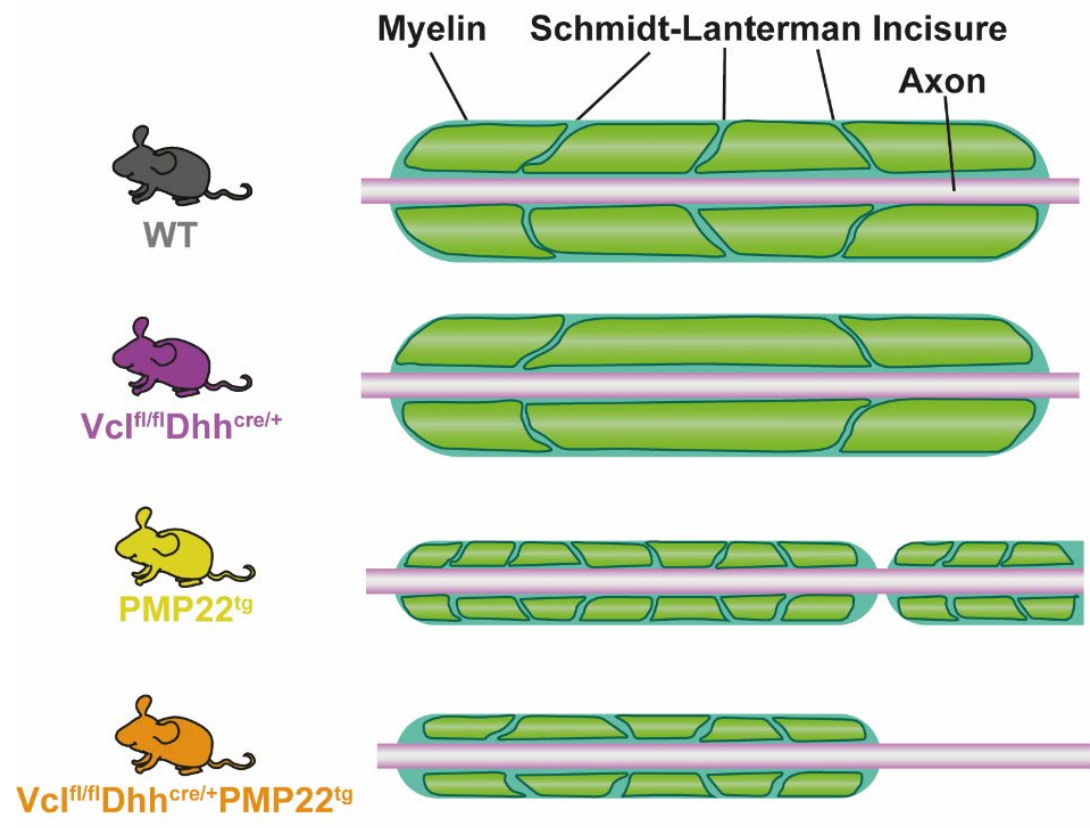

Mean \#SLI/
internode [\%]

$100 \%$

$75 \%$ normal

$150 \%$ impaired $125 \%$

phenotype normal

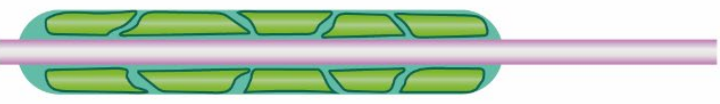

\section{strongly} impaired

Figure 37: A role of Schmidt-Lanterman Incisures for sustaining Schwann cell function in CMT1A.

Scheme displaying normal SLI number in wildtype mice (WT) and reduced SLI number in VclcKO mice (purple) without effecting the phenotype. CMT1A mice (yellow) show increased numbers of SLI, thinner myelin sheath with an impaired phenotype. Reduction of SLI by Vinculin depletion in CMT1A Schwann cells (orange) results in decreased numbers of SLI and increased numbers of amyelinated axons, indicating increased SLI number is beneficial in CMT1A. 
numbers of Schmidt-Lanterman incisures are indeed beneficial in neuropathy, as they are needed to sustain Schwann cell function in CMT1A (Figure 37).

We can only speculate on the specific mechanisms of Vinculin in regulating the formation and number of SLIs. Vinculin itself is not expressed in SLIs but in the adjacent bands of Cajal, which link SLIs longitudinally. Bands of Cajal and SLIs are actin-rich structures and Vinculin as a facting binding protein could be important in mediating the cytoskeletal dynamics during SLI formation (Huang et al 2017, Johnson and Craig 1995). Moreover, Vinculin is part of the cadherin-catenin adhesion junctional complex (Hazan et al 1997, Thomas et al 2013), and interesting data has been published on the role of E-cadherin in SLIs. Conditional deletion of E-Cadherin in Schwann cells did not obviously affect SLI formation, although unfortunately no quantification of SLI number was performed in these mice (Young et al 2002). However, adenoviral delivery of a dominant acting E-cadherin mutant protein in sciatic nerves results in a loss of SLI and adherens junctions leading to increased cytoplasm in the compact myelin of transduced nerves (Tricaud et al 2005). Interestingly, the authors of this study claim Ecadherin is needed for maintaining SLIs in myelinating Schwann cells by recruitment of p120 catenin.

Future research on Vinculin mediated signaling pathways and alterations in associated proteins will shed light on the process of SLI formation and its role in disease. We have not investigated SLI and Cajal band ultrastructure, yet. First ultrastructural analysis of SLIs in CMT1A rats have shown accumulation of organelles and disorganization of SLI architecture (Robert Fledrich, Wiebke Möbius, unpublished data), indicating SLIs as 'transport routes' not only from the axon to the Schwann cell but in maintaining functions in the Schwann cell itself. Moreover, SLI number is increased after peripheral nerve injury (Berger and Gupta 2006, Ghabriel and Allt 1980), which led us expect that performing nerve crush experiments in Vinculin depleted nerves will provide a better understanding of SLI function in remyelination. 


\subsection{Conclusions}

In this study, different aspects of the molecular role of peripheral myelin protein of $22 \mathrm{kDa}$ (PMP22) in the Schwann cell in health and disease were investigated.

In the first part we showed that PMP22 overexpression in Charcot-Marie-Tooth disease type 1A (CMT1A) does not cause an upregulation of the unfolded protein response during active myelination. Therefore, we conclude that the pathomechanisms of PMP22 gene-dosage related diseases result from a gain (CMT1A) or partial loss (HNPP) of function.

Furthermore, we studied alterations in animal models of PMP22 gene-dosage to gain insight into the molecular function of PMP22. Previous studies demonstrated a negative regulation of the PI3K/AKT/mTOR growth signaling pathway by PMP22, while the interactions remained unclear. We showed that protein levels of PTEN, the major inhibitor of the pathway, are correlating with PMP22 protein levels thereby suggesting that PMP22 mediates alterations of the PI3K/AKT/mTOR pathway via PTEN.

We then explored the therapeutic potential of influencing the PI3K/AKT/mTOR pathway in PMP22 gene-dosage related diseases either by targeting PTEN directly or downstream components.

We showed that inhibition of mTOR by Rapamycin is beneficial in treating PMP22 deficient HNPP mice. Since nowadays second and third generation inhibitors with less side effects and stronger inhibition of mTOR exist, it would be of high interest to study the effects of improved inhibition not only during late postnatal stages but early in development.

Moreover, we demonstrated that either pharmacologic inhibition or genetic reduction of PTEN improves myelination in CMT1A Schwann cell-DRG co-cultures in vitro. In contrast to the genetic reduction, pharmacological inhibition of PTEN showed strong effects also on myelination in wildtype cultures, indicating negative effects of axonal PTEN inhibition. Therefore, we did not apply the PTEN inhibitor but further explored the genetic Schwann cell specific PTEN reduction in CMT1A in vivo. PTEN reduction increased the number and thickness of myelinated axons at postnatal day 18, whereas older animals were unaltered. Further, PTEN reduction and subsequent activation of the PI3K/AKT/mTOR signaling pathway was not sufficient to overcome the differentiation defect due to upregulation of glial NRG1 type I and subsequent hyperactivation of the MEK/ERK signaling pathway. Our data show that this differentiation defect is PTEN-PI3K/AKT/mTOR independent. Further research is needed to investigate the link between PMP22 and NRG1 type I and the overall role of NRG1 mediated pathways in the pathomechanism of CMT1A, in order to then evaluate whether those provide therapeutical strategies in targeting CMT1A and overcome the differentiation defect. We concluded that PMP22 serves different function in mediating the timing of myelination. Analysis of both PMP22 interaction partners and subcellular localization is highly important to further elucidate the molecular role of PMP22. 
Further, we investigated the role of a potential PMP22 interaction partner in the Schwann cell. Vinculin is an actin-binding protein enriched in crosslinking studies of PMP22 and provided a promising interaction partner of PMP22 for several reasons. First, Vinculin was reported to stabilize PTEN and Vinculin depletion in other cell types increases PI3K/AKT/mTOR activation. Indeed, Vinculin protein levels were PMP22 gene-dosage dependently altered in animal models of CMT1A and HNPP. Second, cellular mechanisms regulated by Vinculin expression such as cell shape and migration were changed in PMP22 overexpressing primary Schwann cells. Third, Vinculin is expressed in the abaxonal cytoplasmic bands of Cajal in the Schwann cell, which are strongly disorganized in CMT1A peripheral nerves. Contrary to our expectations, Schwann cell specific depletion of Vinculin did not resemble the PTEN conditional knockout phenotype in vivo. Thereby, neither PTEN protein levels nor myelination, behavior or electrophysiology was impaired upon Vinculin depletion in Schwann cells. We rejected the hypothesis of PMP22 stabilizing PTEN via Vinculin interaction in the Schwann cell. It remains open how PMP22 influences the PTEN-PI3K/AKT/mTOR pathway. Further exploration of possible protein-protein interaction partners related to signaling pathways can provide further mechanistic understanding in the complex interplay. To this end, we will have to overcome the specific challenges of interaction studies on PMP22 as a small hydrophobic transmembrane protein has an increased risk of false positive findings.

Analysis of Vinculin conditional knockout mice (VclcKO) revealed an interesting observation. While the overall phenotype and myelination were unchanged, peripheral axons of VclcKO mice displayed a reduced number in Schmidt-Lanterman Incisures (SLI). SLIs are believed to provide shortcut routes for metabolites from the Schwann to the axon and might be sites of membrane addition and degeneration. However, experimental evidence is incomplete. To our knowledge, VclcKO mice provide the first model to study the molecular role of SLI. CMT1A and other peripheral neuropathies show increased numbers of SLI and crossbreeding VclcKO mice with CMT1A mice decreased SLI number and deteriorated the overall phenotype. We concluded that increased SLI are needed to sustain Schwann cell function in CMT1A. We initiated the analysis of peripheral nerves from VclcKO ex vivo to shed light on the role of metabolite restriction and transport via SLI. Moreover, SLI are increased after peripheral nerve injury and nerve compression experiments in VclcKO mice may clarify the role of SLIs in injury and remyelination.

Taken together, this study sheds light on the molecular role of PMP22 in the regulation of Schwann cell myelination and provides knowledge on therapeutical targets for PMP22 genedosage related diseases. Moreover, a novel mouse model for studying Schmidt-Lanterman Incisures is introduced and offers first evidence for SLI in maintaining Schwann cell function in peripheral neuropathies. 


\section{References}

Adlkofer K, Frei R, Neuberg DH, Zielasek J, Toyka KV, Suter U. 1997. Heterozygous peripheral myelin protein 22-deficient mice are affected by a progressive demyelinating tomaculous neuropathy. J Neurosci 17: 4662-71

Adlkofer K, Martini R, Aguzzi A, Zielasek J, Toyka KV, Suter U. 1995. Hypermyelination and demyelinating peripheral neuropathy in Pmp22-deficient mice. Nat Genet 11: 274-80

Albert S, Serova M, Dreyer C, Sablin MP, Faivre S, Raymond E. 2010. New inhibitors of the mammalian target of rapamycin signaling pathway for cancer. Expert Opin Investig Drugs 19: 919-30

Amici SA, Dunn WA, Jr., Murphy AJ, Adams NC, Gale NW, Valenzuela DM, Yancopoulos GD, Notterpek L. 2006. Peripheral myelin protein 22 is in complex with alpha6beta4 integrin, and its absence alters the Schwann cell basal lamina. J Neurosci 26: 1179-89

Archelos JJ, Roggenbuck K, Schneider-Schaulies J, Toyka KV, Hartung HP. 1993. Detection and quantification of antibodies to the extracellular domain of P0 during experimental allergic neuritis. J Neurol Sci 117: 197-205

Armati PJ, Mathey EK. 2013. An update on Schwann cell biology--immunomodulation, neural regulation and other surprises. J Neurol Sci 333: 68-72

Armati PJ, Pollard JD, Gatenby P. 1990. Rat and human Schwann cells in vitro can synthesize and express MHC molecules. Muscle Nerve 13: 106-16

Azzedine H, Bolino A, Taieb T, Birouk N, Di Duca M, Bouhouche A, Benamou S, Mrabet A, Hammadouche T, Chkili T, Gouider R, Ravazzolo R, Brice A, Laporte J, LeGuern E. 2003. Mutations in MTMR13, a new pseudophosphatase homologue of MTMR2 and Sbf1, in two families with an autosomal recessive demyelinating form of CharcotMarie-Tooth disease associated with early-onset glaucoma. Am J Hum Genet 72: 114153

Bai Y, Zhang X, Katona I, Saporta MA, Shy ME, O'Malley HA, Isom LL, Suter U, Li J. 2010. Conduction block in PMP22 deficiency. J Neurosci 30: 600-8

Bays JL, DeMali KA. 2017. Vinculin in cell-cell and cell-matrix adhesions. Cell Mol Life Sci 74: 2999-3009

Benjamin D, Colombi M, Moroni C, Hall MN. 2011. Rapamycin passes the torch: a new generation of mTOR inhibitors. Nat Rev Drug Discov 10: 868-80

Benninger Y, Thurnherr T, Pereira JA, Krause S, Wu X, Chrostek-Grashoff A, Herzog D, Nave KA, Franklin RJ, Meijer D, Brakebusch C, Suter U, Relvas JB. 2007. Essential and distinct roles for cdc42 and rac1 in the regulation of Schwann cell biology during peripheral nervous system development. J Cell Biol 177: 1051-61

Beppu M, Sawai S, Satoh M, Mori M, Kazami T, Misawa S, Shibuya K, Ishibashi M, Sogawa K, Kado S, Kodera Y, Nomura F, Kuwabara S. 2015. Autoantibodies against vinculin in patients with chronic inflammatory demyelinating polyneuropathy. J Neuroimmunol 287: 9-15

Berger BL, Gupta R. 2006. Demyelination secondary to chronic nerve compression injury alters Schmidt-Lanterman incisures. J Anat 209: 111-8

Birchmeier C, Nave KA. 2008. Neuregulin-1, a key axonal signal that drives Schwann cell growth and differentiation. Glia 56: 1491-7

Bird TD. 1993. Charcot-Marie-Tooth (CMT) Hereditary Neuropathy Overview In GeneReviews((R)), ed. MP Adam, HH Ardinger, RA Pagon, SE Wallace, LJH Bean, et al. Seattle (WA) 
Boerkoel CF, Inoue K, Reiter LT, Warner LE, Lupski JR. 1999. Molecular Mechanisms for CMT1A Duplication and HNPP Deletion. Ann N Y Acad Sci 883: 22-35

Bolino A, Bolis A, Previtali SC, Dina G, Bussini S, Dati G, Amadio S, Del Carro U, Mruk DD, Feltri ML, Cheng CY, Quattrini A, Wrabetz L. 2004. Disruption of Mtmr2 produces CMT4B1-like neuropathy with myelin outfolding and impaired spermatogenesis. J Cell Biol 167: 711-21

Bolino A, Muglia M, Conforti FL, LeGuern E, Salih MA, Georgiou DM, Christodoulou K, Hausmanowa-Petrusewicz I, Mandich P, Schenone A, Gambardella A, Bono F, Quattrone A, Devoto M, Monaco AP. 2000. Charcot-Marie-Tooth type 4B is caused by mutations in the gene encoding myotubularin-related protein-2. Nat Genet 25: 17-9

Bosse F, Zoidl G, Wilms S, Gillen CP, Kuhn HG, Muller HW. 1994. Differential expression of two mRNA species indicates a dual function of peripheral myelin protein PMP22 in cell growth and myelination. J Neurosci Res 37: 529-37

Bove J, Martinez-Vicente M, Vila M. 2011. Fighting neurodegeneration with rapamycin: mechanistic insights. Nat Rev Neurosci 12: 437-52

Brancolini C, Edomi P, Marzinotto S, Schneider C. 2000. Exposure at the cell surface is required for gas3/PMP22 To regulate both cell death and cell spreading: implication for the Charcot-Marie-Tooth type 1A and Dejerine-Sottas diseases. Mol Biol Cell 11: 2901-14

Brancolini C, Marzinotto S, Edomi P, Agostoni E, Fiorentini C, Muller HW, Schneider C. 1999. Rho-dependent regulation of cell spreading by the tetraspan membrane protein Gas3/PMP22. Mol Biol Cell 10: 2441-59

Brinkmann BG, Agarwal A, Sereda MW, Garratt AN, Muller T, Wende H, Stassart RM, Nawaz S, Humml C, Velanac V, Radyushkin K, Goebbels S, Fischer TM, Franklin RJ, Lai C, Ehrenreich H, Birchmeier C, Schwab MH, Nave KA. 2008. Neuregulin-1/ErbB signaling serves distinct functions in myelination of the peripheral and central nervous system. Neuron 59: 581-95

Burridge K, Mangeat P. 1984. An interaction between vinculin and talin. Nature 308: 744-6

Butovsky O, Weiner HL. 2018. Microglial signatures and their role in health and disease. Nat Rev Neurosci 19: 622-35

Carenini S, Neuberg D, Schachner M, Suter U, Martini R. 1999. Localization and functional roles of PMP22 in peripheral nerves of P0-deficient mice. Glia 28: 256-64

Chandrasekar I, Stradal TE, Holt MR, Entschladen F, Jockusch BM, Ziegler WH. 2005. Vinculin acts as a sensor in lipid regulation of adhesion-site turnover. J Cell Sci 118: 1461-72

Chittoor VG, Sooyeon L, Rangaraju S, Nicks JR, Schmidt JT, Madorsky I, Narvaez DC, Notterpek L. 2013. Biochemical characterization of protein quality control mechanisms during disease progression in the C22 mouse model of CMT1A. ASN Neuro 5: e00128

Christie KJ, Webber CA, Martinez JA, Singh B, Zochodne DW. 2010. PTEN inhibition to facilitate intrinsic regenerative outgrowth of adult peripheral axons. J Neurosci 30: 930615

Colby J, Nicholson R, Dickson KM, Orfali W, Naef R, Suter U, Snipes GJ. 2000. PMP22 carrying the trembler or trembler-J mutation is intracellularly retained in myelinating Schwann cells. Neurobiol Dis 7: 561-73

Connor JH, Weiser DC, Li S, Hallenbeck JM, Shenolikar S. 2001. Growth arrest and DNA damage-inducible protein GADD34 assembles a novel signaling complex containing protein phosphatase 1 and inhibitor 1. Mol Cell Biol 21: 6841-50 
Cotter L, Ozcelik M, Jacob C, Pereira JA, Locher V, Baumann R, Relvas JB, Suter U, Tricaud N. 2010. Dlg1-PTEN interaction regulates myelin thickness to prevent damaging peripheral nerve overmyelination. Science 328: 1415-8

Court FA, Hewitt JE, Davies K, Patton BL, Uncini A, Wrabetz L, Feltri ML. 2009. A laminin-2, dystroglycan, utrophin axis is required for compartmentalization and elongation of myelin segments. J Neurosci 29: 3908-19

Court FA, Sherman DL, Pratt T, Garry EM, Ribchester RR, Cottrell DF, Fleetwood-Walker SM, Brophy PJ. 2004. Restricted growth of Schwann cells lacking Cajal bands slows conduction in myelinated nerves. Nature 431: 191-5

Court FA, Wrabetz L, Feltri ML. 2006. Basal lamina: Schwann cells wrap to the rhythm of space-time. Curr Opin Neurobiol 16: 501-7

D'Antonio M, Feltri ML, Wrabetz L. 2009. Myelin under stress. J Neurosci Res 87: 3241-9

D'Antonio M, Musner N, Scapin C, Ungaro D, Del Carro U, Ron D, Feltri ML, Wrabetz L. 2013. Resetting translational homeostasis restores myelination in Charcot-Marie-Tooth disease type 1B mice. J Exp Med 210: 821-38

D'Urso D, Ehrhardt P, Muller HW. 1999. Peripheral myelin protein 22 and protein zero: a novel association in peripheral nervous system myelin. J Neurosci 19: 3396-403

D'Urso D, Prior R, Greiner-Petter R, Gabreels-Festen AA, Muller HW. 1998. Overloaded endoplasmic reticulum-Golgi compartments, a possible pathomechanism of peripheral neuropathies caused by mutations of the peripheral myelin protein PMP22. J Neurosci 18: $731-40$

Das I, Krzyzosiak A, Schneider K, Wrabetz L, D'Antonio M, Barry N, Sigurdardottir A, Bertolotti A. 2015. Preventing proteostasis diseases by selective inhibition of a phosphatase regulatory subunit. Science 348: 239-42

Domenech-Estevez E, Baloui H, Meng X, Zhang Y, Deinhardt K, Dupree JL, Einheber S, Chrast R, Salzer JL. 2016. Akt Regulates Axon Wrapping and Myelin Sheath Thickness in the PNS. J Neurosci 36: 4506-21

DuBridge RB, Tang P, Hsia HC, Leong PM, Miller JH, Calos MP. 1987. Analysis of mutation in human cells by using an Epstein-Barr virus shuttle system. Mol Cell Biol 7: 379-87

Dyck PJ, Lambert EH. 1968. Lower motor and primary sensory neuron diseases with peroneal muscular atrophy. I. Neurologic, genetic, and electrophysiologic findings in hereditary polyneuropathies. Arch Neurol 18: 603-18

Fabbretti E, Edomi P, Brancolini C, Schneider C. 1995. Apoptotic phenotype induced by overexpression of wild-type gas3/PMP22: its relation to the demyelinating peripheral neuropathy CMT1A. Genes Dev 9: 1846-56

Feltri ML, Graus Porta D, Previtali SC, Nodari A, Migliavacca B, Cassetti A, Littlewood-Evans A, Reichardt LF, Messing A, Quattrini A, Mueller U, Wrabetz L. 2002. Conditional disruption of beta 1 integrin in Schwann cells impedes interactions with axons. J Cell Biol 156: 199-209

Feltri ML, Poitelon Y, Previtali SC. 2016. How Schwann Cells Sort Axons: New Concepts. Neuroscientist 22: 252-65

Fernando RN, Cotter L, Perrin-Tricaud C, Berthelot J, Bartolami S, Pereira JA, Gonzalez S, Suter U, Tricaud N. 2016. Optimal myelin elongation relies on YAP activation by axonal growth and inhibition by Crb3/Hippo pathway. Nat Commun 7: 12186

Figlia G, Gerber D, Suter U. 2018. Myelination and mTOR. Glia 66: 693-707

Figlia G, Norrmen C, Pereira JA, Gerber D, Suter U. 2017. Dual function of the PI3K-AktmTORC1 axis in myelination of the peripheral nervous system. Elife 6 
Fledrich R, Abdelaal T, Rasch L, Bansal V, Schutza V, Brugger B, Luchtenborg C, Prukop T, Stenzel J, Rahman RU, Hermes D, Ewers D, Mobius W, Ruhwedel T, Katona I, Weis J, Klein D, Martini R, Bruck W, Muller WC, Bonn S, Bechmann I, Nave KA, Stassart RM, Sereda MW. 2018. Targeting myelin lipid metabolism as a potential therapeutic strategy in a model of CMT1A neuropathy. Nat Commun 9: 3025

Fledrich R, Akkermann D, Schutza V, Abdelaal TA, Hermes D, Schaffner E, Soto-Bernardini MC, Gotze T, Klink A, Kusch K, Krueger M, Kungl T, Frydrychowicz C, Mobius W, Bruck W, Mueller WC, Bechmann I, Sereda MW, Schwab MH, Nave KA, Stassart RM. 2019. NRG1 type I dependent autoparacrine stimulation of Schwann cells in onion bulbs of peripheral neuropathies. Nat Commun 10: 1467

Fledrich R, Schlotter-Weigel B, Schnizer TJ, Wichert SP, Stassart RM, Meyer zu Horste G, Klink A, Weiss BG, Haag U, Walter MC, Rautenstrauss B, Paulus W, Rossner MJ, Sereda MW. 2012a. A rat model of Charcot-Marie-Tooth disease 1A recapitulates disease variability and supplies biomarkers of axonal loss in patients. Brain 135: 72-87

Fledrich R, Stassart RM, Klink A, Rasch LM, Prukop T, Haag L, Czesnik D, Kungl T, Abdelaal TA, Keric N, Stadelmann C, Bruck W, Nave KA, Sereda MW. 2014. Soluble neuregulin1 modulates disease pathogenesis in rodent models of Charcot-Marie-Tooth disease 1A. Nat Med 20: 1055-61

Fledrich R, Stassart RM, Sereda MW. 2012b. Murine therapeutic models for Charcot-MarieTooth (CMT) disease. Br Med Bull 102: 89-113

Fontanini A, Chies R, Snapp EL, Ferrarini M, Fabrizi GM, Brancolini C. 2005. Glycanindependent role of calnexin in the intracellular retention of Charcot-Marie-tooth $1 \mathrm{~A}$ Gas3/PMP22 mutants. J Biol Chem 280: 2378-87

Fortun J, Go JC, Li J, Amici SA, Dunn WA, Jr., Notterpek L. 2006. Alterations in degradative pathways and protein aggregation in a neuropathy model based on PMP22 overexpression. Neurobiol Dis 22: 153-64

Funfschilling U, Supplie LM, Mahad D, Boretius S, Saab AS, Edgar J, Brinkmann BG, Kassmann CM, Tzvetanova ID, Mobius W, Diaz F, Meijer D, Suter U, Hamprecht B, Sereda MW, Moraes CT, Frahm J, Goebbels S, Nave KA. 2012. Glycolytic oligodendrocytes maintain myelin and long-term axonal integrity. Nature 485: 517-21

Furnari FB, Huang HJ, Cavenee WK. 1998. The phosphoinositol phosphatase activity of PTEN mediates a serum-sensitive G1 growth arrest in glioma cells. Cancer Res 58: 5002-8

Gabreels-Festen AA, Bolhuis PA, Hoogendijk JE, Valentijn LJ, Eshuis EJ, Gabreels FJ. 1995. Charcot-Marie-Tooth disease type 1A: morphological phenotype of the 17p duplication versus PMP22 point mutations. Acta Neuropathol 90: 645-9

Garcia CA, Malamut RE, England JD, Parry GS, Liu P, Lupski JR. 1995. Clinical variability in two pairs of identical twins with the Charcot-Marie-Tooth disease type 1A duplication. Neurology 45: 2090-3

Ghabriel MN, Allt G. 1980. The role of Schmidt-Lantermann incisures in remyelination. Folia Morphol (Praha) 28: 129-33

Giaume C, Koulakoff A, Roux L, Holcman D, Rouach N. 2010. Astroglial networks: a step further in neuroglial and gliovascular interactions. Nat Rev Neurosci 11: 87-99

Gillespie CS, Sherman DL, Fleetwood-Walker SM, Cottrell DF, Tait S, Garry EM, Wallace VC, Ure J, Griffiths IR, Smith A, Brophy PJ. 2000. Peripheral demyelination and neuropathic pain behavior in periaxin-deficient mice. Neuron 26: 523-31 
Goebbels S, Oltrogge JH, Wolfer S, Wieser GL, Nientiedt T, Pieper A, Ruhwedel T, Groszer M, Sereda MW, Nave KA. 2012. Genetic disruption of Pten in a novel mouse model of tomaculous neuropathy. EMBO Mol Med 4: 486-99

Goldmann WH. 2016. Role of vinculin in cellular mechanotransduction. Cell Biol Int 40: 241-56

Gould RM, Byrd AL, Barbarese E. 1995. The number of Schmidt-Lanterman incisures is more than doubled in shiverer PNS myelin sheaths. J Neurocytol 24: 85-98

Groszer M, Erickson R, Scripture-Adams DD, Lesche R, Trumpp A, Zack JA, Kornblum HI, Liu $X, W u H .2001$. Negative regulation of neural stem/progenitor cell proliferation by the Pten tumor suppressor gene in vivo. Science 294: 2186-9

Grove M, Komiyama NH, Nave K-A, Grant SG, Sherman DL, Brophy PJ. 2007. FAK is required for axonal sorting by Schwann cells. Journal of Cell Biology 176: 277-82

Guo J, Wang L, Zhang Y, Wu J, Arpag S, Hu B, Imhof BA, Tian X, Carter BD, Suter U, Li J. 2014. Abnormal junctions and permeability of myelin in PMP22-deficient nerves. Ann Neurol 75: 255-65

Hall SM, Williams PL. 1970. Studies on the "incisures" of Schmidt and Lanterman. J Cell Sci 6: 767-91

Hanemann CO, D'Urso D, Gabreels-Festen AA, Muller HW. 2000. Mutation-dependent alteration in cellular distribution of peripheral myelin protein 22 in nerve biopsies from Charcot-Marie-Tooth type 1A. Brain 123 ( Pt 5): 1001-6

Harding HP, Novoa I, Zhang Y, Zeng H, Wek R, Schapira M, Ron D. 2000. Regulated translation initiation controls stress-induced gene expression in mammalian cells. Mol Cell 6: 1099-108

Harding HP, Zhang Y, Ron D. 1999. Protein translation and folding are coupled by an endoplasmic-reticulum-resident kinase. Nature 397: 271-4

Hartline DK, Colman DR. 2007. Rapid conduction and the evolution of giant axons and myelinated fibers. Curr Biol 17: R29-35

Hasse B, Bosse F, Hanenberg H, Muller HW. 2004. Peripheral myelin protein $22 \mathrm{kDa}$ and protein zero: domain specific trans-interactions. Mol Cell Neurosci 27: 370-8

Hazan RB, Kang L, Roe S, Borgen PI, Rimm DL. 1997. Vinculin is associated with the Ecadherin adhesion complex. J Biol Chem 272: 32448-53

Heller BA, Ghidinelli M, Voelkl J, Einheber S, Smith R, Grund E, Morahan G, Chandler D, Kalaydjieva L, Giancotti F, King RH, Fejes-Toth AN, Fejes-Toth G, Feltri ML, Lang F, Salzer JL. 2014. Functionally distinct PI 3-kinase pathways regulate myelination in the peripheral nervous system. Journal of Cell Biology 204: 1219-36

Hildebrand C, Bowe CM, Remahl IN. 1994. Myelination and myelin sheath remodelling in normal and pathological PNS nerve fibres. Prog Neurobiol 43: 85-141

Hoshi T, Suzuki A, Hayashi S, Tohyama K, Hayashi A, Yamaguchi Y, Takeuchi K, Baba H. 2007. Nodal protrusions, increased Schmidt-Lanterman incisures, and paranodal disorganization are characteristic features of sulfatide-deficient peripheral nerves. Glia 55: 584-94

Houlden H, King RH, Wood NW, Thomas PK, Reilly MM. 2001. Mutations in the 5' region of the myotubularin-related protein 2 (MTMR2) gene in autosomal recessive hereditary neuropathy with focally folded myelin. Brain 124: 907-15

Hu B, Arpag S, Zhang X, Mobius W, Werner H, Sosinsky G, Ellisman M, Zhang Y, Hamilton A, Chernoff J, Li J. 2016. Tuning PAK Activity to Rescue Abnormal Myelin Permeability in HNPP. PLoS Genet 12: e1006290 
Huang DL, Bax NA, Buckley CD, Weis WI, Dunn AR. 2017. Vinculin forms a directionally asymmetric catch bond with F-actin. Science 357: 703-06

Huh SH, Do HJ, Lim HY, Kim DK, Choi SJ, Song H, Kim NH, Park JK, Chang WK, Chung HM, Kim JH. 2007. Optimization of $25 \mathrm{kDa}$ linear polyethylenimine for efficient gene delivery. Biologicals 35: 165-71

Huxley C, Passage E, Manson A, Putzu G, Figarella-Branger D, Pellissier JF, Fontes M. 1996. Construction of a mouse model of Charcot-Marie-Tooth disease type 1A by pronuclear injection of human YAC DNA. Hum Mol Genet 5: 563-9

Huxley C, Passage E, Robertson AM, Youl B, Huston S, Manson A, Saberan-Djoniedi D, Figarella-Branger D, Pellissier JF, Thomas PK, Fontes M. 1998. Correlation between varying levels of PMP22 expression and the degree of demyelination and reduction in nerve conduction velocity in transgenic mice. Hum Mol Genet 7: 449-58

Jaegle M, Ghazvini M, Mandemakers W, Piirsoo M, Driegen S, Levavasseur F, Raghoenath S, Grosveld F, Meijer D. 2003. The POU proteins Brn-2 and Oct-6 share important functions in Schwann cell development. Genes Dev 17: 1380-91

Jessen KR, Mirsky R. 2005. The origin and development of glial cells in peripheral nerves. Nat Rev Neurosci 6: 671-82

Jessen KR, Mirsky R. 2016. The repair Schwann cell and its function in regenerating nerves. J Physiol 594: 3521-31

Johnson RP, Craig SW. 1995. F-actin binding site masked by the intramolecular association of vinculin head and tail domains. Nature 373: 261-4

Kumar N, Muley S, Pakiam A, Parry GJ. 2002. Phenotypic Variability Leads to Underrecognition of HNPP. J Clin Neuromuscul Dis 3: 106-12

Laplante M, Sabatini DM. 2012. mTOR signaling in growth control and disease. Cell 149: 27493

Larocca JN, Norton WT. 2006. Isolation of Myelin. In Current Protocols in Cell Biology: John Wiley \& Sons, Inc.

Lee JO, Yang H, Georgescu MM, Di Cristofano A, Maehama T, Shi Y, Dixon JE, Pandolfi P, Pavletich NP. 1999. Crystal structure of the PTEN tumor suppressor: implications for its phosphoinositide phosphatase activity and membrane association. Cell 99: 323-34

Lee S, Amici S, Tavori H, Zeng WM, Freeland S, Fazio S, Notterpek L. 2014. PMP22 is critical for actin-mediated cellular functions and for establishing lipid rafts. J Neurosci 34: 16140-52

Lee S, Bazick H, Chittoor-Vinod V, Al Salihi MO, Xia G, Notterpek L. 2018a. Elevated Peripheral Myelin Protein 22, Reduced Mitotic Potential, and Proteasome Impairment in Dermal Fibroblasts from Charcot-Marie-Tooth Disease Type 1A Patients. Am J Pathol 188: 728-38

Lee YR, Chen M, Pandolfi PP. 2018b. The functions and regulation of the PTEN tumour suppressor: new modes and prospects. Nat Rev Mol Cell Biol 19: 547-62

Lewis RA, Sumner AJ, Shy ME. 2000. Electrophysiological features of inherited demyelinating neuropathies: A reappraisal in the era of molecular diagnosis. Muscle Nerve 23: 1472-87

Li J, Kim SG, Blenis J. 2014. Rapamycin: one drug, many effects. Cell Metab 19: 373-9

Li J, Krajewski K, Shy ME, Lewis RA. 2002. Hereditary neuropathy with liability to pressure palsy: the electrophysiology fits the name. Neurology 58: 1769-73

Li J, Parker B, Martyn C, Natarajan C, Guo J. 2013. The PMP22 gene and its related diseases. Mol Neurobiol 47: 673-98 
Liaw D, Marsh DJ, Li J, Dahia PL, Wang SI, Zheng Z, Bose S, Call KM, Tsou HC, Peacocke M, Eng C, Parsons R. 1997. Germline mutations of the PTEN gene in Cowden disease, an inherited breast and thyroid cancer syndrome. Nat Genet 16: 64-7

Lin W, Popko B. 2009. Endoplasmic reticulum stress in disorders of myelinating cells. Nature Neuroscience 12: 379-85

Lloyd AF, Miron VE. 2019. The pro-remyelination properties of microglia in the central nervous system. Nat Rev Neurol 15: 447-58

Lobsiger CS, Taylor V, Suter U. 2002. The early life of a Schwann cell. Biol Chem 383: 245-53

Lupski JR. 1992. An inherited DNA rearrangement and gene dosage effect are responsible for the most common autosomal dominant peripheral neuropathy: Charcot-Marie-Tooth disease type 1A. Clin Res 40: 645-52

Macklin WB. 2010. The myelin brake: when enough is enough. Sci Signal 3: pe32

Mandich P, James R, Nassani S, Defferrari R, Bellone E, Mancardi G, Schenone A, Abbruzzese M, Rocchi M, Ajmar F, et al. 1995. Molecular diagnosis of hereditary neuropathy with liability to pressure palsies (HNPP) by detection of 17p11.2 deletion in Italian patients. J Neurol 242: 295-8

Manfioletti G, Ruaro ME, Del Sal G, Philipson L, Schneider C. 1990. A growth arrest-specific (gas) gene codes for a membrane protein. Mol Cell Biol 10: 2924-30

Martini R, Mohajeri MH, Kasper S, Giese KP, Schachner M. 1995. Mice doubly deficient in the genes for $\mathrm{P0}$ and myelin basic protein show that both proteins contribute to the formation of the major dense line in peripheral nerve myelin. J Neurosci 15: 4488-95

Martyn CN, Hughes RA. 1997. Epidemiology of peripheral neuropathy. J Neurol Neurosurg Psychiatry 62: 310-8

Maxwell MH. 1978. Two rapid and simple methods used for the removal of resins from 1.0 micron thick epoxy sections. J Microsc 112: 253-5

Michailov GV, Sereda MW, Brinkmann BG, Fischer TM, Haug B, Birchmeier C, Role L, Lai C, Schwab MH, Nave KA. 2004. Axonal neuregulin-1 regulates myelin sheath thickness. Science 304: 700-3

Mitsios JV, Prevost N, Kasirer-Friede A, Gutierrez E, Groisman A, Abrams CS, Wang Y, Litvinov RI, Zemljic-Harpf A, Ross RS, Shattil SJ. 2010. What is vinculin needed for in platelets? J Thromb Haemost 8: 2294-304

Monk KR, Oshima K, Jors S, Heller S, Talbot WS. 2011. Gpr126 is essential for peripheral nerve development and myelination in mammals. Development 138: 2673-80

Mouton P, Tardieu S, Gouider R, Birouk N, Maisonobe T, Dubourg O, Brice A, LeGuern E, Bouche P. 1999. Spectrum of clinical and electrophysiologic features in HNPP patients with the 17p11.2 deletion. Neurology 52: 1440-6

Naef R, Adlkofer K, Lescher B, Suter U. 1997. Aberrant protein trafficking in Trembler suggests a disease mechanism for hereditary human peripheral neuropathies. Mol Cell Neurosci 9: $13-25$

Naidoo N. 2009. The endoplasmic reticulum stress response and aging. Rev Neurosci 20: 23-37

Nave KA. 2010. Myelination and the trophic support of long axons. Nat Rev Neurosci 11: 27583

Nave KA, Werner HB. 2014. Myelination of the nervous system: mechanisms and functions. Annu Rev Cell Dev Biol 30: 503-33

Newbern J, Birchmeier C. 2010. Nrg1/ErbB signaling networks in Schwann cell development and myelination. Semin Cell Dev Biol 21: 922-8 
Niemann S, Sereda MW, Rossner M, Stewart H, Suter U, Meinck HM, Griffiths IR, Nave KA. 1999. The "CMT rat": peripheral neuropathy and dysmyelination caused by transgenic overexpression of PMP22. Ann N Y Acad Sci 883: 254-61

Niemann S, Sereda MW, Suter U, Griffiths IR, Nave KA. 2000. Uncoupling of myelin assembly and schwann cell differentiation by transgenic overexpression of peripheral myelin protein 22. J Neurosci 20: 4120-8

Nobbio L, Vigo T, Abbruzzese M, Levi G, Brancolini C, Mantero S, Grandis M, Benedetti L, Mancardi G, Schenone A. 2004. Impairment of PMP22 transgenic Schwann cells differentiation in culture: implications for Charcot-Marie-Tooth type $1 \mathrm{~A}$ disease. Neurobiol Dis 16: 263-73

Nodari A, Zambroni D, Quattrini A, Court FA, D'Urso A, Recchia A, Tybulewicz VL, Wrabetz L, Feltri ML. 2007. Beta1 integrin activates Rac1 in Schwann cells to generate radial lamellae during axonal sorting and myelination. J Cell Biol 177: 1063-75

Norrmen C, Figlia G, Lebrun-Julien F, Pereira JA, Trotzmuller M, Kofeler HC, Rantanen V, Wessig C, van Deijk AL, Smit AB, Verheijen MH, Ruegg MA, Hall MN, Suter U. 2014. mTORC1 controls PNS myelination along the mTORC1-RXRgamma-SREBP-lipid biosynthesis axis in Schwann cells. Cell Rep 9: 646-60

Noseda R, Belin S, Piguet F, Vaccari I, Scarlino S, Brambilla P, Martinelli Boneschi F, Feltri ML, Wrabetz L, Quattrini A, Feinstein E, Huganir RL, Bolino A. 2013. DDIT4/REDD1/RTP801 is a novel negative regulator of Schwann cell myelination. J Neurosci 33: 15295-305

Notterpek L, Roux KJ, Amici SA, Yazdanpour A, Rahner C, Fletcher BS. 2001. Peripheral myelin protein 22 is a constituent of intercellular junctions in epithelia. Proc Natl Acad Sci U S A 98: 14404-9

Notterpek L, Ryan MC, Tobler AR, Shooter EM. 1999. PMP22 accumulation in aggresomes: implications for CMT1A pathology. Neurobiol Dis 6: 450-60

Notterpek L, Shooter EM, Snipes GJ. 1997. Upregulation of the endosomal-lysosomal pathway in the trembler-J neuropathy. J Neurosci 17: 4190-200

Novoa I, Zeng H, Harding HP, Ron D. 2001. Feedback inhibition of the unfolded protein response by GADD34-mediated dephosphorylation of eIF2alpha. J Cell Biol 153: 101122

Okamoto Y, Pehlivan D, Wiszniewski W, Beck CR, Snipes GJ, Lupski JR, Khajavi M. 2013. Curcumin facilitates a transitory cellular stress response in Trembler-J mice. Hum Mol Genet 22: 4698-705

Pareek S, Notterpek L, Snipes GJ, Naef R, Sossin W, Laliberte J, Iacampo S, Suter U, Shooter EM, Murphy RA. 1997. Neurons promote the translocation of peripheral myelin protein 22 into myelin. J Neurosci 17: 7754-62

Pareek S, Suter U, Snipes GJ, Welcher AA, Shooter EM, Murphy RA. 1993. Detection and processing of peripheral myelin protein PMP22 in cultured Schwann cells. J Biol Chem 268: $10372-9$

Pareyson D, Marchesi C. 2009. Diagnosis, natural history, and management of Charcot-MarieTooth disease. Lancet Neurol 8: 654-67

Paz Gavilan M, Vela J, Castano A, Ramos B, del Rio JC, Vitorica J, Ruano D. 2006. Cellular environment facilitates protein accumulation in aged rat hippocampus. Neurobiol Aging 27: 973-82

Pereira JA, Lebrun-Julien F, Suter U. 2012. Molecular mechanisms regulating myelination in the peripheral nervous system. Trends Neurosci 35: 123-34 
Poitelon Y, Lopez-Anido C, Catignas K, Berti C, Palmisano M, Williamson C, Ameroso D, Abiko K, Hwang Y, Gregorieff A, Wrana JL, Asmani M, Zhao R, Sim FJ, Wrabetz L, Svaren J, Feltri ML. 2016. YAP and TAZ control peripheral myelination and the expression of laminin receptors in Schwann cells. Nat Neurosci 19: 879-87

Poitelon Y, Matafora V, Silvestri N, Zambroni D, McGarry C, Serghany N, Rush T, Vizzuso D, Court FA, Bachi A, Wrabetz L, Feltri ML. 2018. A dual role for Integrin alpha6beta4 in modulating hereditary neuropathy with liability to pressure palsies. J Neurochem 145: 245-57

Poliak S, Peles E. 2003. The local differentiation of myelinated axons at nodes of Ranvier. Nat Rev Neurosci 4: 968-80

Rangaraju S, Verrier JD, Madorsky I, Nicks J, Dunn WA, Jr., Notterpek L. 2010. Rapamycin activates autophagy and improves myelination in explant cultures from neuropathic mice. J Neurosci 30: 11388-97

Rasband MN, Peles E. 2021. Mechanisms of node of Ranvier assembly. Nat Rev Neurosci 22: 720

Rodriguez Fernandez JL, Geiger B, Salomon D, Ben-Ze'ev A. 1992. Overexpression of vinculin suppresses cell motility in BALB/c 3 T3 cells. Cell Motil Cytoskeleton 22: 127-34

Rodrik-Outmezguine VS, Okaniwa M, Yao Z, Novotny CJ, McWhirter C, Banaji A, Won H, Wong W, Berger M, de Stanchina E, Barratt DG, Cosulich S, Klinowska T, Rosen N, Shokat KM. 2016. Overcoming mTOR resistance mutations with a new-generation mTOR inhibitor. Nature 534: 272-6

Ron D, Walter P. 2007. Signal integration in the endoplasmic reticulum unfolded protein response. Nat Rev Mol Cell Biol 8: 519-29

Rosivatz E, Matthews JG, McDonald NQ, Mulet X, Ho KK, Lossi N, Schmid AC, Mirabelli M, Pomeranz KM, Erneux C, Lam EW, Vilar R, Woscholski R. 2006. A small molecule inhibitor for phosphatase and tensin homologue deleted on chromosome 10 (PTEN). ACS Chem Biol 1: 780-90

Rosso G, Liashkovich I, Gess B, Young P, Kun A, Shahin V. 2014. Unravelling crucial biomechanical resilience of myelinated peripheral nerve fibres provided by the Schwann cell basal lamina and PMP22. Sci Rep 4: 7286

Roux KJ, Amici SA, Fletcher BS, Notterpek L. 2005. Modulation of Epithelial Morphology, Monolayer Permeability, and Cell Migration by Growth Arrest Specific 3/Peripheral Myelin Protein 22. Molecular Biology of the Cell 16: 1142-51

Rushton WA. 1951. A theory of the effects of fibre size in medullated nerve. J Physiol 115: 10122

Russo M, Laura M, Polke JM, Davis MB, Blake J, Brandner S, Hughes RA, Houlden H, Bennett DL, Lunn MP, Reilly MM. 2011. Variable phenotypes are associated with PMP22 missense mutations. Neuromuscul Disord 21: 106-14

Ryan MC, Shooter EM, Notterpek L. 2002. Aggresome formation in neuropathy models based on peripheral myelin protein 22 mutations. Neurobiol Dis 10: 109-18

Salzer JL. 2015. Schwann cell myelination. Cold Spring Harb Perspect Biol 7: a020529

Sancho S, Young P, Suter U. 2001. Regulation of Schwann cell proliferation and apoptosis in PMP22-deficient mice and mouse models of Charcot-Marie-Tooth disease type 1A. Brain 124: 2177-87

Saporta MA, Katona I, Lewis RA, Masse S, Shy ME, Li J. 2009. Shortened internodal length of dermal myelinated nerve fibres in Charcot-Marie-Tooth disease type 1A. Brain 132: 3263-73 
Sawade L, Grandi F, Mignanelli M, Patino-Lopez G, Klinkert K, Langa-Vives F, Di Guardo R, Echard A, Bolino A, Haucke V. 2020. Rab35-regulated lipid turnover by myotubularins represses mTORC1 activity and controls myelin growth. Nat Commun 11: 2835

Scheuner D, Song B, McEwen E, Liu C, Laybutt R, Gillespie P, Saunders T, Bonner-Weir S, Kaufman RJ. 2001. Translational control is required for the unfolded protein response and in vivo glucose homeostasis. Mol Cell 7: 1165-76

Schneider C, King RM, Philipson L. 1988. Genes specifically expressed at growth arrest of mammalian cells. Cell 54: 787-93

Schultz RL, Karlsson U. 1965. Fixation of the Central Nervous System for Electron Microscopy by Aldehyde Perfusion. Ii. Effect of Osmolarity, Ph of Perfusate, and Fixative Concentration. J Ultrastruct Res 12: 187-206

Sereda M, Griffiths I, Puhlhofer A, Stewart H, Rossner MJ, Zimmerman F, Magyar JP, Schneider A, Hund E, Meinck HM, Suter U, Nave KA. 1996. A transgenic rat model of Charcot-Marie-Tooth disease. Neuron 16: 1049-60

Sereda MW, Nave KA. 2006. Animal models of Charcot-Marie-Tooth disease type 1A. Neuromolecular Med 8: 205-16

Shapiro L, Doyle JP, Hensley P, Colman DR, Hendrickson WA. 1996. Crystal structure of the extracellular domain from $\mathrm{P} 0$, the major structural protein of peripheral nerve myelin. Neuron 17: 435-49

Sharghi-Namini S, Turmaine M, Meier C, Sahni V, Umehara F, Jessen KR, Mirsky R. 2006. The structural and functional integrity of peripheral nerves depends on the glial-derived signal desert hedgehog. J Neurosci 26: 6364-76

Sheean ME, McShane E, Cheret C, Walcher J, Muller T, Wulf-Goldenberg A, Hoelper S, Garratt AN, Kruger M, Rajewsky K, Meijer D, Birchmeier W, Lewin GR, Selbach M, Birchmeier C. 2014. Activation of MAPK overrides the termination of myelin growth and replaces Nrg1/ErbB3 signals during Schwann cell development and myelination. Genes Dev 28: 290-303

Sherman DL, Brophy PJ. 2005. Mechanisms of axon ensheathment and myelin growth. Nat Rev Neurosci 6: 683-90

Sherman DL, Fabrizi C, Gillespie CS, Brophy PJ. 2001. Specific disruption of a schwann cell dystrophin-related protein complex in a demyelinating neuropathy. Neuron 30: 677-87

Sherman DL, Wu LM, Grove M, Gillespie CS, Brophy PJ. 2012. Drp2 and periaxin form Cajal bands with dystroglycan but have distinct roles in Schwann cell growth. J Neurosci 32: 9419-28

Siems SB, Jahn O, Eichel MA, Kannaiyan N, Wu LMN, Sherman DL, Kusch K, Hesse D, Jung RB, Fledrich R, Sereda MW, Rossner MJ, Brophy PJ, Werner HB. 2020. Proteome profile of peripheral myelin in healthy mice and in a neuropathy model. Elife 9

Snipes GJ, Suter U, Welcher AA, Shooter EM. 1992. Characterization of a novel peripheral nervous system myelin protein (PMP-22/SR13). J Cell Biol 117: 225-38

Song MS, Salmena L, Pandolfi PP. 2012. The functions and regulation of the PTEN tumour suppressor. Nat Rev Mol Cell Biol 13: 283-96

Spreyer P, Kuhn G, Hanemann CO, Gillen C, Schaal H, Kuhn R, Lemke G, Muller HW. 1991. Axon-regulated expression of a Schwann cell transcript that is homologous to a 'growth arrest-specific' gene. EMBO J 10: 3661-8

Stassart RM, Fledrich R, Velanac V, Brinkmann BG, Schwab MH, Meijer D, Sereda MW, Nave KA. 2013. A role for Schwann cell-derived neuregulin-1 in remyelination. Nat Neurosci 16: $48-54$ 
Steck PA, Pershouse MA, Jasser SA, Yung WK, Lin H, Ligon AH, Langford LA, Baumgard ML, Hattier T, Davis T, Frye C, Hu R, Swedlund B, Teng DH, Tavtigian SV. 1997. Identification of a candidate tumour suppressor gene, MMAC1, at chromosome 10q23.3 that is mutated in multiple advanced cancers. Nat Genet 15: 356-62

Subauste MC, Nalbant P, Adamson ED, Hahn KM. 2005. Vinculin controls PTEN protein level by maintaining the interaction of the adherens junction protein beta-catenin with the scaffolding protein MAGI-2. J Biol Chem 280: 5676-81

Suter U, Moskow JJ, Welcher AA, Snipes GJ, Kosaras B, Sidman RL, Buchberg AM, Shooter EM. 1992a. A leucine-to-proline mutation in the putative first transmembrane domain of the 22-kDa peripheral myelin protein in the trembler-J mouse. Proc Natl Acad Sci U $S$ A 89: 4382-6

Suter U, Snipes GJ, Schoener-Scott R, Welcher AA, Pareek S, Lupski JR, Murphy RA, Shooter EM, Patel PI. 1994. Regulation of tissue-specific expression of alternative peripheral myelin protein-22 (PMP22) gene transcripts by two promoters. J Biol Chem 269: 25795808

Suter U, Welcher AA, Ozcelik T, Snipes GJ, Kosaras B, Francke U, Billings-Gagliardi S, Sidman RL, Shooter EM. 1992b. Trembler mouse carries a point mutation in a myelin gene. Nature 356: 241-4

Tamura M, Gu J, Matsumoto K, Aota S, Parsons R, Yamada KM. 1998. Inhibition of cell migration, spreading, and focal adhesions by tumor suppressor PTEN. Science 280: 1614-7

Taveggia C, Zanazzi G, Petrylak A, Yano H, Rosenbluth J, Einheber S, Xu X, Esper RM, Loeb JA, Shrager P, Chao MV, Falls DL, Role L, Salzer JL. 2005. Neuregulin-1 type III determines the ensheathment fate of axons. Neuron 47: 681-94

Terada N, Saitoh Y, Kamijo A, Yamauchi J, Ohno N, Sakamoto T. 2019. Structures and Molecular Composition of Schmidt-Lanterman Incisures. Adv Exp Med Biol 1190: 18198

Thomas PK, Marques W, Jr., Davis MB, Sweeney MG, King RH, Bradley JL, Muddle JR, Tyson J, Malcolm S, Harding AE. 1997. The phenotypic manifestations of chromosome 17p11.2 duplication. Brain 120 ( Pt 3): 465-78

Thomas WA, Boscher C, Chu YS, Cuvelier D, Martinez-Rico C, Seddiki R, Heysch J, Ladoux B, Thiery JP, Mege RM, Dufour S. 2013. alpha-Catenin and vinculin cooperate to promote high E-cadherin-based adhesion strength. J Biol Chem 288: 4957-69

Timmerman V, Raeymaekers P, De Jonghe P, De Winter G, Swerts L, Jacobs K, Gheuens J, Martin JJ, Vandenberghe A, Van Broeckhoven C. 1990. Assignment of the CharcotMarie-Tooth neuropathy type 1 (CMT 1a) gene to 17p11.2-p12. Am J Hum Genet 47: 6805

Tobler AR, Notterpek L, Naef R, Taylor V, Suter U, Shooter EM. 1999. Transport of Trembler$\mathrm{J}$ mutant peripheral myelin protein 22 is blocked in the intermediate compartment and affects the transport of the wild-type protein by direct interaction. J Neurosci 19: 202736

Tricaud N. 2017. Myelinating Schwann Cell Polarity and Mechanically-Driven Myelin Sheath Elongation. Front Cell Neurosci 11: 414

Tricaud N, Perrin-Tricaud C, Bruses JL, Rutishauser U. 2005. Adherens junctions in myelinating Schwann cells stabilize Schmidt-Lanterman incisures via recruitment of p120 catenin to E-cadherin. J Neurosci 25: 3259-69

van Dort J. 2018. Aberration correction in STED 
microscopy. Georg-August University School of Science (GAUSS), Physics of Biological and Complex Systems

Verhamme C, King RH, ten Asbroek AL, Muddle JR, Nourallah M, Wolterman R, Baas F, van Schaik IN. 2011. Myelin and axon pathology in a long-term study of PMP22overexpressing mice. J Neuropathol Exp Neurol 70: 386-98

Volpi VG, Touvier T, D'Antonio M. 2016. Endoplasmic Reticulum Protein Quality Control Failure in Myelin Disorders. Front Mol Neurosci 9: 162

Volterra A, Meldolesi J. 2005. Astrocytes, from brain glue to communication elements: the revolution continues. Nat Rev Neurosci 6: 626-40

Walter P, Ron D. 2011. The unfolded protein response: from stress pathway to homeostatic regulation. Science 334: 1081-6

Welcher AA, Suter U, De Leon M, Snipes GJ, Shooter EM. 1991. A myelin protein is encoded by the homologue of a growth arrest-specific gene. Proc Natl Acad Sci U S A 88: 7195-9

Winkler U, Hirrlinger PG, Sestu M, Wilhelm F, Besser S, Zemljic-Harpf AE, Ross RS, Bornschein G, Krugel U, Ziegler WH, Hirrlinger J. 2013. Deletion of the cell adhesion adaptor protein vinculin disturbs the localization of GFAP in Bergmann glial cells. Glia 61: 1067-83

Wrabetz L, D'Antonio M, Pennuto M, Dati G, Tinelli E, Fratta P, Previtali S, Imperiale D, Zielasek J, Toyka K, Avila RL, Kirschner DA, Messing A, Feltri ML, Quattrini A. 2006. Different intracellular pathomechanisms produce diverse Myelin Protein Zero neuropathies in transgenic mice. J Neurosci 26: 2358-68

Xu W, Baribault H, Adamson ED. 1998. Vinculin knockout results in heart and brain defects during embryonic development. Development 125: 327-37

Yin X, Kidd GJ, Nave KA, Trapp BD. 2008. P0 protein is required for and can induce formation of schmidt-lantermann incisures in myelin internodes. J Neurosci 28: 7068-73

Young P, Boussadia O, Berger P, Leone D, Charnay P, Kemler R, Suter U. 2002. E-cadherin Is Required for the Correct Formation of Autotypic Adherens Junctions of the Outer Mesaxon but Not for the Integrity of Myelinated Fibers of Peripheral Nerves. Molecular and Cellular Neuroscience 21: 341-51

Yu WM, Feltri ML, Wrabetz L, Strickland S, Chen ZL. 2005. Schwann cell-specific ablation of laminin gamma1 causes apoptosis and prevents proliferation. J Neurosci 25: 4463-72

Zemljic-Harpf AE, Godoy JC, Platoshyn O, Asfaw EK, Busija AR, Domenighetti AA, Ross RS. 2014. Vinculin directly binds zonula occludens-1 and is essential for stabilizing connexin-43-containing gap junctions in cardiac myocytes. Journal of Cell Science 127: 1104-16

Zemljic-Harpf AE, Miller JC, Henderson SA, Wright AT, Manso AM, Elsherif L, Dalton ND, Thor AK, Perkins GA, McCulloch AD, Ross RS. 2007. Cardiac-myocyte-specific excision of the vinculin gene disrupts cellular junctions, causing sudden death or dilated cardiomyopathy. Mol Cell Biol 27: 7522-37

Zhou Y, Borchelt D, Bauson JC, Fazio S, Miles JR, Tavori H, Notterpek L. 2020. Subcellular diversion of cholesterol by gain- and loss-of-function mutations in PMP22. Glia 68: 2300-15

Zhou Y, Miles JR, Tavori H, Lin M, Khoshbouei H, Borchelt DR, Bazick H, Landreth GE, Lee S, Fazio S, Notterpek L. 2019. PMP22 Regulates Cholesterol Trafficking and ABCA1Mediated Cholesterol Efflux. J Neurosci 39: 5404-18

Ziegler WH, Gingras AR, Critchley DR, Emsley J. 2008. Integrin connections to the cytoskeleton through talin and vinculin. Biochem Soc Trans 36: 235-9 


\section{REFERENCES}

Ziegler WH, Liddington RC, Critchley DR. 2006. The structure and regulation of vinculin. Trends Cell Biol 16: 453-60

Zoidl G, Blass-Kampmann S, D'Urso D, Schmalenbach C, Muller HW. 1995. Retroviralmediated gene transfer of the peripheral myelin protein PMP22 in Schwann cells: modulation of cell growth. EMBO J 14: 1122-8 
